\title{
SCIENCES NATURELLES.
}

\section{COLLECTION DE FIGURES}

\author{
POUR EN FACILITER L'USAGE.
}

Ces figures sont disposées ici dans l'ordre systématique le plus généralement adopté : celui du règne animal de G. Cuvier, pour la partie Zoologique, et celui des familles naturelles pour la Botanique. Les planches n'ont point été numérotées par le graveur, afin que chacun pùt les placer à son ggré si un ordre différent lui semblait préférable.

\section{PARTIE ZOOLOGIQUE.}

\section{MAMMIFÈRES.}

Quadrumanes.

P1. I. Pithèque Orang-outang; Pithecus satyrus.

Gibbon wouwou; Hylobates leuciscus.

Guenon callitriche; Simia sabloza.

Pl. II. Macaque domestique ou Maimon; Macacus nemestrinus.

Sapajou-Atèle hybride; Ateles hybridus. a. Sa tête vue de profil.

Pl. III. Colobe Guereza ; Colobus Guereza. a. Son crâne. Tarsier spectre; Tarsius spectrum. $b$. Son crâne. c. Ses dents.

\section{Carnassiers.}

PI. IV. Molosse obscur; Molossus obscurus. (La tète en profil.)

Noctilion Lièvre; Noctilio Leporinus. (La tête en profil.)

Phyllostome crénelé; Phyllostoma crenulatum.

Mégaderme trèfle; Megaderma trifolium.

Rhinolophe trident; Rhinolophus tridens. (La tête en face.)

Nyctère de Geoffroy; Nycteris Geoffroyi. (La tête en face.)

PI. V. Hérisson commun; Erynaceus Europaeus. Chrysochlore du Cap; Chrysochloris aurata.

PI. VI. Ours aux grandes lèvres; Ursus labiatus. Arctonix ou Bali-saur à collier; Arctonix collaris.

PI. VII. Coati roux; Viverra nasua.

Blaireau; Meles.

Putois ou Marte Zorille; Putorius Zorilla; Mustela Zorilla.

P1. VIII. Mouffette chinche; Mephitis laticaudata. Mydas Télagon; Mydaus meliceps. Galidie élégante; Galidia elegans.
Pl. IX. Nélogale brune; Melogale fusca. Hyène brune; Hyona fusca.

Pl. X. Civette à bandeau; Viverra fasciata. Paradoxure type; Paradoxurus typus.

Ichneumie albescente; Ichneumia albescens.

PI. XI. Phoque-arctocéphale oursin (Ours marin); Arctocephalus ursinus. 1. Son crâne.

Phoque-platyrhynque lion. Platyrhynchus leoninus. 2. Son crâne.

P1. XII. Kanguroo laineux; Kangurus laniger. a. Le pied postérieur droit. $b$. Portion du pied postẻrieur gauche.

RoNGEURS.

Pl. XIII. Écureuil à ventre doré; Sciurus auriventris. Houtia de Poyei; Capromys Poyeii.

Lemming vulgaire; Lemmus (Georychus, E.) vulgaris.

Pl. XIV. Hamster commun; Mus cricetus.

Gerboise alactaga; Dipus jaculus.

Saccomyde anthrophile; Saccomys anthrophilus.

Castor du Canada; Castor fiber.

PI. XV. Porc-Épic d'Italie; Hystrix cristata.

Éréthizon urson; Hystrix dorsata.

PI. XVI. Chloromyde agouti; Chloromys acuti.

Paca brun; Cologenis subniger.

\section{ÉDENTÉS.}

Pl. XVII. Mégathère. .... ; Megatherium. . . . PI. XVIII. Bradype Aï; Bradypus tridactylus.

Tatou Encoubert; Dasypus minutus.

Chlamiphore tronqué; Chlamiphorus truncatus.

Pangolin de Java; Manis Javanica.

Pachydermes.

Pl. XIX. Deinothérion géant; Dinotherium giganteum. Mastodon très-grand; Mastodon maximus. 
PI. XX. Hippopolame du Cap; Hippopolamus Capensis.

P1. XXI. Cochon Sanglier; Sus scrofa.

Rhinocéros des Indes; Rhinoceros Indicus.

Tapir de l'Inde; Tapirus Indicus.

P1. XXII. Cheval Dziggetai ou Czigithai; Equus hemionus.

\section{RuMinants.}

Pl. XXIII. Cerf de Virginie; Cervus Virginianus. Renne; Cervus tarandus.

PI. XXIV. Girafe; Camelopardalis Girafa.

CÉtacés.

PI. XXV. Dugong; Trichecus Dugong.

Narval; Monodon Narval.

Baleine franche; Balcena mysticetus.

\section{OISEAUX.}

Accipitres ou Rapaces.

PI. XXVI. Catharte alimoche; Vultur percnopterus, L. Faucon-Aigle Pygargue; Falco albicella. Chouetle-Hibou grand-duc; Strix bubo.

\section{Passereaux.}

PI. XXVII. Brève pittoïde; Brachypteracius pittoides. Scythrops présageur; Scythrops Nover-Hollandice. (Cuvier a placé ce genre parmi les Oiseaux grimpeurs.)

Eurylaime corydon; Eurylaimus corydo.

PI. XXVIII. Mainate de Java; Gracula religiosa.

Phonygame de Kéraudren; Phonygama Keraudreni.

Sericule orangé; Sericulus aurantiacus.

Paradisier rouge; Paradisea rubra.

Pl. XXIX. Ménure Lyre; Manura Lyrata.

Pl. XXX. Martinet coiffé; Cypselus comatus.

Guacharo de Caripe; Steatornis Caripensis. a. La mandibule supérieure vue en dessus. $b$. Le pied de grandeur naturelle.

Pl. XXXI. Mésange à huppe jaune; Parus flavo-cristatus.

Gros-bec de Cuba; Fringilla Cubce.

Tisserin fringilloide; Poceus fringilloides.

Pl. XXXII. Bouvreuil commun; Pyrrhula Europara.

Loxie bec-croisé des Pins; Loxia curvirostra.

PI. XXXIII. Picucule bec en coin; Dendrocolaptes cuneatus.

Dicée (Philédon) à plastron rouge; Dicceum erythrogaster.

Tichodrome échelette; Tichodroma phœnicoptera.

PI. XXXIV. Colibri topaze; Trochilus pella.

Colibri à long bec ou corinne; Trochilus longirostris.

PI. XXXV. Guêpier commun; Merops apiaster.

Martin-Pêcheur vintsioide; Alcedo vintsioides.

Martin-Chasseur à coiffe brune; Dacelo fuscicapilla.

PI. XXXVI. Calao rhinocéros; Buceros rhinoceros.

\section{Grimpeurs.}

PI. XXXVII. Aracari grigri; Pteroglossus aracari.

Toucan Toco; Ramphastos Toco.
Pl. XXXVIII. Ara tricolore; Macrocercus tricolor.

Microglosse très-noir; Microglossum aterrimum. a. le bec ouvert, laissant voir la langue.

\section{Gallinacés.}

Argus Luen femelle; Argus pavonius, fem.

PI. XXXIX. Argus Luen mâle; Argus Pavoninus, mas.

PI. XL. Monaul ou Impey resplendissant; Monaulus seu Impeyanus refulgens.

PI. XLI. Mégrapode huppé; Megapodius cristatus. Placé par Cuvier, parmi ses Échassiers.

Turnix à bandeau noir; Hemipodius nigrifrons.

Eudromie élégante; Eudromia elegans.

PI. XLII. Perdrix rouge; Perdix rufa.

Perdix-Gaille nattée; Perdix textilis.

Pl. XLIII. Pigeon-Colombe de Bolivie; Columba Boliviana.

Pigeon-Colombar à gorge pourprée; Columba viridis. Pigeon-Lophyre Goura; Columba coronata.

\section{Échassiers.}

PI. XLIV. Autruche; Struthio camelus.

Casoar ; Casuarius galeatus.

PI. XLV. Agami trompette; Psophia crepitans.

Grue couronnée; Ardea pavonina.

PI. XLVI. Savacou cochlearia; Cancroma cochlearia. Ombrette du Sénégal; Scopus umbretta.

Spatule rose; Platalea ajaja.

Pl. XLVII. Rhynchée du cap de Bonne-Espérance; Rhynchoea Capensis.

Caurale phalénoide; Eurypiga helias.

P1. XLVIII. Leptorhynque à poitrine rousse; Leptorynchus pectoralis, $a$. Son bec vu en dessus. $b$. Le même vu de côté.

Jacana à nuque blanche; Parra albinuca.

\section{PALMiPÈdes.}

Pl. XLIX. Grèbe huppé; Podiceps cristatus. Macareux moine; Mormon fratercula.

Grand Manchot; Aptenodytes patachonica.

Mouette à manteau noir ; Larus marinus.

Pl. L. Pélican blanc; Pelecanus onocrotalus.

Grande Frégate; Tachypetes aquila.

Phaeton à brins rouges; Photon phonicurus.

Pl. LI. Phœnicoptère à manteau couleur de feu; Phønicopterus ignipalliatus.

Canard-0ie à double éperon; Anas Gambensis.

Beau Canard huppé; Anas sponsa.

Pl. LII. Céréopse cendré; Cereopsis Nova-Hollandice. Chionis bec en fourreau; Chionis Nover-Hollandice.

\section{REPTILES.}

\section{Chéloniens.}

Pl. LIII. Émyde peinte; Emys picta. Age adulte. a. Émyde peinte, jeune âge. Emys concinna, Cuv. b. Émyde peinte, vue en dessous.

\section{Sadriens.}

Pl. LIV. Crocodile à deux arêtes; Crocodilus biporcatus. 
a. Tête du Caïman à lunettes; Crocodilus Americanus.

b. Tête du Gavial longirostre; Crocodilus Gangeticus.

PI. LV. Stellion-Doryphore à queue courte; Doryphorus brevicaudatus. Cuv.

Stellion-Fouette-queue réticulé; Uromastix reticulatus.

Tête du Leiolépide tacheté ou à gouttelettes; Leiolepis guttatus. $b$. Forme et disposition des écailles.

Agame-Lyriocéphale margaritacé; Agama margaritacea.

Pl. LVI. Dragon rayé de Java; Draco lineatus.

Caméléon bifide; Chameleo bifidus.

PI. LVII. Basilic porte-crête d'Amboine ; Basilicus A mboinensis.

PI. LVIII. Phyllure de Milius; Phyllurus Milii.

Phyllure de Cuvier; Phyllurus Cuvierii.

Pl. LIX. Scinque bleu; Scincus cœlestinus.

Seps strié; Zygnis striata. a. La tête vue en dessus. $b$. Partie du dessous du corps, $c$. Patte postérieure. d. Patte antérieure.

Triton rayé; Triton vittalus.

\section{Ophidiens.}

Pl. LX. Erpéton tentaculé; Erpeton tentaculatus.

PI. LXI. Crotale à losanges; C'rotalus rhombifer.

Naïa à lunettes; Naia lutescens.

\section{Batraciens.}

PI. LXII. Grenouille verte; Rana esculenta. Rainette à tapires; Hyla tinctoria.

Pipa commun; Pipa vulgaris.

Crapaud accoucheur; Bufo obtetricans.

Éphippifère de Spix; Ephippifer Spixii.

\section{POISSONS.}

\section{ACANTHOPTÉRYGIENS.}

Pl. LXIII. Grammiste oriental; Grammistes orientalis. Diploprion à deux bandes; Diploprion bifasciatum . Serran Mérou; Serranus hexagonatus. Mésoprion Aya ; Lutjanus Aya.

Pl. LXIV. Pomatome télescope; Pomatomus telescopus. Mulle-Surmulet rouget; Mullus ruber.

Pl. LXV. Ptéroide volant; Pterois volitans. Gastérostée épinochette; Gasterosteus pungitius. Chevalier américain; Eques americanus.

Pl. LXVI. Pristipome pique; Pristipomus hastatus. Holocentre Sogo; Holocentrus Sogo.

Pl. LXVII. Pentapode bandelette; Pentapodus vitta.

PI. LXVIII. Picarel Raillard; Smaris manritianus.

Pl. LXIX. Espadon commun; Xiphias gladius. 2. Sa mandibule supérieure ou glaive, vue en dessus; อ. La même vue de côté; 4 . Coupe du corps. Sériale de Duméril ; Seriala Dumerilii.

\section{Pharyngiens Labyrinthiformes.}

Pl. LXX. 1. Lophie Baudroie; Lophius piscatorius. $a$. Le même vu en dessus.

2. Batracoïde de Surinam; Batracoides Surinamensis.

5. Chironecte des côtes; Antennarius nesogallicus. a. Le mème vu en dessus.
Nota. A la légende de cette planche, le graveur a fait erronément une transposition des chiffres 2 et 5.

Pl. LXXI. Gomphose Lacépède; Gomphosus tricolor.

PI. LXXII. Centrisque Bécasse de mer; Centriscus Scolopax. $a$. Coupe du corps.

Amphisèle cuirassé; Centriscus scutatus.a. Coupe du corps.

\section{MALACOPTÉRYGIENS.}

\section{Abdominaux.}

Pl. LXXIII. Mormyre oxyrhynque; Mormyrus oxyrhynchus.

Schilbé Barbeau; Schilbœus (Silurus) Mystus.

PI. LXXIV. Hétérobranche Harmont; Heterobranchus anguillaris.

Malaptérure électrique; Malapterurus electricus.

Subrachiens.

PI. LXXV. Cycloptère Lompe; Cyclopterus Lumpus. a. Coupe du corps.

Apodes.

PI. LXXVI. Ophisure Serpent de mer; Murcena Serpens. Murène Zebre; Murana Zebra. a. Coupe du corps.

Sphagébranche aveuggle; Murcena caca. $b$. La tête vue en dessous.

\section{LOPHOBRANCHES.}

Pl. LXXVII. Syngnathe vert; Syngnathus viridis. $a$. Sa tête de grandeur naturelle.

Hippocampe brévirostre; Hippocampus brevirostris. Pégase Dragon ; Pegasus Draco. $b$. Sa tête vue en dessus.

\section{Chondroptérygiens.}

PI. LXXVIII. Callorynque antarctique; Callorynchus antarcticus.

Squale centrine; Squalus centrina. $a$. La tête vue en dessous.

Leiche Laborde; Scymnus mauritianus. (PI. LXVIII.)

PI. LXXIX. Rhinobate lisse; Rhinobatus leevis, $a$. Ses mâchoires.

Torpille Narke; Torpedo Narke.

Céphaloptère Giorna; Cephaloptera Giorna.

Trygon de Halgan; Trygon Halgani.

Pl. LXXX. Trygon torpédine; Trygon torpedinus.

Pétromizon Lamproie; Petromizon Marinus. a. Ouverture de la bouche.

\section{MOLLUSOUES.}

\section{Céphalopodes.}

PI. LXXXI. 1. Poulpe de Cuvier; Octopus Cuvieri.

2. Argonaute Argo; Argonauta Argo. a. Sa coquille.

3. Sèche commune; Sepia officinalis, a. Son sépiostaire.

4. Calmar de Brongniart; Loligo Brongniartii.

5. Nautile pompile; Nautilus pompilius.

6. Spirule australe; spirula australis. a. Sa coquille. $b$. $c$. Cloisons de la coquille, vues isolément et assemblẻes. 
7. Extrémité d'un grand bras de l'Onychoteuthis angulata. $a$. Rudiment testacé interne en forme d'épée.

8. Extrémité d'un bras de l'Eledone moschatus.

PI. LXXXII. 1. Bẻlemnite d'Osterfield; Belemnites Oster. fieldeus.

2. Conilite anguliforme; Conilites anguliformis.

๊. Ammonite de Bayeux; Ammonites Bajocce.

4. Orbiculine numismale; Orbiculina numismalis.

5. Scaphite oblique; Scaphites obliquus.

6. Baculite vertébrale; Baculites vertebralis.

7. Hamite cylindrique; Hamites cylindrica.

8. Amplexe coralloide; Amplexus coralloides.

9. Turrilite turbinée; Turrilites turbinatus.

Gastéropodes.

Pl. LXXXIII. 1. Aggathine Zèbre; Achatina Zebra.

2. Agathine gland; Achatina glans.

5. Agathine columnaire; Achatina columnaris.

4. Maillot bossu; Pupa gibbosa.

5. Hélice conoïde; Helix conoides.

6. Hélice naticoide; Helix naticoides.

7. Hélice Planorbe; Helix Planorba.

8. Hélice Péson; Helix Algira.

Pl. LXXXIV. 1. Télescope vulgaire; Cerithium telescopium.

2. Toupie pagode; Trochus pagodus.

5. Cadran perspective; Solarium perspectivum.

4. Sabot rugueux; Turbo rugosus.

5. Scalaire précieuse; Scalaria preciosa.

6. Ampullaire de la Guiane; Ampullaria Guianensis.

7. Natice glaucine; Natica glaucina. L'animal développé.

PI. LXXXV. Hipponice corne d'abondance; Hipponix cornupia.

2. La même coquille vue intérieurement.

3. Crépidule subspirée; Crepidula subspirea.

4. Navicelle elliptique; Navicella elliptica.

5. Cône impérial; Conus imperialis.

6. Porcelaine églantine; Cyprcea eglantina.

7. Ovule des Moluques; Ovula Moluccana.

8. Navelte valve; Bulla valva.

9. Ultime gुibbeux; Ultimus gibbosus.

10. Tarière subulée; Terebellum subulatum.

Pl. LXXXVI. 1. Olive polpasta; Oliva polpasta.

2. Volute de Neptune; Voluta Neptuni. Animal développé.

3. Volute thiarelle; Voluta thiarella.

4. Marginelle neigeuse; Marginella nivosa.

5. La même coquille avec l'animal.

6. Mitre de Lamarck; Mitra Lamarcki.

7. Cancellaire impériale ; Cancellaria imperialis.

Pl. LXXXVII. 1. Casque Bezoar; Cassis alguca. L'anımal développé.

2. Harpe ventrue; Harpa ventricosa. L'animal développé.

5. Buccin écaille ; Buccinum testudineum. L'animal développé.

Pl. LXXXVIII. 1. Cérite chenille; Cerithium aluco.

2. Rocher forte-épine; Murex crassispina.

5. Apolle Gyrin; Murex Gyrinus.

4. Struthiolaire noduleuse; Struthiolaria nodulosa.
5. Fasciolaire de Tarente; Fasciolaria Tarentina. L'animal développé.

6. Ptérocère cornu; Pterocera cornuta.

7. Rostellaire pied de Pélican; Rostellaria pes-Pelecani. L'animal développé.

Pl. LXXXIX. 1. Siliquaire muriquée ; Sitiquaria muricata.

2. a. Haliotide canaliculée; Halyotis canaliculata.

b. La coquille et l'animal développé.

5. a. Fissurelle annelée; Fissurella annulata.

b. La coquille et l'animal développé.

4. a. Patelle pyramidée; Patella pyramidata. La coquille est vue en dessous, avec l'animal qu'elle loge. b. L'animal isolé.

5. a. Oscabrion écailleux; Chiton squamosus.

$b$. La même coquille vue en dessous et avec l'animal.

ACÉPHALES.

Pl. XC. 1. Huître crête de Coq ; Ostrea crista-galli.

2. Spondyle américain; Spondylus americanus.

5. Marteau vulgaire; Malleus vulgaris.

4. Avicule Hironde; Avicula Hirundo.

5. Pinne hérissée; Pinna nobilis.

7. Tridacne gigantesque; Chama gigas.

8. La même coquille vue du côté de la charnière.

9. Hippope maculé; Hippopus maculatus.

PI. XCI. 1. a. Donace bec de flute; Donax scortum.

b. La même coquille vue du côté de la charnière.

2. $a$. Donace des Canards; Donax anatinum.

$b$. La même coquille vue du côté de la charnière.

5. a. Capse du Brésil ; Capsa Brasiliensis.

b. La même coquille vue du côté de la charnière.

4. Telline soleil-levant; Tellina radiata.

Pl. XCII. 1. Cyclade cornée; Cyclas cornea.

2. Cyclade de Ceylan; Cyclas Ceylanica.

5. Galathée à rayons; Galathoa radiata.

4. Crassatelle sillonnée; Crassatella sulcata.

5. Mactre Lisor; Mactra Stultorum.

6. Onguline transverse; Ungulina transversa.

7. Érycine cardioide; Erycina cardioides.

BRACHOPODES.

Pl. XCIII. 1. Lingule anatine; Lingula anatina.

2. Térébratule dorsale; T'erebratula dorsalis.

5. Térébratule ailée; Terebratula alata.

4. Térébratule canalifère; Terebratula canalifera.

5. Orbicule de Norwége; Orbicula Norwegica.

6. Cranie en masque; Crania personata.

\section{Cirrhopodes.}

7. Pollicipède groupé; Pollicipeda cornucopia.

8. Cineras à bandes; Cineras vittata.

9. Balane tulipe; Balanus tulipa.

10. Coronule diadème; Coronula diadema.

11. Tubicinelle des Baleines; Tuticinella Balcenarum.

\section{ANIMAUX ARTICULÉS.}

\section{ANNÉLIDES.}

PI. XCIV. 1. Phyllodoce de Paretto; Phyllodoce Paretlo. $a$. La tête grossie.

$b$. Un pied avec sa lame fortement grossis. 
2. Aphrodite hérissée; Aphrodita aculeata.

3. Polynoé épineuse; Polynoe spinosa.

4. Pontopdelle épineuse; Pontopdella spinosa.

5. Clepsine bioculée; Clepsine bioculata.

\section{ANIMAUX RAYONNÉS OU ZOOPHYTES.}

\section{ÉchinOdermes,}

Pl. XCV. 1. Astérie vulgaire; Asterias vulgaris.

2. Crinoïde Lys-de-Mer; Encrinus liliiformis.

5. Oursin comestible; Echinus esculentus.

4. Spatangue velu; Spatangus villosus.

5. Holothurie comestible; Holothuria edulis.

6. Porpite géante; Porpita gigantea.

PI. XCVI. 1. Lucernaire auricule; Lucernaria quadricornis.

2. Tubipore pourpre; Tubipora purpurea.

3. Flustre foliacé; Flustra foliacea.

4. Flabellaire raquette; Flabellaria pinnata.

5. Udotée flabellée; Udolea flabellata.

6. Réceptaculite de Neptune; Receptaculites Neptuni.

\section{Polypes.}

Pl. XCVII. $a$. Actinie verte; Aclinia viridis.

b. La même vue horizontalement.

$c . d$. La même dans son jeune âge.

e.f.g. Tentacules isolés et variés.

PI. XCVIII. 1. Oculine rose; Oculina rosa.

$a$. Une portion grossie, pour montrer l'orifice des branches latérales.

2. Millépore corne d'Élan; Millepora alcicornis.

5. Sériatopore piquant; Seriatopora subulata. a. Portion grossie.

\section{CRUSTACÉS.}

\section{DÉcapodis.}

Pl. XCIX. 1. Mursie mains en crète; Mursia cristata.

2. Gélasime de Tanger; Gelasima Tangeri.

3. Eurynome rugueuse; Eurynoma aspera.

4. Sténorhynque faucheur; Stenorhynchus phalangium.

Pl. C. 1. Thie luisante; Thia polita.

2. Coryste denté; Corystes personatus.

5. Leucosie craniolaire; Leucosia craniolaris.

4. Ethra raboteux; Ethra rugosa.

5. Ranine dentée; Ranina serrata.

6. Hippe émérite; Hippa emerita.

7. Rémipède Tortue; Remipes Testudinarius.

8. Galatée élancée; Galatea strigosa.

Pl. CI. Parthénope horrible; Parthenope horrida.

2. Mithrax à bords épineux; Mithrax spinocinctus.

5. Micippe phylire; Micippa phylira.

4. Camposcie retuja; Camposcia retuja.

Pl. CII. Dromie très-velue; Dromia Rumphii.

Dynamène hispide; Dynamene hispida.

Pl. CIII. Pagure sanguinolent; Pagurus sanguinolentus.

Pagure moucheté; Pagurus guttalus.

Pl. CIV. Langouste bordée; Paleniurus marginatus.
Pl. CV. Callianasse souterraine; Callianassa subterranea.

Cranyon vulgaire; Cranyon vulgaris.

\section{Stomapodes.}

Squille queue rude; Squilla scabricauda. Anilocre du Cap ; Anilocra Capensis.

Ega entaillée; Ega emarginata.

Branchiopodes.

Lépidure prolongé; Lepidurus productus.

PCECILOPODES.

Pandure bicolore; Pandurus bicolor.

PI. CVI. Limule polyphème; Limulus polyphemus.

$a a$. Appendices en forme de pinces insérées sur un labre. $b . c . d$. e. f. $g$. Longues paltes terminées en pince dont les hanches épineuses servent de mâchoires.

$h$. Dernière longue patte pourvue d'une division $h^{\prime}$ en languette et de quatre digitations mobiles à la base de la pince $h^{\prime \prime}$.

$i$. Patte de la première paire.

$k$. Lèvre inférieure.

l. Pharynx.

$m$. $n$. Feuillets recouvrant les branchies.

\section{ARACHNIDES.}

\section{Pulmonatres.}

Pl. CVII. 1. Mygale fasciée; Mygale fasciata.

2. Cténize chasseur ;

3. Épeire militaire; Epeira militaris.

4. Lycose Xyline; Lycosa Xylina.

5. Érèse rouge; Eresus cinnabarinus.

Pl. CVIII. Phryne lunulé ; Phrynus lunatus. Scorpion héros; Scorpio heros.

\section{INSECTES.}

\section{Mrriapodes.}

Pl. CIX. 1. Iule très-grand; Tulus maximus.

2. Polydème granuleux; Polydesmus granulosus.

3. Pollyxène lagure; Pollyxenus lagurus.

4. Scolopendre mordante; Scolopendra morsitans.

5. Scutigère aranéoïde; Scutigera araneoides.

\section{Thysanoures.}

6. Podure plombée; Podura plumbœa. Grossi.

\section{Parasites.}

7. Philoptère de l'Albatros; Philoptera Diomedece. Grossi.

\section{Suceurs.}

8. Puce irritante; Pulex irritans, Grossi.

Coléoptères.

Pl. CX. 1. Anthie à quatre gouttes; Anthia quadriguttata.

3. Tachype doré; Tachypus auratus.

2. Cychre à bec; Cychrus rostralus. 
4. Calosome sycophante; Calosoma sycophanta.

5. Brachyne pétard; Brachinus crepitans.

6. Bembidion à quatre gouttes; Bembidium quadriguttatum.

PI. CXI. 1. Helluo velu; Helluo hirtus.

2. Agre fort petit; Agra parvula.

5. Agre brentoide; Agra brentoides.

4. Cordiste acuminé; Cordistes acuminatus.

5. Cordiste tacheté; Cordistes maculatus.

6. Casnonie cyanocéphale; Casnonia cyanocephala.

7. Colleide convenable; Colleida decora.

8. Catascope à bandes; Catascopus fascialis.

Pl. CXII. 1. Bupreste de Buquet; Buprestis Buqueti.

2. Polybotride Crésus; Polybotris Cresus.

5. Hétérosterne buprestoïde; Helerosternus buprestoides.

4. Taupin acuminé; Elater acuminatus.

5. Taupin linée; Elater lineatus.

Pl. CXIII. 1. Rhipicère bleu; Rhipicera cyanea.

2. Lyque très-large; Lycus latissimus.

3. Malachie ruficolle; Malachius ruficollis.

4. Clairon d'Olivier; Clerus Olivierii.

5. Ptilin serraticorne; Plilinus serraticornis.

6. Atractocère molorchoïde; Atractocerus molorchoides.

7. Mastige brun; Mastigus brunneus.

8. Escarbot en rein; Hisler reniformis.

9. Nécrophore maritime; Necrophorus maritimus.

10. Scaphidie nigripède; Scaphidium nigripes.

11. Thymale marginicolle; Thymalus marginicollis.

12. Mégatome ondé; Megatoma undatum.

13. Hydrophile spinipenne; Hydrophilus marginipennis.

P1. CXIV. 1. Aphodie fimetaire; Aphodius fimetarius.

2. Ateuche sacré; Ateuchus sacer.

5. Bousier lunaire; Copris lunaris.

4. Cétoine métallique; Cetonia metallica.

Pl. CXV. 1. Goliath cacique; Goliath cacicus.

2. Geotrupe phalangiste; Geotrupes tiphæus.

כ. Hanneton foulon; Melolontha fullo.

4. Oryctès nasicorne; Oryctes nasicornis.

5. Trichie noble; Trichius nobilis.

6. Trox hérissé: Trox hispidus.

Pl. CXVI. Sphénognathe prionoide; Sphenognathus prionoides.

2. Pholidote de Humboldt; Pholidotus Humboldtii.

5. Passale de Gory; Passalus Goryi.

4. Calognathe de Chevrolat; Calognathus Chevrolati.

5. Steire à côtes; Steira costata.

6. Molure de Pierret; Moluris Pierreti.

7. Opâtre gris ; Opatrum griseum.

8. Léiode cannelé; Leiodes striata.

9. Sphénisque peint; Spheniscus pictus.

10. Hallomène huméral; Hallomenus humeralis.

11. Dytile Jisse; Dytilus lovigatus.

12. Myodite américain; Myodites americana.

15. Méloé d'Olivier; Meloe Olivieri.

Pl. CXVII. 1. Attelabe somptueux; Attelabus somptuosus.

2. Aterpe pipa; Aterpus pipa.

3. Calodrome de Melly; Calodroma Mellyi.
4. Eutrachèle de Temminck; Eutracheles Temminckii.

5. Géonème à bandes; Geonemus vittatus.

6. Chole à bandes jaunes; Cholus flavicinctus.

7. Trigonotarse calandroide; Trigonotarsis calandroides.

8. Phloïotribe de l'Olivier; Phloiotribus olea.

9. Tessérocère remarquable; Tesserocerus insignis.

10. Pausse cornu; Paussus cornutus.

11. Bostriche de Dufour; Bostrichus Dufourii.

12. Synchite ondé; Synchita undata.

13. Cucuje à pattes jaunes; Cucujus flavipes.

PI. CXVIII. Tricténotome de Children; Trictenotoma Children.

Hamatichère sutural; Hamaticherus suturalis.

PI. CXIX. 1. Rosalie des Alpes; Rosalia Alpina.

2. Onychocère Scorpion; Onychocerus Scorpio.

5. Lamie ponctateur; Lamia punctator.

4. Phœdine tricolore; Phœdinus tricolor.

5. Saperde carcharias; Saperda carcharias.

6. Vespère grec; Vesperus grcecus.

7. Sagre de Boisduval; Sagra Boisduvalii.

8. Hispe de Fabricius; Hispa Fabricii.

9. Molacagastre de Passerini; Malacogaster Passerinii.

10. Chrysomèle humérale; Chrysomela humeralis.

11. Trochalanote brun-bai; Trochalanota badia.

12. Galéruque smaragdine; Galeruca smaragdina.

13. Octogonate de Banon; Octogonatus Banoni.

Pl. CXX. 1. Dasycère sillonné; Dasycerus sulcatus.

a. L'antenne grossie.

b. Un tarse grossi.

2. Endomyque écarlate; Endomychus coccineus.

5. Eumorphe de Sumatra; Eumorphus Sumatrensis.

a. L'insecte vu de profil.

b. Antenne grossie.

c. Jambe antérieure avee son tarse grossis.

4. Scymne à écusson; Scymnus scutatus.

5. Coccinelle à dix-neuf points; Coccinella 19-punctata.

Pl. CXXI. 1. Coccinelle ocellée; Coccinella ocellata.

2. Coccinelle à plaie; Coccinella plagata .

a. L'insecte vu de profil.

5. Pselaphe hématique; Pselaphus homatichus.

a. Antenne grossie.

b. Patte postérieure grossie.

c. Tarse grossi très-fortement.

4. Chennie bituberculé; Chennium bituberculatum. a. La tête grossie.

b. Tarse très-ģrossi.

5. Claviger longicorne; Claviger longicornis. a. L'antenne très -grossie.

b. Antenne très-grossie du Clavigger à fossettes; Claviger foveolatus.

\section{Orthoptères.}

PI. CXXII. 1. Forficule crocipenne; Forficule crocipennis.

2. Blatte peinte; Blatta picta.

3. Mante chlorophée; Mantis chlorophara.

4. Bacterie scabreuse; Bacteria scabra. 
5. Phyllie feuille sèche; Phyllium siccifolium.

Pl. CXXIII. 1. Gryllon de Serville; Gryllus Servillii.

2. Courtillière tridactyle; Gryllo-Talpa tridactyla.

5. Sauterelle érythrosome; Locusta erythrosoma.

4. Truxale nasu; Truxalis nasuta.

5. Criquet voyageur; Acridium viator.

\section{HÉMIPTÈRES.}

Pl. CXXIV. 1. Scutellère riche; Scutellera dives.

2. Phida cassidoide; Phida cassidoides.

3. Anisoscèle alipède; Anisoscelis alipes.

4. Fulgore porte-chandelle; Fulgora candellaria.

5. Fulgore bigarrée; Fulgora variegata.

6. Aphène discolore; Aphcena discolor.

7. Membrace foliée; Membracis foliata.

8. Céphalèle enfumé; Cephalelus brunneus.

Pl. CXXV. 1. Aleyrode de l'Éclaire; Aleyrodes Chelidonii.

a. La tête vue en dessors.

2. Cochenille du Nopal; Coccus Cacti. Mâle.

a. Cochenille du Nopal femelle, vu en dessus.

$b$. Le même insecte vu en dessous.

c. Une palte très-grossie.

3. Puceron du Rosier; Aphis Rosae.

a. Sa larve.

4. Kermès oblong du pêcher, mâle; Chermes oblongus persica.

$a$. Le même insecte vu les ailes étendues.

5. Psylle du Jone; Psylla Junci.

NÉVROPTẺres.

PI. CXXVI. 1. Libellule déprimée; Libellula depressa.

2. Éphémère vulgaire; Ephemera vulgaris.

5. Panorbe commune; Panorpa communis.

4. Némoptère à balanciers; Nemoptera halterata.

5. Fourmilion de Percheron; Myrmileo Percheronii.

6. Ascalaphe italien; Ascalaphus italicus.

7. Hémerobe Chrisope; Hemerobius Chrysops.

8. Raphidie Serpent; Raphidia Ophiopsis.

9. Psoque à deux points; Psocus bipunctatus.

10. Perle à deux queues; Perla bicaudata.

HYMÉNOPTÈRES,

Pl. CXXVII. 1. 2. 3. 4. 5. 6. Analyse et définition des diverses parties qui constituent l'aile antérieure des Hyménoptères.

1. $a$. Extrémité de l'aile.

b. Base de l'aile; point d'insertion au thorax.

c. Angle postérieur de l'aile; il est situé entre le bord postérieur et le bord interne.

d. Bord externe; il s'étend depuis la base de l'aile jusqu'à son extrémité.

$e$. Bord postérieur ; il s'étend depuis l'angle postérieur jusqu'au bout de l'aile.

$f$. Bord interne; il s'étend depuis l'angle postérieur jusqu'à la base de l'aile.

g. Disque; c'est tonte la partie comprise entre les bords.

2. a. Rayon; c'est la première nervure du bord externe de l'aile; il s'étend depuis la base jusqu'an point. b. Os du coude, seconde nervure du bord externe de l'aile; il s'étend depuis la base jusqu'au point de l'aile; il est séparé du rayon par une membrane intermédiaire.

c. Point ou carpe; il est situé à la terminaison des os de l'avant-bras; c'est entre ces deux parties que se trouve l'articulation.

d. Cellules radiales.

$e$, Cellules cubitales.

$f$. Nervures récurrentes; elles remontent des nervures du disque de l'aile pour s'insérer dans les cellules cubitales.

g. Nervures brachiales; elles naissènt du thorax, et s'unissent avec les nervures de l'avant-bras.

5. $a$. Cellule radiale appendicée.

b. Cellule cubitale pétiolée.

$i$. Cellules discoïdales.

4. $a$. Le carpe.

$b$. Cellule incomplète.

5. $a$. Point de l'aile.

$e$. Nervures récurrentes.

$h . h . h$. Nervures humérales.

6. Nervures fortement grossies, pour montrer les bulles qui semblent interrompre la continuité de ces nervures.

7. Aptérogyne d'Olivier; Apterogyna Olivieri.

8. Aptérogyne unicolore; Apterogyna unicolor.

9. Andrène jolie; Andrena pulchella.

10. Abeille mellifique; Apis mellifica.

Pl. CXXVIII. 1. Hylotome du Rosier; Hylotoma Rosce.

2. Urocère géant; Urocerus gigas.

5. Pélécine de Duponchel; Pelecinus Duponchelii.

4. Osprynchote du Cap; Osprynchotus Capensis.

5. Cérocéphale cornigère; Cerocephalus corniger.

6 Scolie des jardins; Scolia horlorum.

7. Pepside marginé; Pepsis marginata.

8. Crabron à crible; Crabro cribrarius.

9. Mégachile soyeux; Megachile sericea.

10. Melissode de Fonscolombe; Melissoda Fonscolombi.

11. Acanthope splendide; Acanthopus splendidus.

\section{LÉPIDOPTÈRES}

Pl. CXXIX. Uranie Prométhée; Urania Prometheus.

Pl. CXXX. 1. Sésie fréloniforme; Sesia crabroniformis.

2. Procris du Statice; Procris staticis.

3. Syntomide de Kuhlwein; Syntomis Kuhlweinii.

4. Zygène de l'Esparcette; Zygæna onobrichidis.

5. Macroglosse bombyliforme; Macroglossum bombyliforme.

6. Thyride d'Abbot; Thyris Abbotii.

7. Ptérogon de l'Onagre; Pterogon OEnotherce.

8. Deilephile du Nérion; Deilephila Nerii.

9. Smérynthe oculée; Smerynthus ocellatus.

Pl. CXXXI. 1. Bombyce matrone; Arctia matronula.

2. Bombyce hérisson; Arctia casa.

5. Bombyce moucheté; Arctia purpurea.

PI. CXXXII. 1. Noctuelle du Frène; Noctua fraxini.

2. Noctuelle fiancée; Noctua sponsa.

3. Noctuelle mariée; Noctua nupta. 
DiptèrRs.

Pl. CXXXIII. 1. Cousin commun; Culex pipiens. Le mâle grossi.

$a$. Le même insecte à l'état de larve.

$b$-c. Le même à l'état de nymphe vue naturellement et étendue.

2. Bombyle peint; Bombylus pictus.

3. Hippobosque des Chevaux; Hippobosca Equina. a. Les mandibules.

4. Conops à pattes jaunes; Conops flavipes.

a. La bouche vue de profil.

5. Myope noir; Myopus niger.

$a$. La bouche vue de profil.

PI. CXXXIV. 1. Anthropolite de la Guadeloupe.

2. Protée fossile.

5. Squelette d'une tête de Salamandre.

\section{PARTIE BOTANIQUE.}

\section{CRYPTOGAMIE.}

\section{Arthrodiées.}

PI. CXXXY et CXXXVI. Fragillaires. 1. $a$. b. Diatome vulgaire; Diatoma vulgaris.

c. Diatome danois; Diatoma danica.

2. Achnanthe adnée; Achnanthes adnata,

3. a. Nématoplate argentée; Nematoplata argentala.

b. Nématoplate capucine; Nematoplata capucina.

Oscillariées. 4. Dilwynelle serpentine; Dilwynella serpentina.

5. b. Oscillaire urbique; Oscillaria urbica.

d. Oscillaire ténioïde; Oscillaria tonioidea.

h. Oscillaire élégant; Oscillaria elegans.

6. a-c. Vaginaire terrestre; Vaginaria terrestris.

7. a-c. Anabaine fausse-Oscillaire; Anabaina Oscillarioides.

d. Anabaine membranine; Anabaina membranina.

Conjugées. 8. a. Leda moniline; Leda monilina.

$b \sim c$. Leda des landes; Leda sabuletorum.

9. $a-d$. Tendaridée Pollux; Tendaridea Pollux. $e-g$. Tendaridée Castor; Tendaridea Castor.

10. Salmacide brillante; Salmacis nitida.

11. a-c. Zignème bulleuse; Zignema bullosa.

Zoocarpées. 12. Anthophyse dichotome; Anthophysis dichotoma.

a. Filament de Salmacide grossie d'oủ s'élève l'anthophyse également grossie.

$b$-c. Groupes d'anthophyses à l'état naturel.

d. Anthophyses isolés.

13. a. Tirésias en collier; Tiresias monoliformis.

b-l. Tirésias crépue; Tiresias crispa.

14. $a \cdot d$. Cadmus soyeuse; Cadmus sericea.

\section{LICHFNs.}

Pl. CXXXVII. 1. Gassicourlie écarlate ; Gassicurtia coccinea.

2. Calycier chrysocéphale; Calycium chrysocephalum.

5. Sarcographe des Quinquinas; Sarcographa Cinchonarum.

4. Verrucaire de Gaudichaud; Verrucaria Gaudichaldii.

5. Collema azuré; Collema azureum.

6. Scyphophore didyme; Scyphophorus didymus.
7. Parmélie couronnée; Parmelia coronata.

8. Plectocarpe de Delise; Plectocarpum Delisei.

9. Solorine des Quinquinas; Solorina Cinchonarum. 10. Cétraire du Geniévrier; Cetraria Juniperina.

Champignons.

Pl. CXXXVIII. 1. Lycogale ponctuée; Lycogala punctatum.

a. Sporules et filaments grossis.

2. a. Cionier xanthope; Cionium xanthopus; de grandeur naturelle.

b. La plante grossie.

c. Coupe d'un péridion.

3. a. Cratérier pyriforme; Craterium pyriforme; de grandeur naturelle.

$b$. La plante grossie.

c. Sporules et filaments grossis.

4. Arcyrie incarnée; Arcyria incarnata; de grandeur naturelle.

b. La même grossie à diverses époques du développement.

5. Stémonitide leucopode; Stemonitis leucopodia; de grandeur naturelle.

b. La même grossie à diverses époques du développement.

b. Stémonitite ovale; Slemonites ovata; de grandeur naturelle.

b. La même grossie.

c. Le péridion après la dispersion des sporules.

d. Sporules à diverses époques du développement.

Pl. CXXXIX. 1. Agaric orangé; Agaricus aurantiacus.

2. Pezize bleue; Peziza cœrulea.

3. Trémelle oreille de Judas; Tremella auricula.

4. Clathre frisé; Clathrus cirrhatus.

5. Phallus orangé; Phallus impudicus.

6. Lycoperdon ciselé; Lycoperdon colatum.

7. Lycoperdon gigantesque; Lycoperdon giganteum .

8. Truffe comestible; Tuber cibarium.

9. La même plante coupée par le milieu.

Hépathoues, Mousses, Fougères, etc.

P. CXL. 1. Cyathéa en arbre; Cyathea arborea.

2. Polypode piloselloide; Polypodium piloselloides.

5. Pylopode à feuilles épaisses; Pylopodium crassifolium. 
4. Lycopode phlegmaire; Lycopodium phlegmaria.

5. Lycopode penché; Lycopodium cernuum.

6. Prêle fluviatile; Equisetum fluviatile.

7. Sphaignes à larges feuilles; Sphagnum latifolium.

PI. CXLI. Acrostic écussonné; Acrosticum alcicorne.

$a$. Portion d'une feuille grossie.

$b$. Poils cloisonnés, disposés en étoile à la base des capsules et très-fortement grossis.

c. Une capsule très-grossie.

d. La même vue de côté.

$e$. La même déchirée et lançant, par élasticité, les corps reproducteurs.

\section{PHANÉROGAMIE.}

\section{DicotyLÉdONES.}

PI. CXLII. 1. Platane d'Orient; Platanus Orientalis.

2. Pin maritime; Pinus maritima.

5. Digitale pourprée; Digitalis purpurea.

4. Cierge opuntia; Cactus opuntia.

5. Dionée Attrape-Mouche; Dioncea Muscipula.

6. Dodécathée de Virginie; Dodecatheon Meadia.

7. Mélocacte déprimé; Melocaclus depressus.

PI. CXLIII. Clématite violette; Clematis corulea.

Pl. CXL1V. Magnolie de Soulange; Magnolia Soulangiana.

PI. CXLV. Hydropeltide purpurine; Hydropeltis purpurea.

1. Étamine.

2. Pistil.

3. Le même coupé, afin de montrer la position des ovules.

4. Fruits présentant différents développements.

5 . Un fruit coupé, montrant en $a$ un ovule avorté.

6. Graine dont on a enlevé la moitié du tégument; en $a$ se voit l'embryon.

7. Amande nue.

8. Portion d'une amande montrant en $a$ l'embryon coupé.

9. Embryon isolé.

10. Embryon coupé, $a$. Graine cotylée. $b$. Gemmule.

Pl. CXLVI. Sarracénie rouge; Sarracenia rubra.

1. Une étamine.

2. Le pistil.

PI. CXLVII. Giroflée voilier; Cheiranthus Cheiri.

a. La silique ouverte par la base, montrant la position des graines sur la cloison.

PI. CXLVIII. Rocouier cultivé; Bixa orellana.

1. Une fleur vue en dessous.

2. Un pétale isolé.

3. Une étamine.

4. Le pistil, $a$. Glandes caliculaires.

5. Fruit s'ouvrant pour la dispersion des graines.

6. Graine munie de son pédicule ou cordon ombilical.

7. Graine coupée verticalement pour montrer la position de l'embryon au milieu de l'endosperme.

8. Embryon.

9. Graine en germination. $a$. Ligne médiane.

Pl. CXLIX. Sollye hétérophylle; Sollya heterophylla.
Pl. CL. Cotonnier à trois pointes; Gossipium Iricuspidatum.

1. Corolle staminifère ouverte.

2. Pistil accompagné en $a$ de trois feuilles involucrales et en $b$ du calice.

5. Péricarpe coupé transversalement pour montrer les trois loges et le point d'attache des graines dans l'angle des loges.

4. Une graine chargée de son duvet.

5. Graine coupée transversalement.

6. Embryon isolé.

Pl. CLI. Dombeye à feuilles réfléchies; Dombeya reflexa.

1. Le calice étalé.

2. Le pistil.

3. Une étamine.

4. Le pistil et les étamines, étalés et grossis.

5. L'ovaire coupé verticalement pour montrer les loges et la position des ovules.

Pl. CLII. Camellie du Japon; Camellia Japonica.

2. Le pistil isolé.

3. Le calice.

4. La variélé dite Pomponia, dans laquelle la plupart des étamines ont pris un développement pétaliforme.

Pl. CLIII. Mamei ou Mammei d'Amérique; Mammea Americana.

1. Une étamine grossie.

2. Le pistil.

3. Une graine.

4. La même coupée transversalement.

Pl. CLIV. Capucine tubéreuse; Tropcoolum tuberosum.

1. La fleur coupée verticalement.

2. Le pistil grossi.

Pl. CLV. Érythrochiton du Brésil ; Erythrochiton Brasiliensis.

1. Le pistil.

2. L'ovaire coupé verticalement, montrant la position des ovules.

3. La plante entière, tracée en petit.

PI. CLVI. Clitore de Virginie; Clitoria Virginiana.

Pl. CLVII. Liane à réglisse; Abrus priscatorius.

1. Fleur isolée.

2. Les pétales.

3. La carène.

4. Calice, étamines et pistil.

5. Calice et pistil.

6. Pistil.

7. Le fruil.

8. Une graine montrant en $a$ le micropyle.

9. La même coupée transversalement.

10. Embryon, a. Chalaze.

11. Embryon vu de côté.

Pl. CLVIII. Ériobotrye du Japon; Eriobotrya Japonica. a. Le fruit.

PI. CLIX. Fuchsie conique; Fuchsia conica.

PI. CLX. Godetie rubiconde; Godetia rubicunda.

Pl. CLX1. Rhexie superbe; Rhexia princeps.

Pl. CLXII. Metrosideros à feuilles glauques; Metrosideros glauca.

1. Une fleur de grandeur naturelle.

2. La même coupée verticalement. 
5. Un pétale isolé.

4. Étamines soudées par leur base.

5. Le fruit coupé horizontalement.

PI. CLXIII. Giroflier aromatique; Caryophylla aromatica.

a. Une fleur non épanouie, telle qu'on la livre au commerce, sous le nom de clou de girofle.

Pl. CLXIV. 1. La fleur avant son épanouissement.

2. La fleur complétement ouverte.

5. La même dépouillée de ses étamines.

4. Une étamine.

5. Le pistil coupé verticalement.

6. Ovaire coupé horizontalement.

7. Fruits avant leur maturité.

8. Fruil mur.

9. Fruit mur, vu par le sommet.

10. Fruit coupé transversalement, montrant en $a$ la loge contenant des ovules avortés.

11. Fruit coupé verticalement. $a$. Loge et ovules avortés.

12. Une portion du péricarpe très-grossi.

15. Fruit coupé horizontalement. $a$. Loge avorlée

14. Ovules avortés.

15 et 16. Embryons grossis.

17. Embryon dont on a écarté les feuilles cotylées.

18. Radicule. $a$. Coléorhize.

19. La même coupée.

Pl. CLXV. Loase à fleurs rouges; Loasa lateritia.

PI. CLXVI. Pourpier de Thelusson; Portuluca Thelussonii.

PI. CLXVII. 1. Cierge à cinq ailes; Cereus Pentalophus, var.

2. Échinocacte d'Otton; Echinocactus Ottonis.

Pl, CLXVIII. Café d'Arabie; Coffeca Arabica.

1. Calice et pistil.

2. Corolle étalée pour démontrer l'insertion des cinq étamines.

5. Le fruit de grosseur naturelle.

4. Le même dont on a enlevé une portion de l'épiearpe et du mésocarpe.

5. Le même coupé horizontalement.

6. Une graine.

7. La même coupée verticalement.

8. Embryon isolé.

9. Germination. $a$. Feuilles cotylées, épigées, accrescentes.

Pl. CLXIX. Cladanthe d'Arabie; Cladanthus Arabicus.

1. Calathide coupée longitudinalement.

2. Fleur accompagnée d'une squamelle et de quelques fimbrilles.

5. Périclinanthe et clinanthe.

4. Fragment de feuille grossie.

PI. CLXX. Tupa à fleurs roses; Tupa blanda.

1. Le pistil.

2. Le stigmate.

5. L'ovaire coupé transversalement pour montrer l'insertion des ovules.

PI. CLXXI. Rosage en arbre; Rhododendrum arboreum.

PI. CLXXIl. Épacride à grandes fleurs; Epacris grandiflora.

a. La corolle étalée et développée. b. Bractées.

c. Le pistil.

Pl. CLXXIII. Ahouai des Antilles; Cerbera Thevetia.

PI. CLXXIV. Martynie à trompe; Martynia proboscidea.

PI. CLXXV. Aschinanthe à grandes fleurs; Eschinanthus grandiflorus.

1. L'ovaire.

2. Le même coupé transversalement.

5. Le même coupé longitudinalement.

Pl. CLXXVI. Wigandie de Caraccas; Wigandia Caraccasana.

a. La corolle développée.

Pl. CLXXVII. Consonde du Caucase; Symphytum Caucasicum.

Pl. CLXXVIII. Brugmansie à deux couleurs; Brugmansia bicolor.

a. Une section de l'ovaire.

Pl. CLXXIX. Sauge à grandes fleurs bleues; Salvio patens.

Pl. CLXXX. Tunbergie de Hawtayne; Tunbergia Hawtayneana.

PI. CLXXXI. Cyclame à feuilles de Lierre; Cyclamen hedercefolium.

1. Le calice.

2. L'ovaire.

5. Le même coupé transversalement.

4. La fleur dépouillée de sa corolle et des sépales.

PI. CLXXXII. Nictage Faux-Jalap; Nictago Jalapa. On a réuni sur la même tigge diver'ses variétés de couleur.

PI. CLXXXIII. Begonie sanguine; Begonia sanguinea.

Pl. CLXXXIV. Aristoloche à fleurs labellées; Aristo. lochia labiata.

Pl. CLXXXV. Médicinier à feuilles entières; Jatropha integervima.

Pl. CLXXXVI. Garrye à feuilles elliptiques; Garrya elliptica.

P1. CLXXXVII. Jacquier à pain ; Artocarpus incisa.

1. Chaton mâle;

2. Chaton femelle.

3. 4. Fleurs mâles

5. Fleur femelle.

6. Une graine avec sa tunique développée.

7. Le fruit ou chaton coupé verticalement.

\section{Monocotylédones.}

PI. CLXXXVIII. 1. Cocotier ordinaire; Coco nucifera.

2. Bananier du Paradis; Musa Paradisiaca.

3. Canamelle ou Canne à sucre; Saccharum officinarum.

4. Iris germanique; Iris germanica.

5. Narcisse des poëtes; Narcissus poeticus.

6. Cymbidier à fruit hérissé; Cymbidium echinocarpon.

7. Épidendre de Chine; Epidendrum Sinense.

Pl. CLXXXIX. Gouet campanulé; Arum campanulatum.

1. Une feuille.

2. Le pistil.

ธ. L'ovaire.

4. Le même coupé transversalement. 
PI. CXC. Oncidier à feuilles triangulaires; Oncidium triguetrum.

1. La fleur vue en dessous.

2. Le labelle.

3. Le gynostème en colonne.

4. L'anthère.

5. Les deux masses polliniques.

Pl. CXCI. Amome gringembre; Amomum Zinziler.

1. La tleur grandie.

2. Le filet de l'étamine fortement grossi.

5. L'ovaire.

4. Le même coupé transversalement.

5. Une graine.

6. La même coupée verticalement, montrant en $a$ l'embryon.

Pl. CXCII. Glayeul (Antholize) d'Éthiopie; Gladiolus Ethiopicus.

P1. CXCIII. Habranthe robuste; Habranthus robustus. Les étamines, le style et le stiggmate dans leur position réciproque.

Pl. CXCIV. Amaryllis réticulée; Anaryllis reticulata.

1. La corolle développée, pour montrer la position du pistil et des étamines.

2. Une anthère grossie.

P1. CXCV. Cyrtandre à longutes étamines; Cyrtandra staminea.

a. Le calice, avec une partie de la corolle, grossis. $b$. Le pistil fortement girndi. c. La corolle développée et grandie.

d. Une étamine fort grossie.

e. Anthère très-grossie, vue par le dos.

$f$. Le fruit.

g. Le même coupé transversalement, arec trois graines isolées.

i. Un poil cloisonné, fortement grossi.

Pl. CXCVI. Alstrœmérie Perroquet; Alstræmeria Psiltacina.

Les six premières figures d'analyse montrent chacum des pétales, auquel adhère à sa base l'étamine.

La septième figure est le pistil.

La huitième présente un fleuron stérile.

Pl. CXCVII. Pitcairnic à feuilles étroites; Pitcaimia angustifolia.

PI. CXCVIII. Lis élégant; Lilium speciosum.

P1. CXCIX. Saģouier farinifère; Sagus farinifera.

1. Portion d'un régime de fleurs.Grandeur naturelle.

2. Fleur fertile.

5. Fleur stérile.

4. Le fruit.

PI. CC. Calectasie à fleurs bleues; Calectasia cyanea.

La première figure d'analyse représente le pistil grossi; la suivante et celle qui lui correspond dc l'autre côté de la plante, sont des étamines grossies et dessinées sous divers aspects.

La troisième figure est celle d'une feuille grossie, dont une section transversale montre la coupe. 


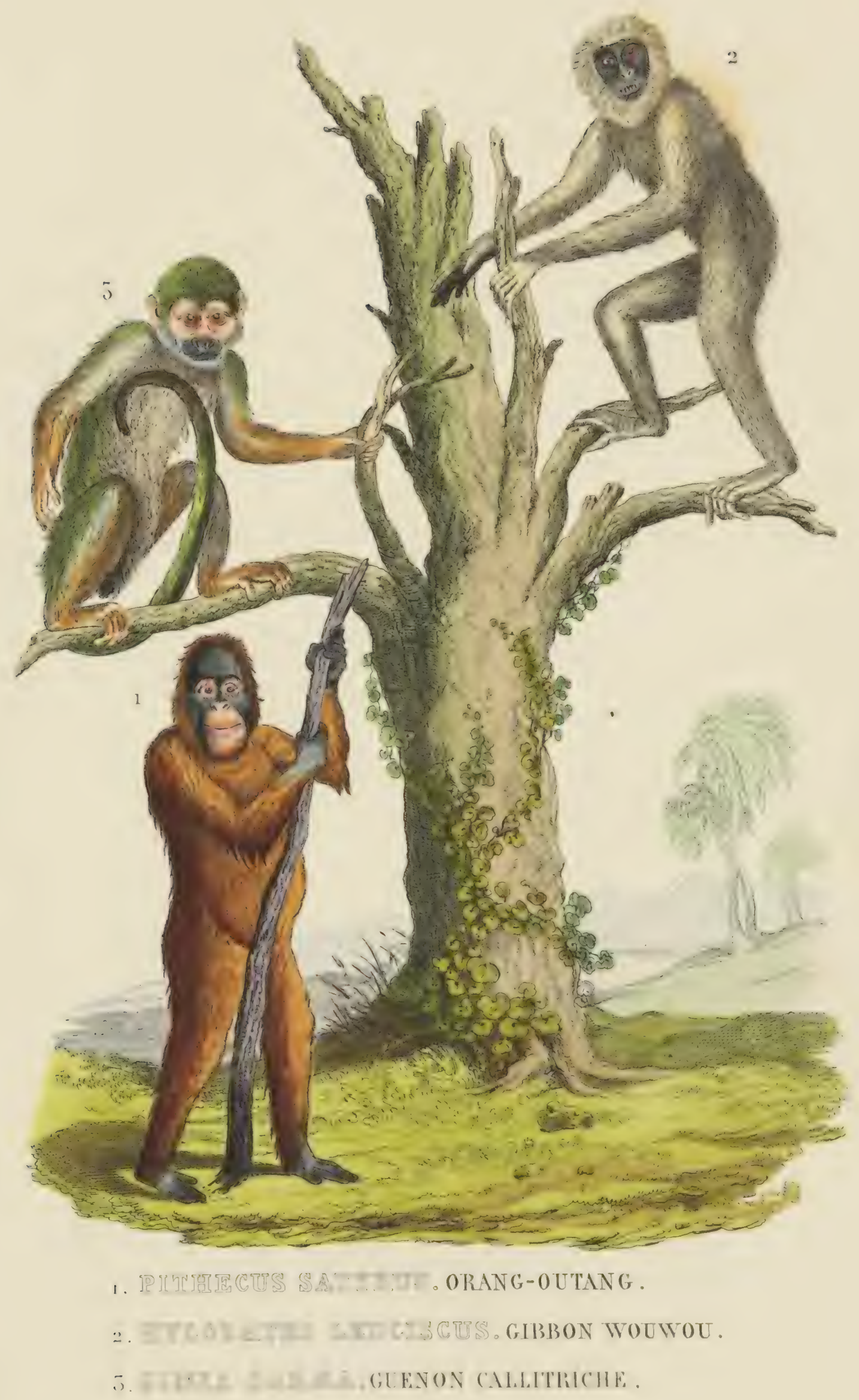




$$
\text { 鯂 }
$$



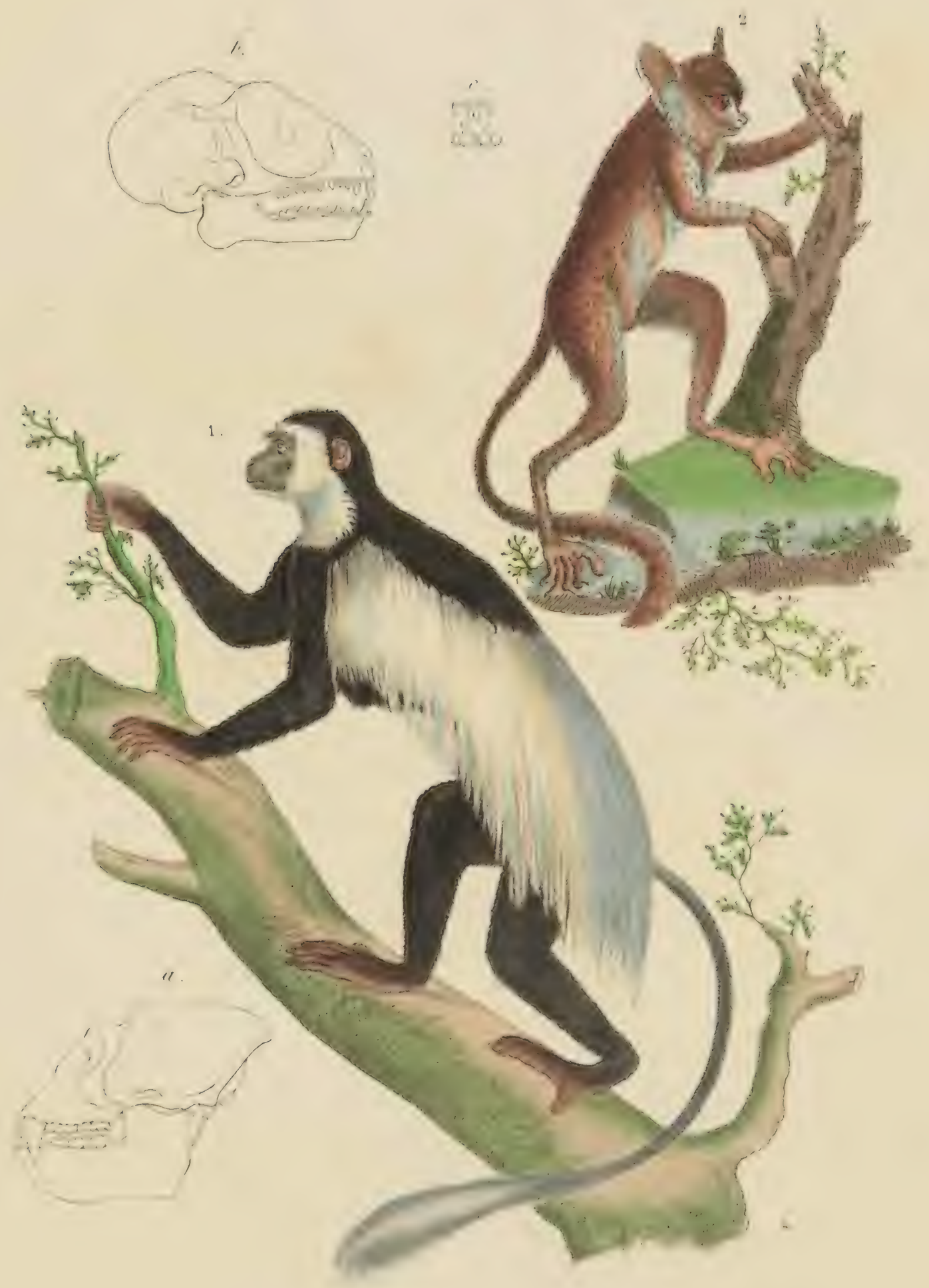

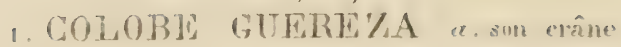

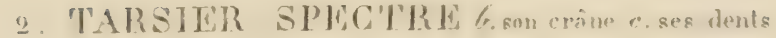




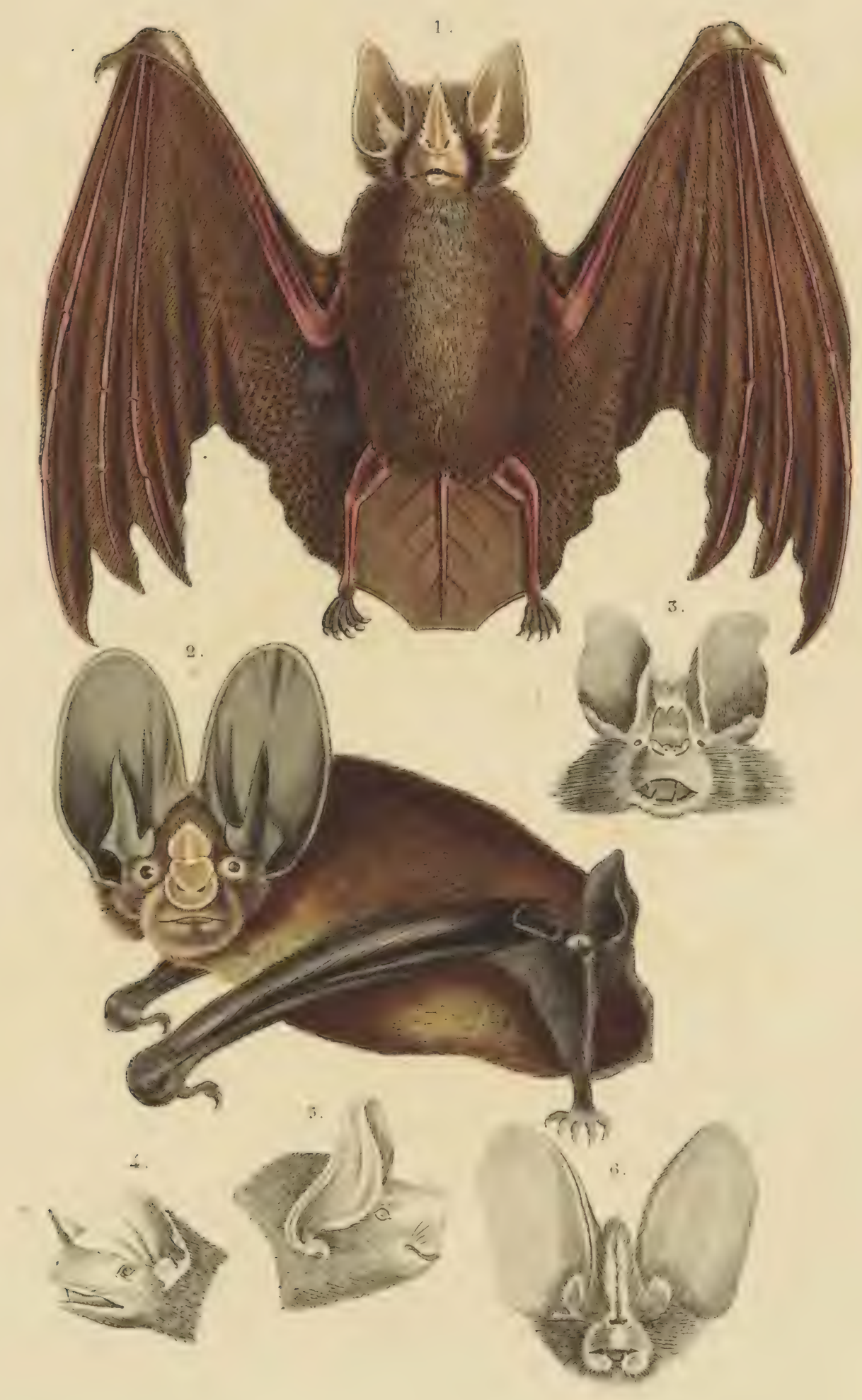

1. PIIYli,OSTOALE CRÉTELÉ. ́. MOLOSSE OBSCUR.

\&. MÉGADERVE TRETLE. 5. NOCTLLION LIETRE.

3. RIINOHOPIE T'RTDENT. G. NYC'LLRE DF GEOFHROY. 


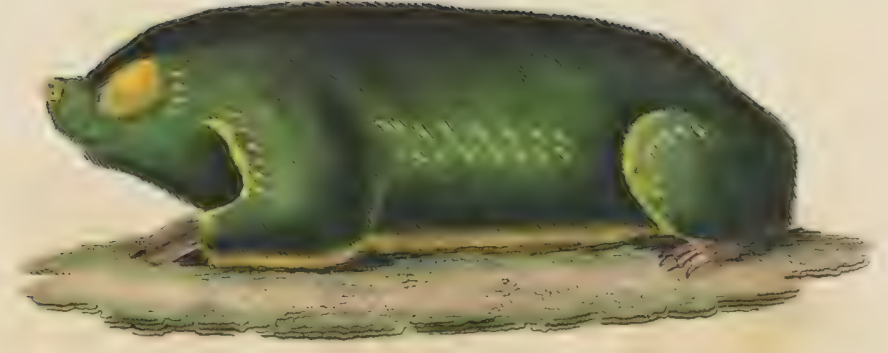

$\therefore$

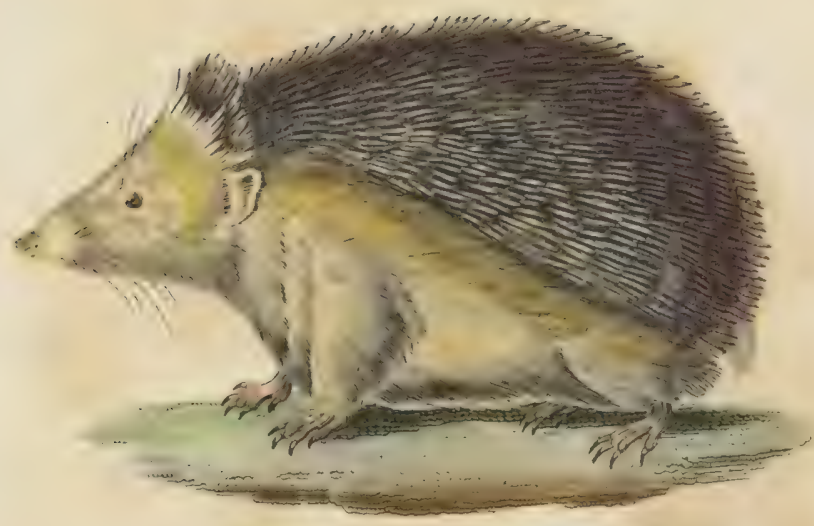

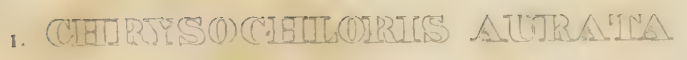

CHRYSOCHLORE DU CAP.

2. IV TE Th- TIM

IIÉRISSON COMMUN. 


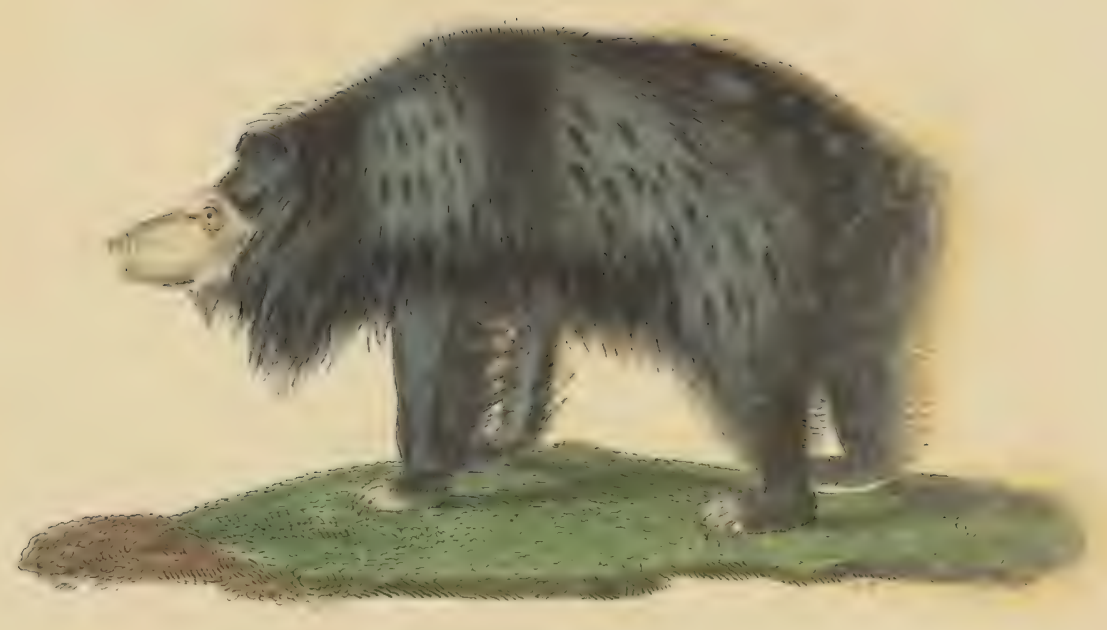

2

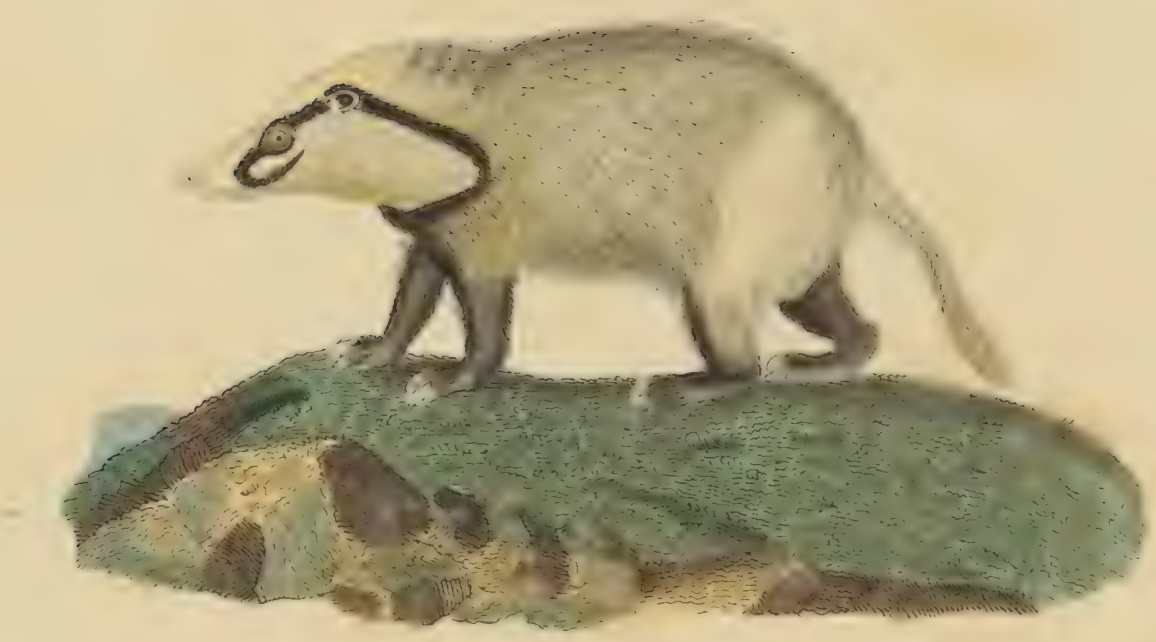

1. TTLSSTS TUANBICATUES

OERS AUX GRANDES LEVTRES

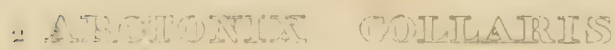

BNILT-SAUTR A COILIER 

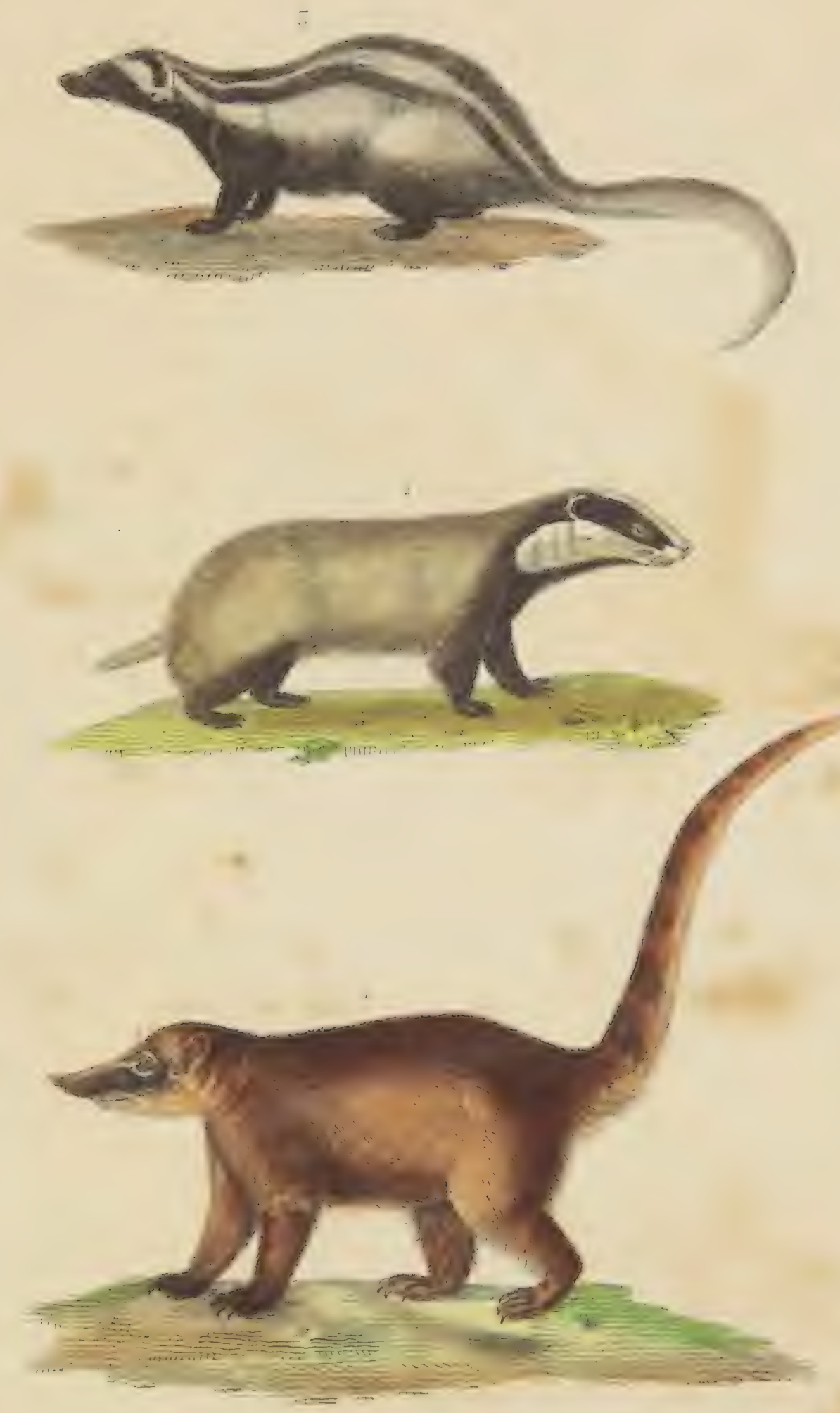

.

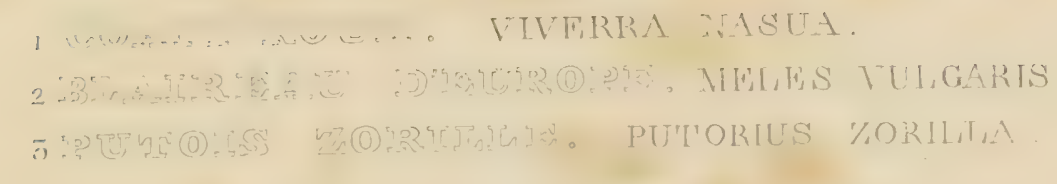



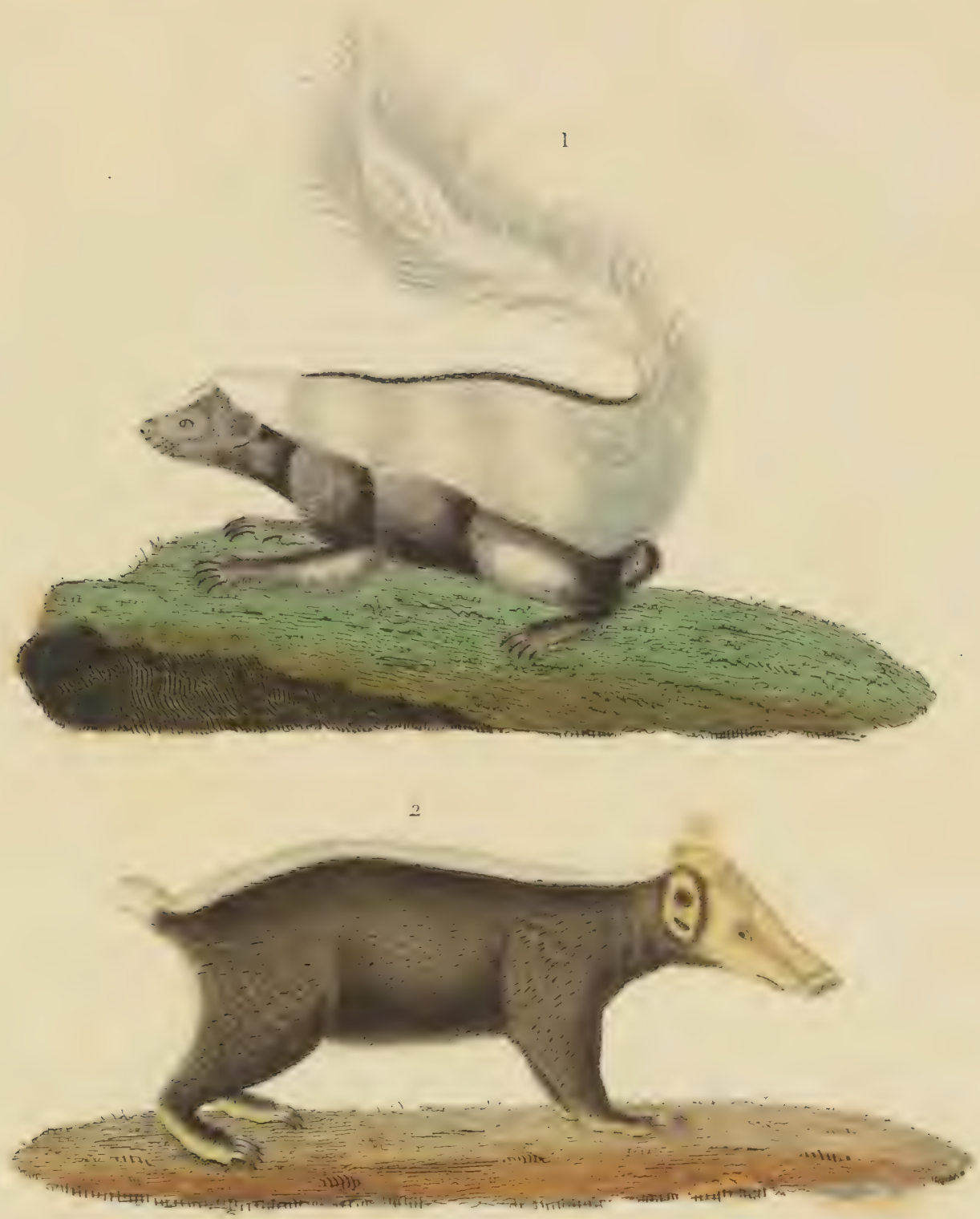

(I)

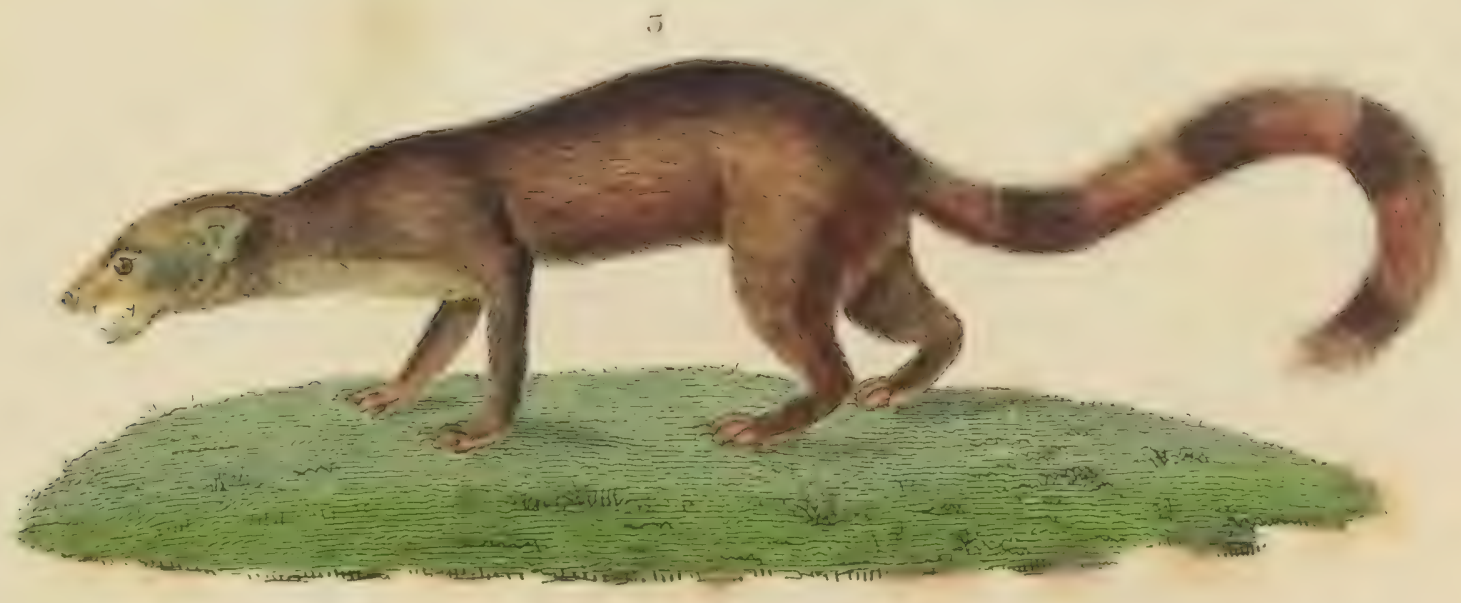

1. MOUHEETTE CHINCHE. Mephitis.

2. MYDAS TÉLAGON. Mvadaus meliceps.

-. GATIDIE ÉLEGANTE. Galidia elegans. 

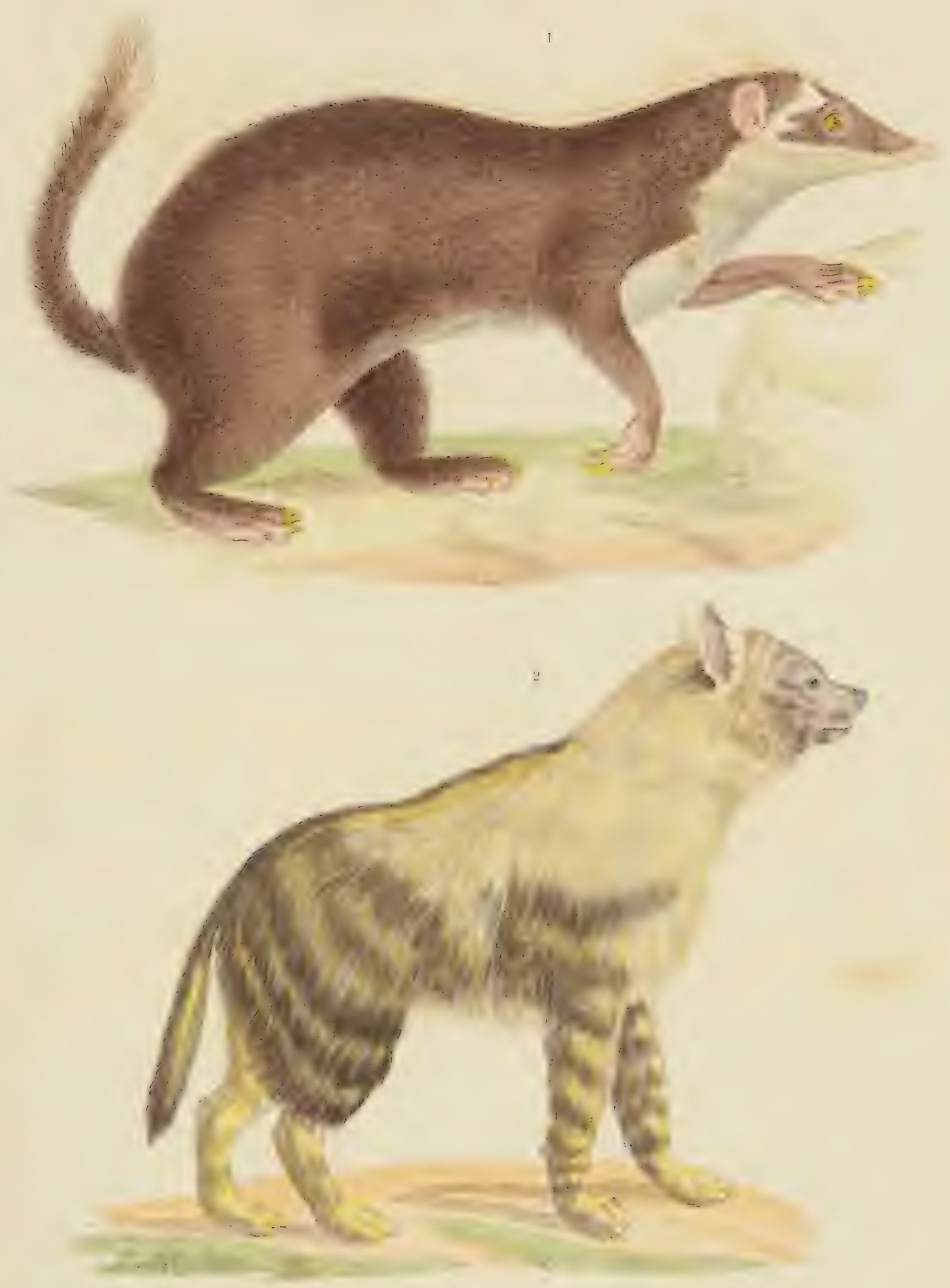

1. MELOGALE JRUNE.

2. HYYXW BRUNE. 

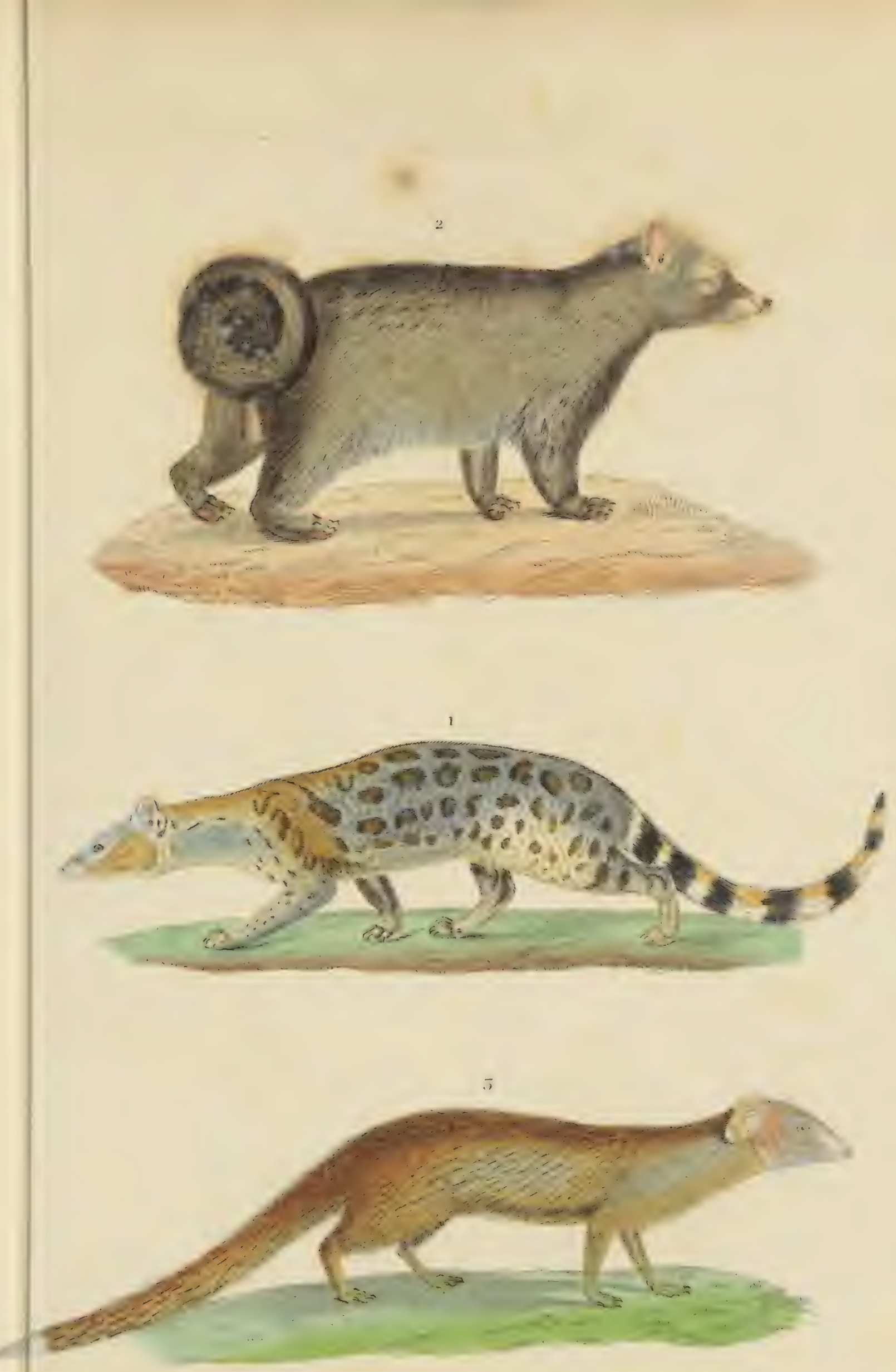

1. CTVETTE A BANDEAL. Viverra fasciata.

2. PARADOXURE TYPE. Paradoxurus typus

5. ICHNEUME ALBESCENTE. Ichneumia albescens. 


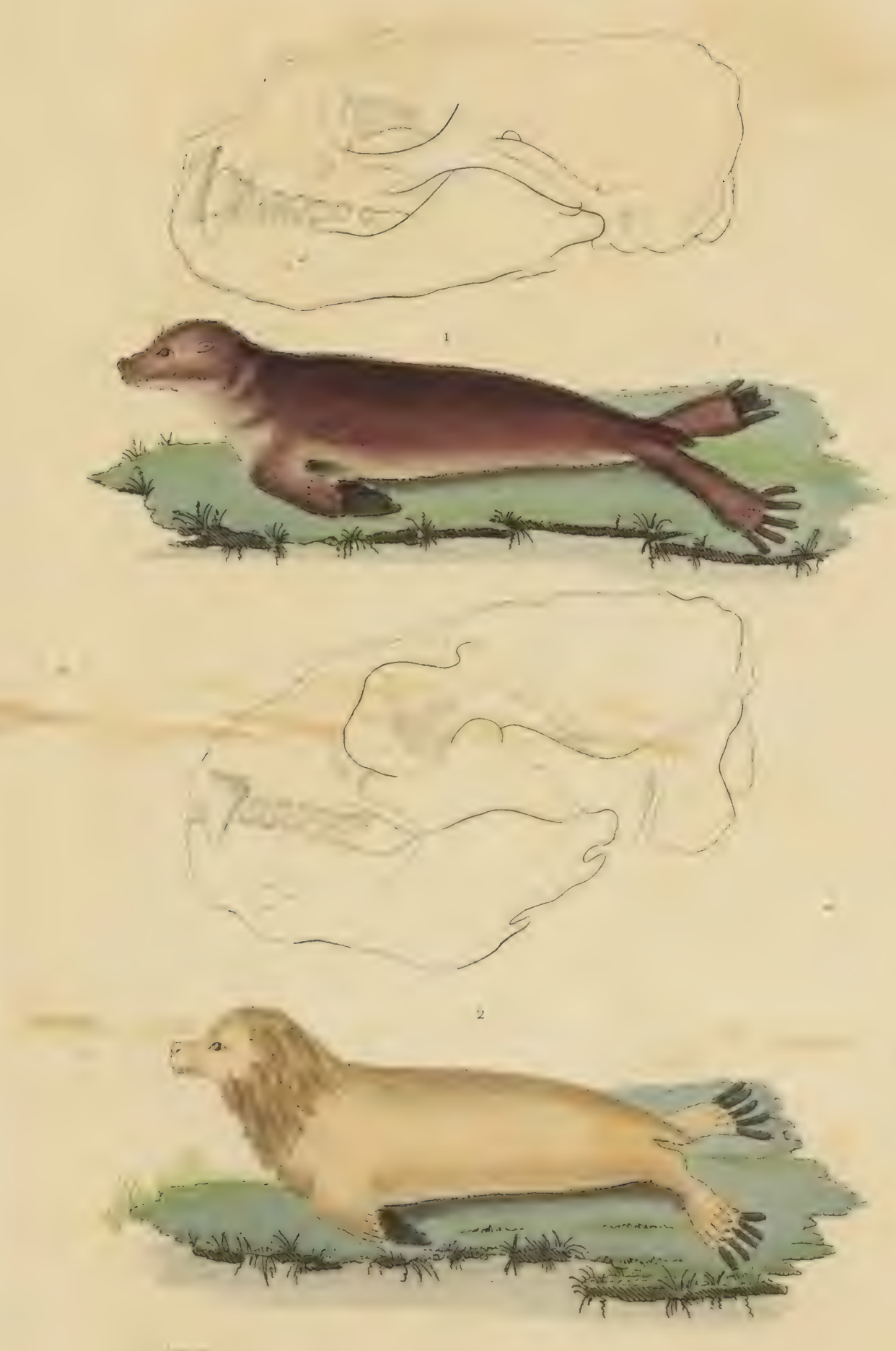

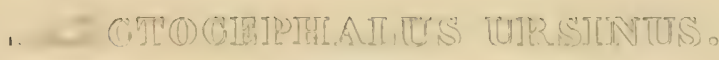

PHOQUE OURS MARLN.

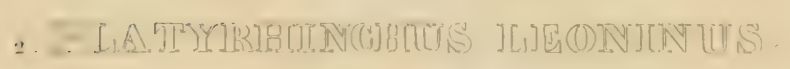

PHOQUE LION MARIN. 


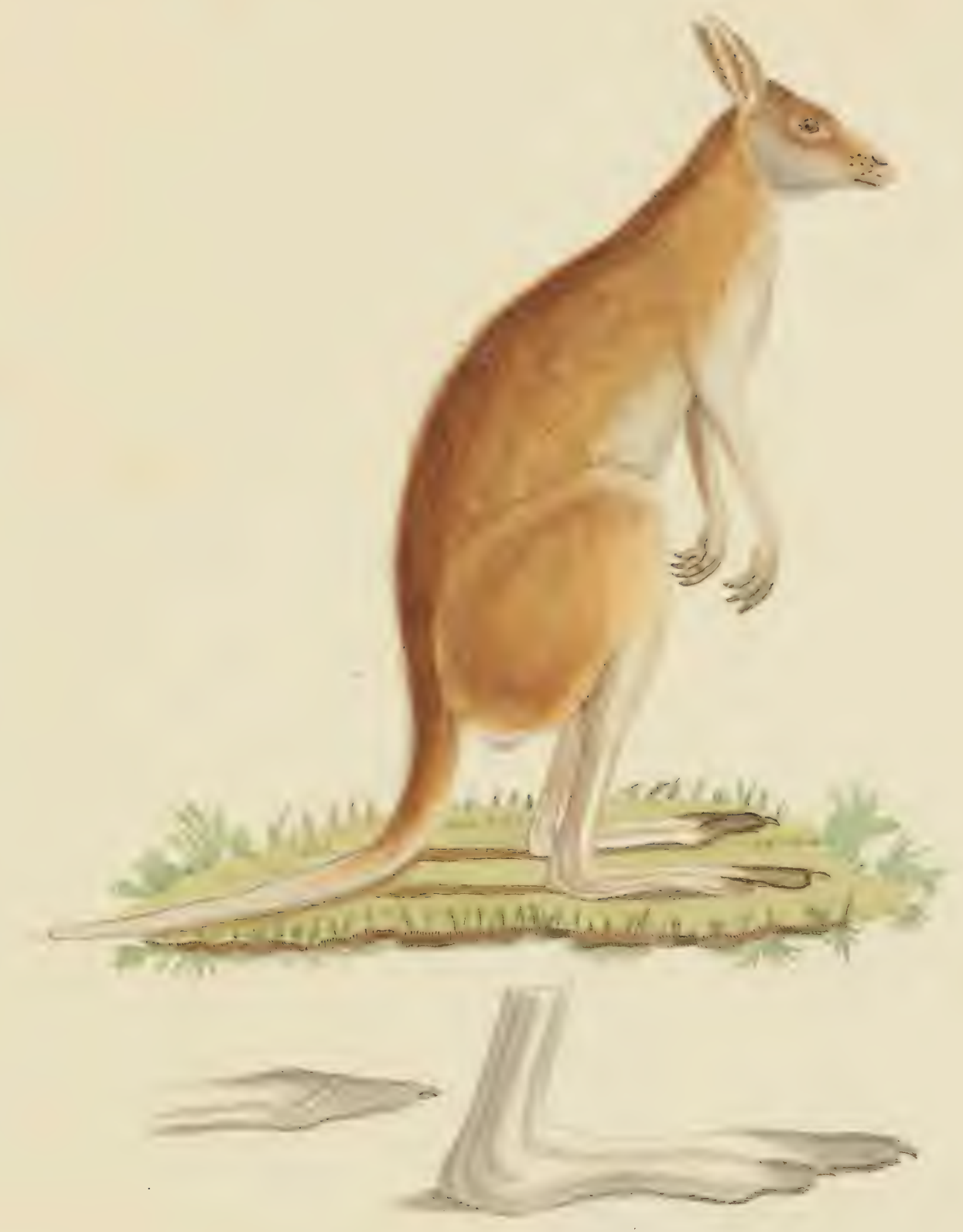

IKANGUTRUS IANIGLP

KATGUTOO TAINEUA. 


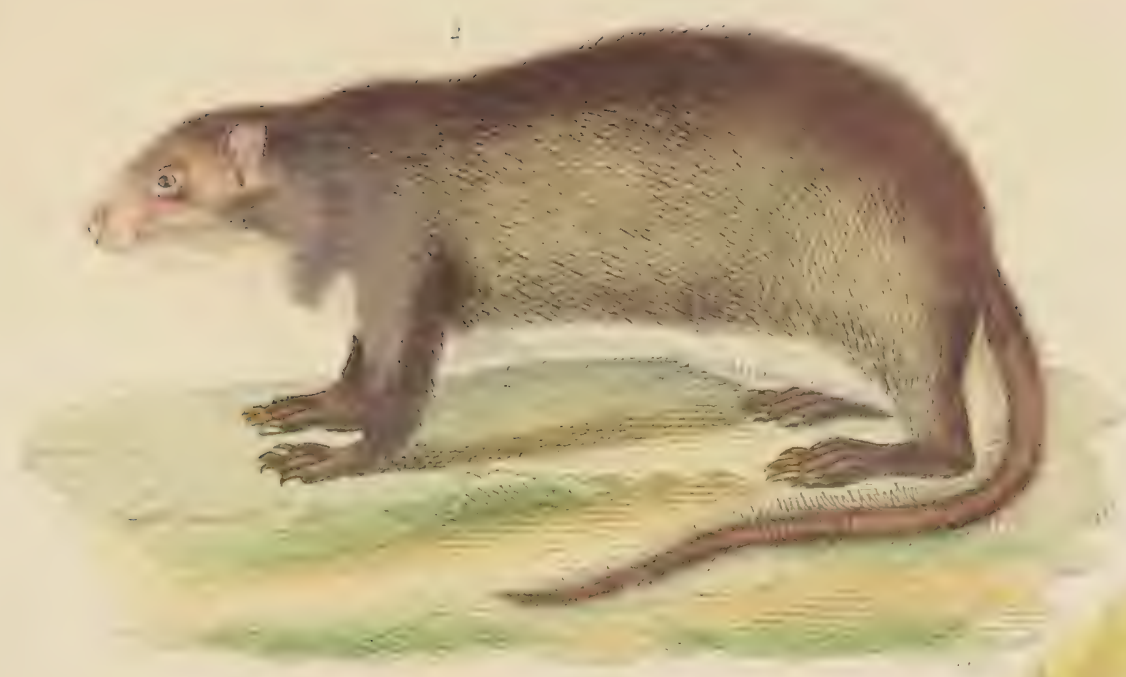

$\therefore$
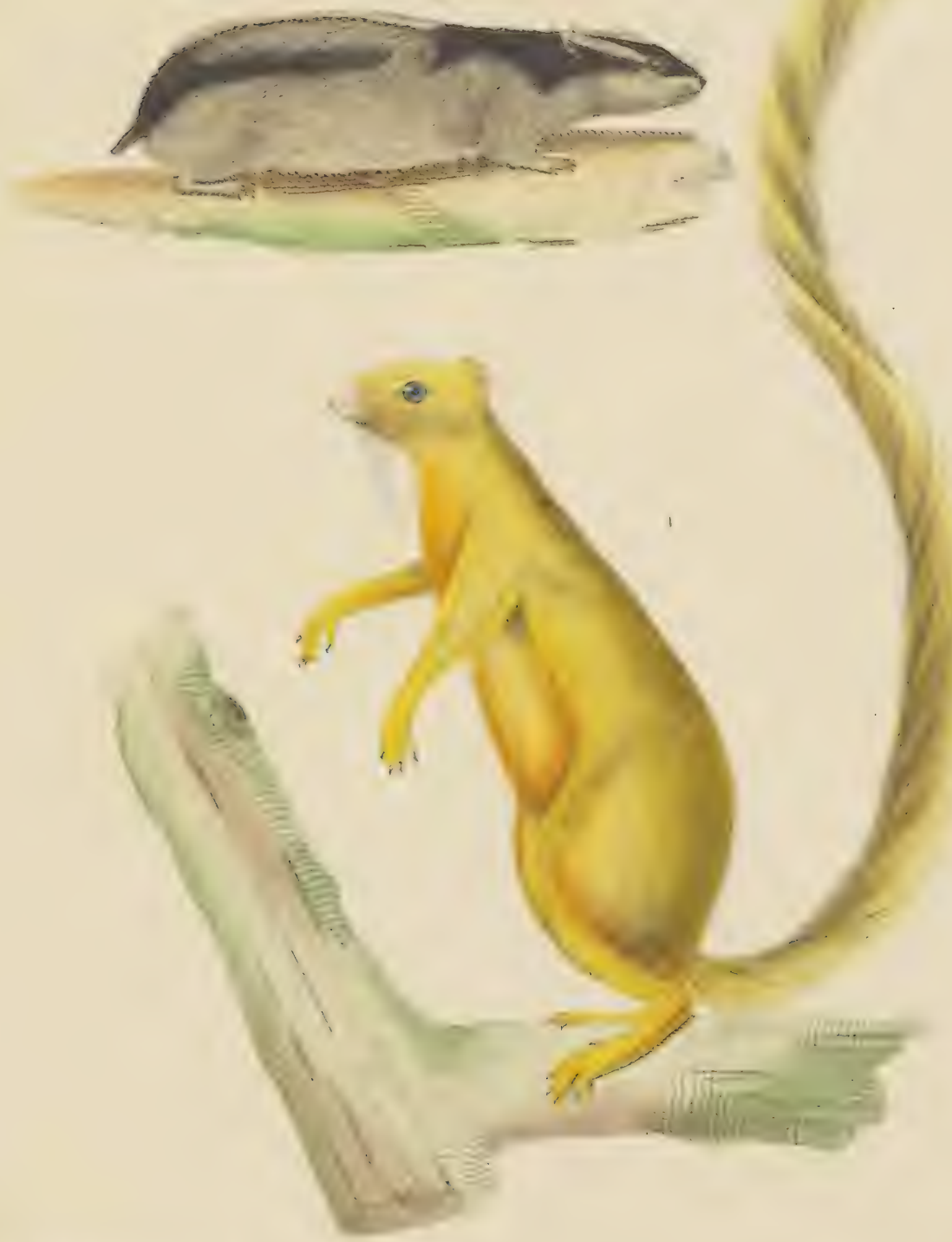

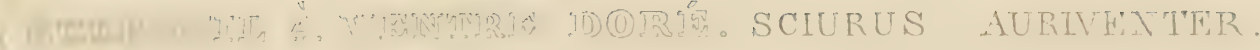

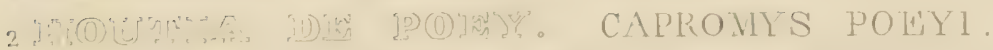

5y, 

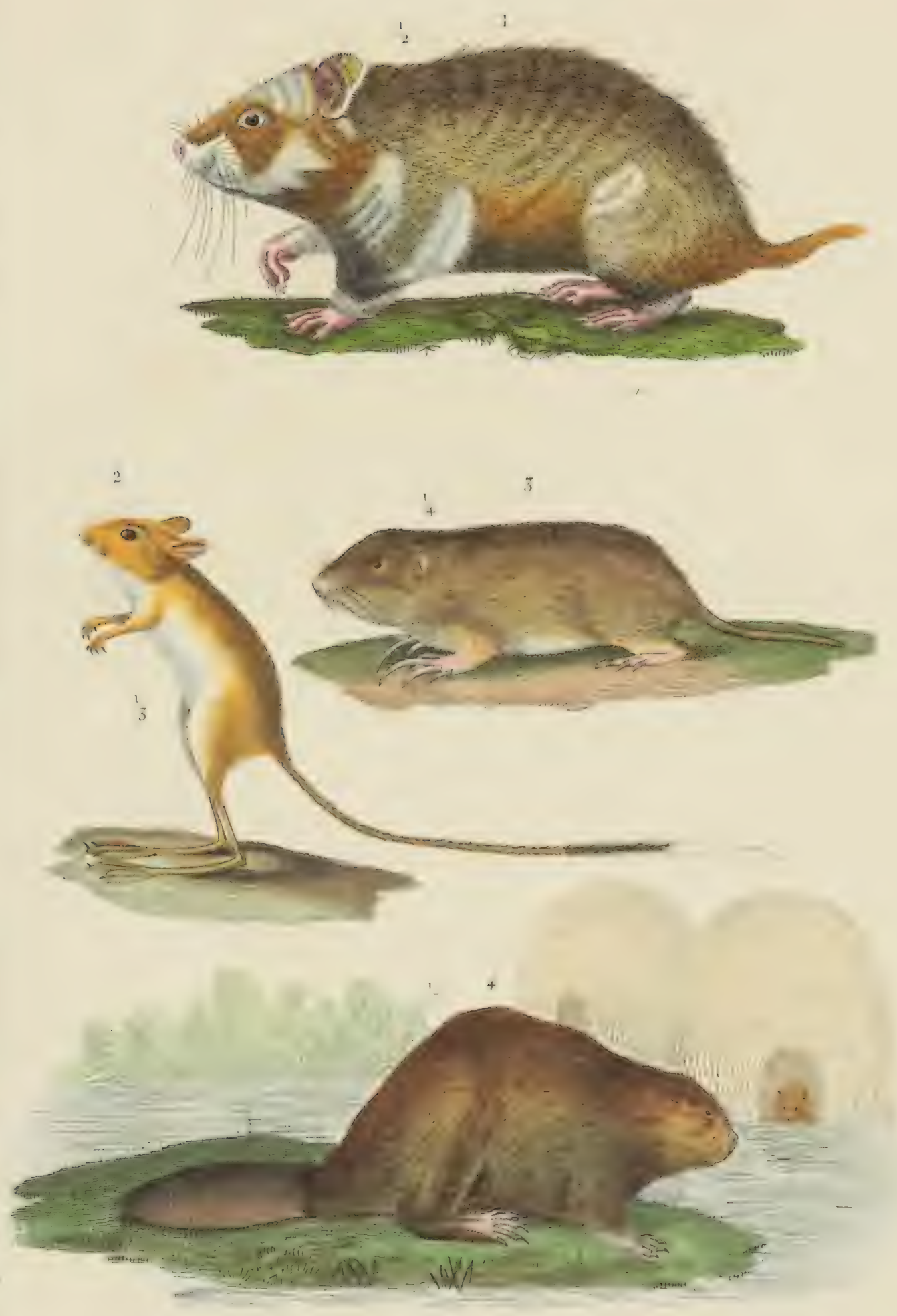

1. HAVS TTER commun

2 GERBOISE alactaga

5 SACCOMYDE anthophite

+. CASTOR du canada. 

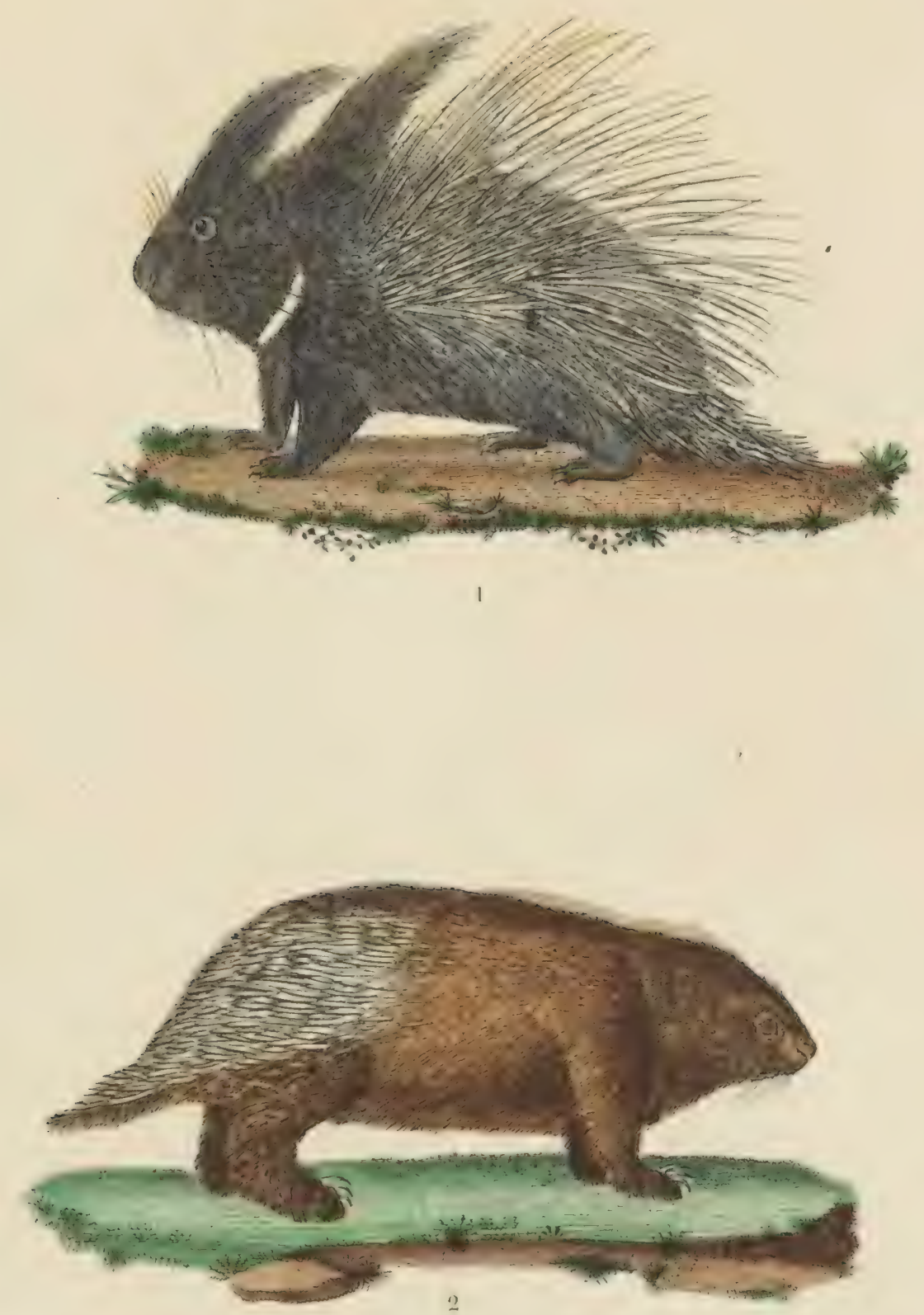

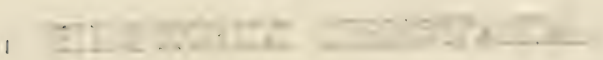

PORC-FirC D'TRALIE

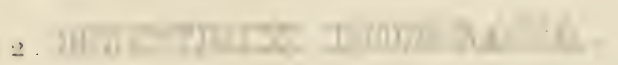

L:TTRSON 

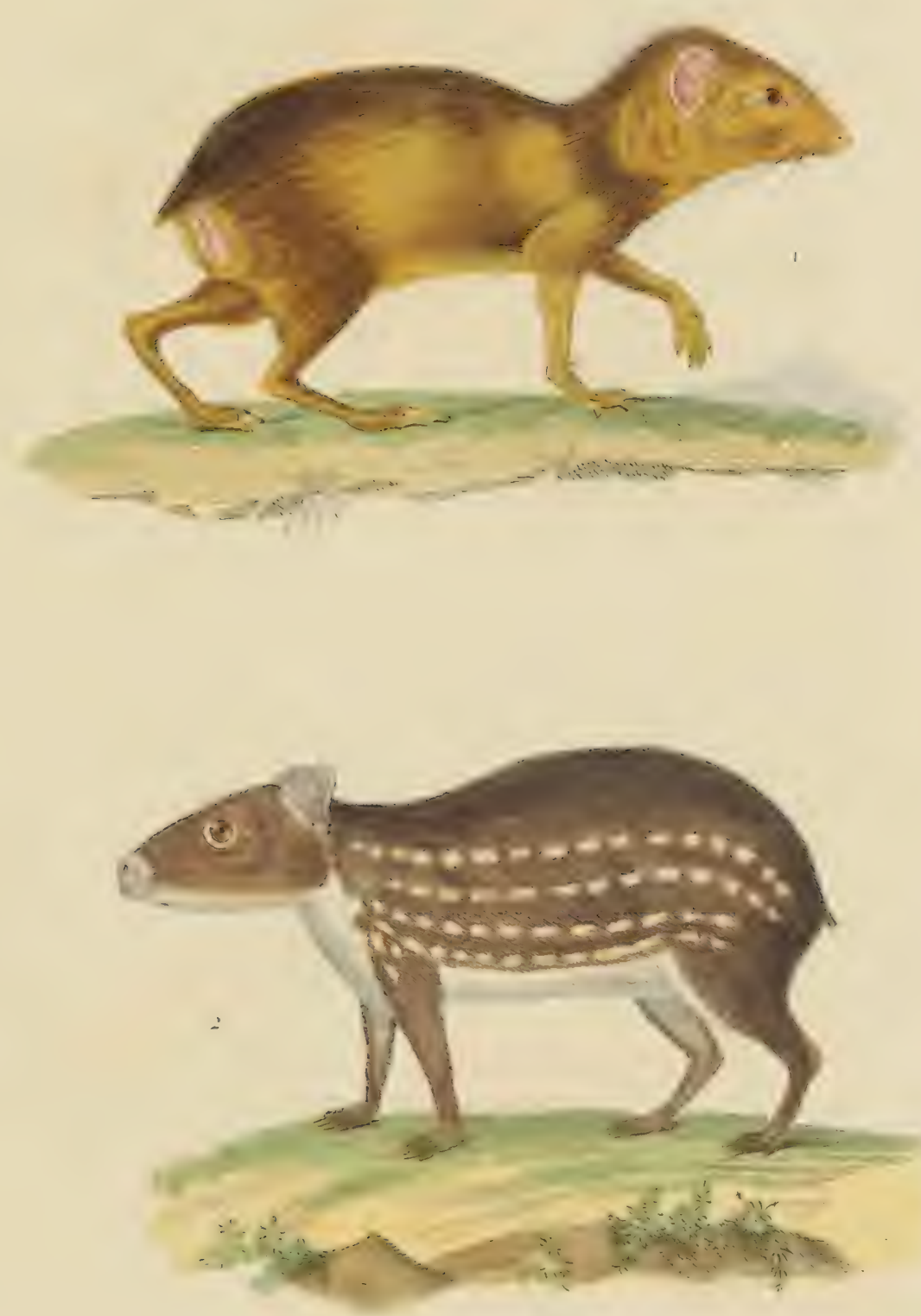

1. -74

I(i) (1)

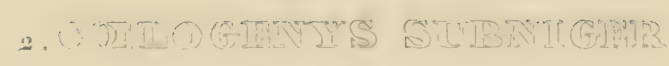

PACA. 


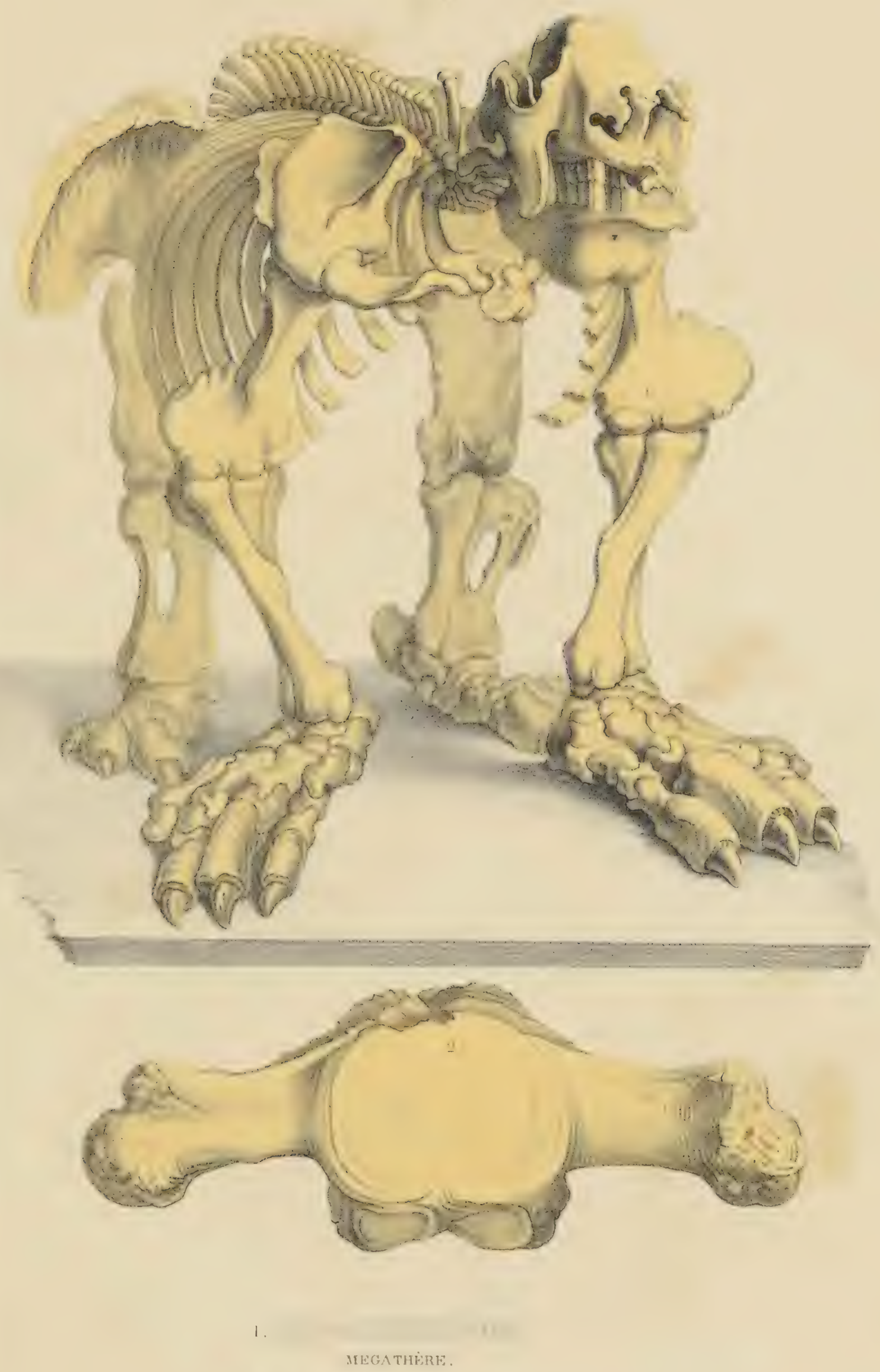

2. Ine de ses mertobres condules. 


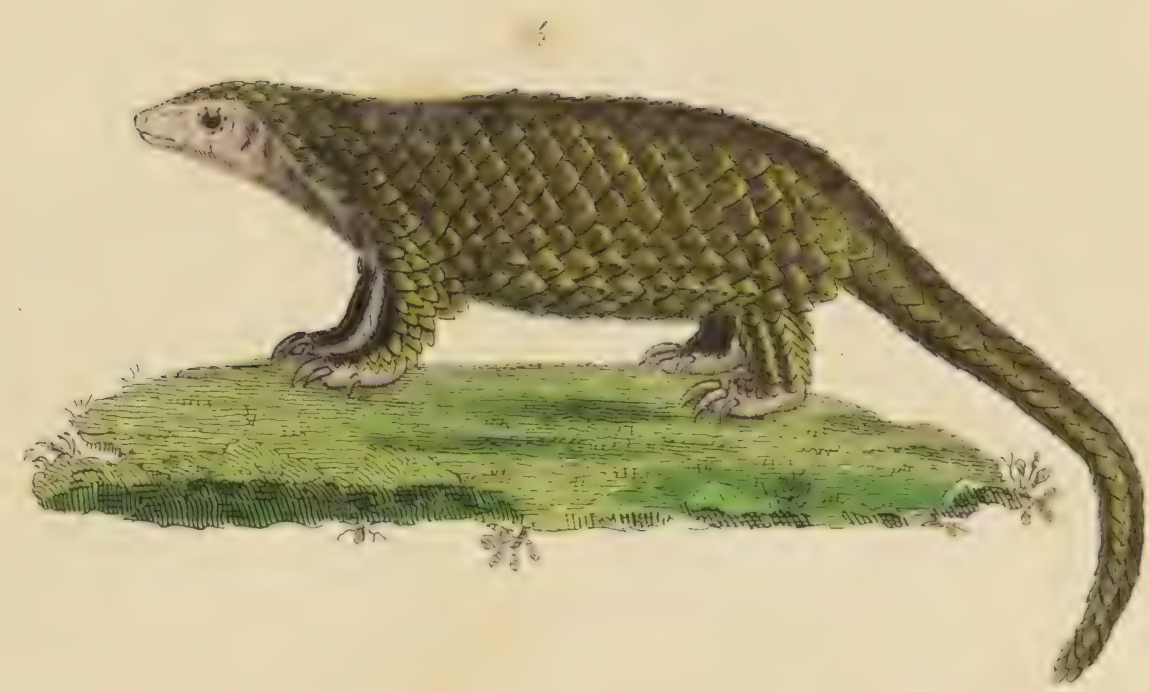

2
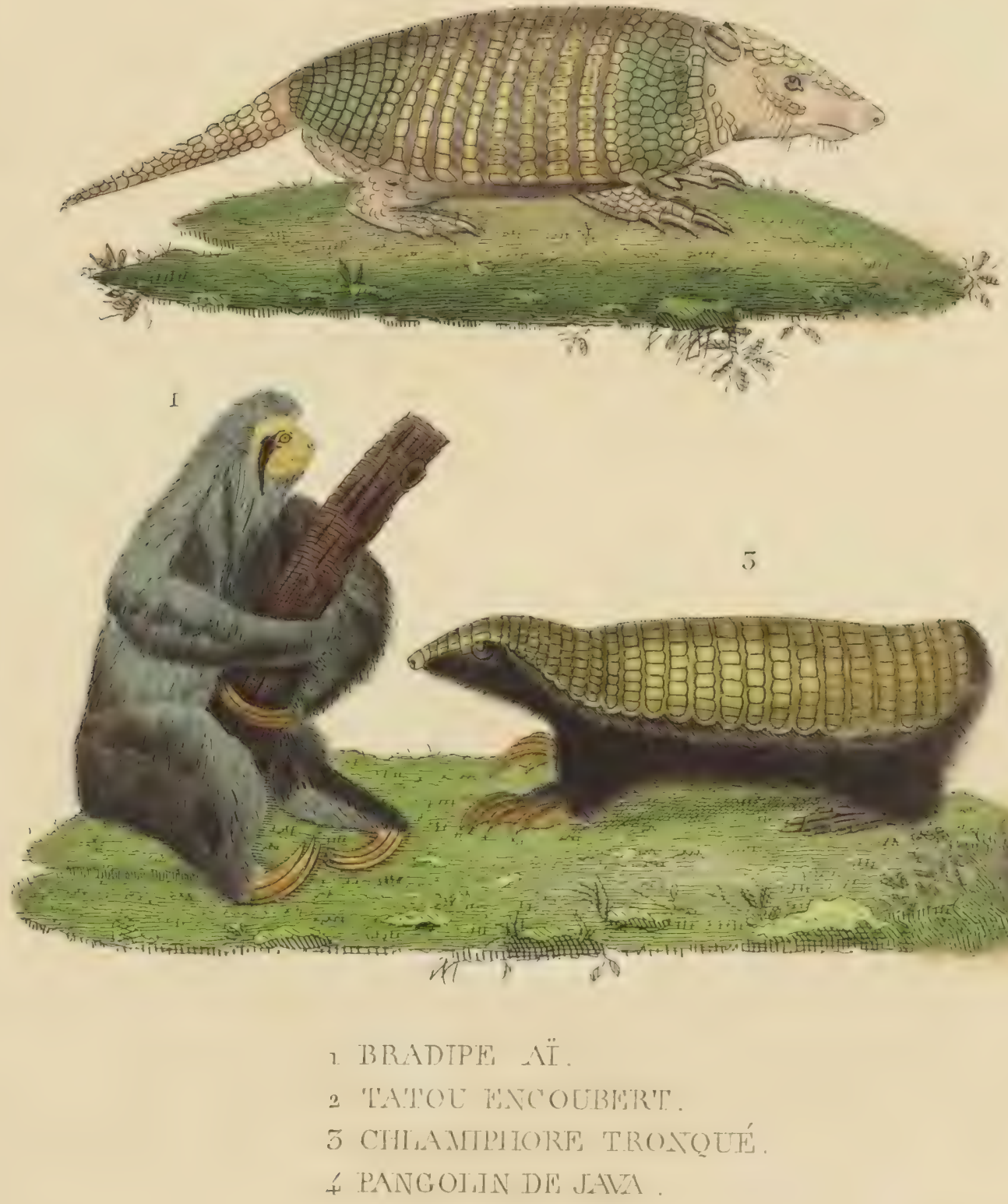

5

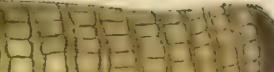




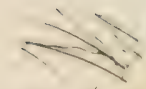

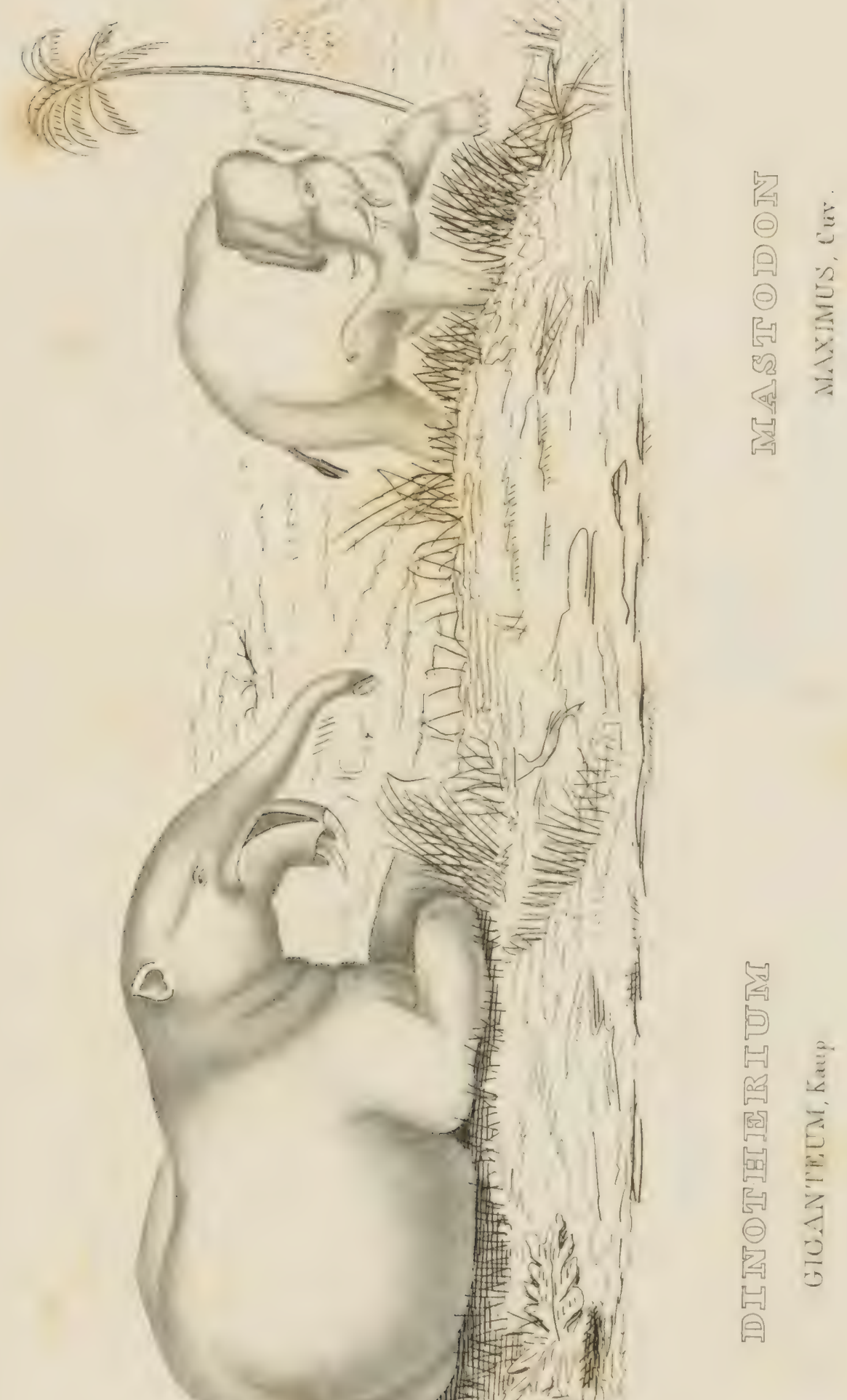



5
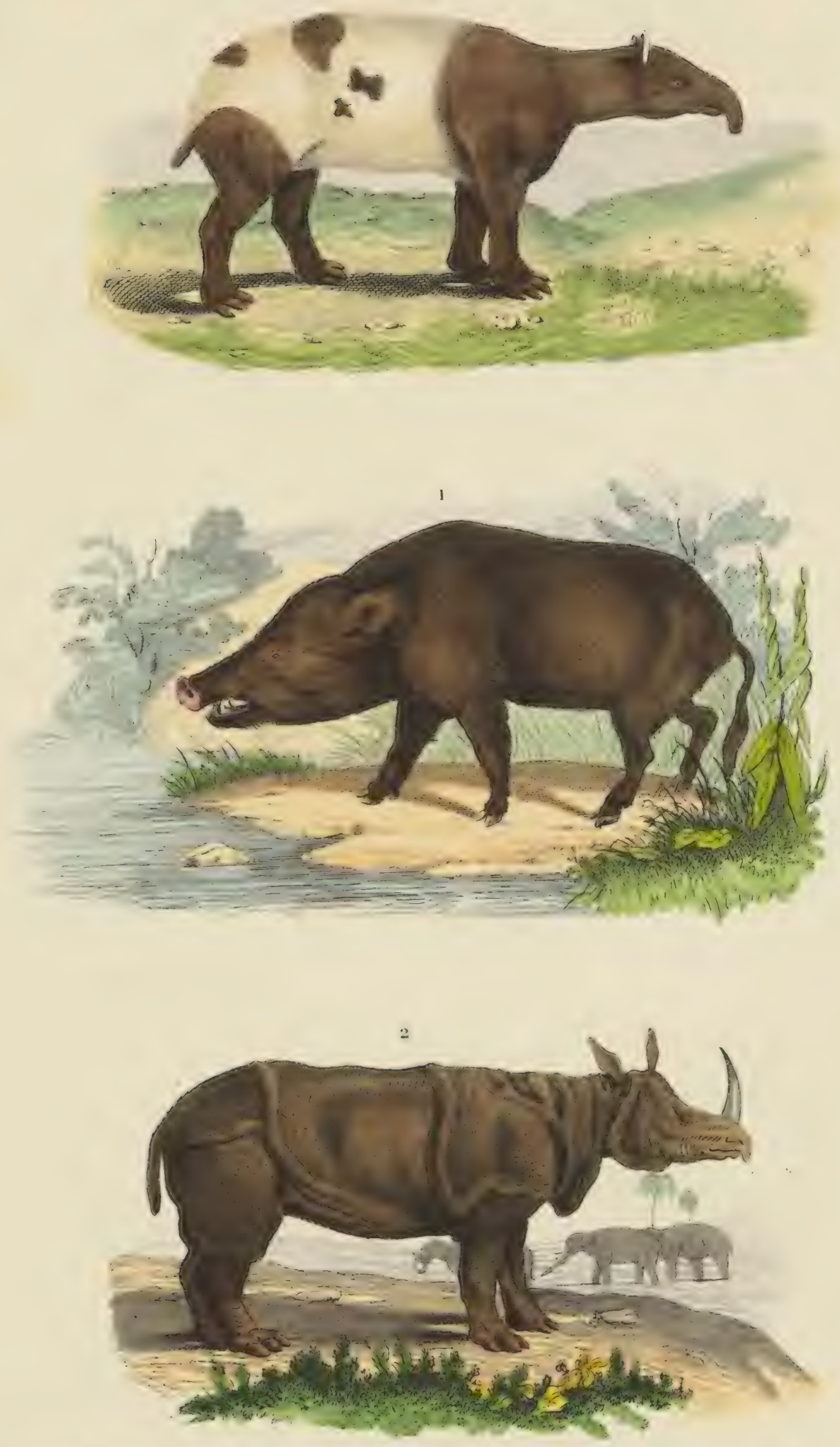

COCHON SANGLARR.

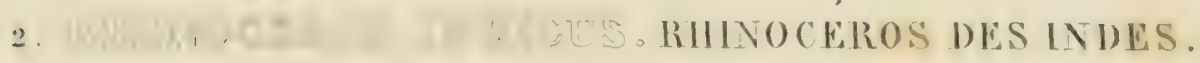

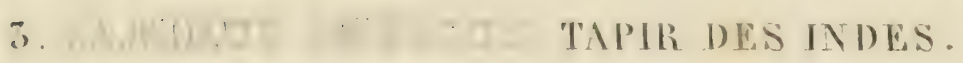




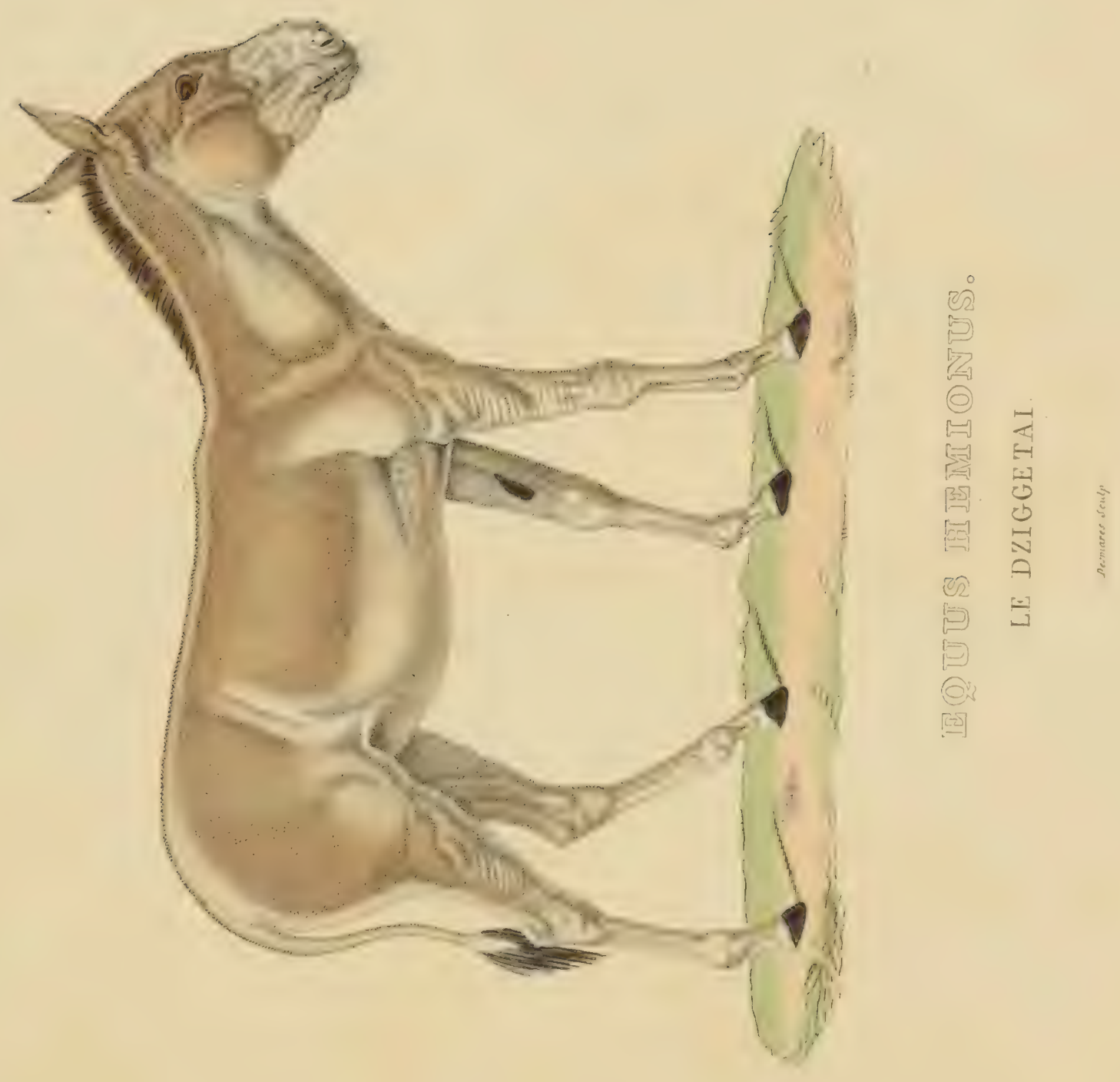




$$
\frac{\pi}{\pi}
$$




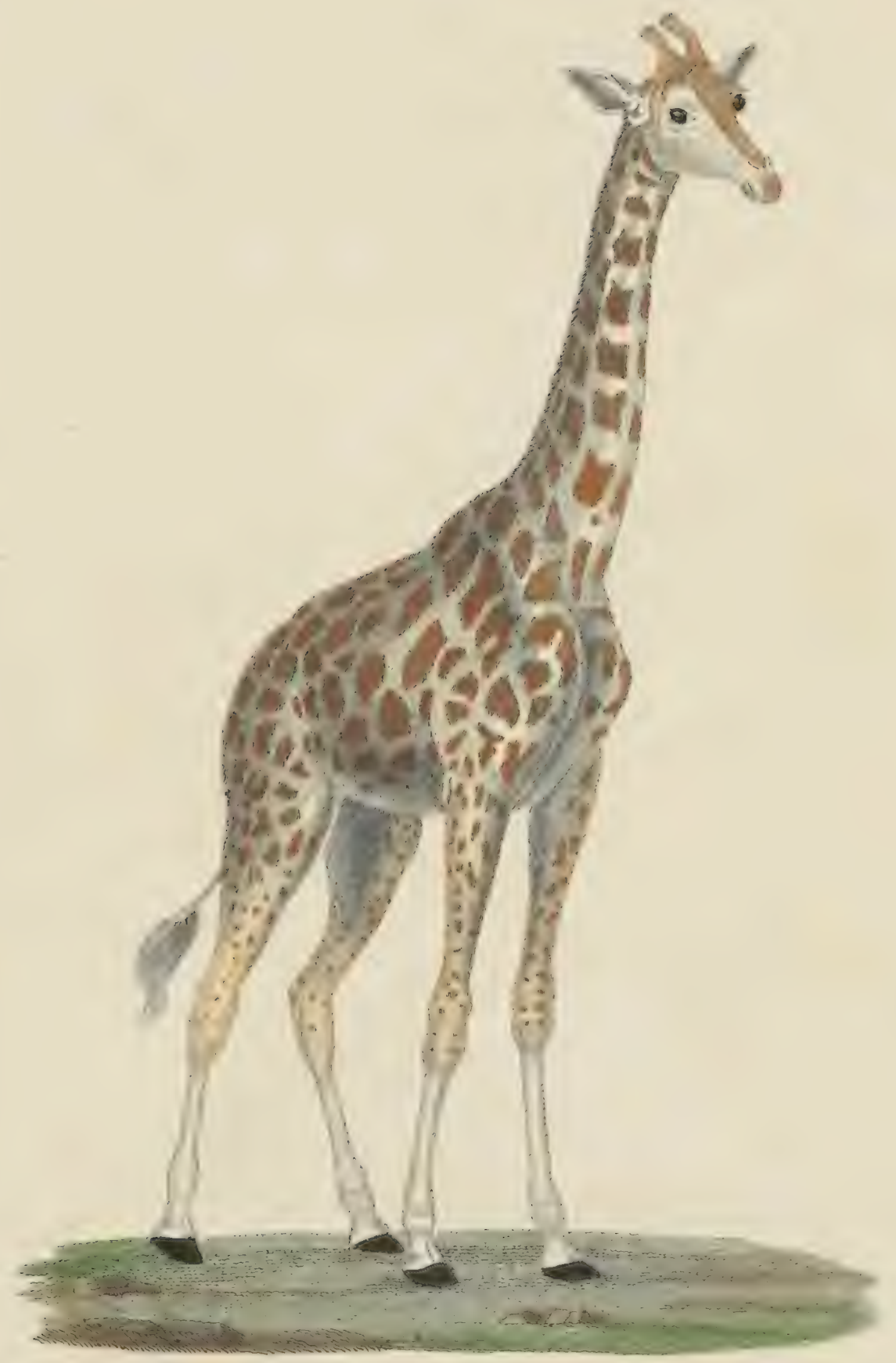

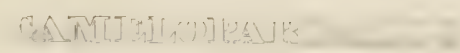

(IIRAFE. 

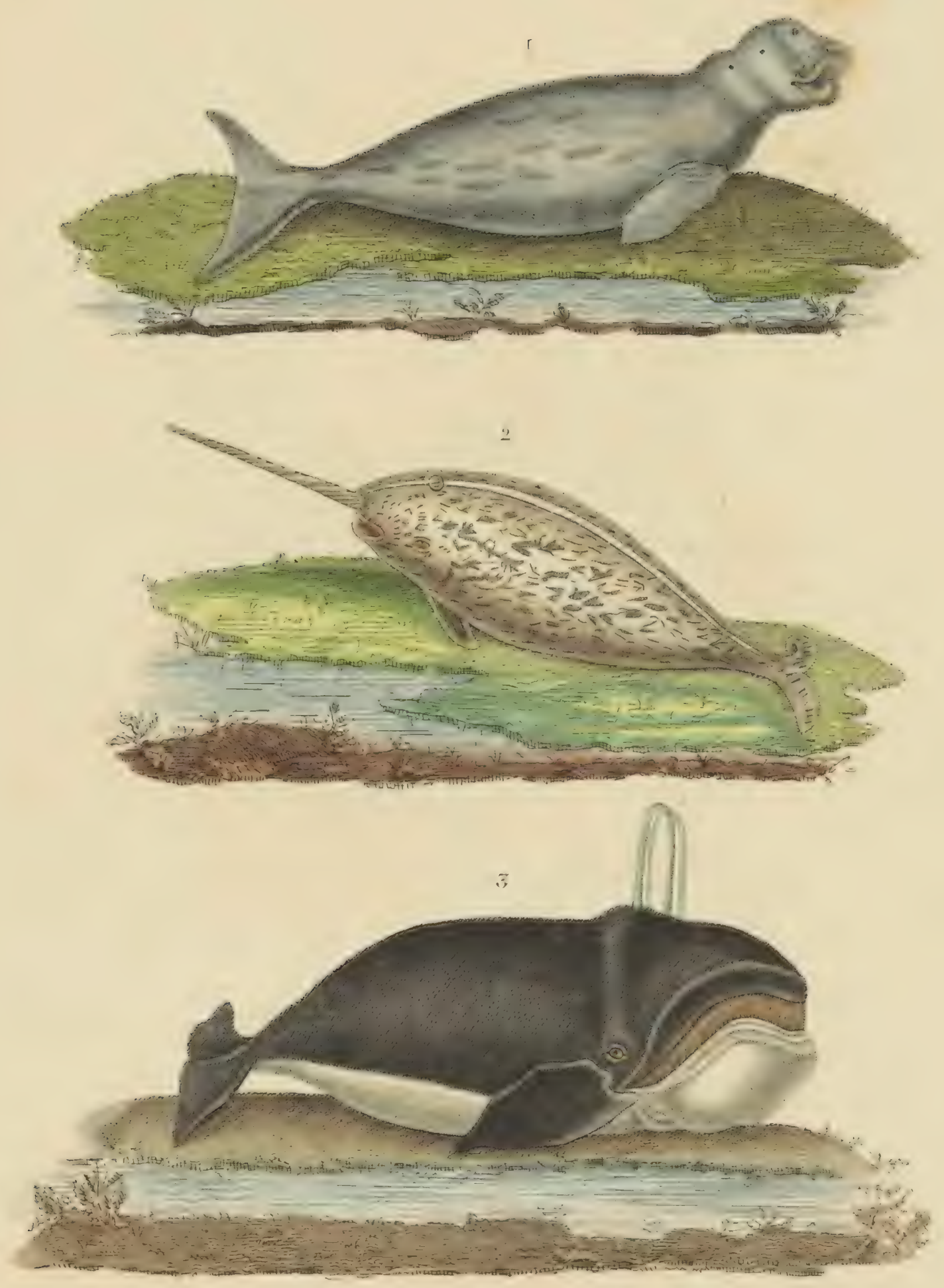

[ DUCGNG. 'Trichecus Durong.

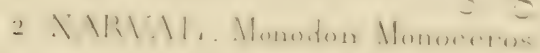

3 BALEINE ERATCHH, Baloena Mrsticetus. 


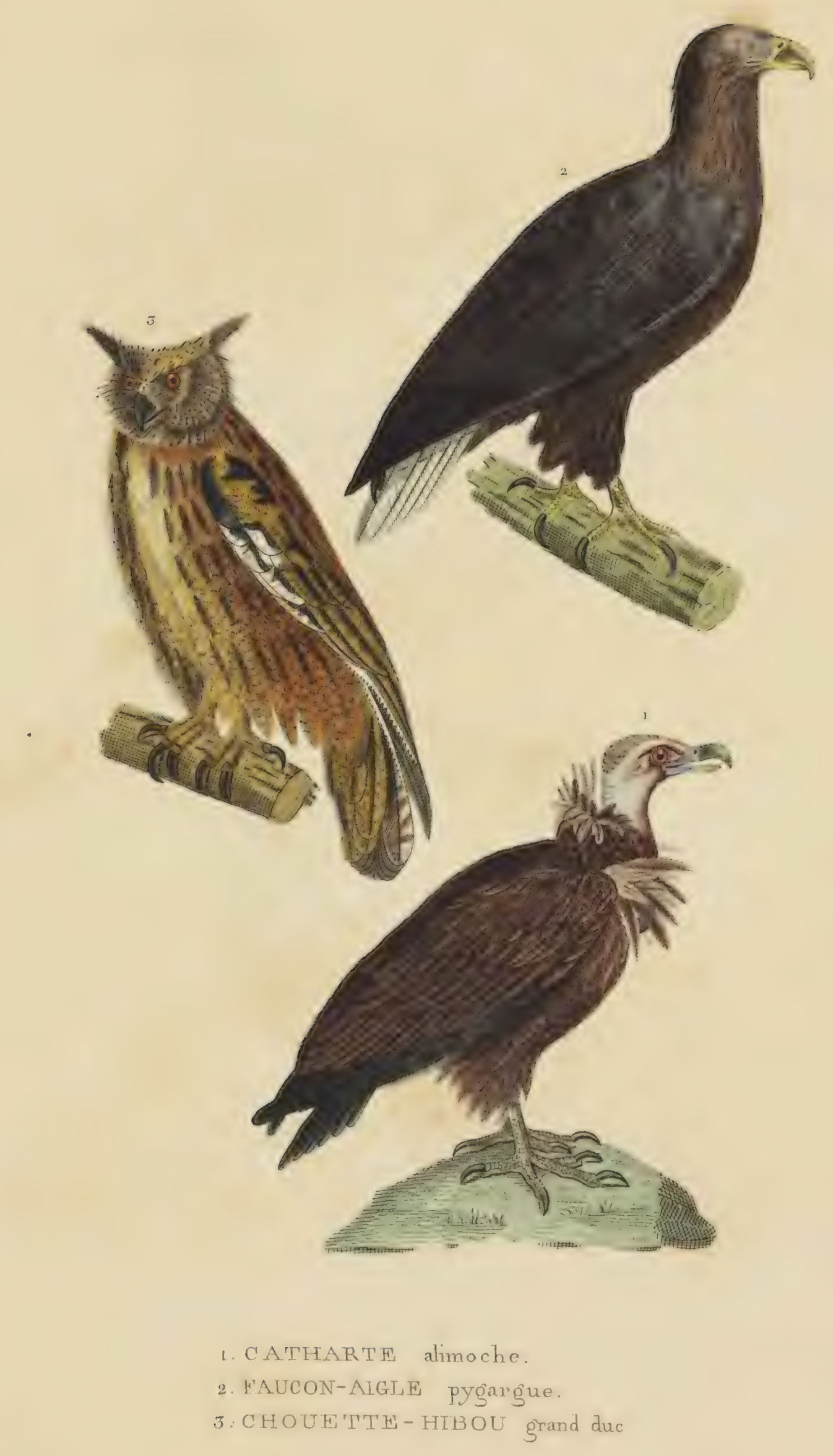




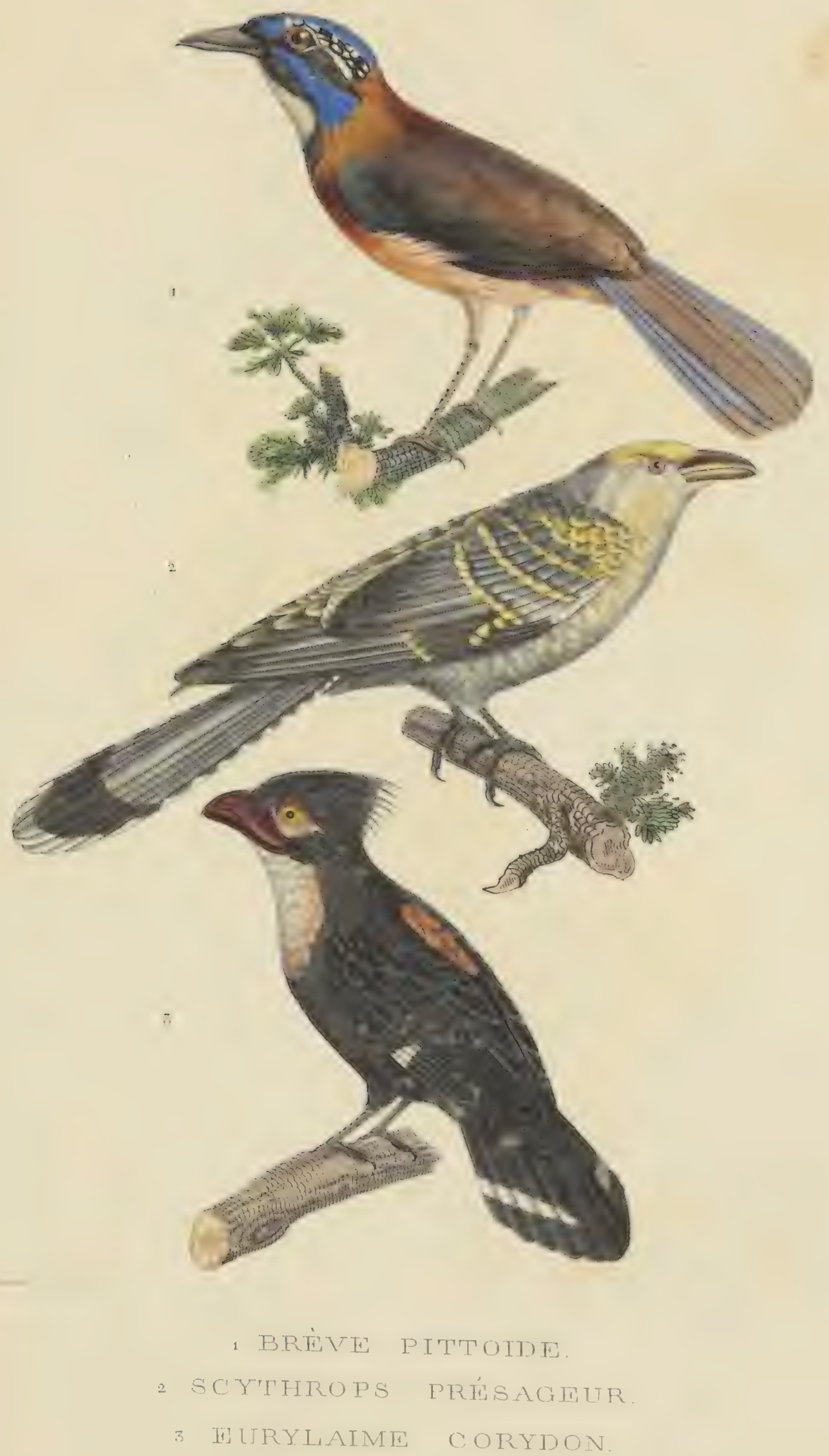



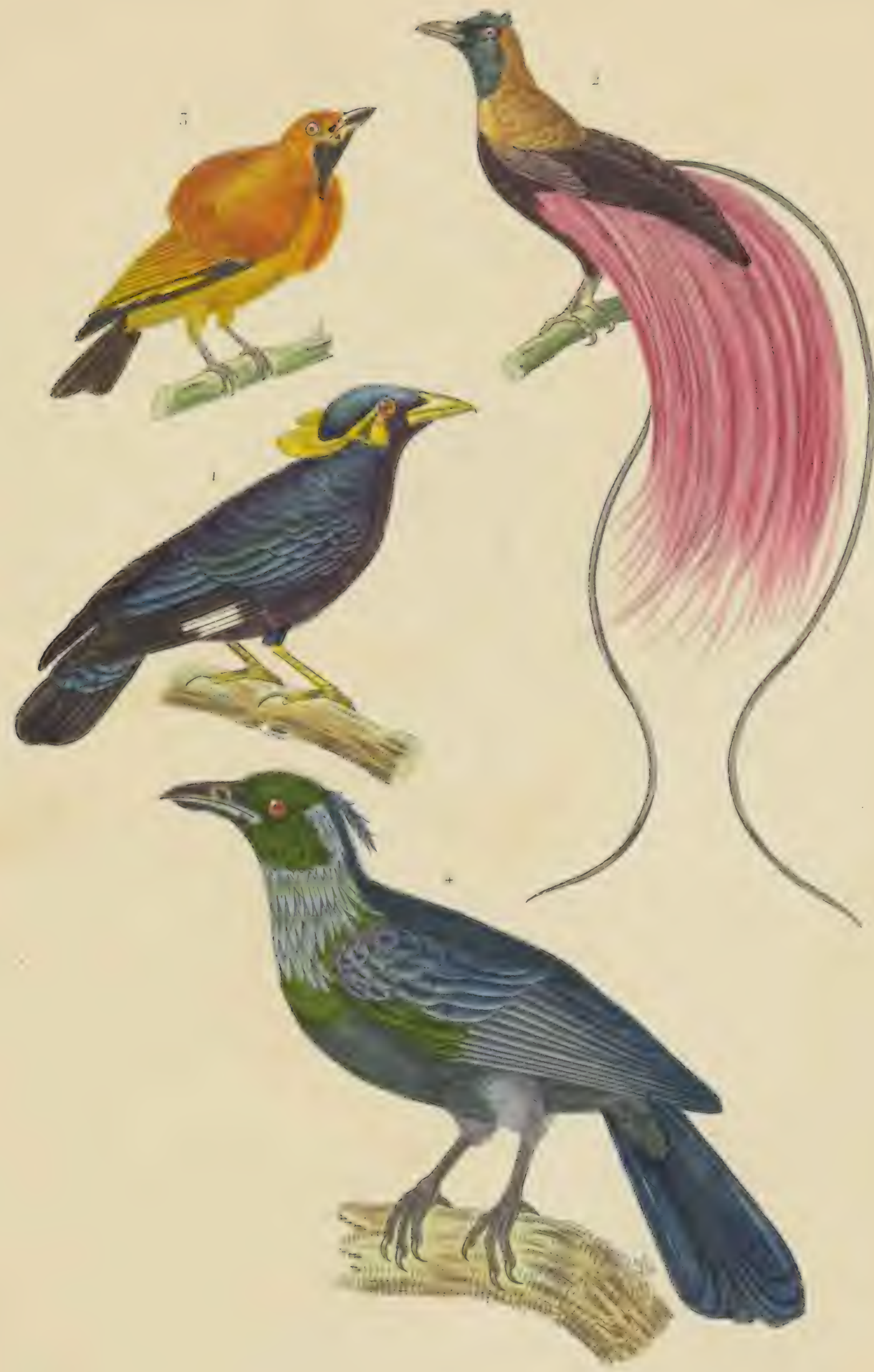

1. MATASTL de Java | 3. SERTCULE orangé,

2.PARADISTHR rouge. I +PHONYGAMT de Kerandren. 


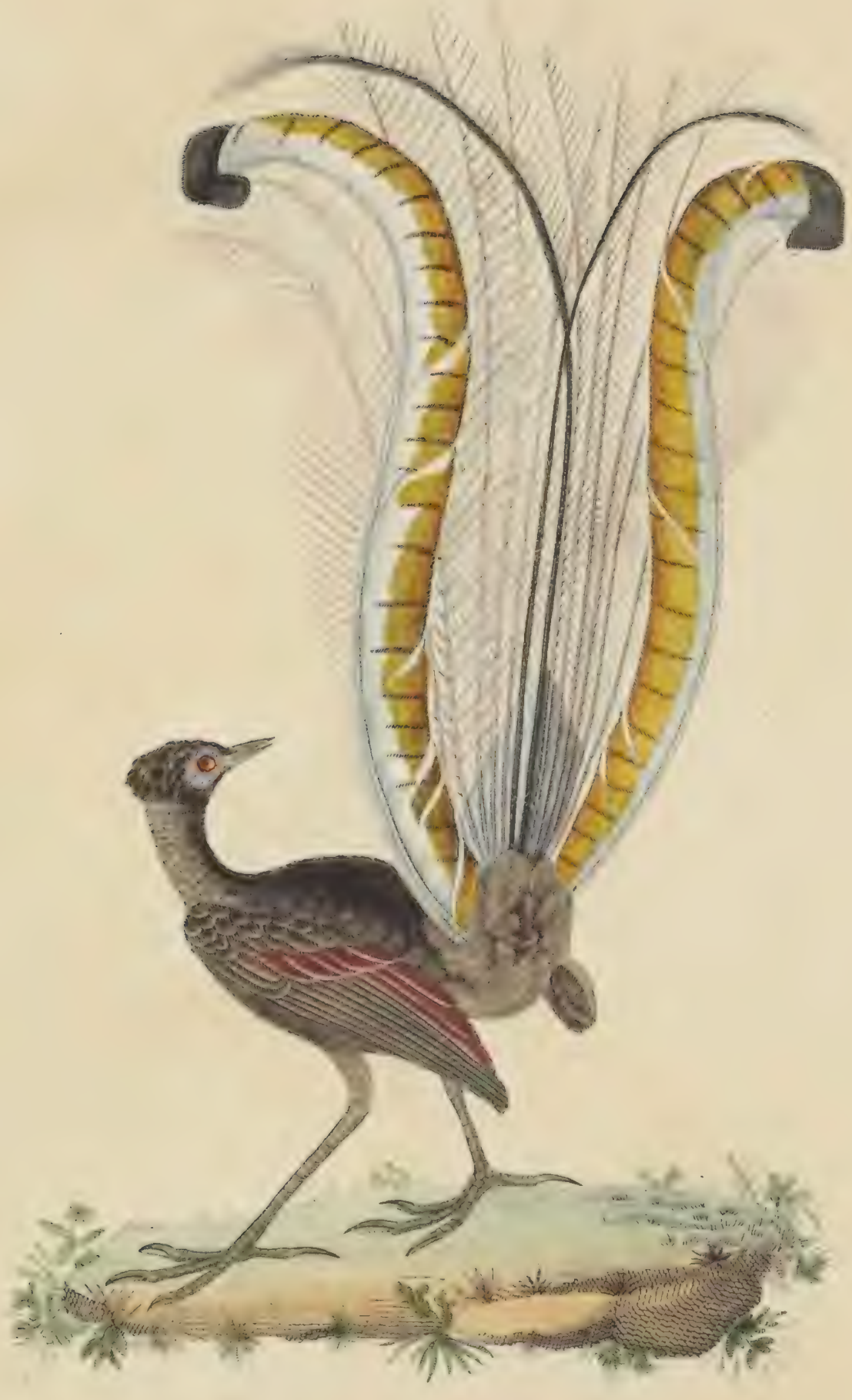

AYLAETUTRA ZIY IITA。

MENURE LTTE. 


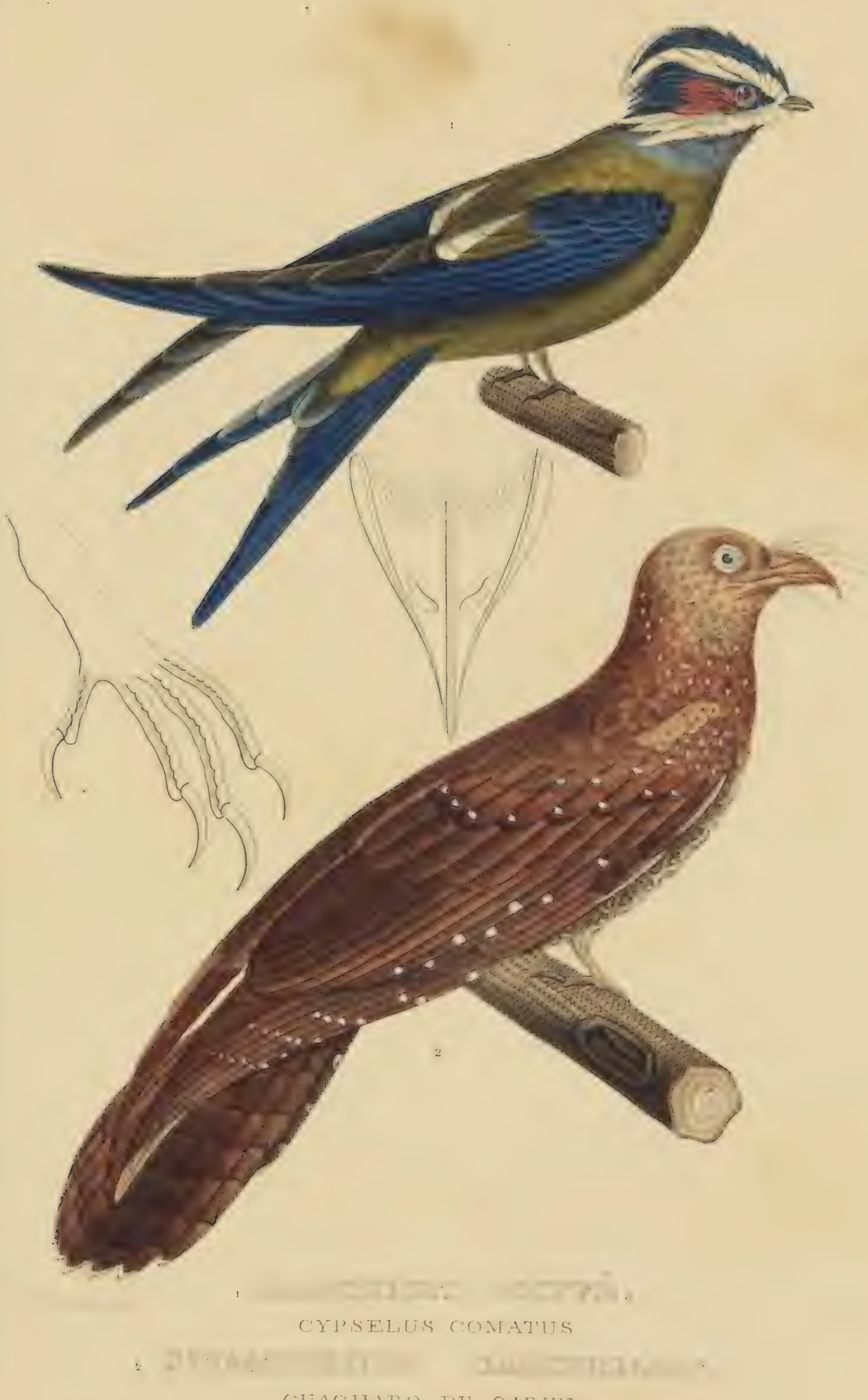

GUACHARO DE CARIPF 


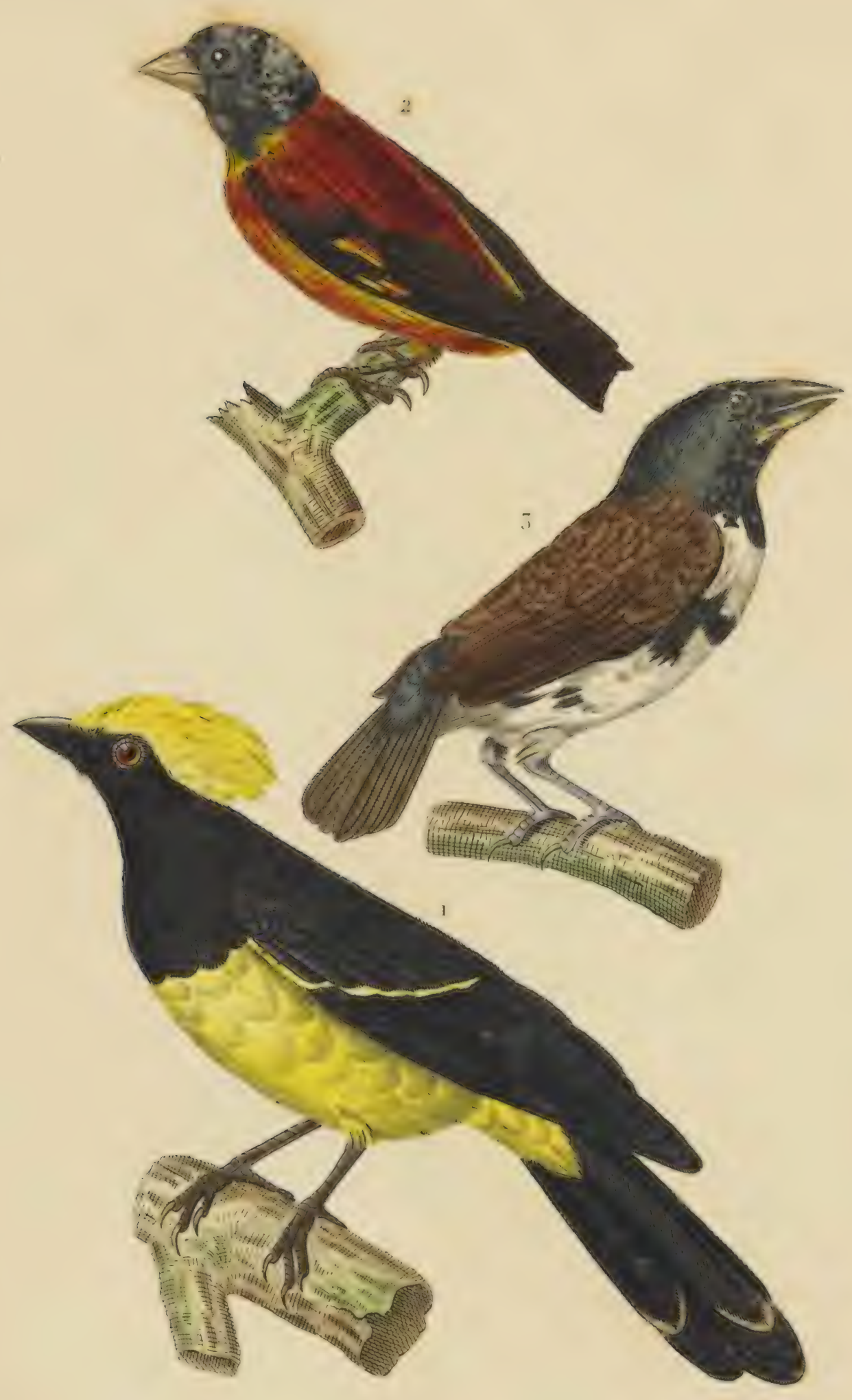

1. P.SRUS FTATO-CRISTATUS. Mésanoe a huppe jaune

2. HRINCIIALA CLHBL. Gros-bee de cuba.

5. PLOCHUS FRINGIIJOID FS. Tisserin fringillöide. 

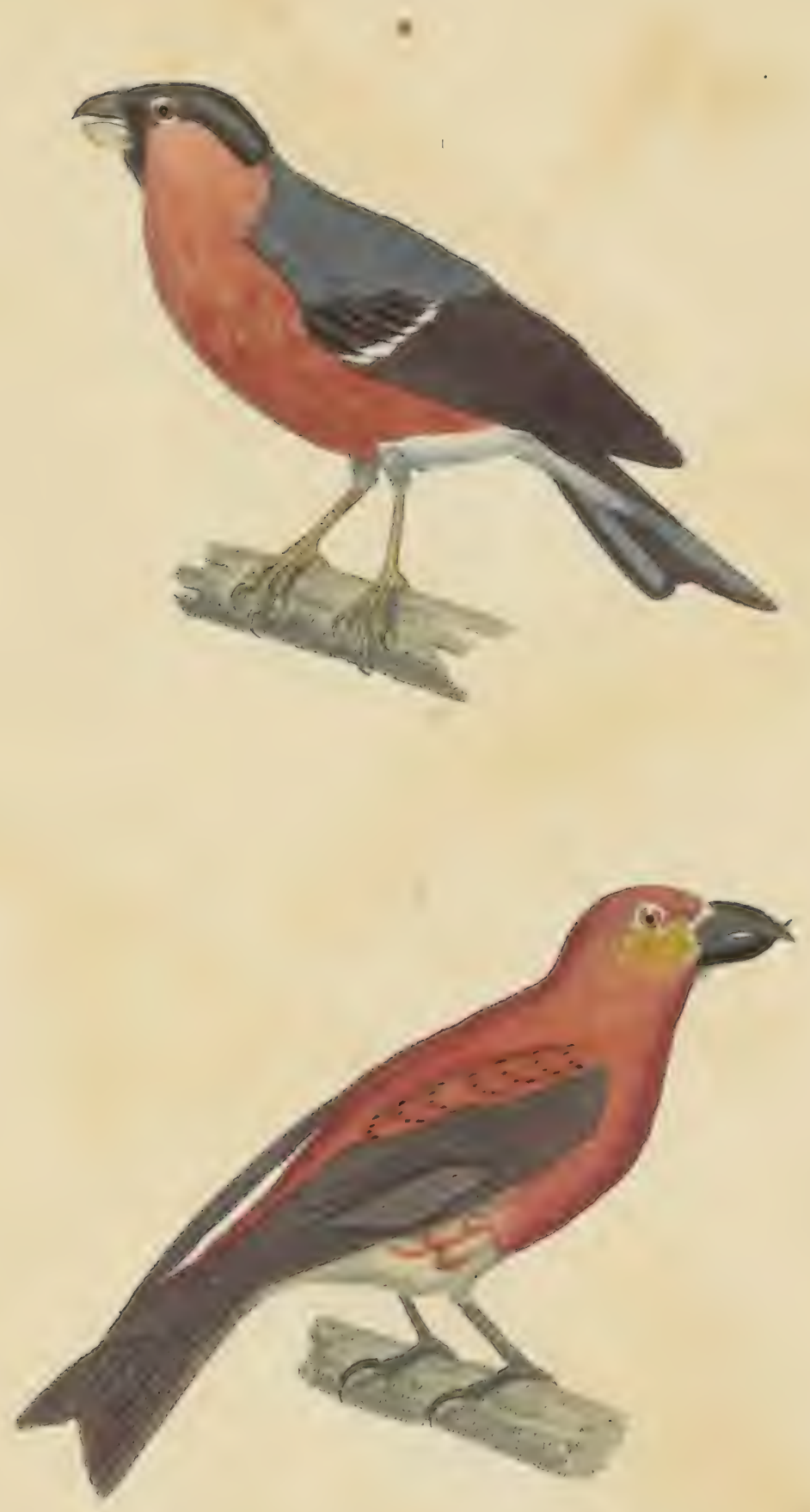

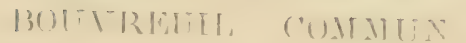

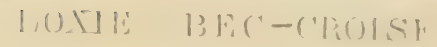




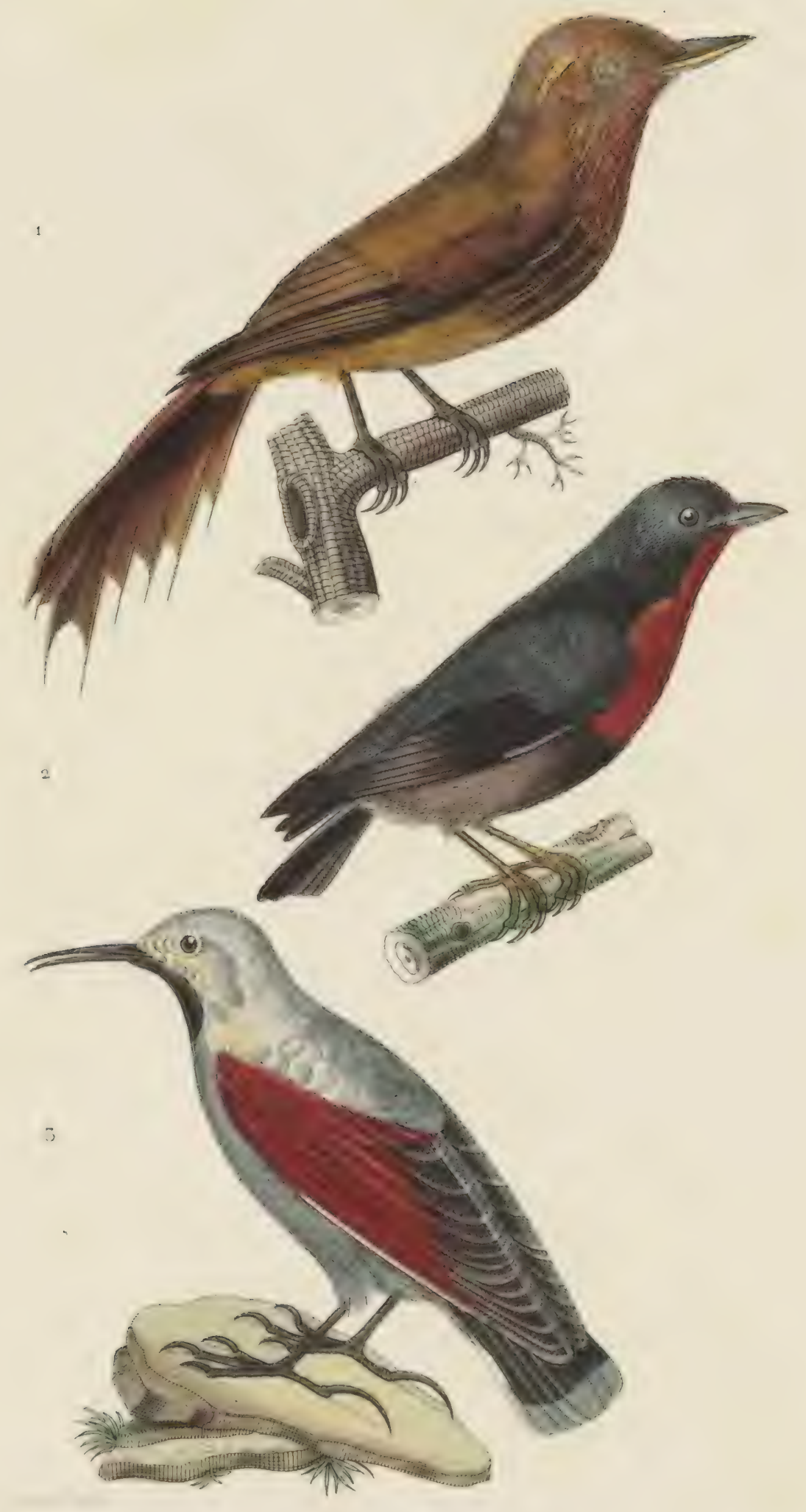

1 PICUCULE A BEC EN $11 N$

2 DICEE A PLASTRON.

- TIOHODROME: FCHEIFTTH 


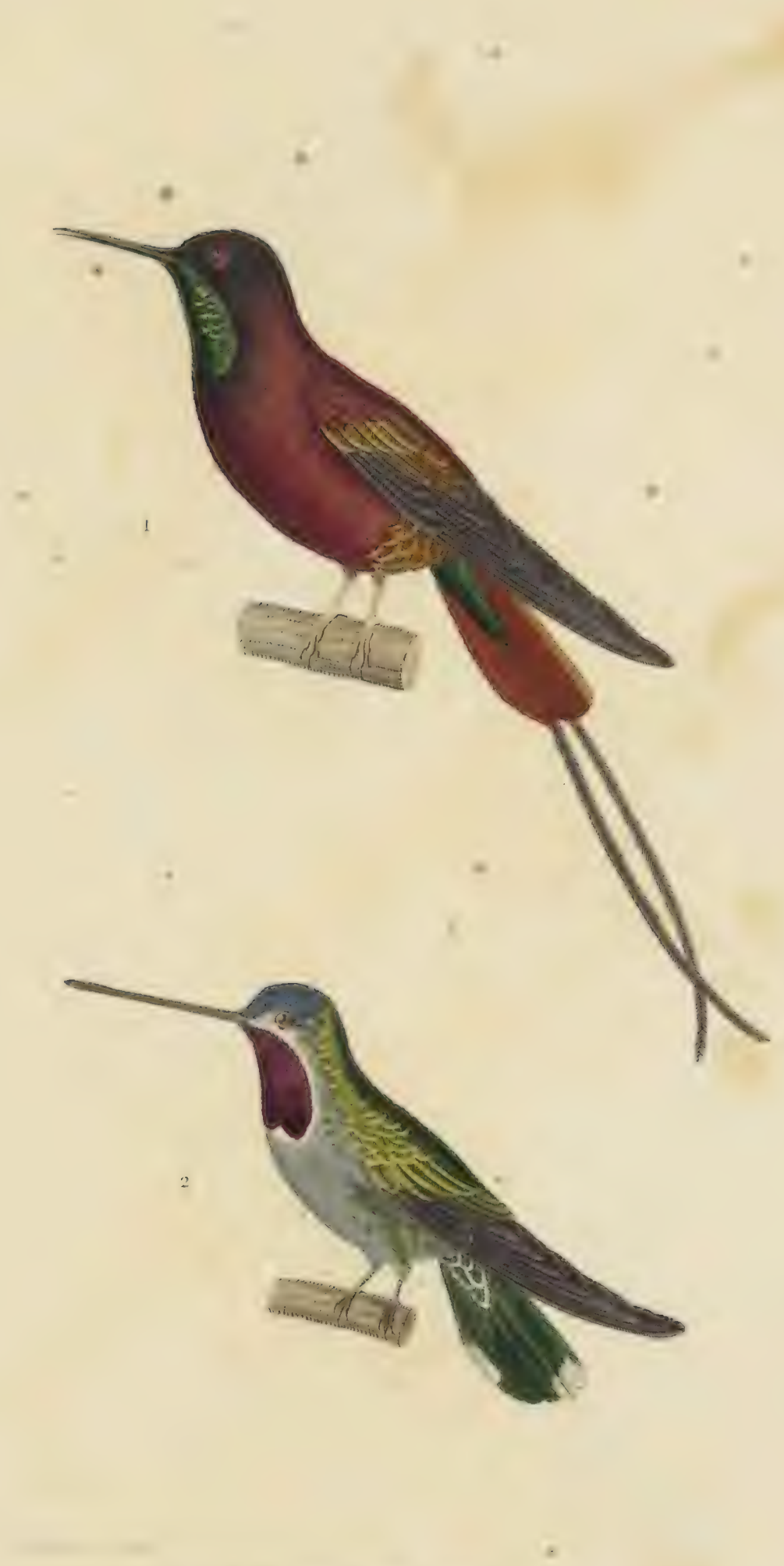

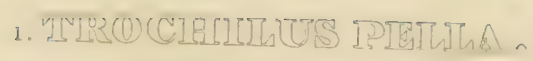

COLIBRI TOPAZF.

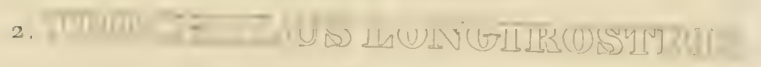

COLIBRI A LONG BEC. 


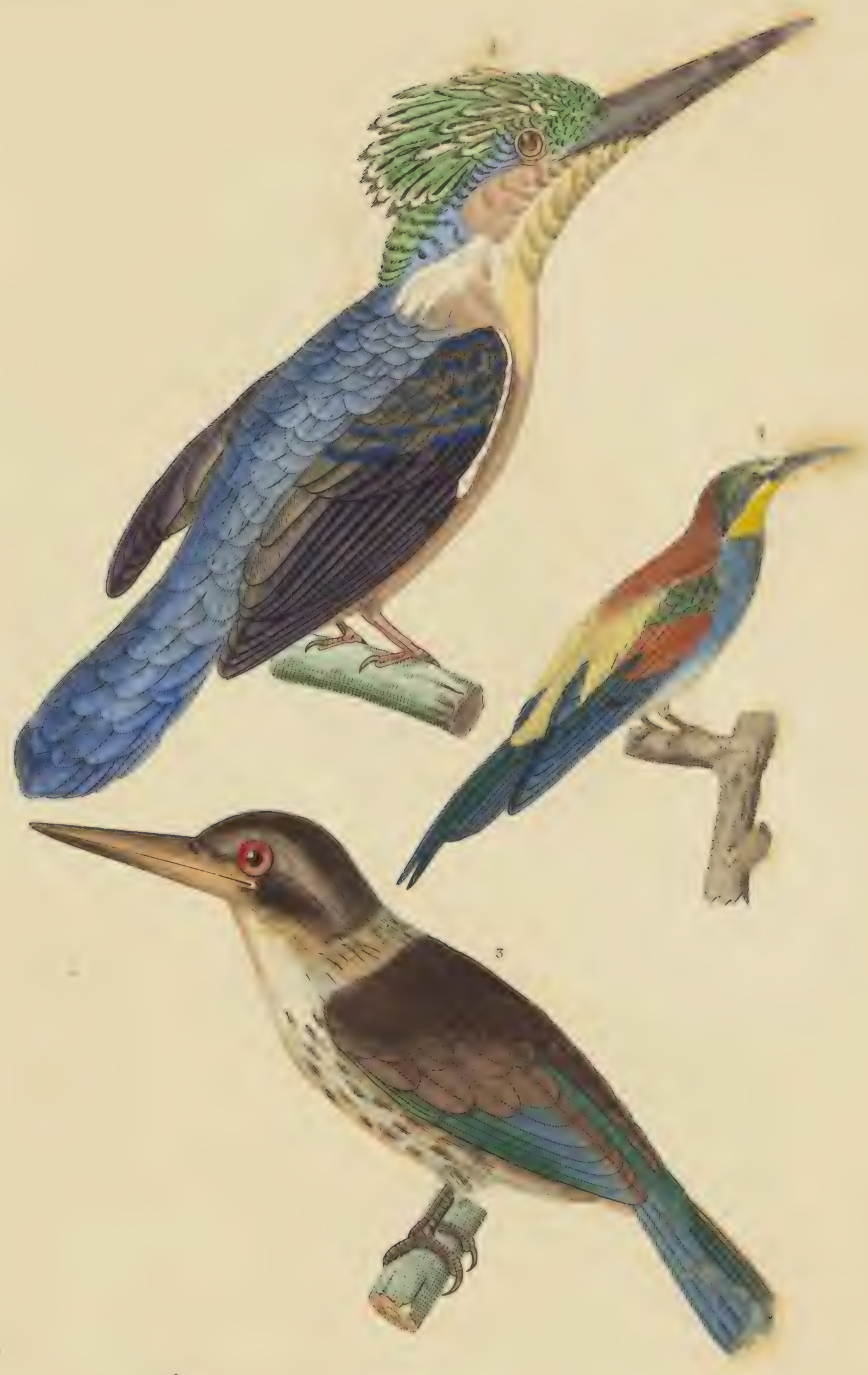

1 GUEPIER COMMUN. Merops apiaster.

2 MARTIN-PECHEUR VINTSIOIDE. Alcedo vintsioides.

3 MARTIN-CHASSEUR A COIFFE BRUNE. Dacelo fuscicapilla. 


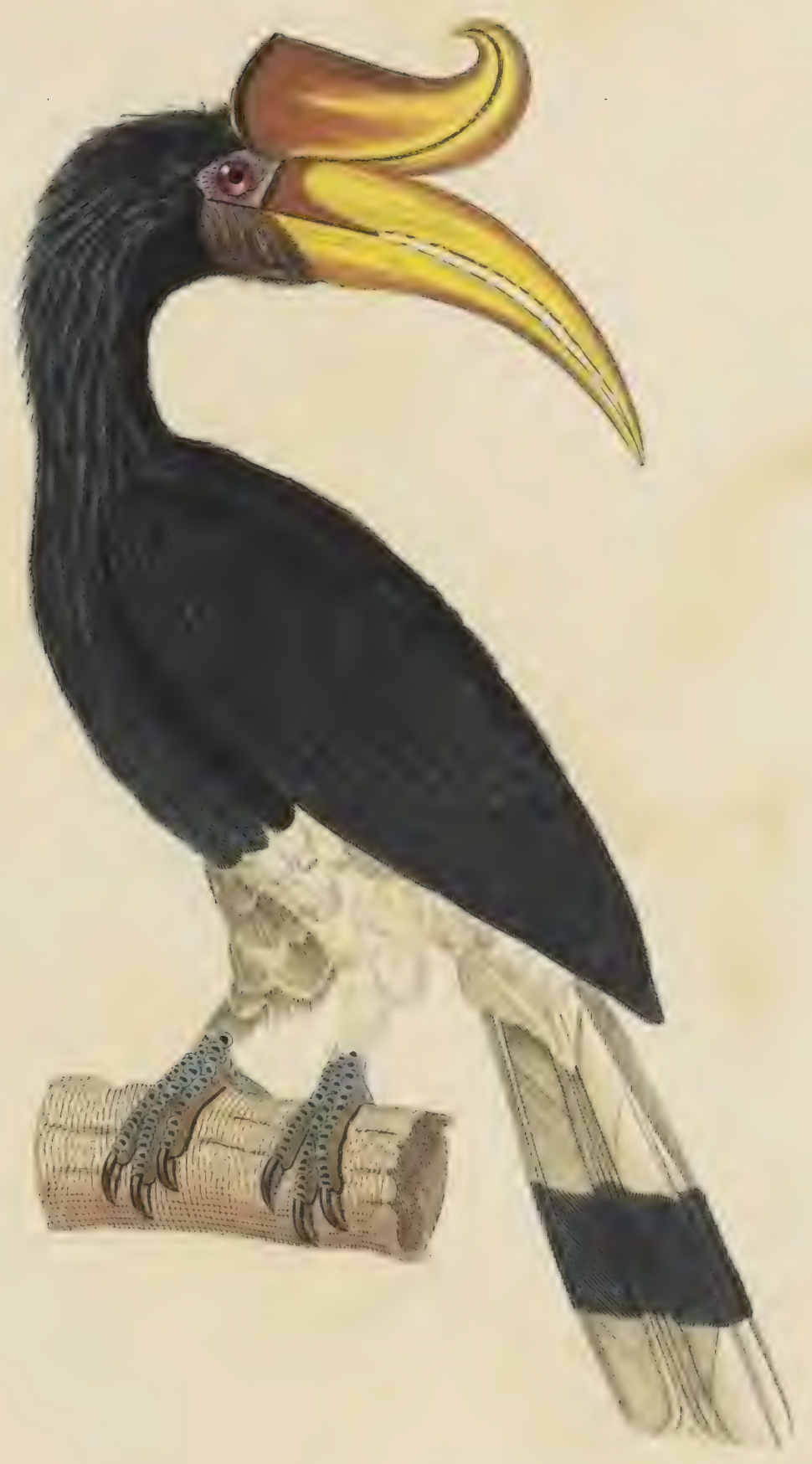

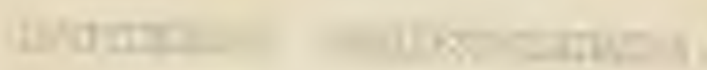

CALAO RHINOCFROS 


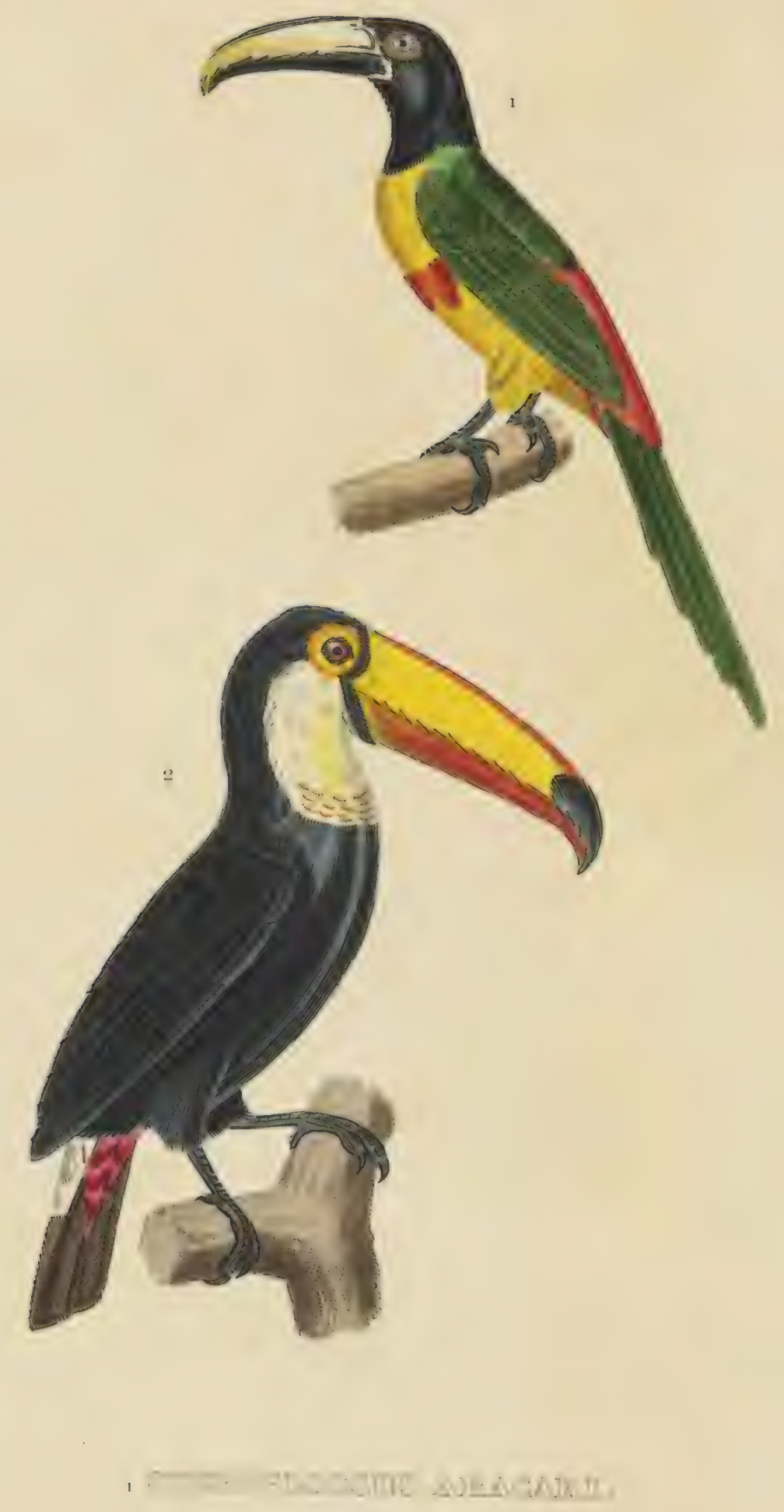

ARACARI GRIGRI.

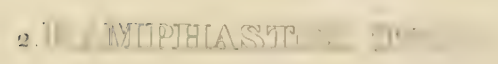

TOUCAN TOCO 


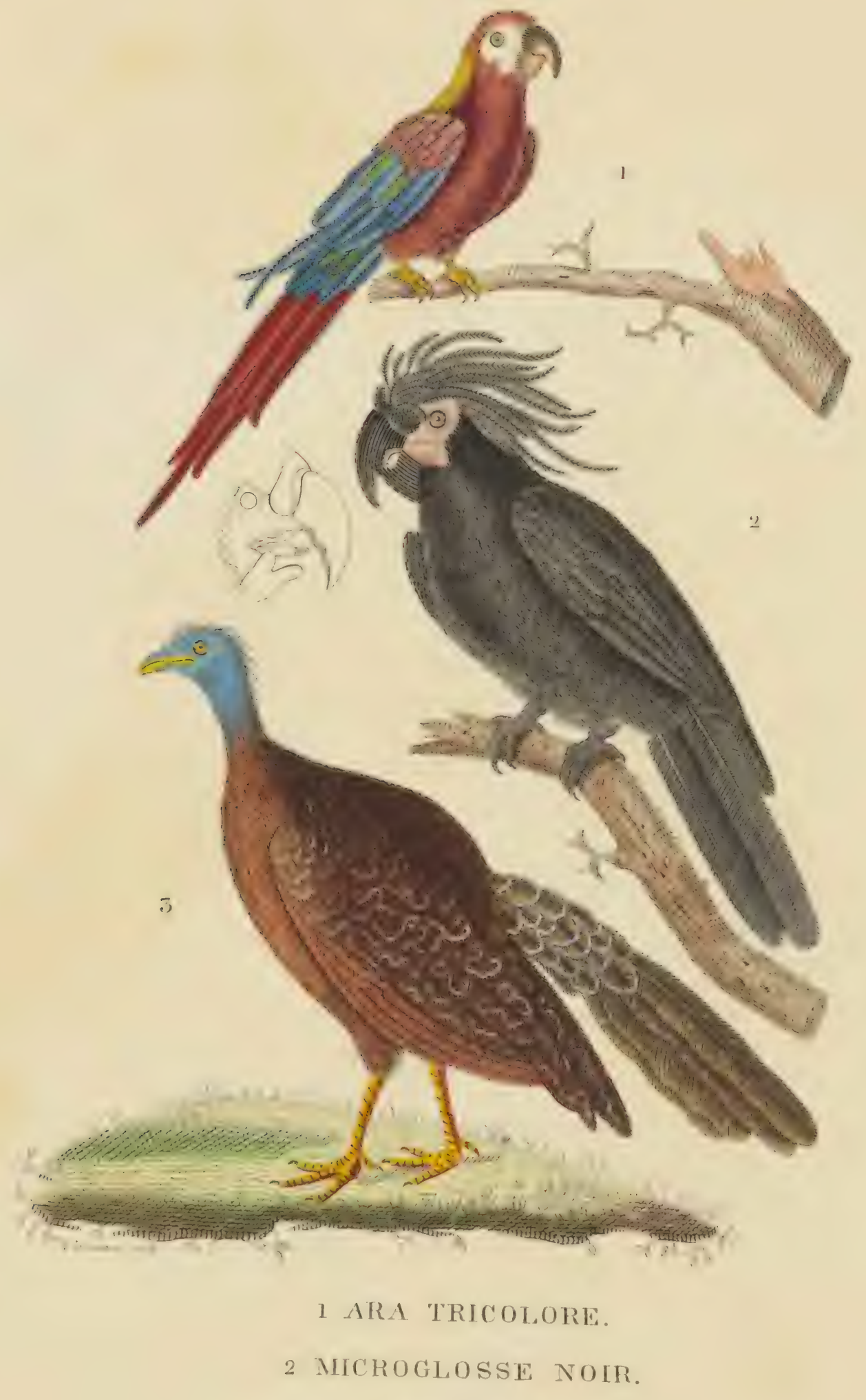

5 ARGUS LUEN. FEMELLE. 


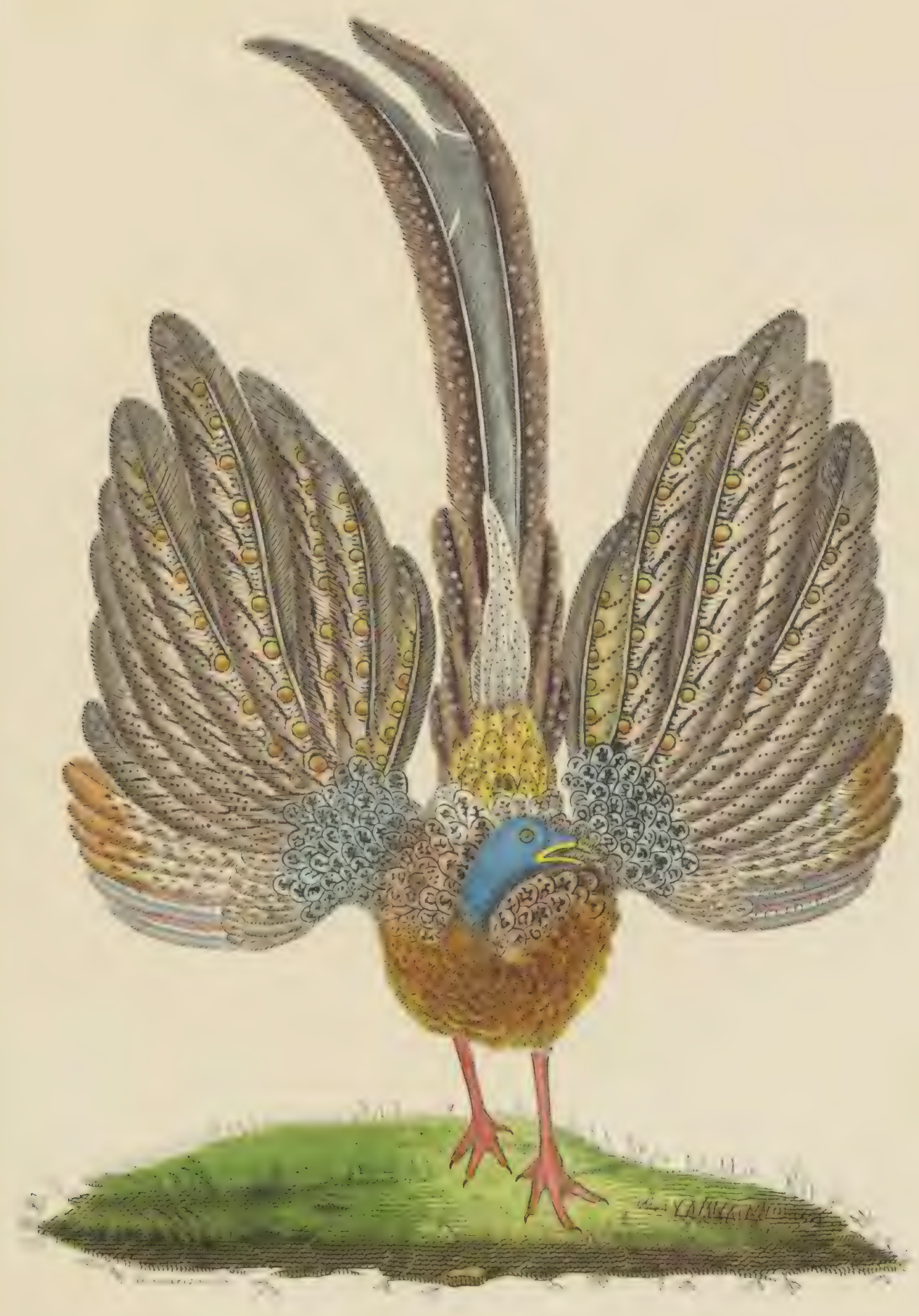

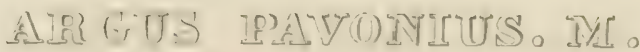

ARGUS IUUEN. MAIE. 


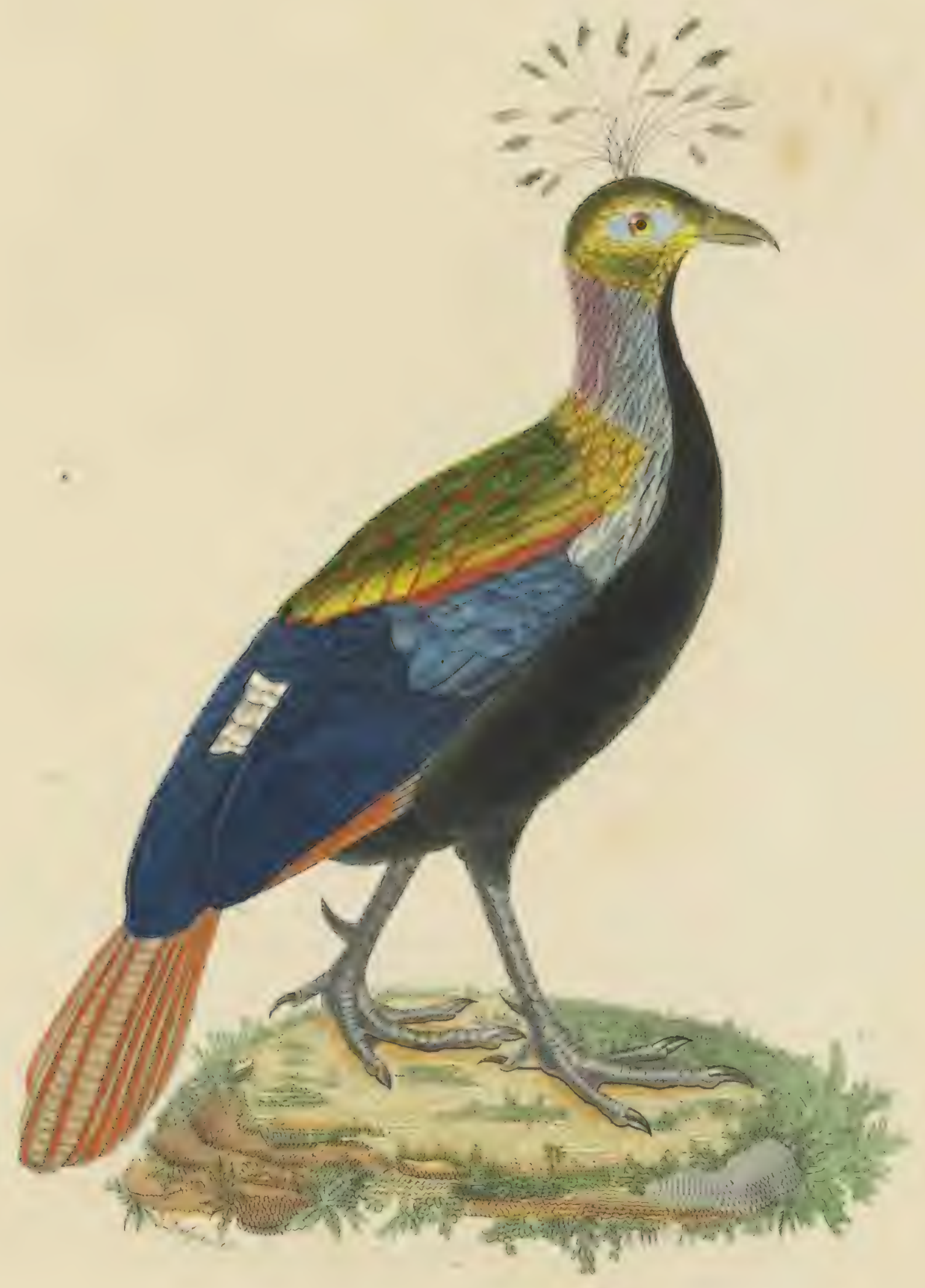

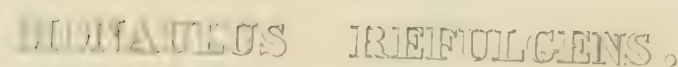

MONAIT RESPJENDISSANT. 


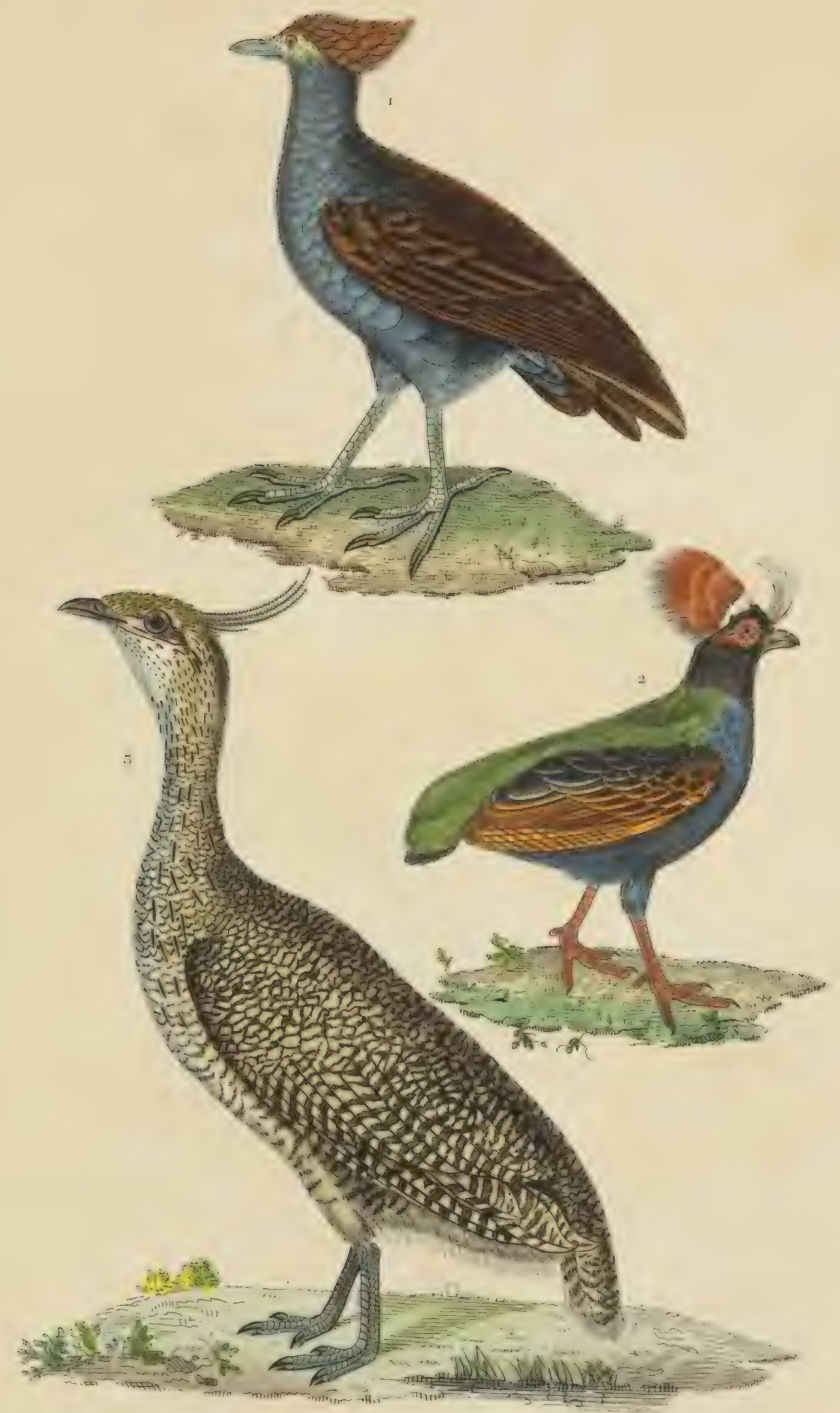

s ME'GAPODE HUPPÈ. Meganodius cristatus.

2 TURNIX A BANDEAU NOIR. Hemipodius migrifrons.

3 EUDROMIE ĹLÉ GANTE. Eudromia elegans.

$$
\text { Desmares sculp. }
$$




$$
\frac{1}{2}
$$




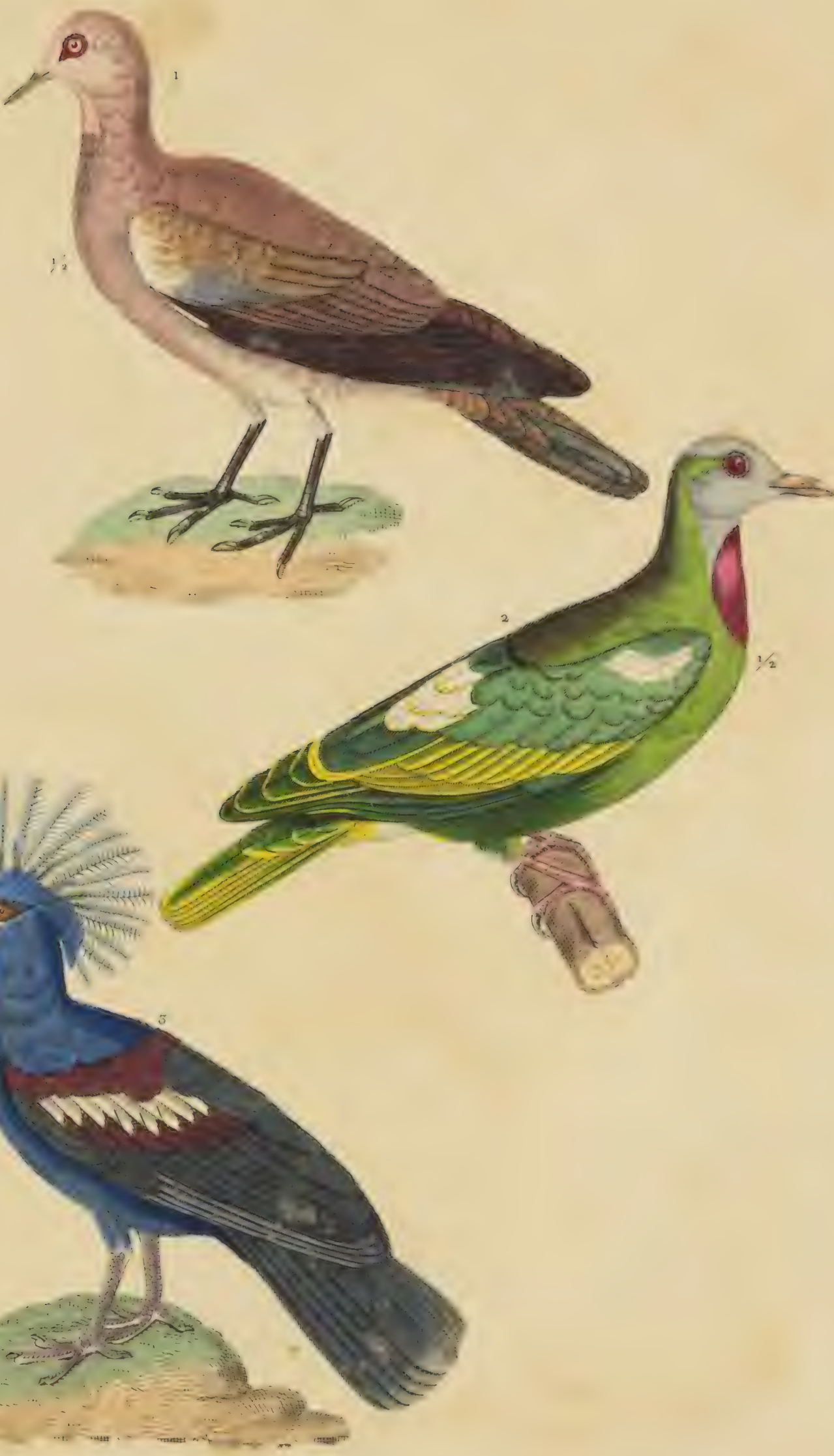

1. PIGEON-COLOMBE de Bolivie.

2. PIGEON-COLOMBAR à gorge poruprée

3. PIGEON-LOPIYYRE Gouna.

Demarnes Scuint 


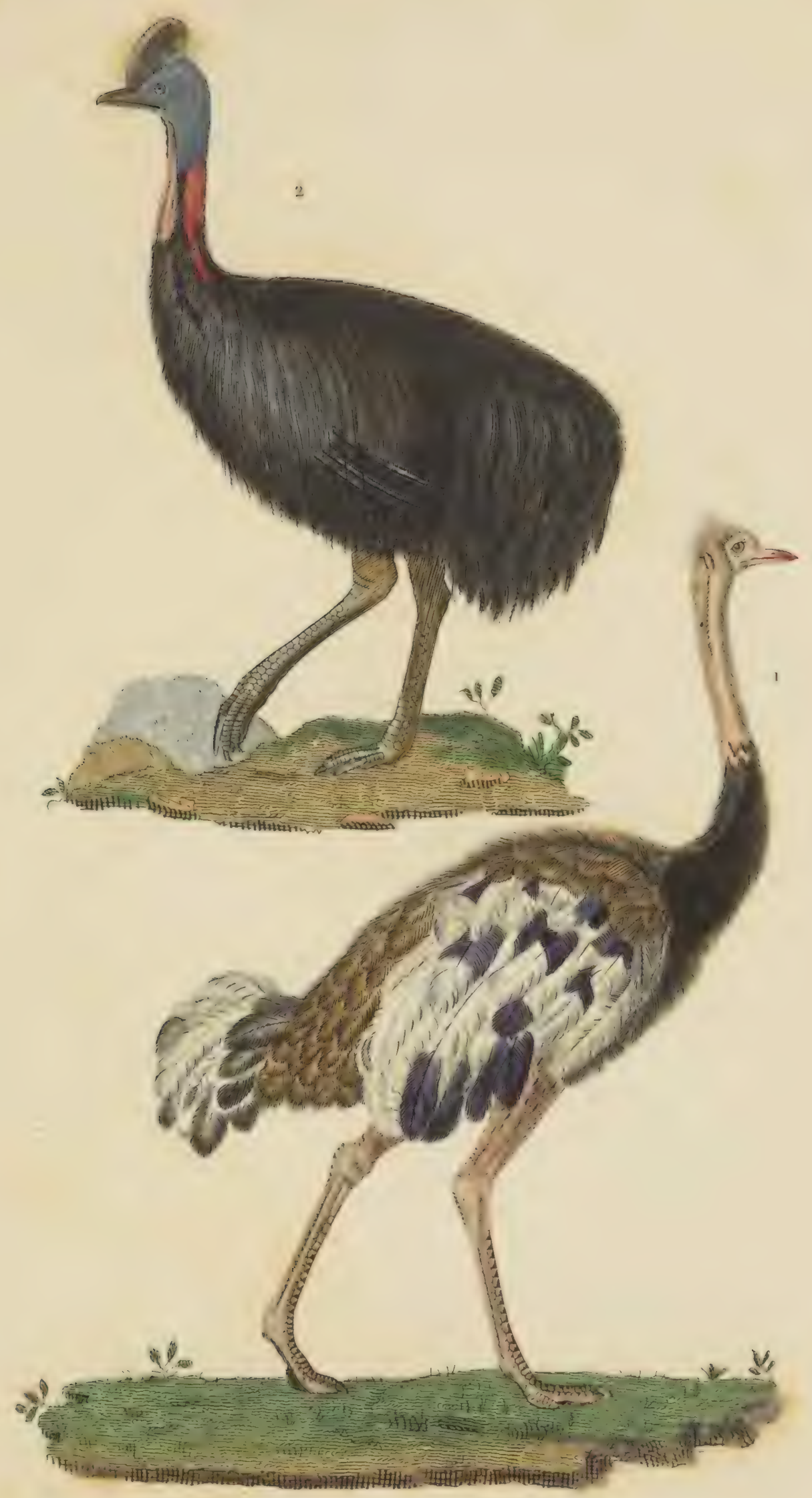

1. AU'I'RUCHE: struthio camelus.

2. CASOAR casuarizs oaleatus. 

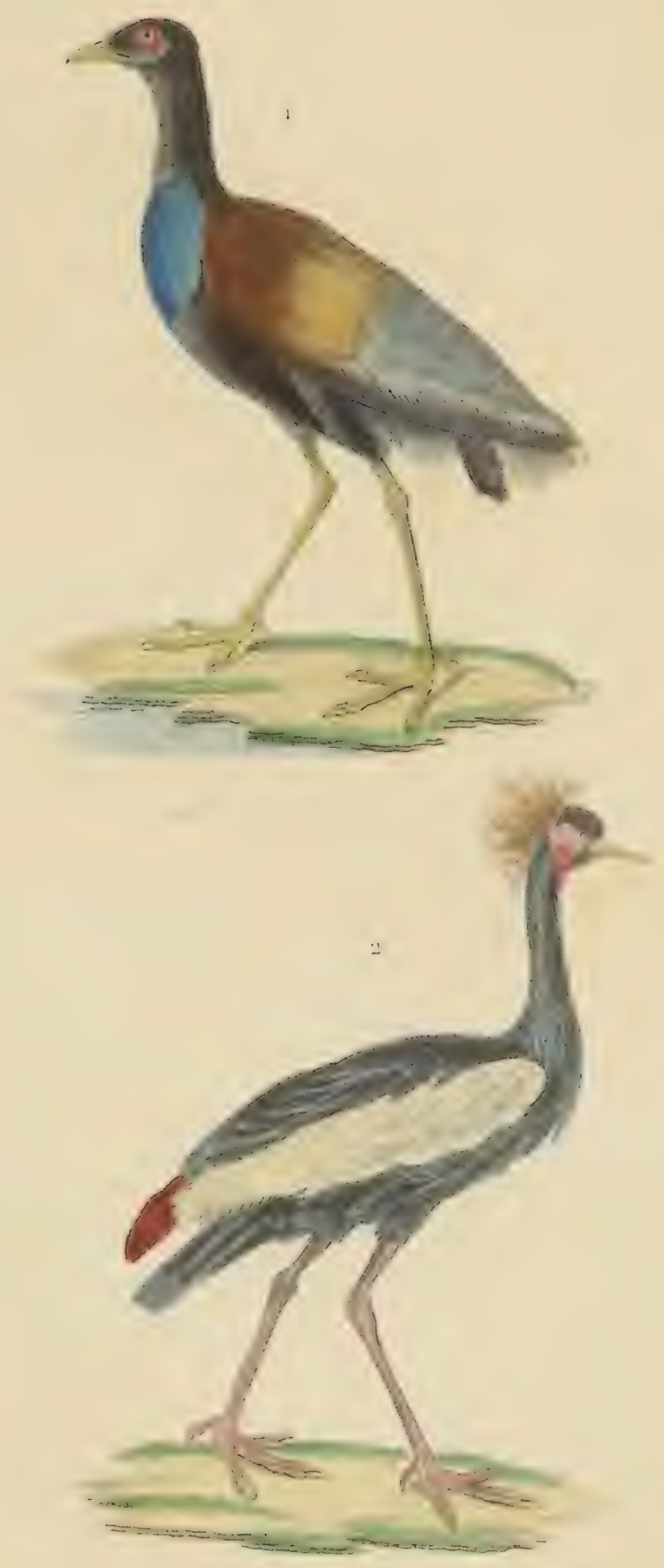

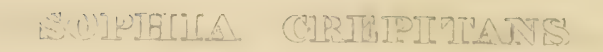

AGAMI TROMPITTK.

2. ATRTDIEA IPAVONINA.

GRLE COURONNFE. 

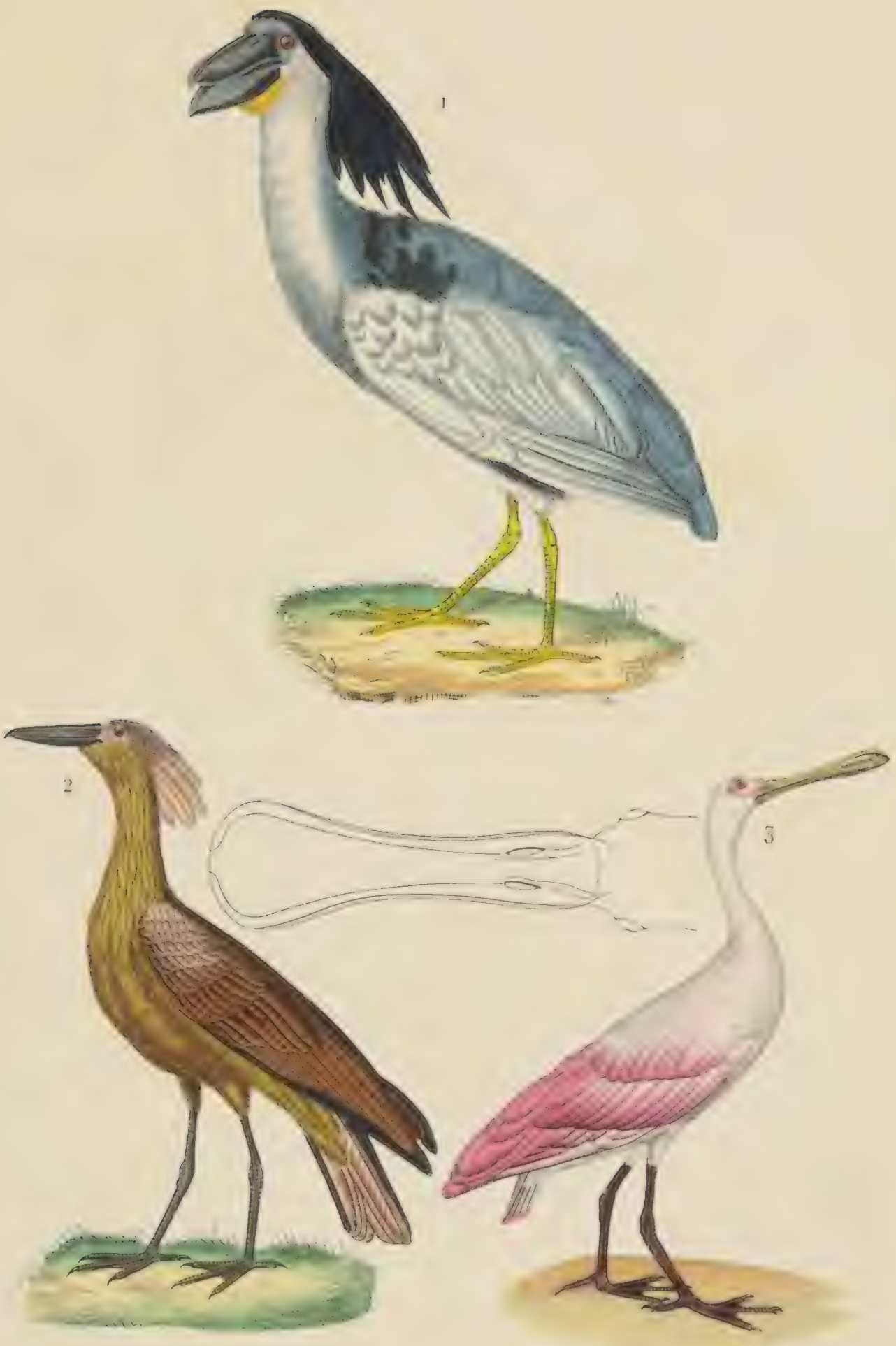

1. SAVACOU COCHLEARIA. Cancroma cochlearia .

2. OMBRETTE DU SENF, GAT. Scopus umbretta.

3. SPATULE ROSE. Platalea ajayja 

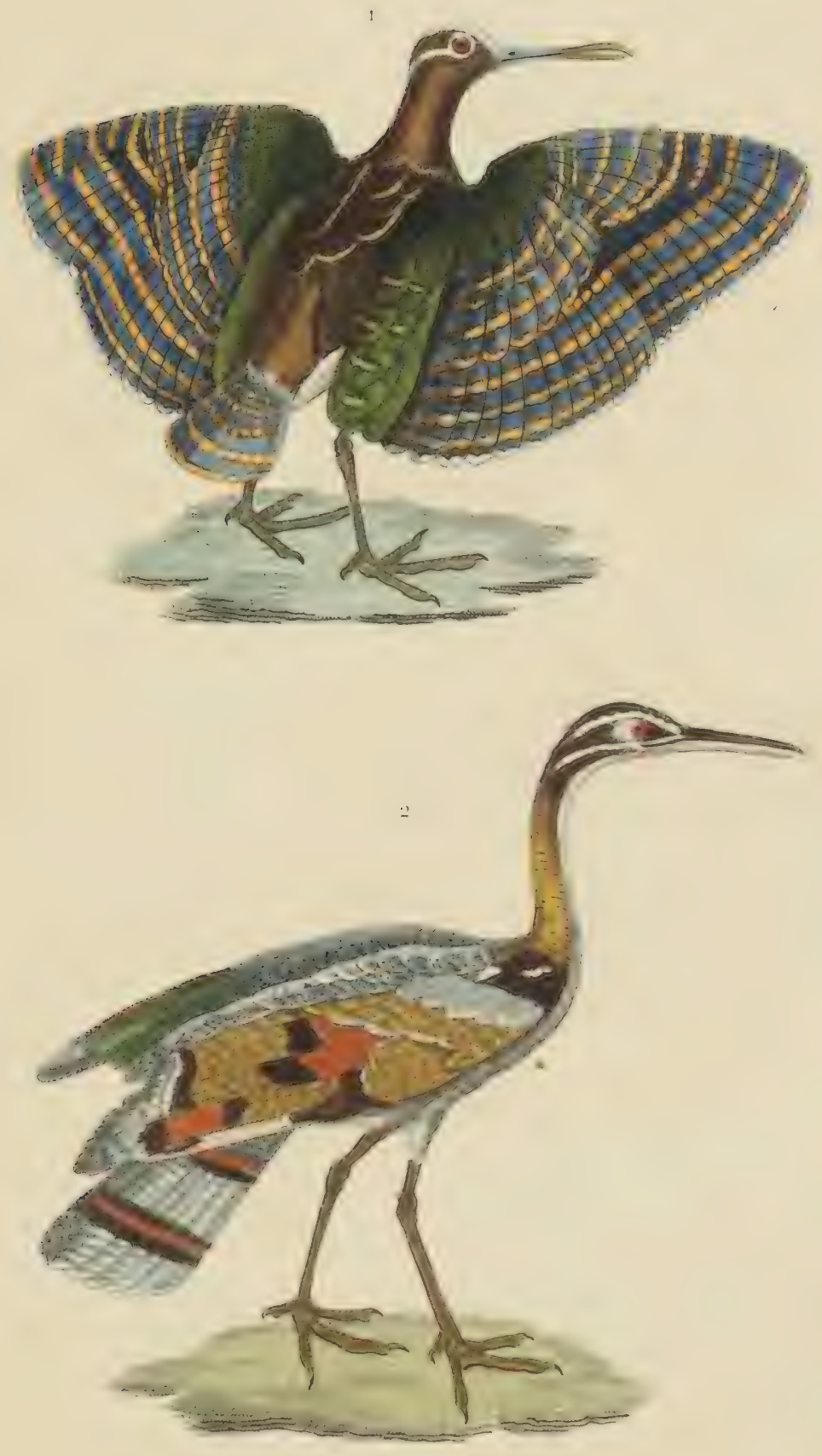

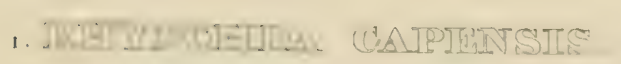
RIYACHĹE du Cap ou de Madagascar.

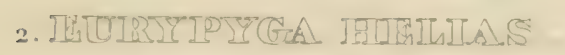
CAURALE PIAIÉNOÏDE. 


$$
1
$$



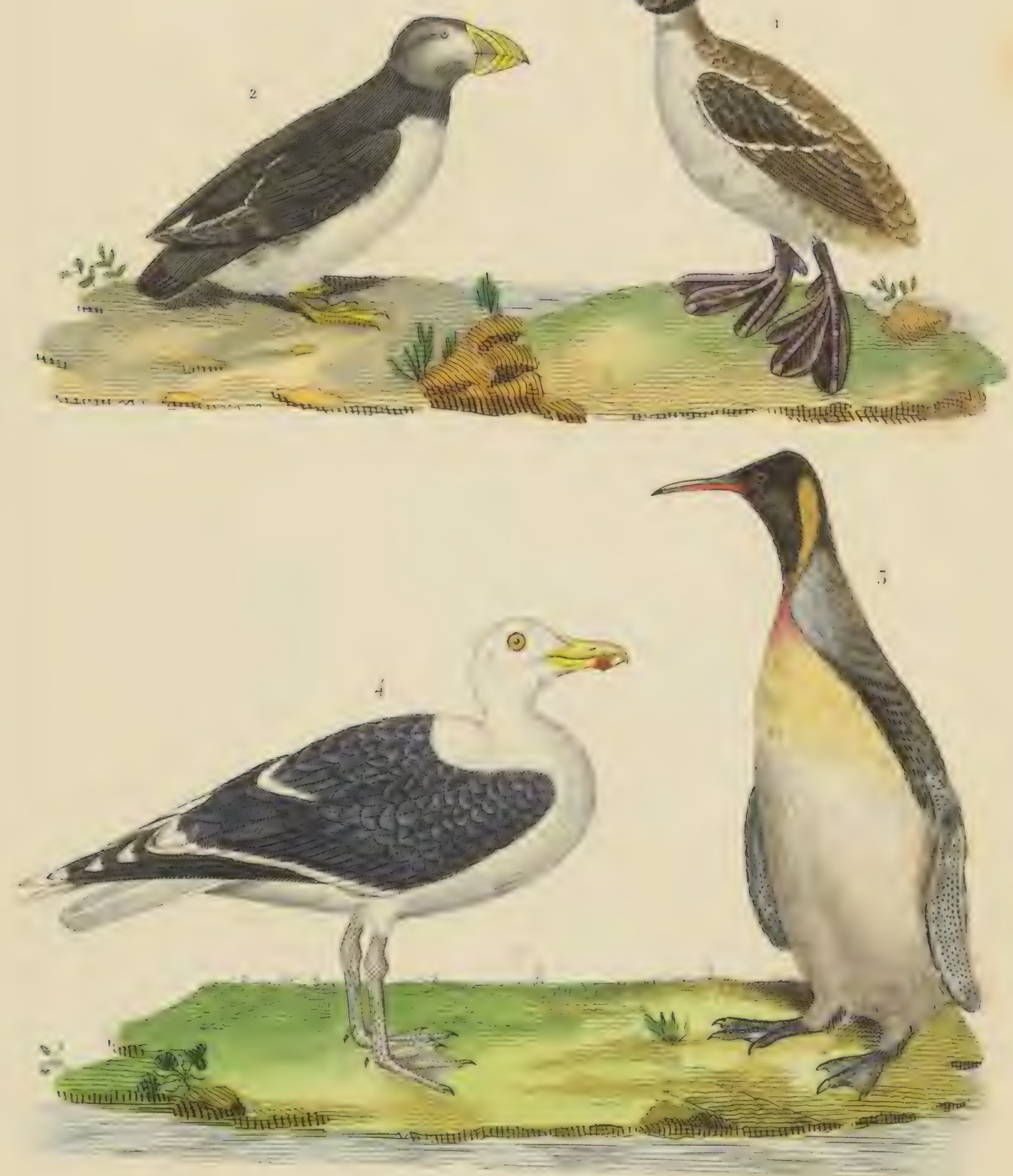

1. PODTCEPS CRISTATUS, grebe huppé.

2. MORHON FRATFRCULA. maciurux moine

3. APTENODYTES PATACHONICA. girand manchot.

4. LARUS MARINUS, monetle à mantean noir. 

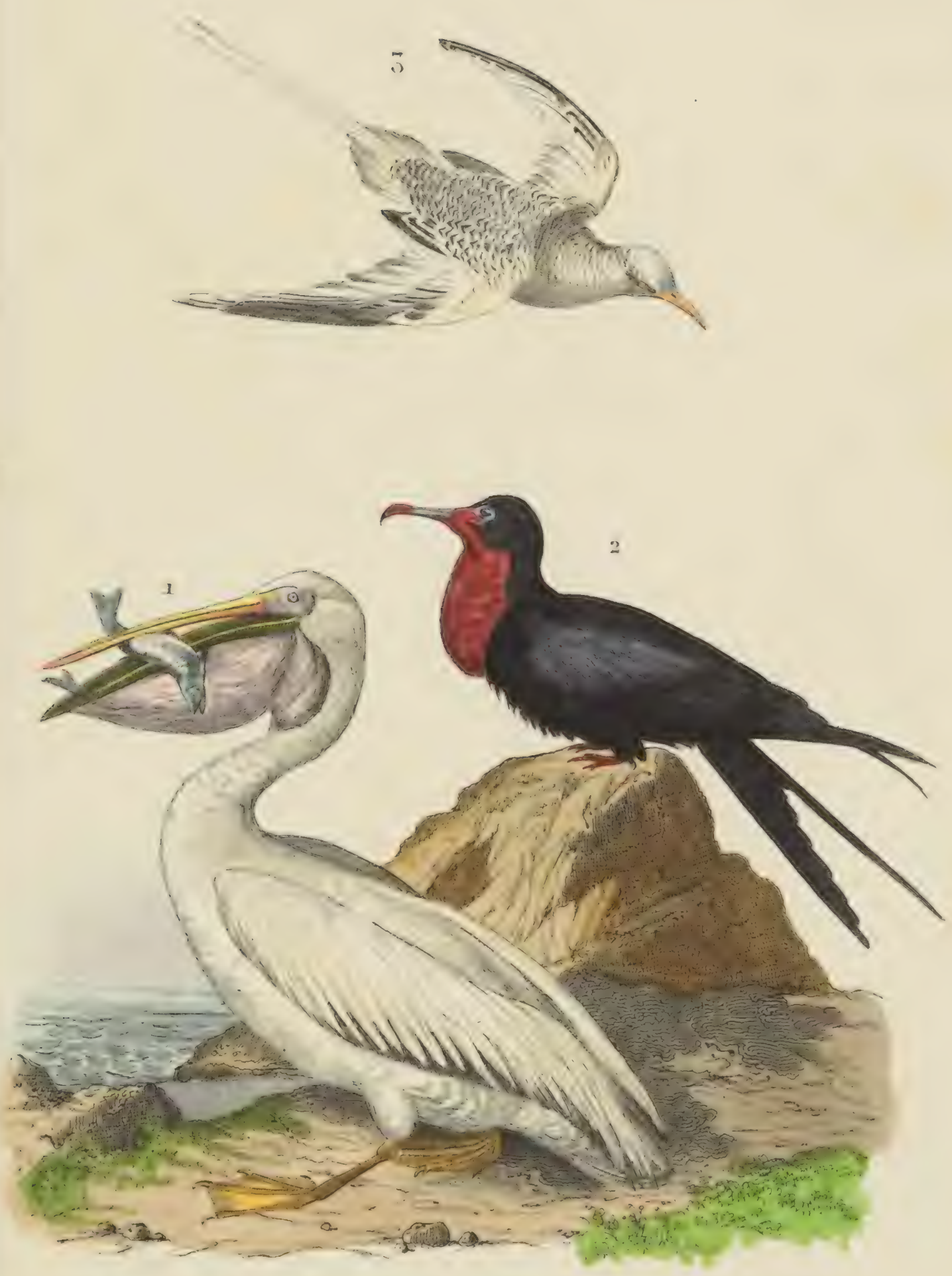

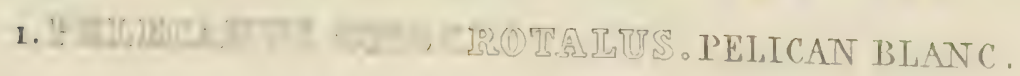

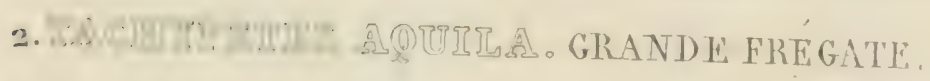

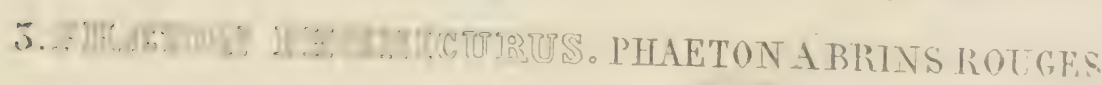




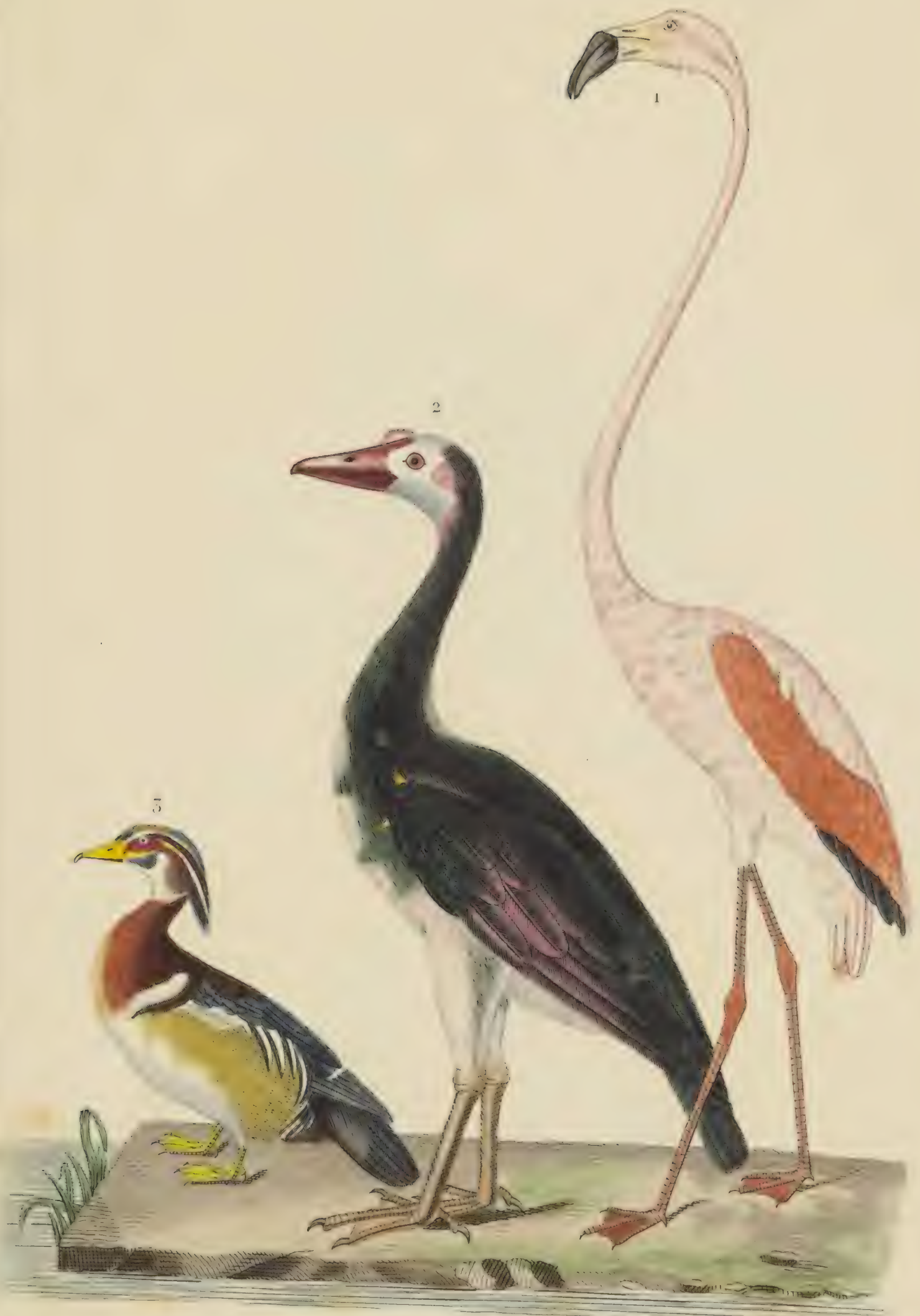

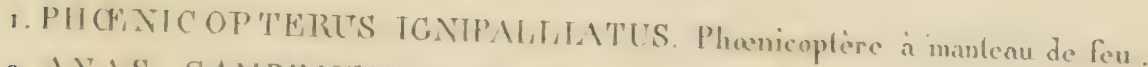

2. $\triangle N$.LS GAMBLNSIS. Oie a couble éperon.

5. ANAS SPONSA. Beau canard huppé. 


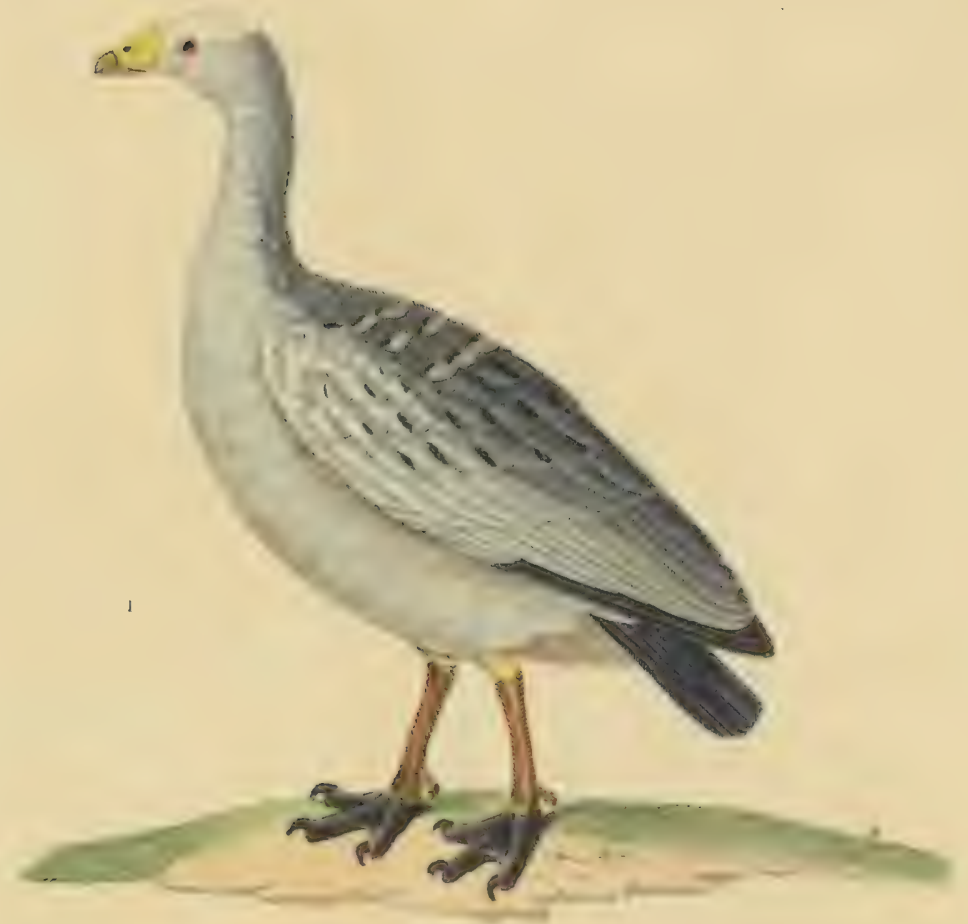

$$
\rightarrow b \rightarrow
$$
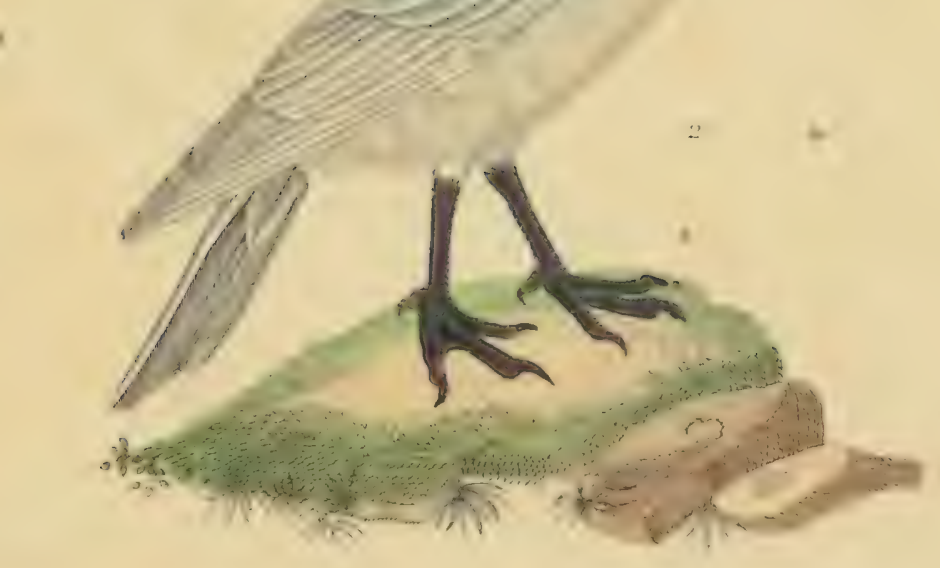

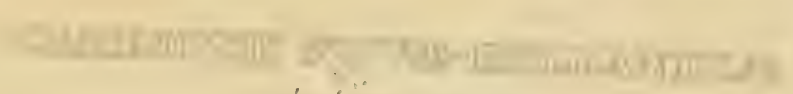

CÉRÉOPS CENDRÉ.

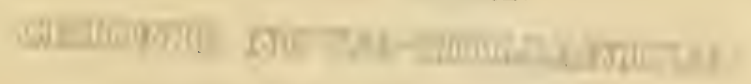

CHIONIS BEC EN FOURRFAU. 

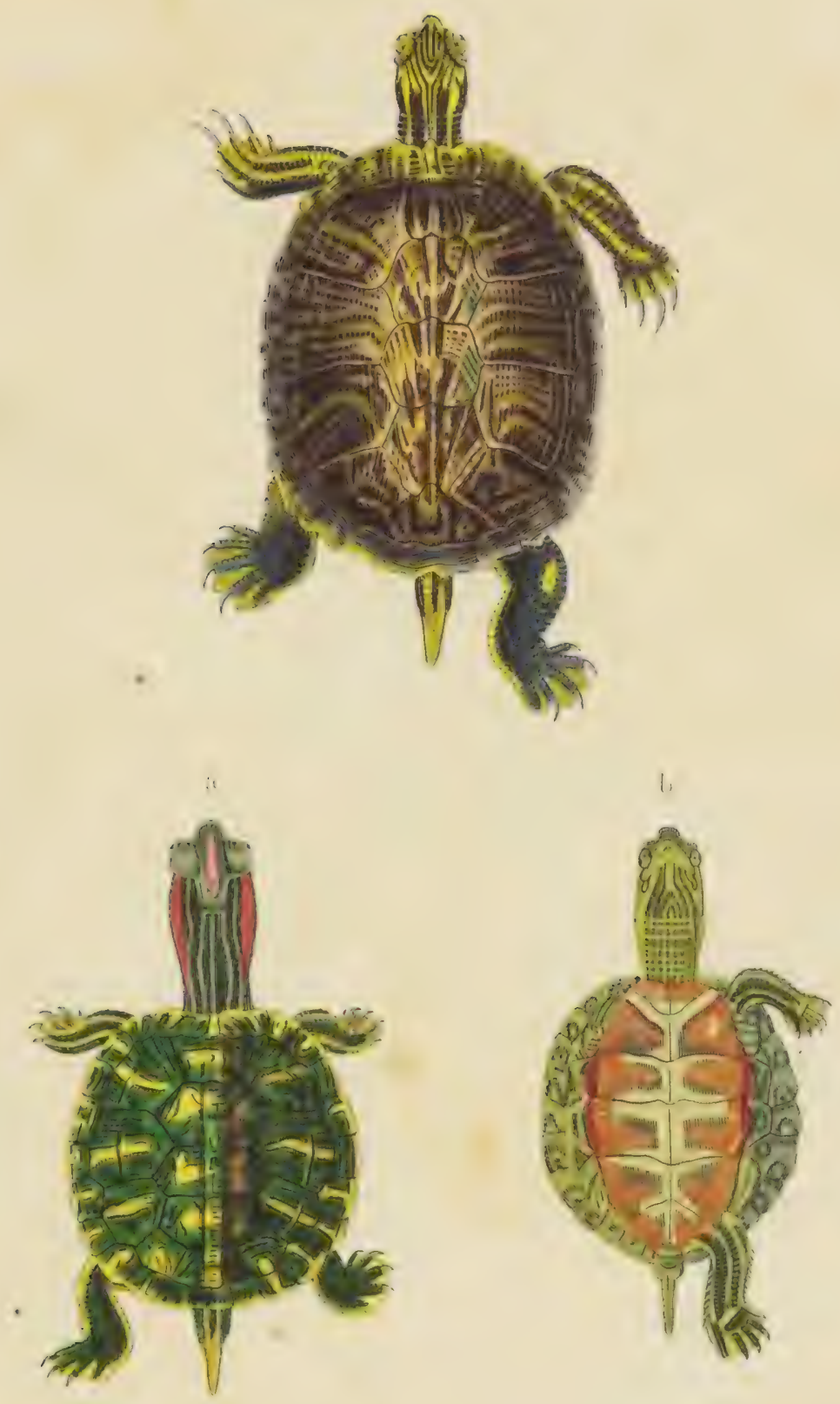

EMYDE. PEINTE 


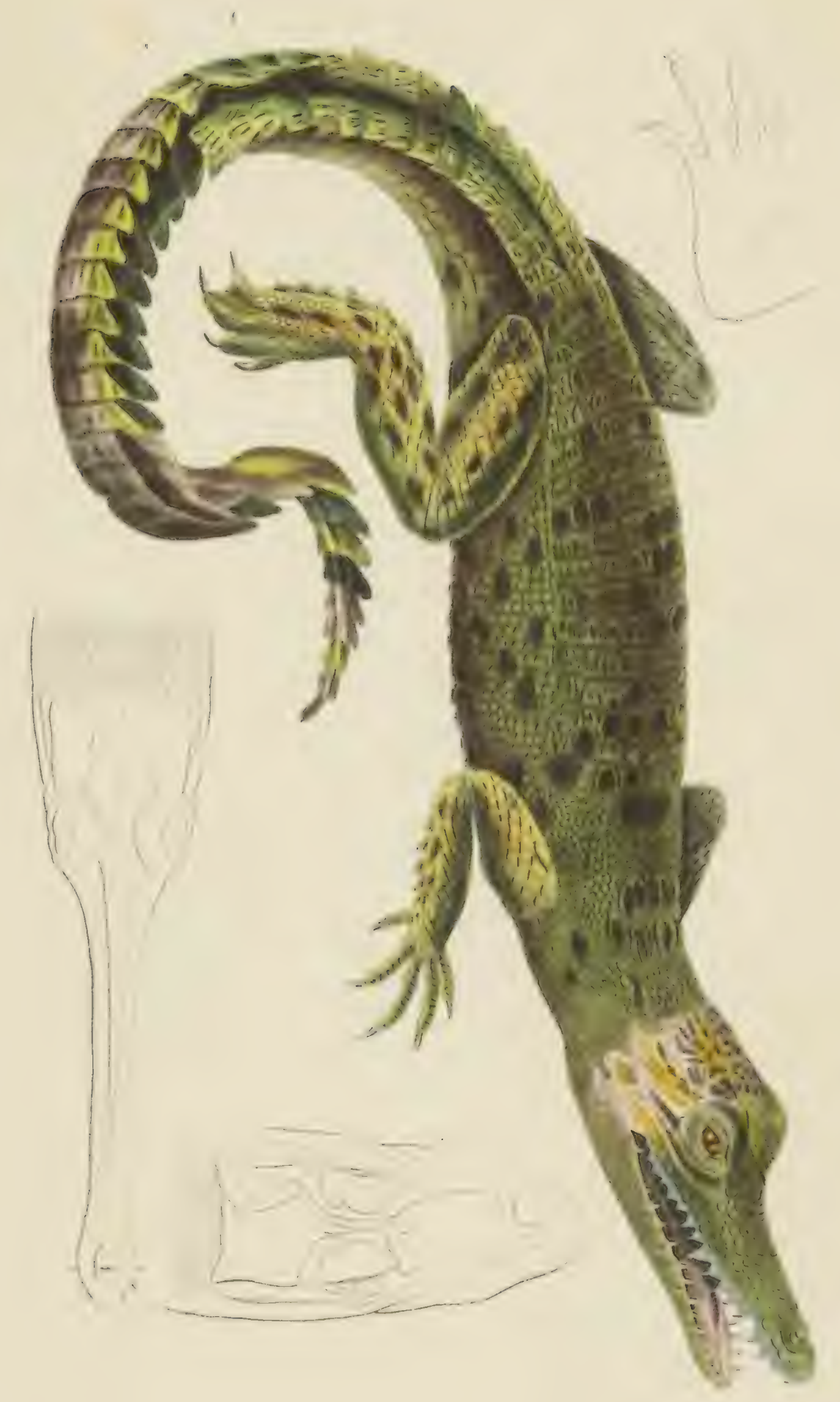

CIIDCODIUSS BIDORCAIOSS.Cuv. CROCODILE A DEUX ARETES. 
3
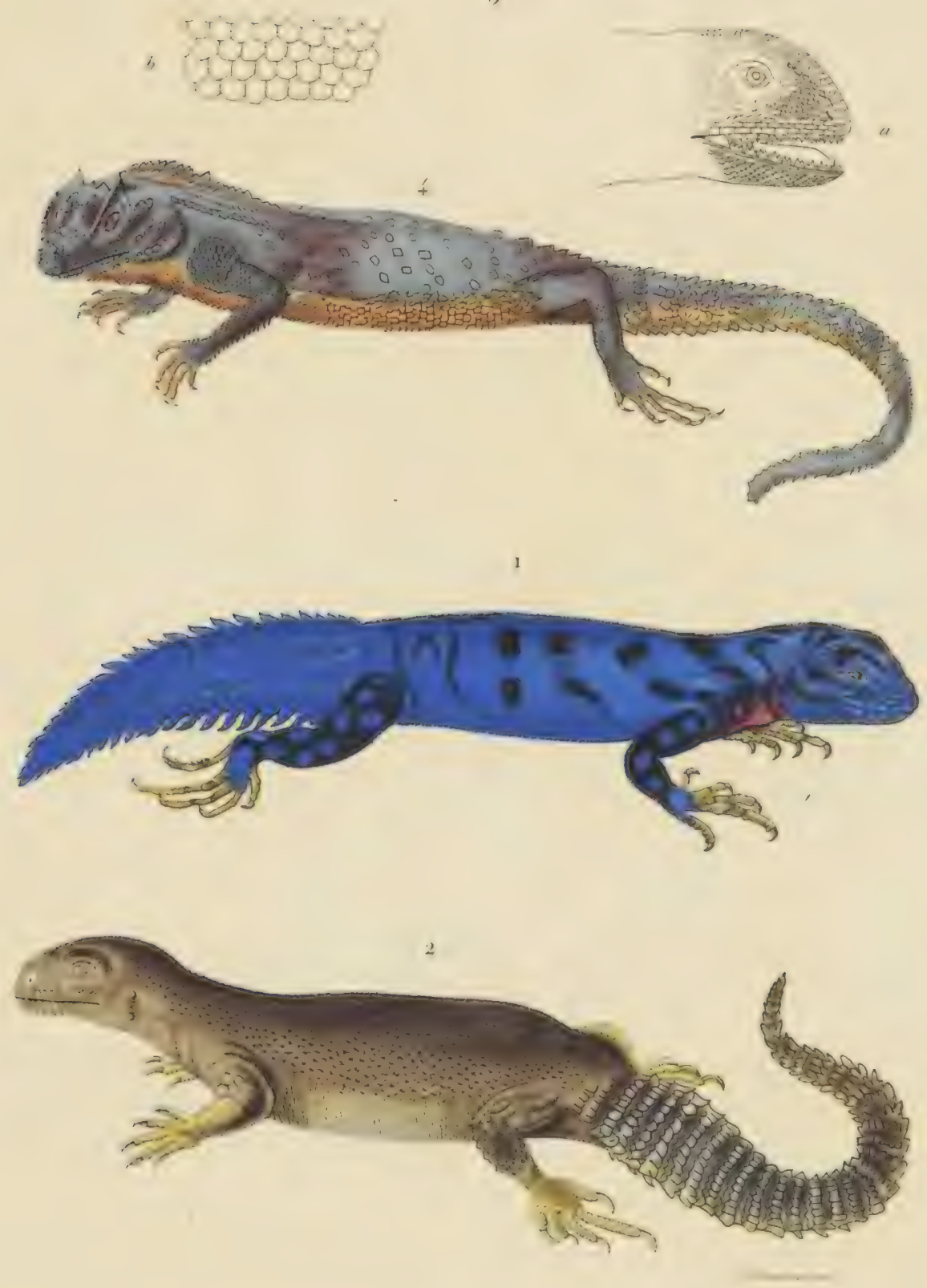

1 DORYPHORE A QUEUE COURTE.

2 FOUETTE QUEUE RÉTTCUAÉ.

3 LETOLFPSIDE TACHETÉ

$f$ LYRTOCEPHALE MARGARITACÉ. 


$$
\frac{1}{E}
$$




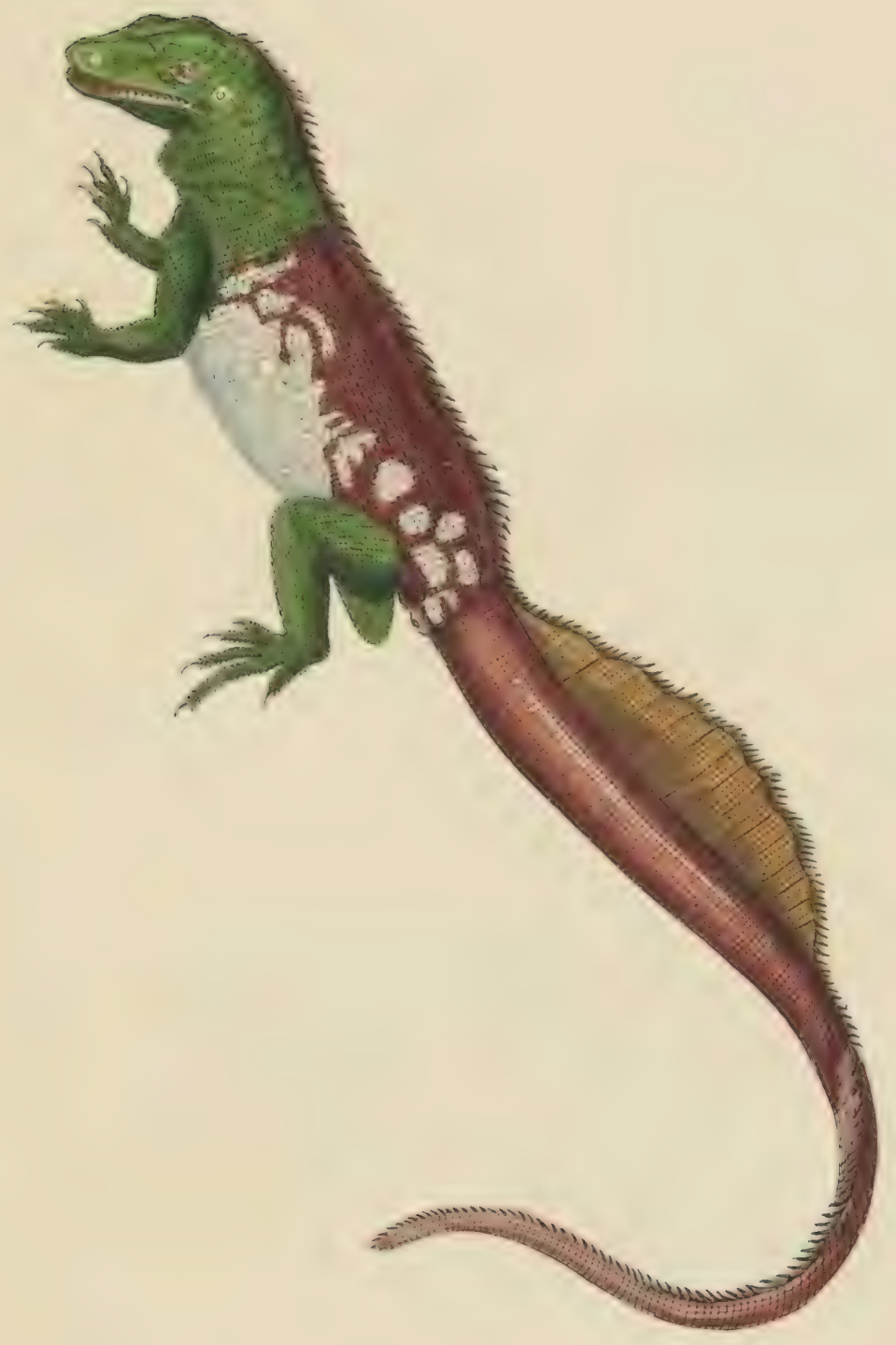

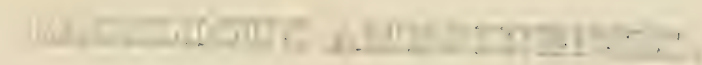

BASIIC D'ATIBOINE. 

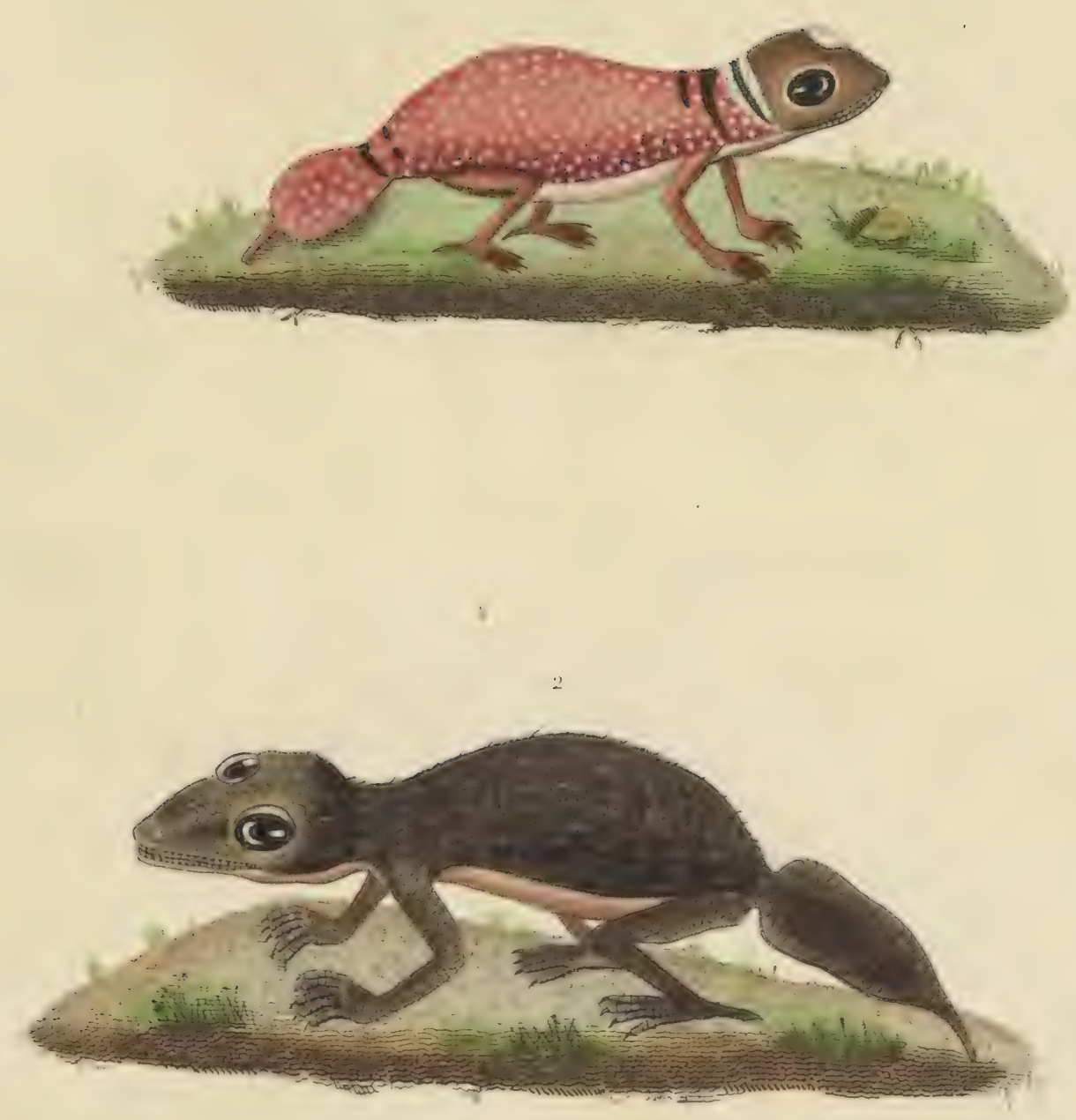

Fs resmares scuip"

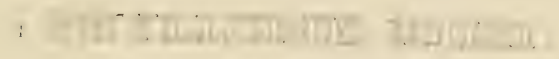

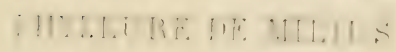

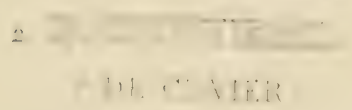




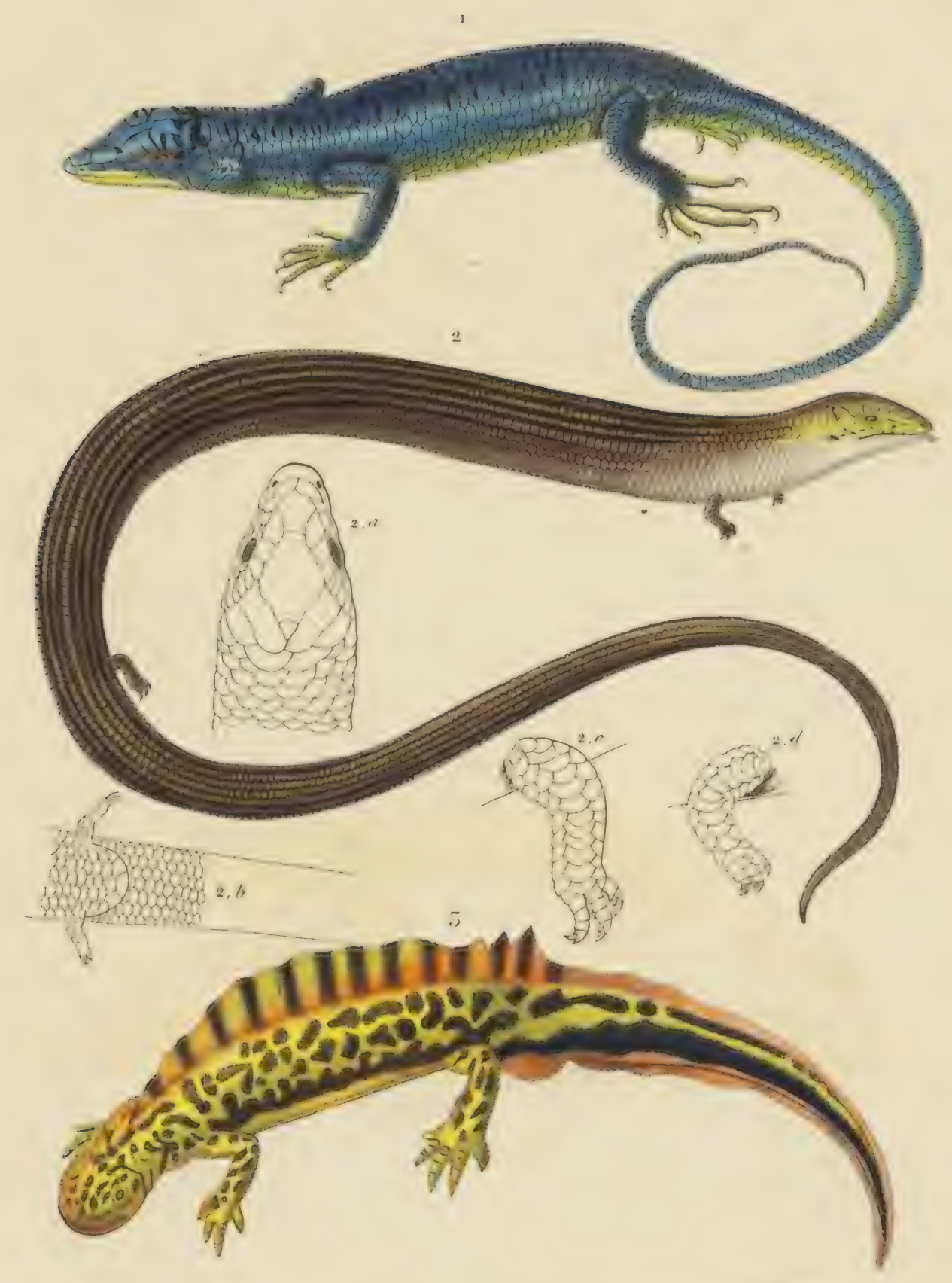

Desmares, se
1 SCINQUE BLEU
2 SEPS STRIÉ.
3 TRITON RAYÉ, 


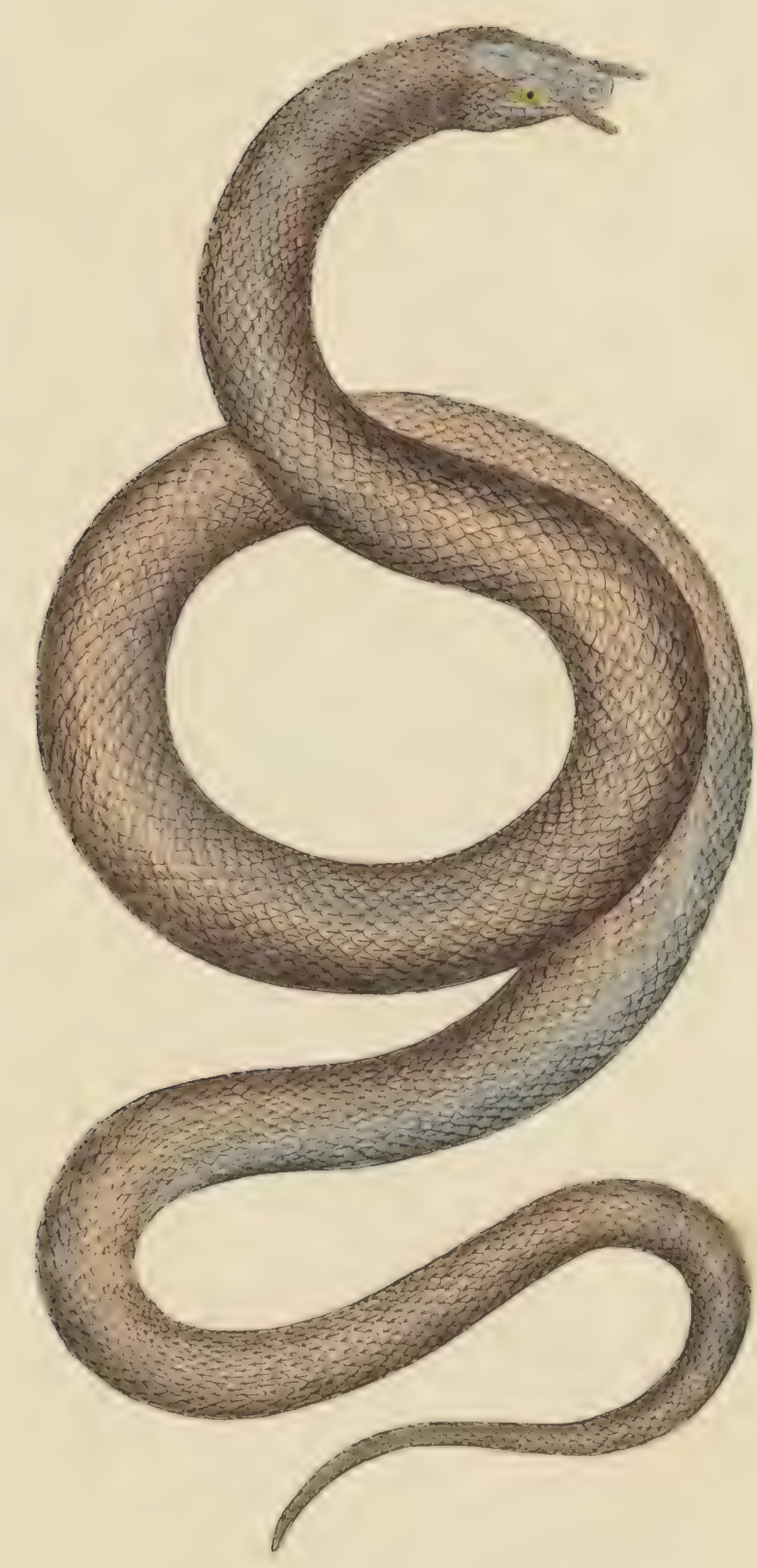

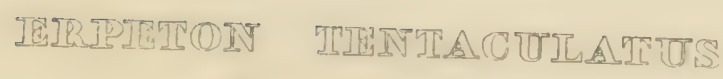
ERPETON TFINTACUTÁ. 

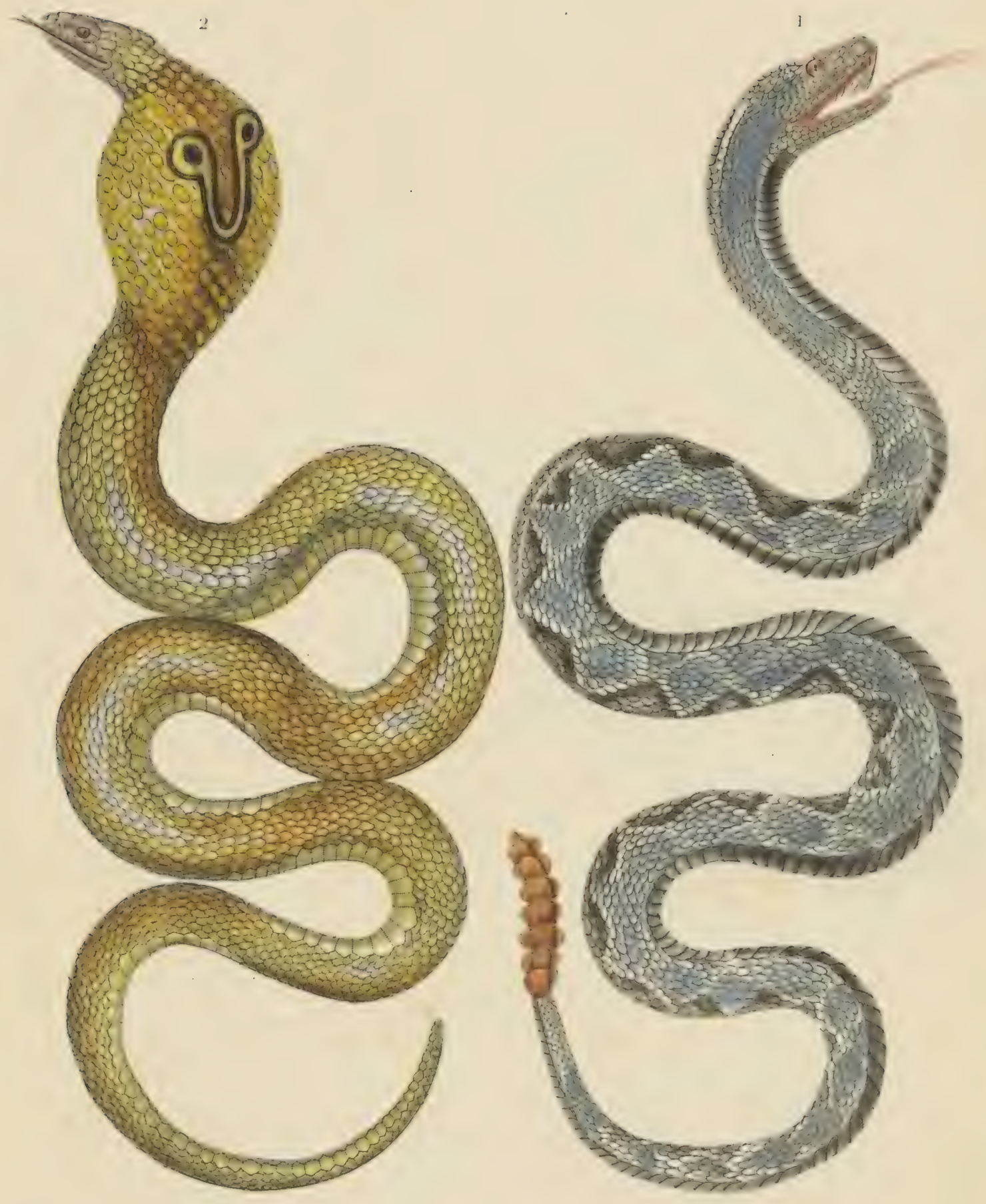

1. CROILLUS RHOMBIFER. Crotale à losanges

¿ NATA LUTESCENS. Naía a lumetles 


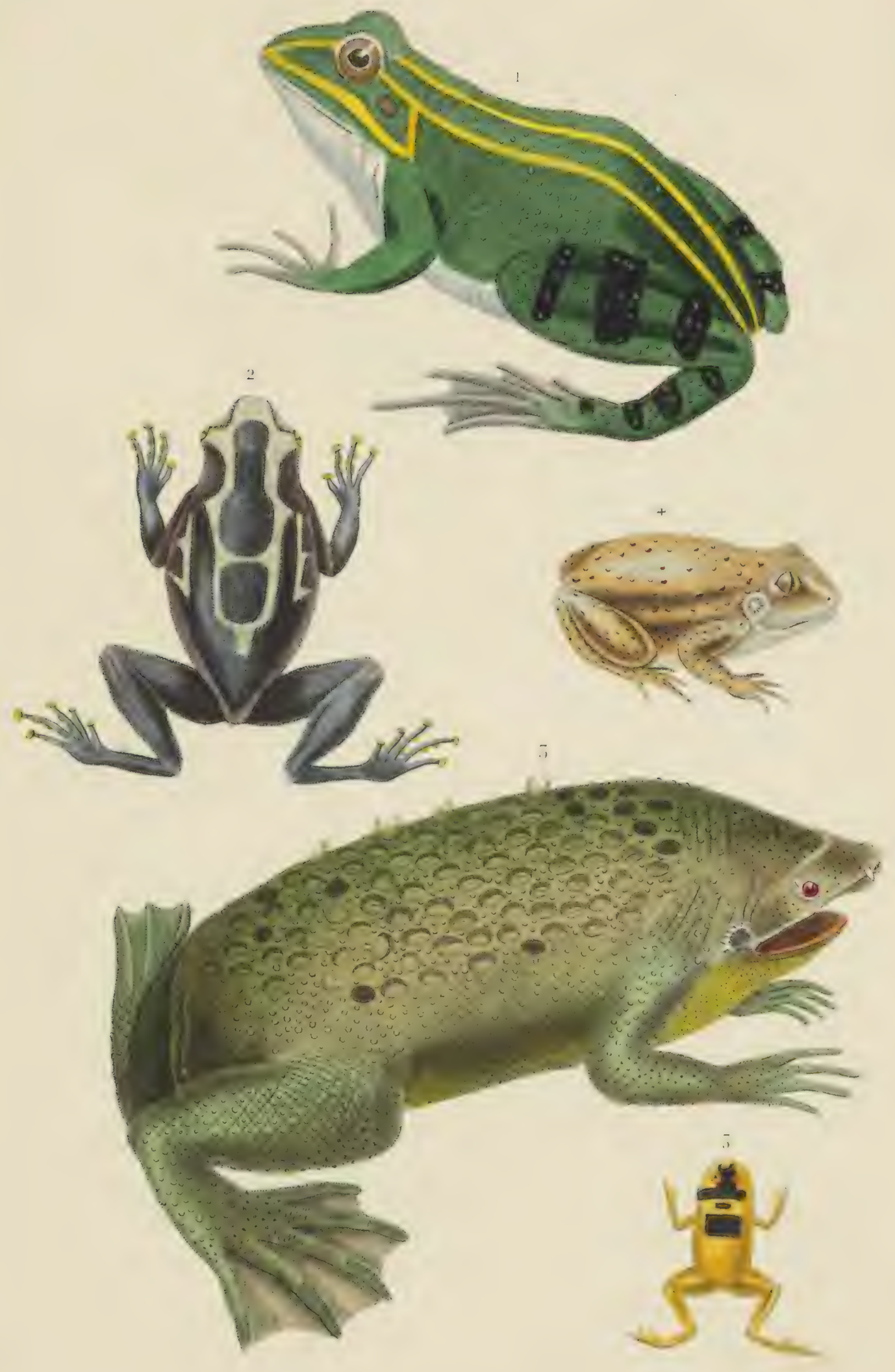

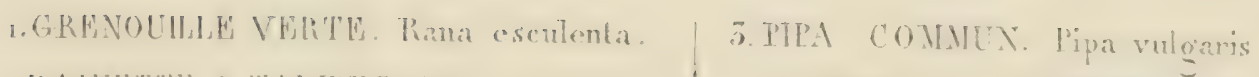

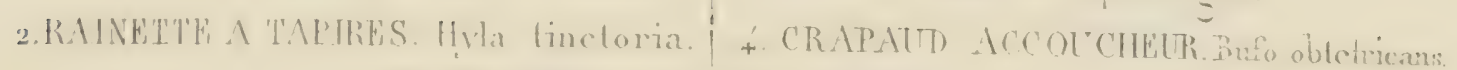

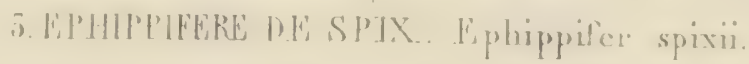




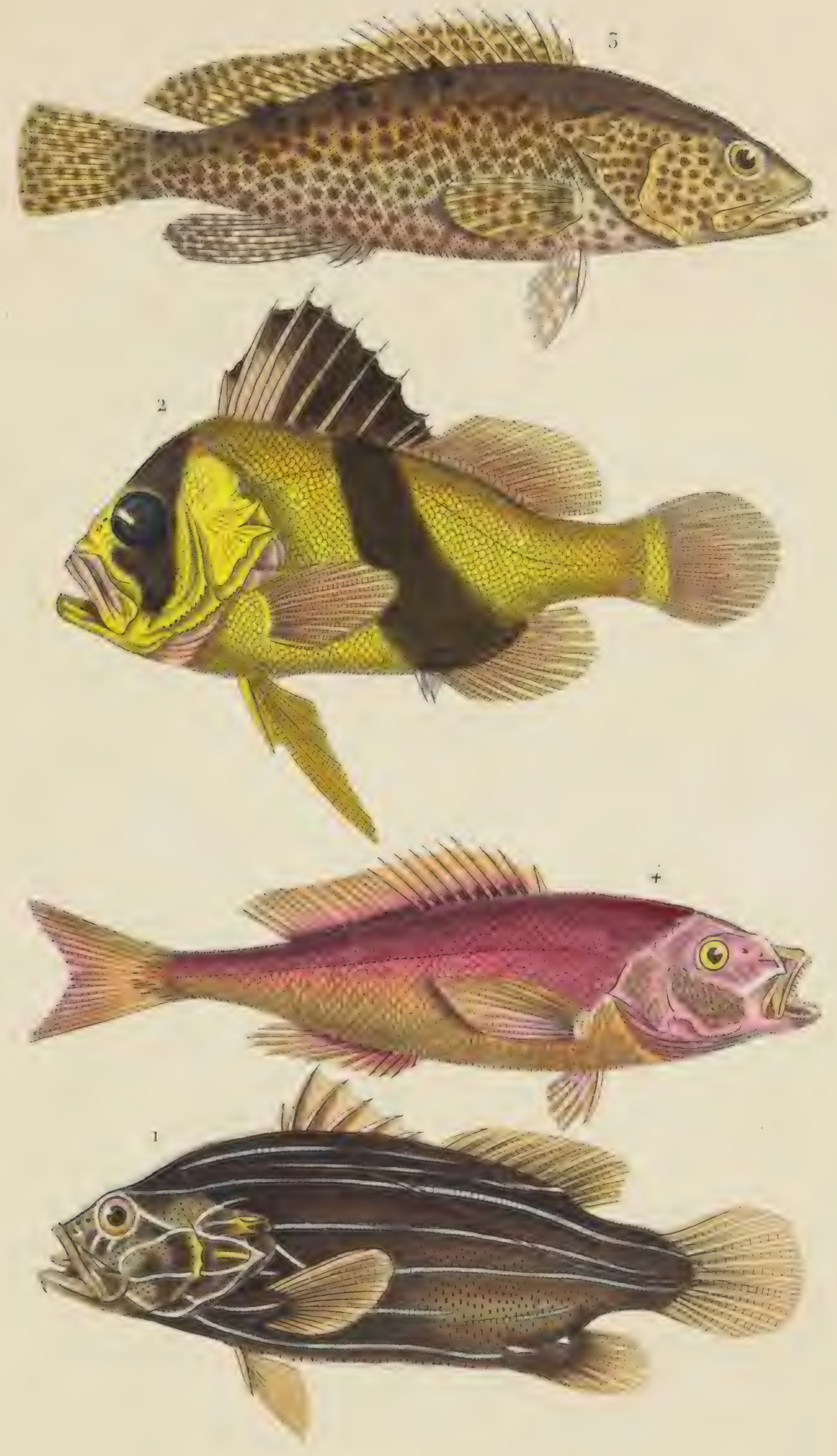

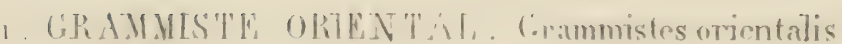

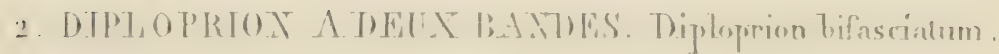

7 SFRRAT IliRol. Sertanus hevagonatus

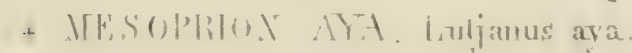




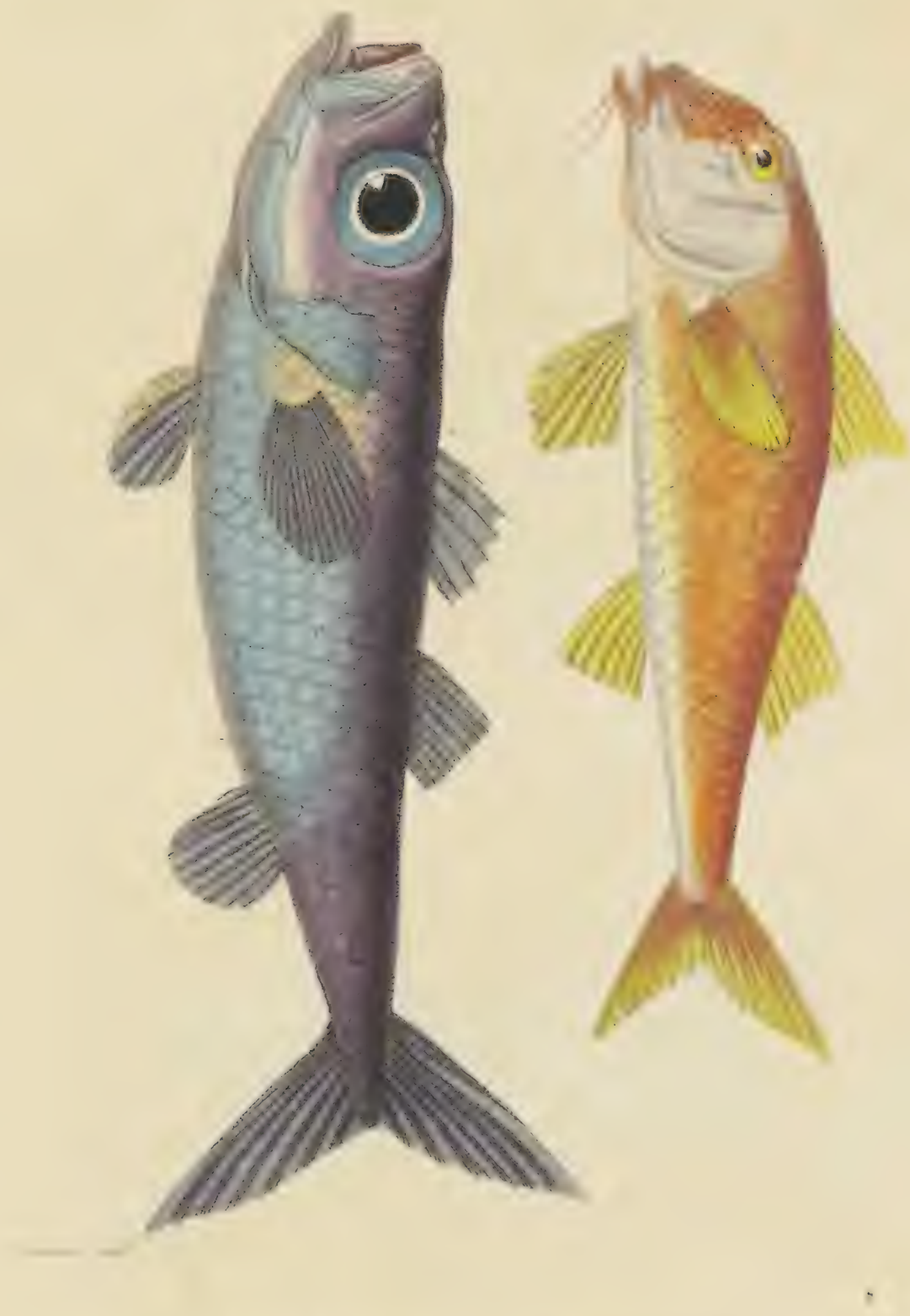

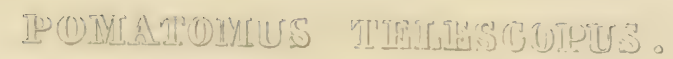

POMATOAE THBSSOPK.

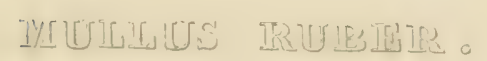

SURMLLAT ROLGET. 


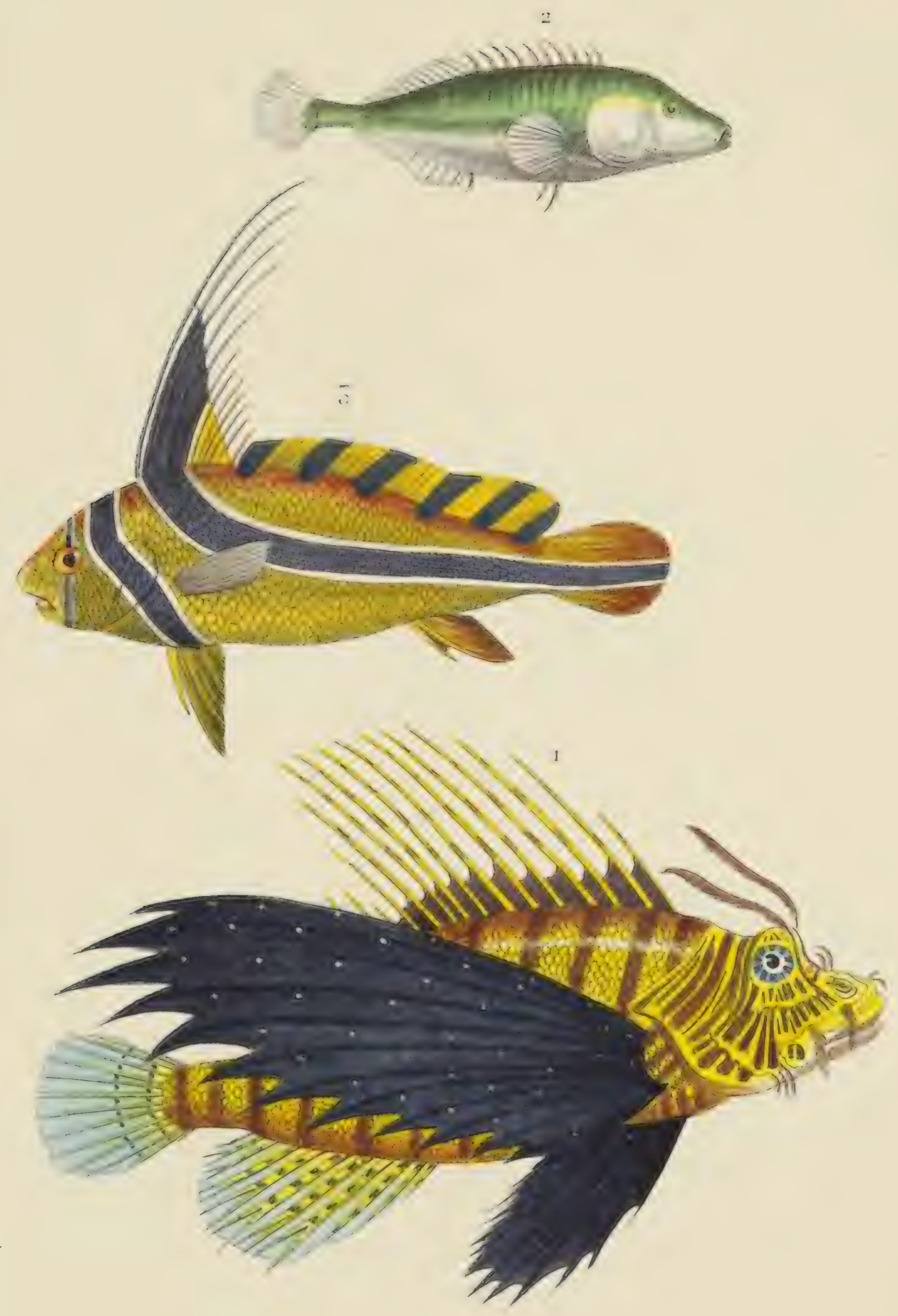

1. PITH DOPEROIS TOLITANS.

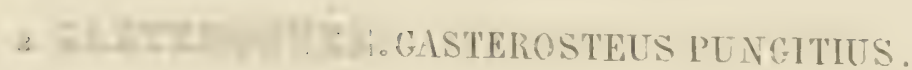

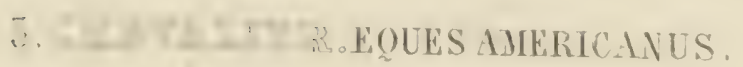


(i).

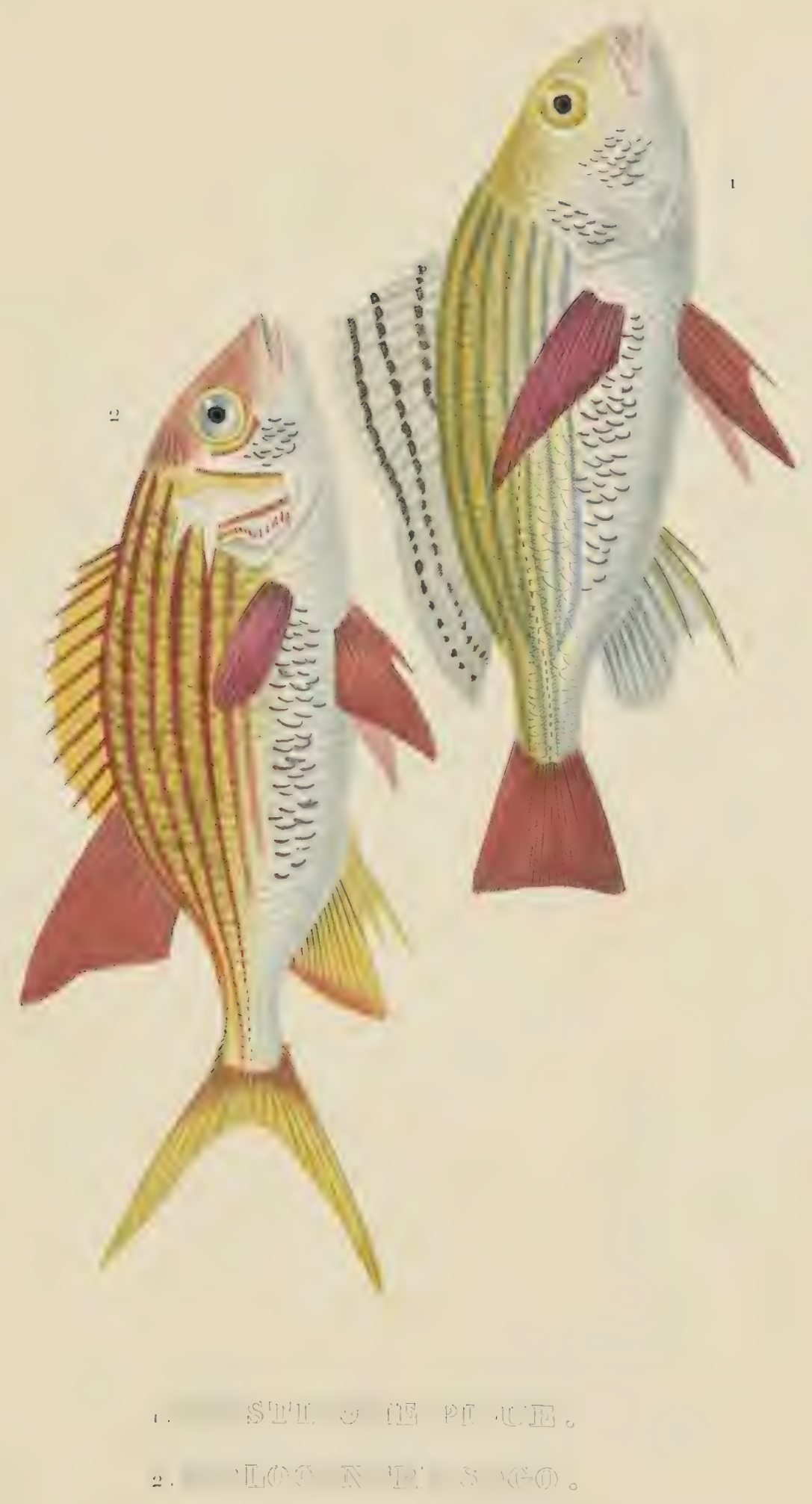




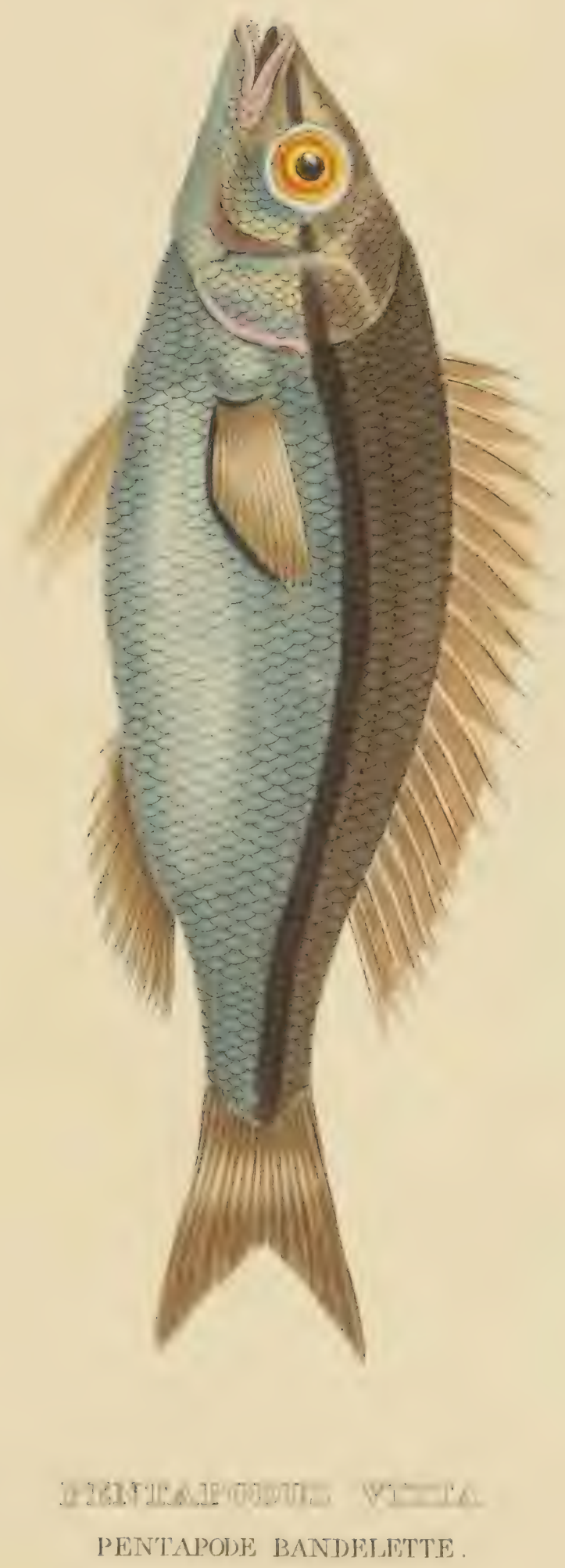




$$
1 !
$$




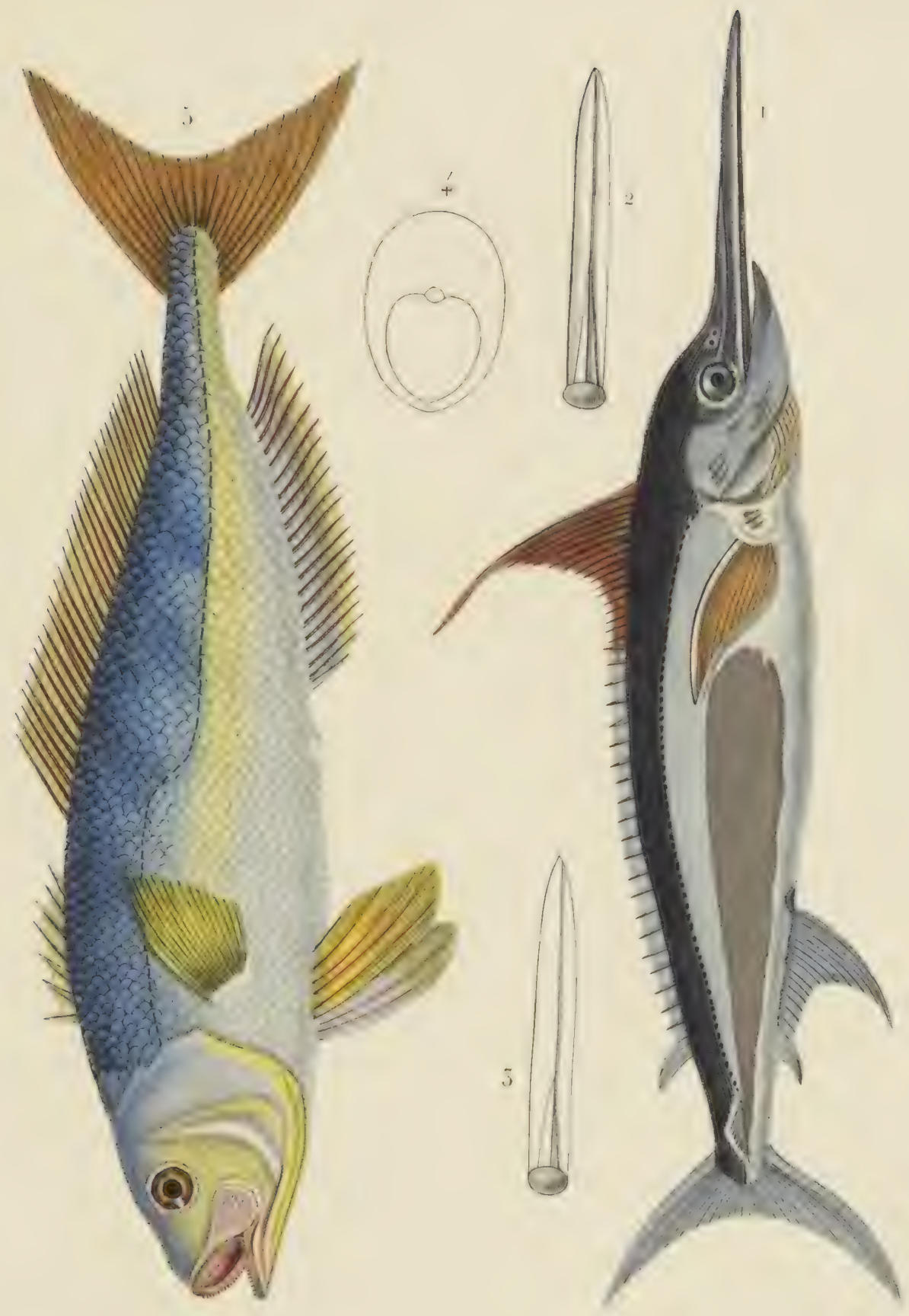

1. ISSPAD ON. Xiphias gladius.

2.3. son glaive. 4.coupe du corps.

5. St'RIOLE. Seriola dumerilii. 


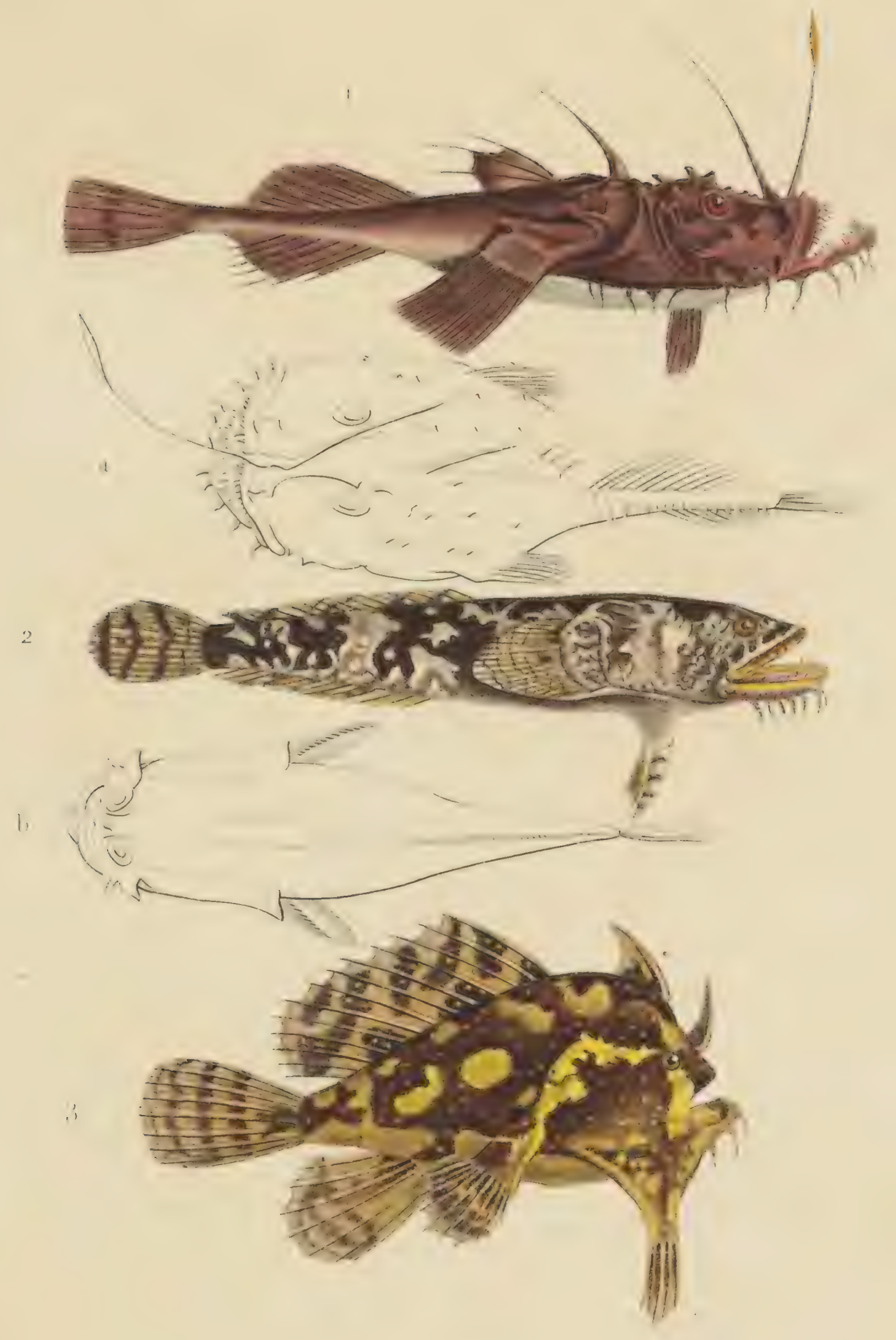

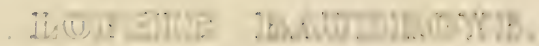

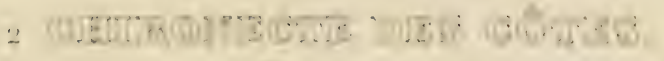

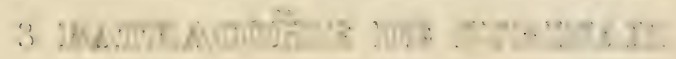




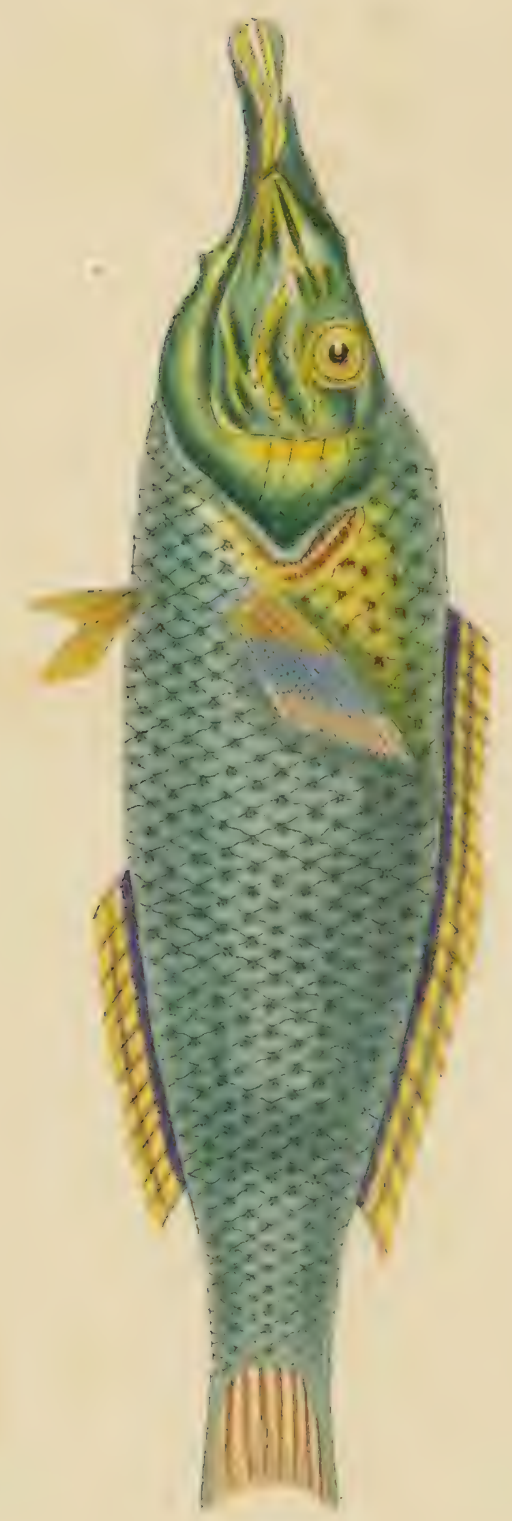

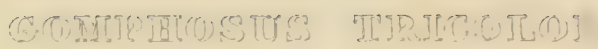
GONIPILSE JACEPEDE. 


$$
11
$$




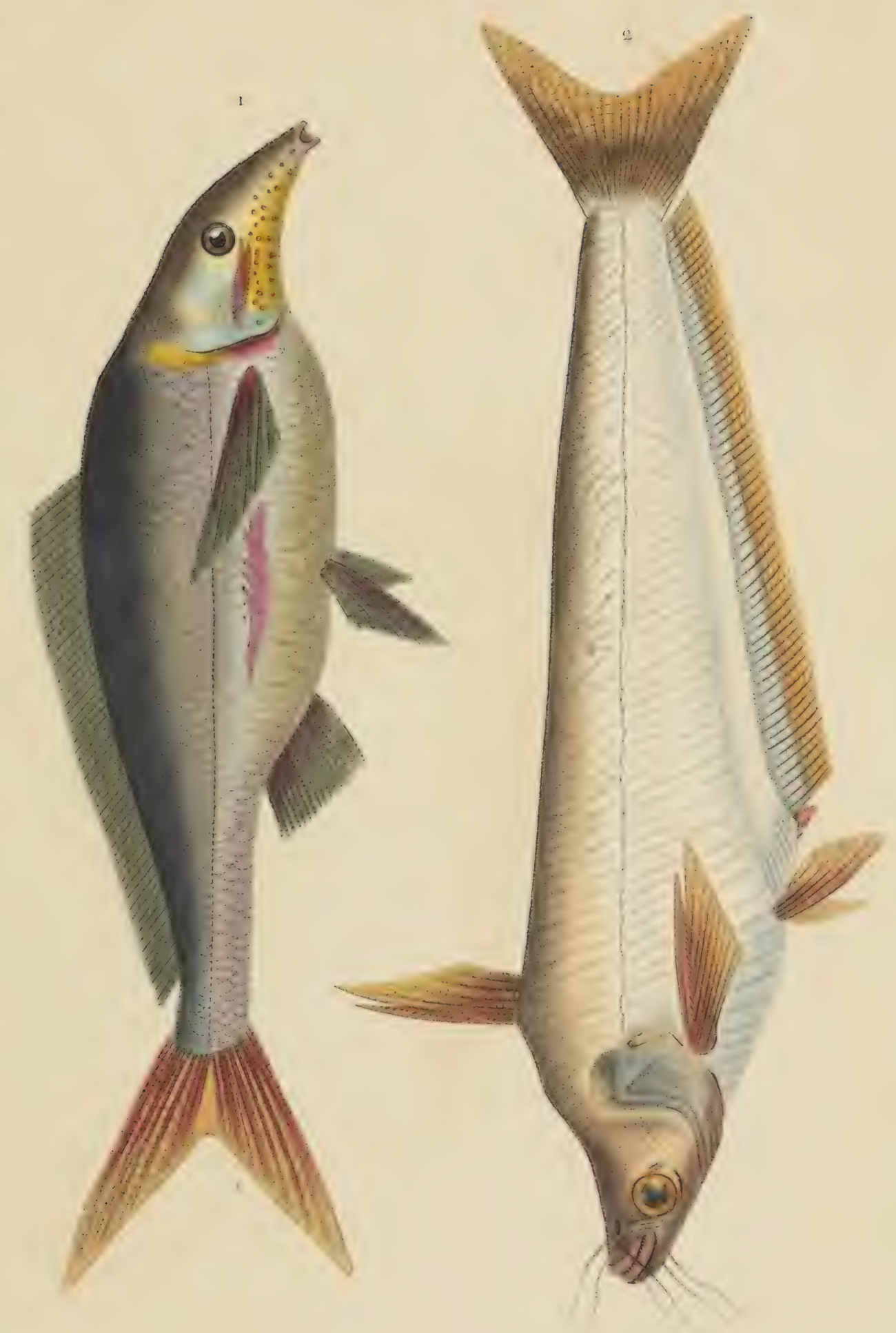

- MIORMYRE OXYRHYMQTE. mormysus oxvernchus

2 SCHTLBF TARBEAT. schilbdus inystus. 


$$
18
$$




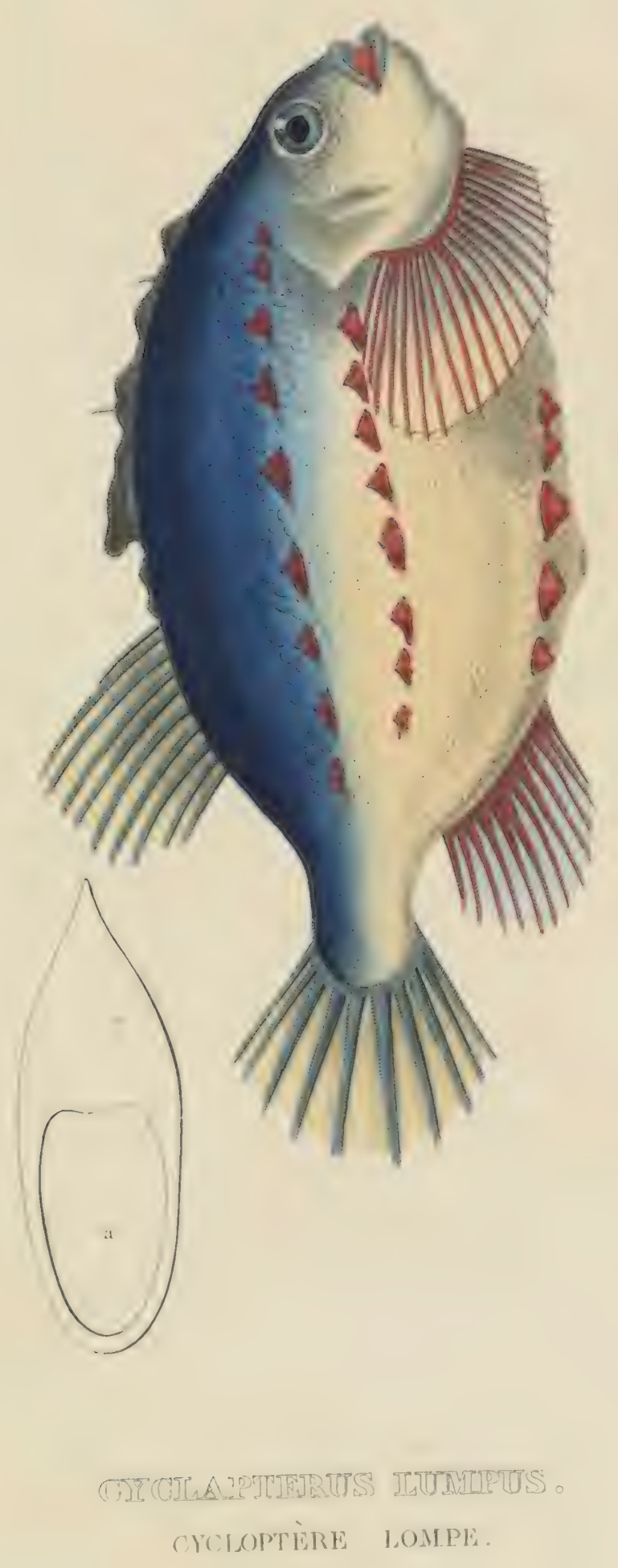




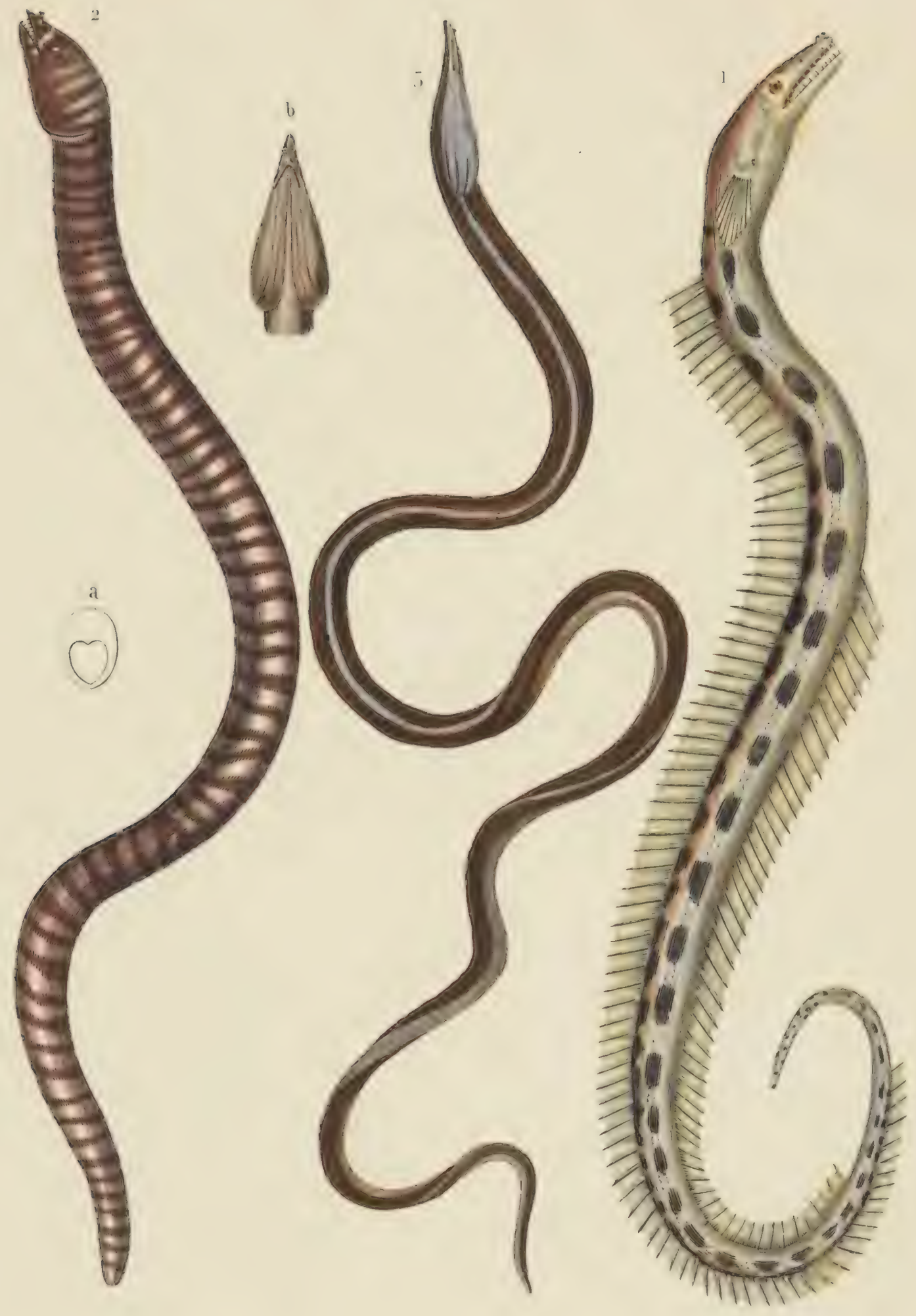

MURFiNE. murena .

1.OPHISURE serpent de mer.

2.MURENE zébre a.coupe du corps.

3. SPHAGËBRANCHF aveuole. b. tète rue en dessous. 

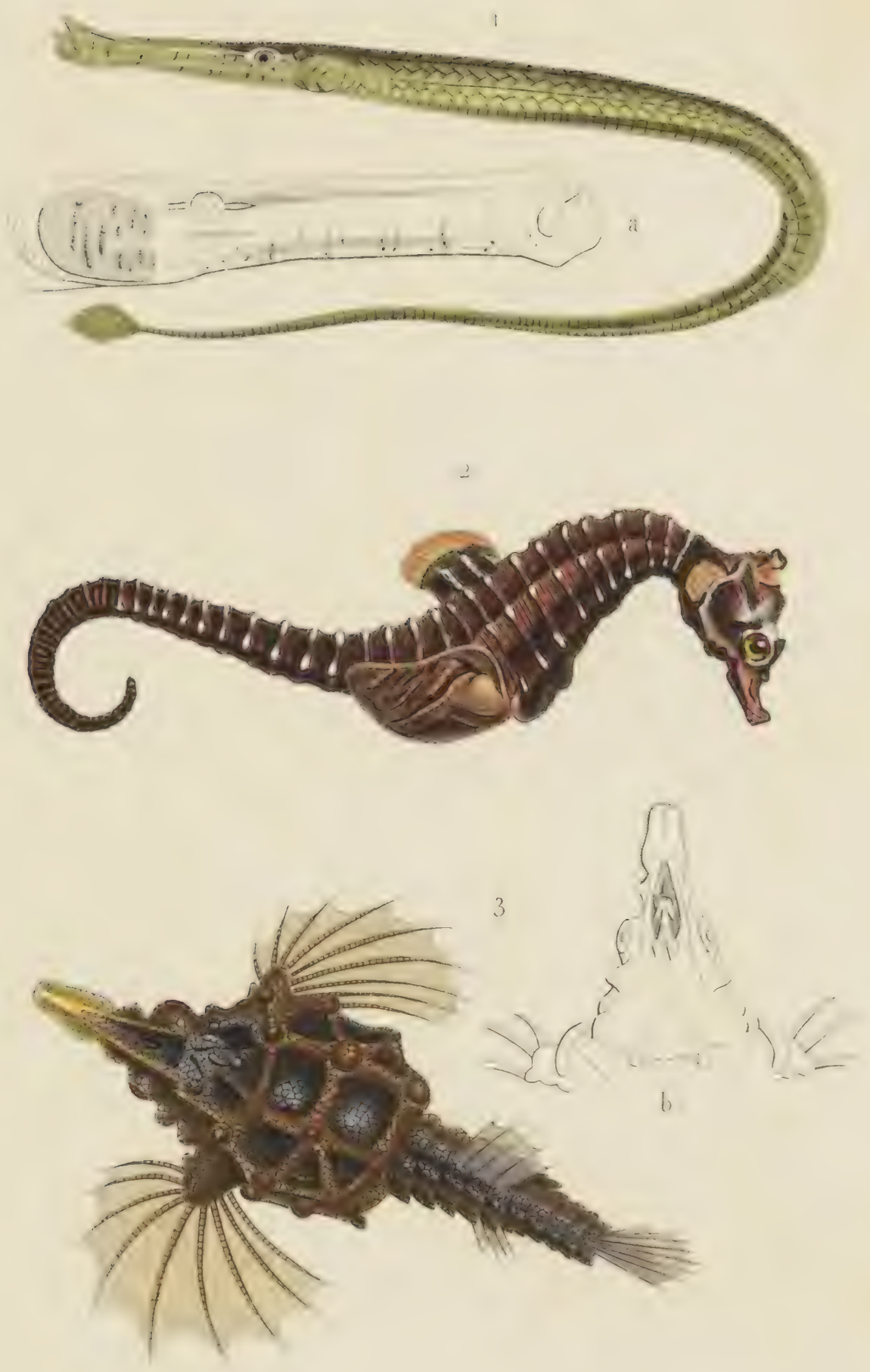

1. EMH

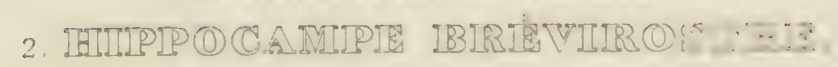

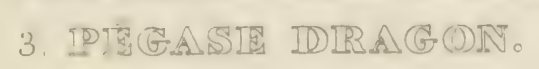



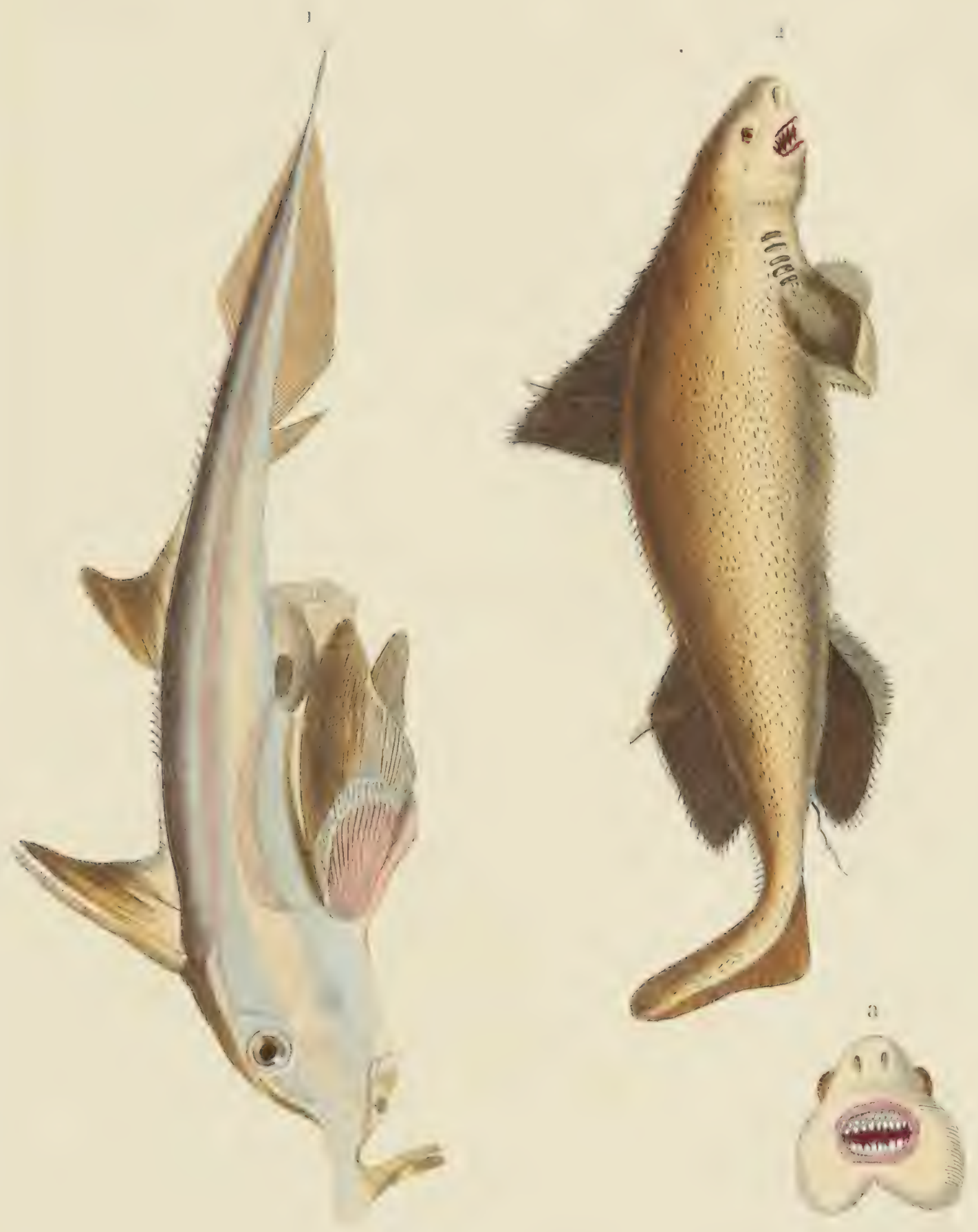

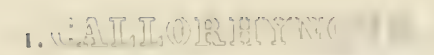

CALIORHYACHUS ANTARTICUS.

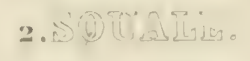

SOUNLS CRNTRINA. a.La têtevue en dessous. 


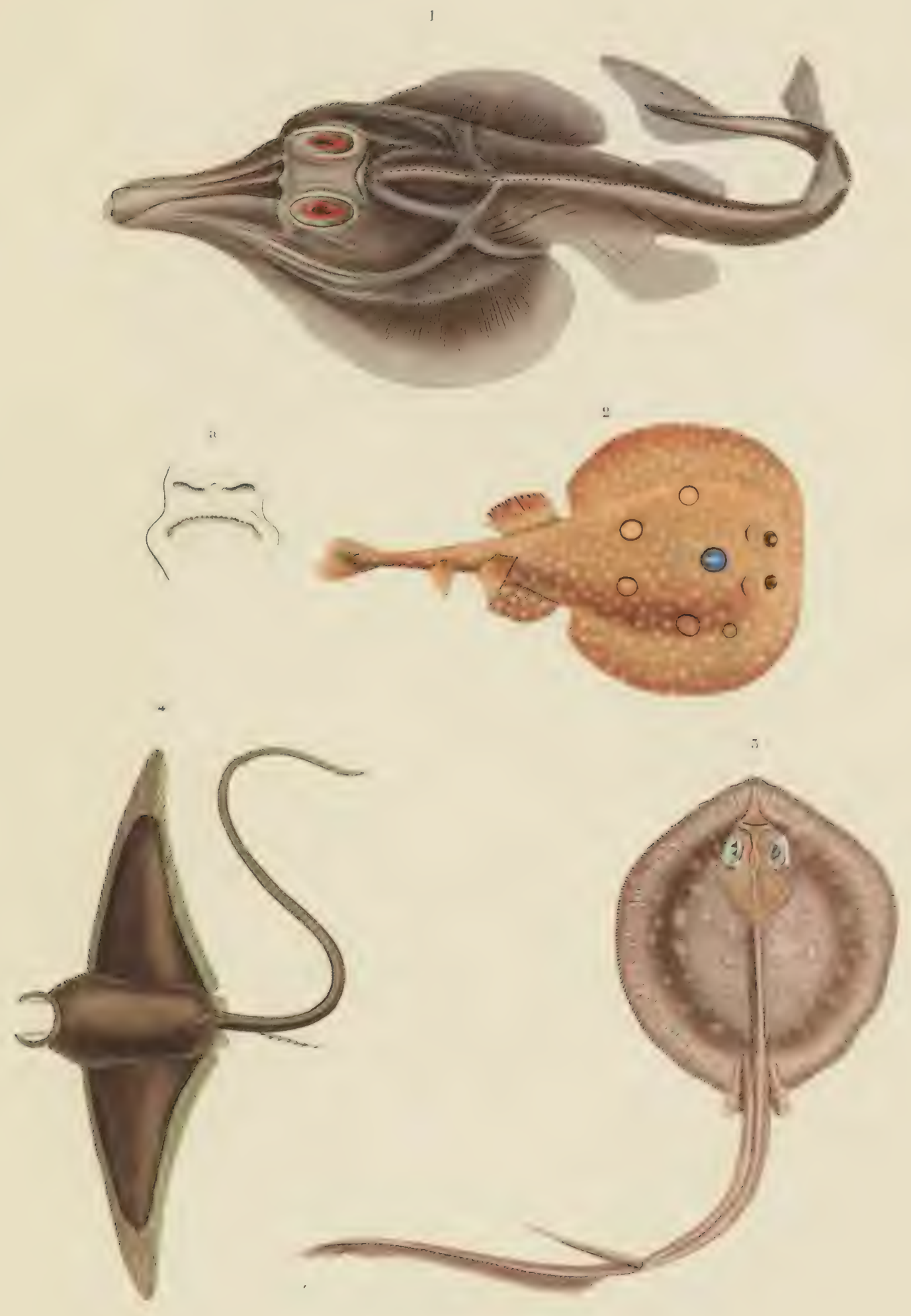

1. RHNOBATE LISSE a ses madinites

2. IORPILLE NARKE.

3. TRYGON DE HALGAN.

4. CÉPHALOP'TERT: GIORNA. 

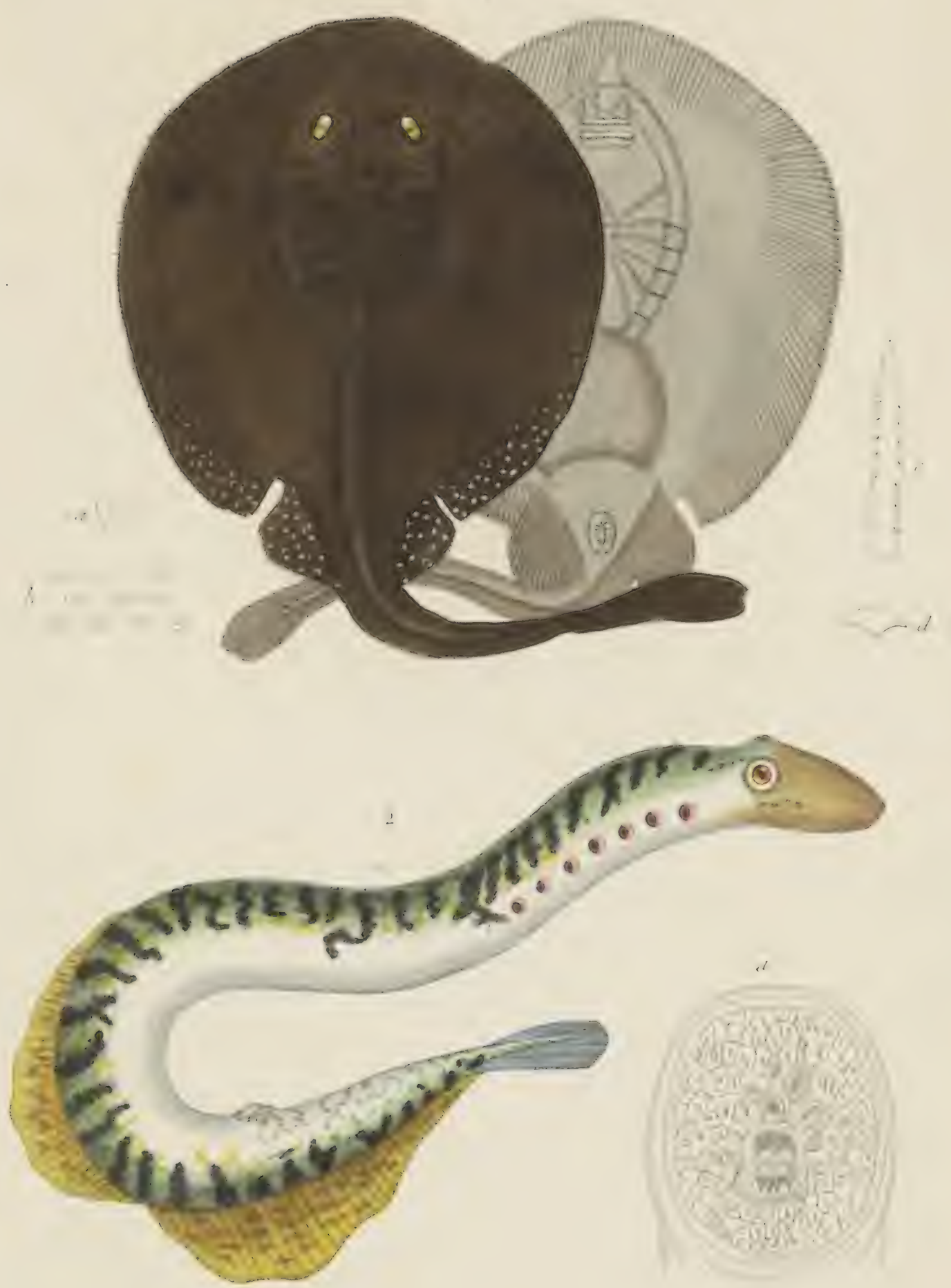

- TRYGON TORPEDTNES Raie-pastonague torpedine.

2. PETRUMHZON MARIXUS Petromizon Lamproie. 


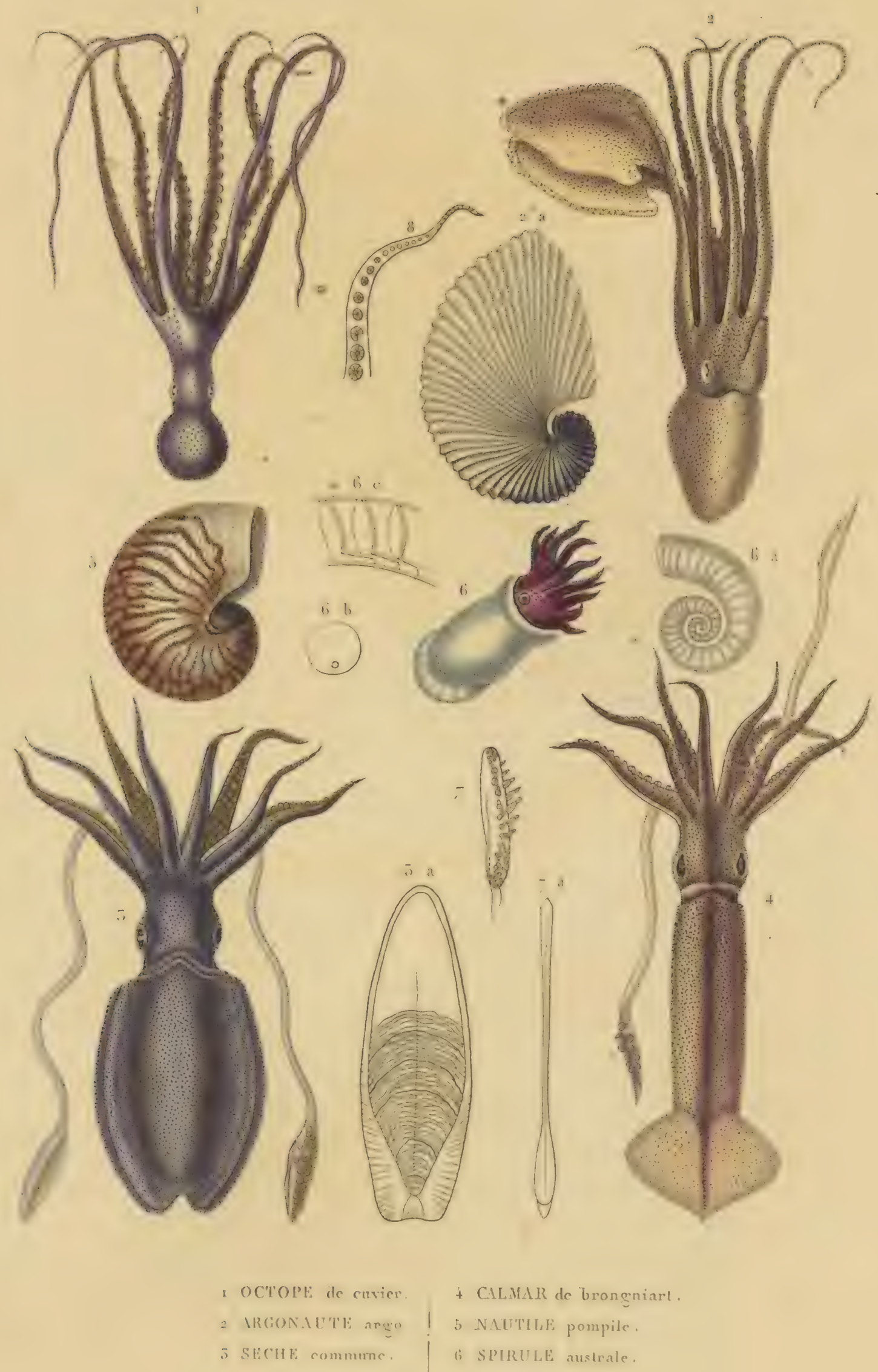

l'ragment d'un bras foNTCIOTEUTIIS. 


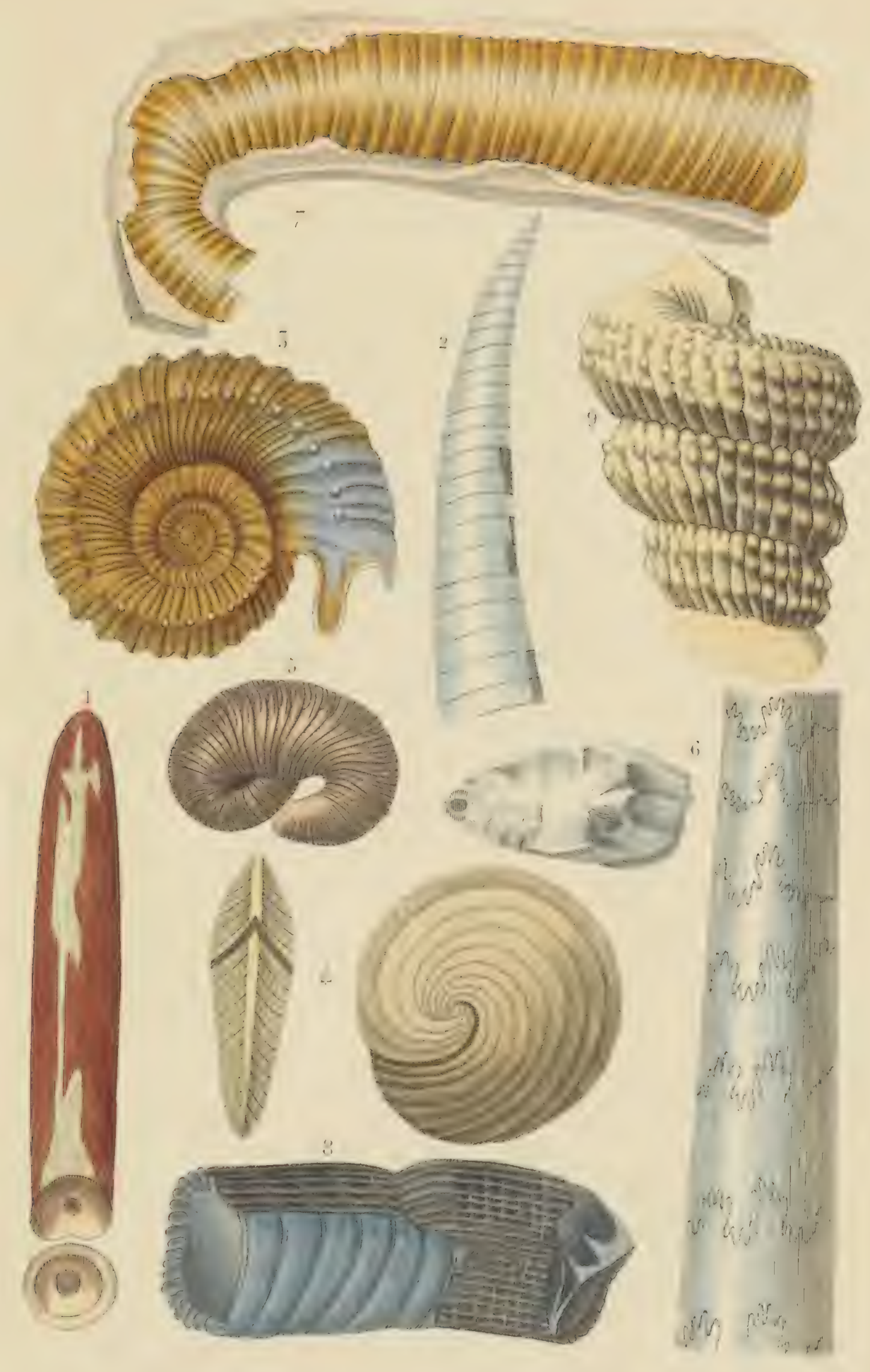

1. BFiLEMNITE d'osterfield. 5. SCAPHITE oblique.

2. CONHITE unguliforme.

6. BACUI,ITE rertébrale.

3. AMILONITF de Bayeux.

7. HAIITE cylindrique.

1. ORB [CUITE numismale.

8. AIPLEXIS coralloïde.

9. TURRILTTE turbinée; var. 

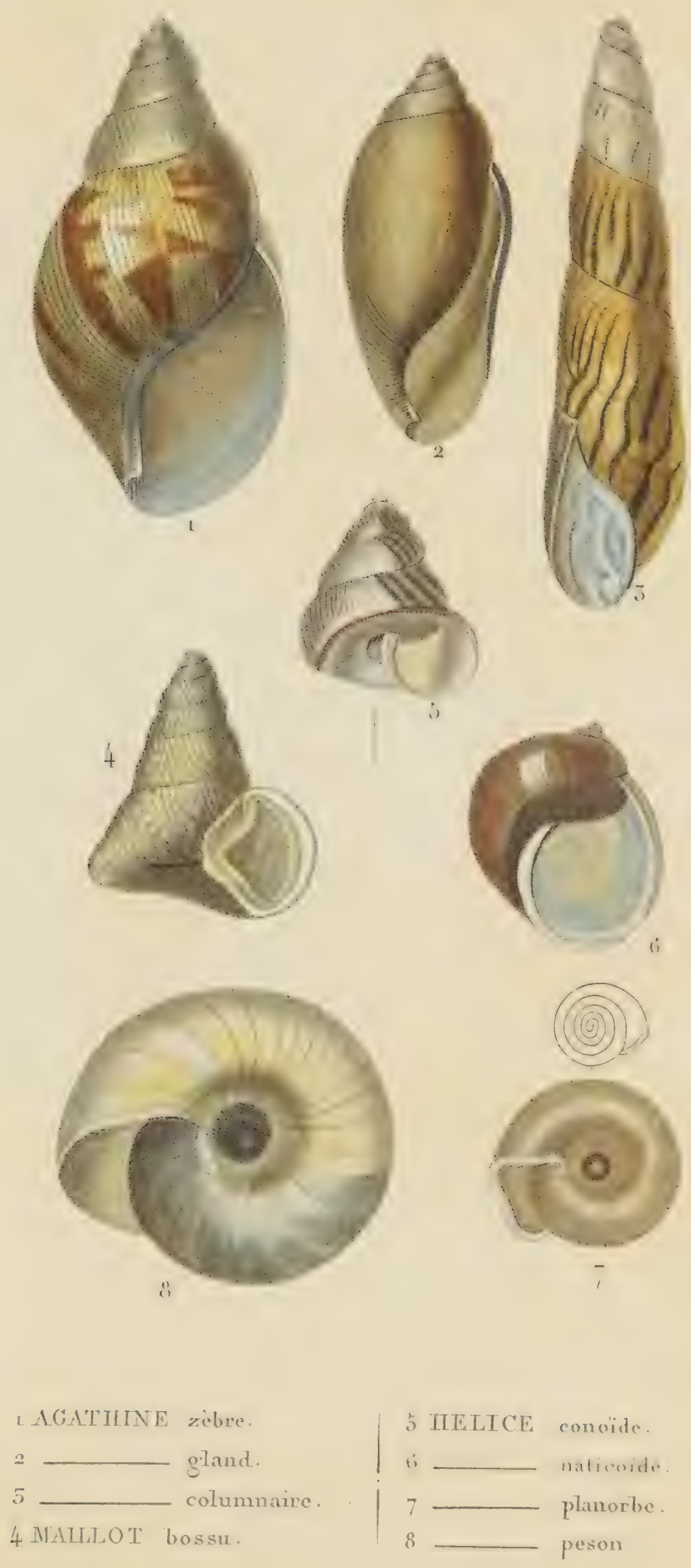


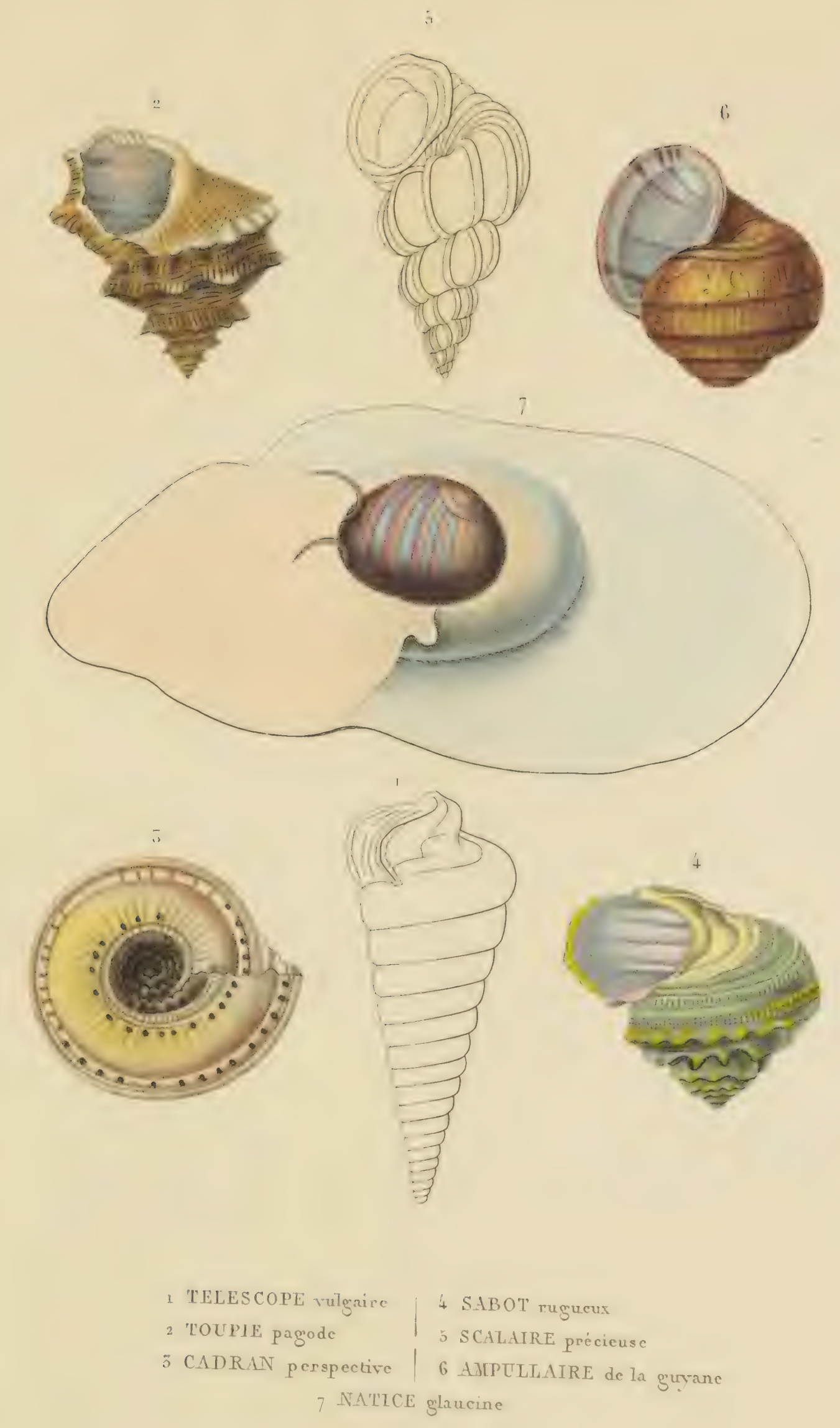

nermares ife 

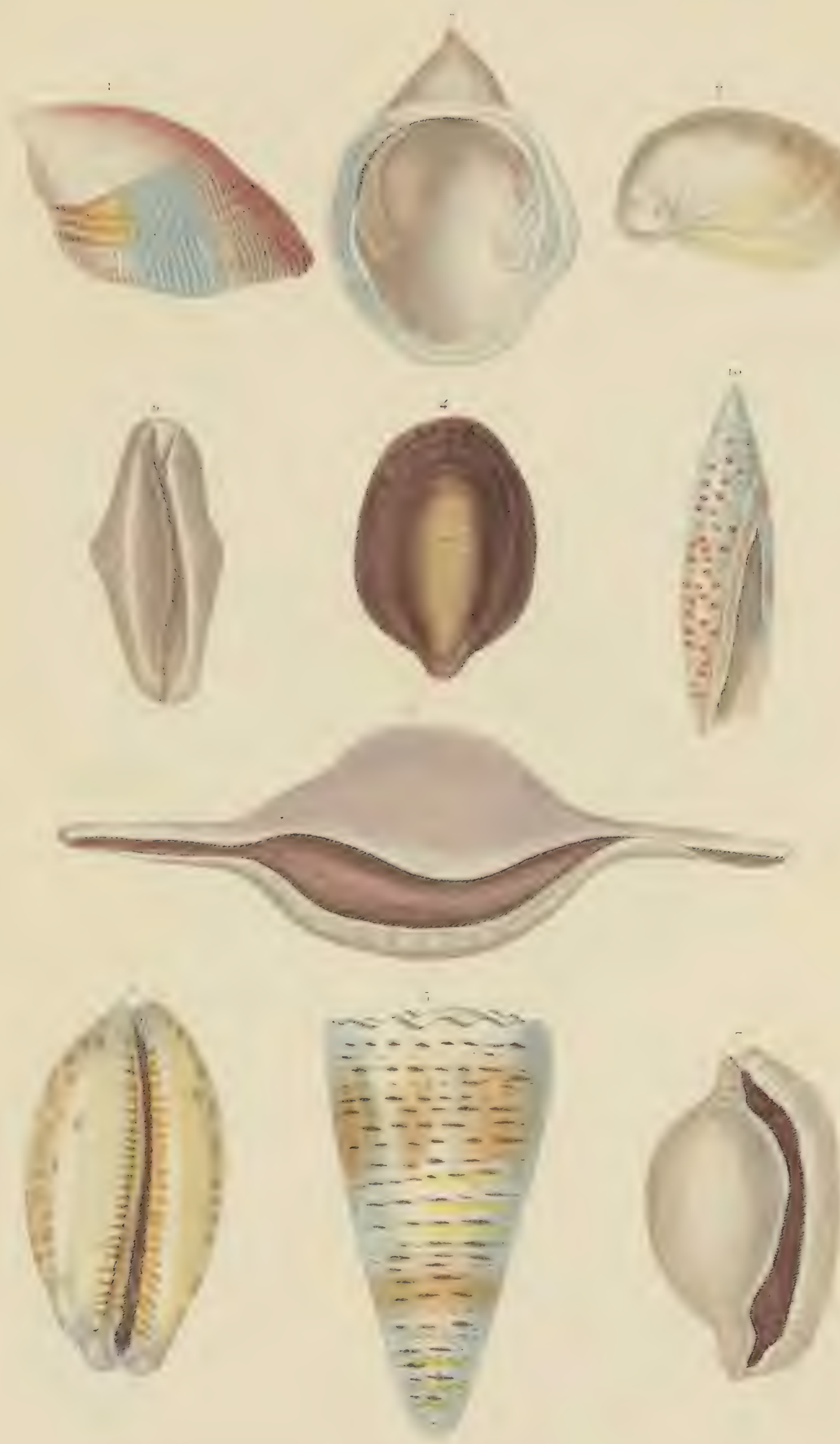

1.2.11PPOXIC': Corne d'abondanee.

3. (RREPIDLTE subspiré.

4. X.HTCTELLF elliptique.

3. Cônti imperial.

6. PORCFTALI colantine. 7. MULF des moluques. j 8. N.ITFThF, volve. a. CliTLAE vibleux

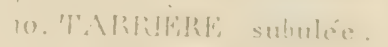




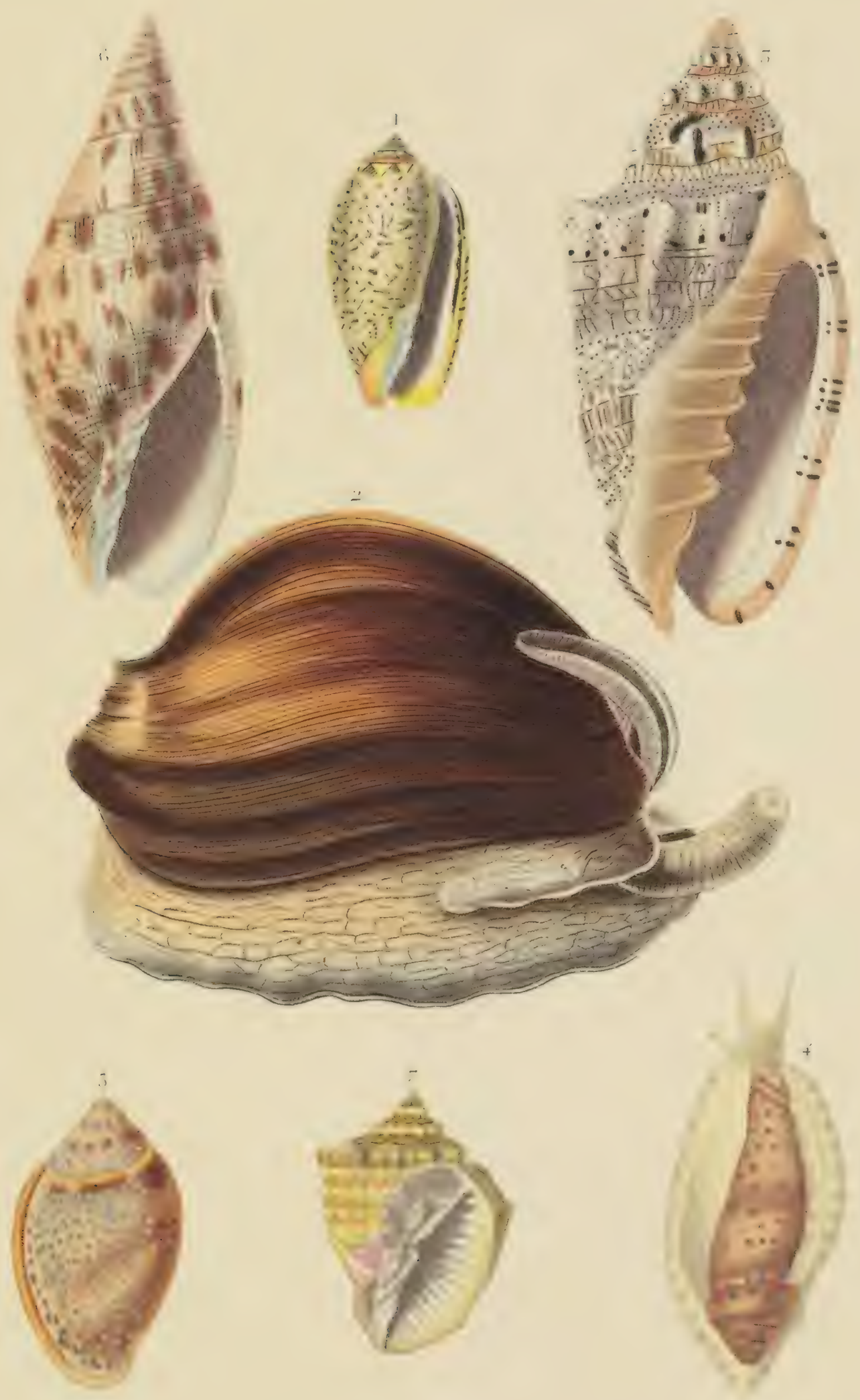

7. OlIVE polpasta. 4.5.MARGLELTE neigeuse.

2. VOLUTE de neptune. $;$ 6. MITRE de ramarek.

5. VOLUT'F, thiarelle. T. CANCELLAIRE impériale. 


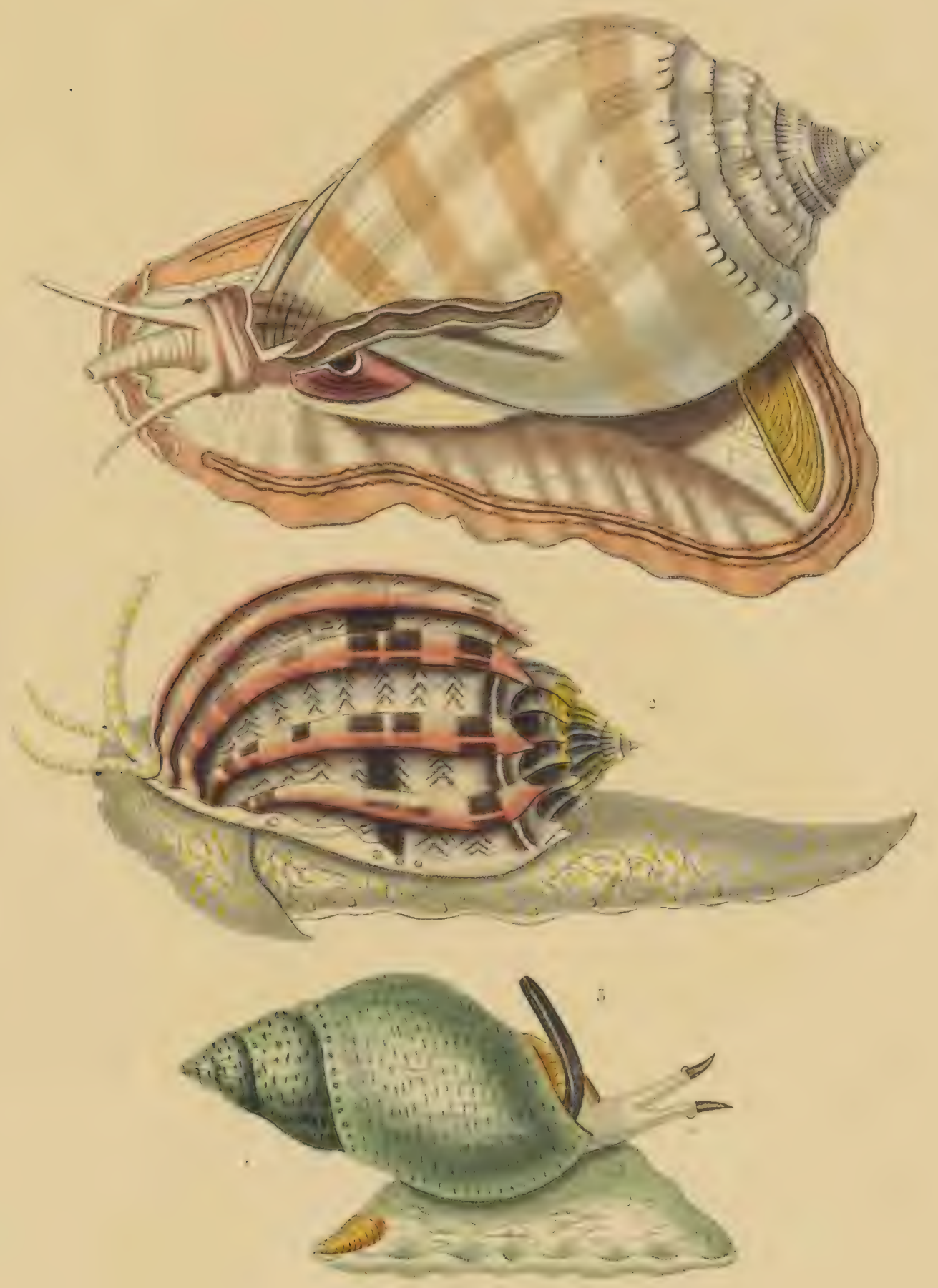

CASQUE BEYOAR. Cassis plancr.

2 IIARPE VEN'TRUE. Ilarpa ventricosa

; BUCCIN ÉCAILLE. Buccinum testudineum 


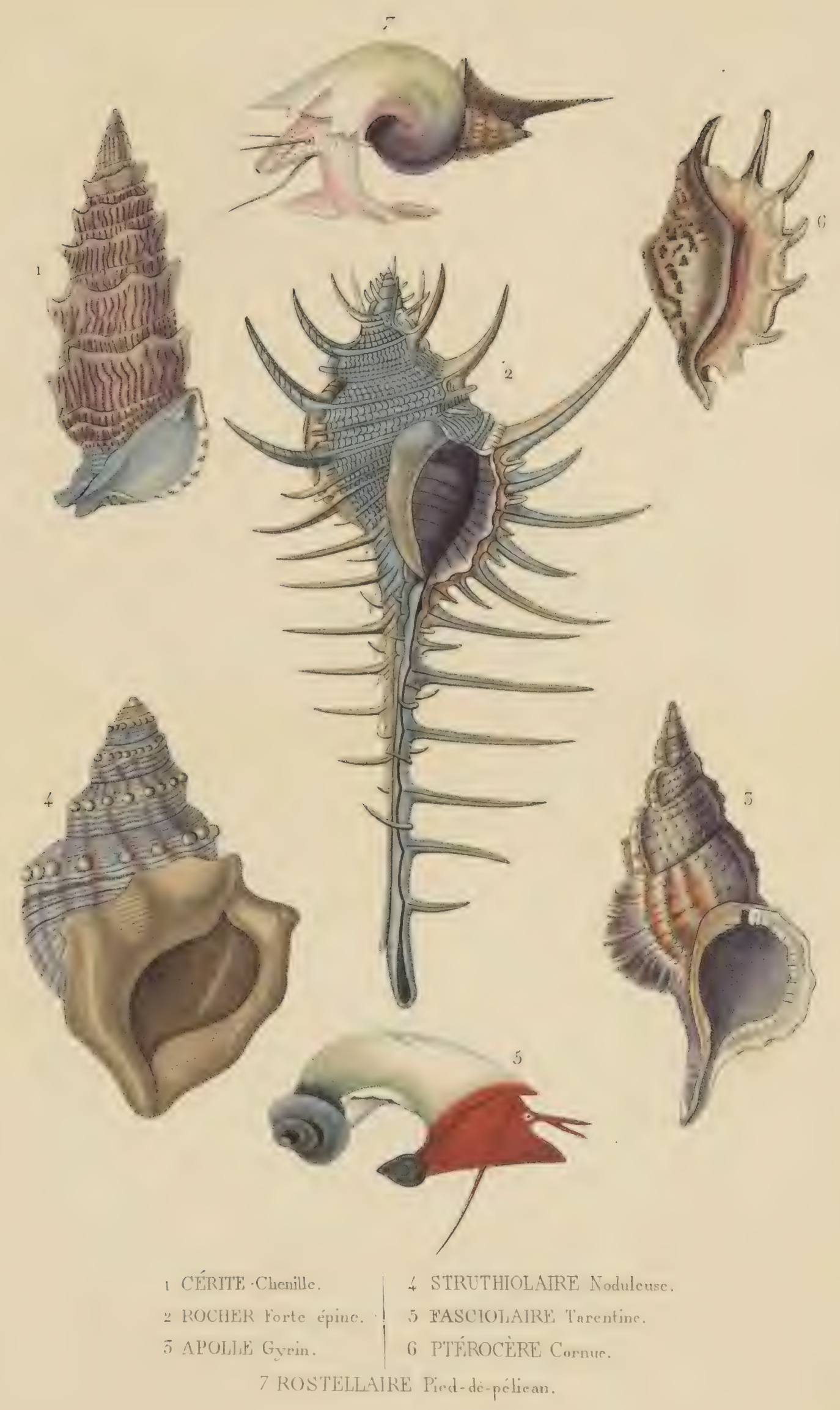




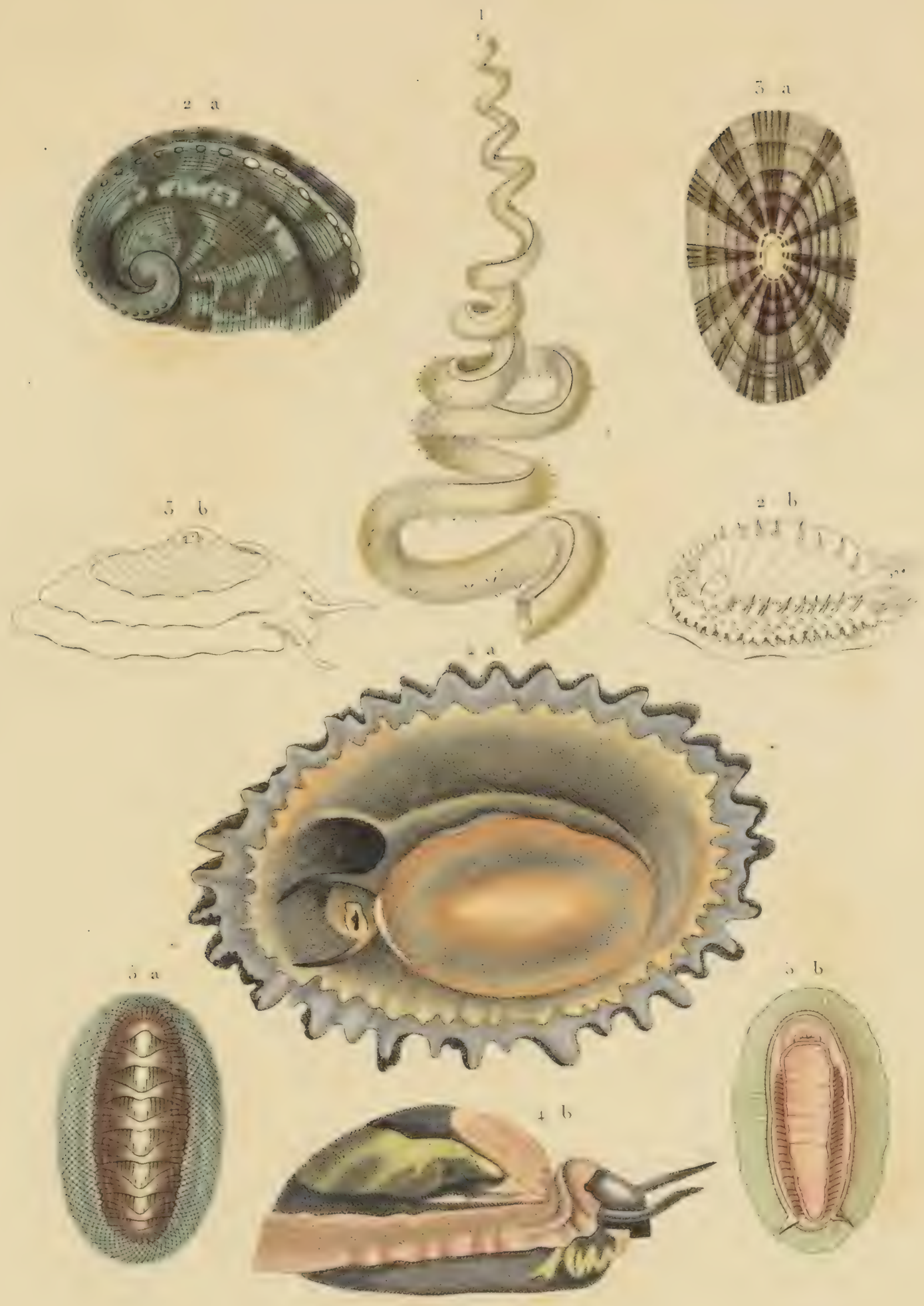

I SIIACALE muriquee 2 a et 6 HALIOCJDE canaliculé 14 a et $b$ PATLLLE pramidé 5 OSCABRION érailleux 


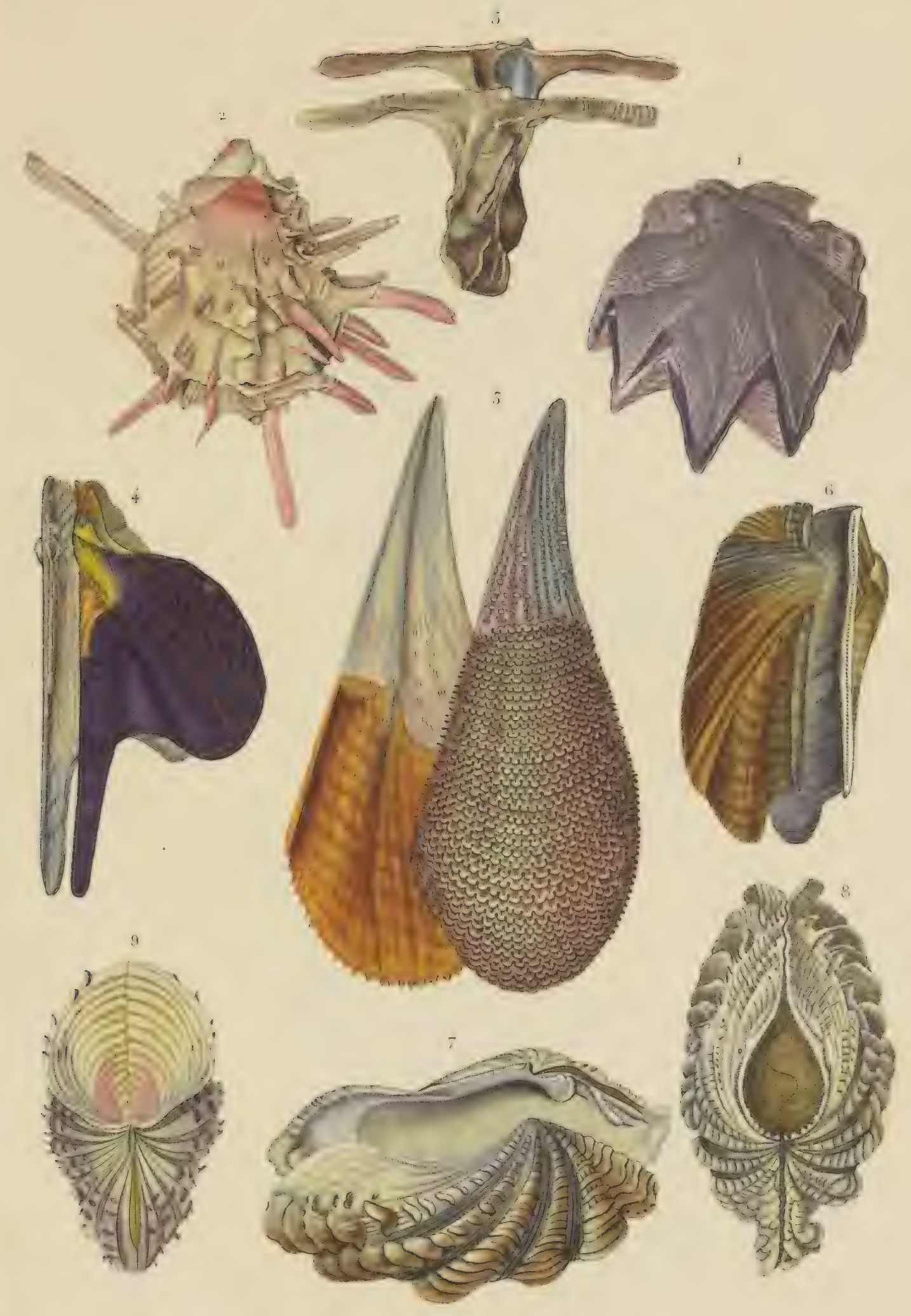

1.HITIRH erçle de coq. 5.PINNli hérissée

2.SPONDYJW américain | G.PHTONCLE pectiniforme.

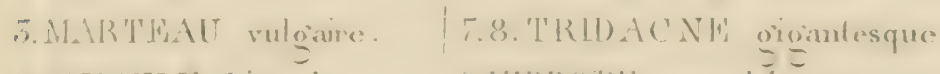

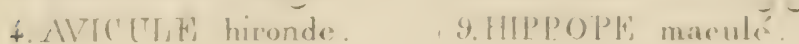



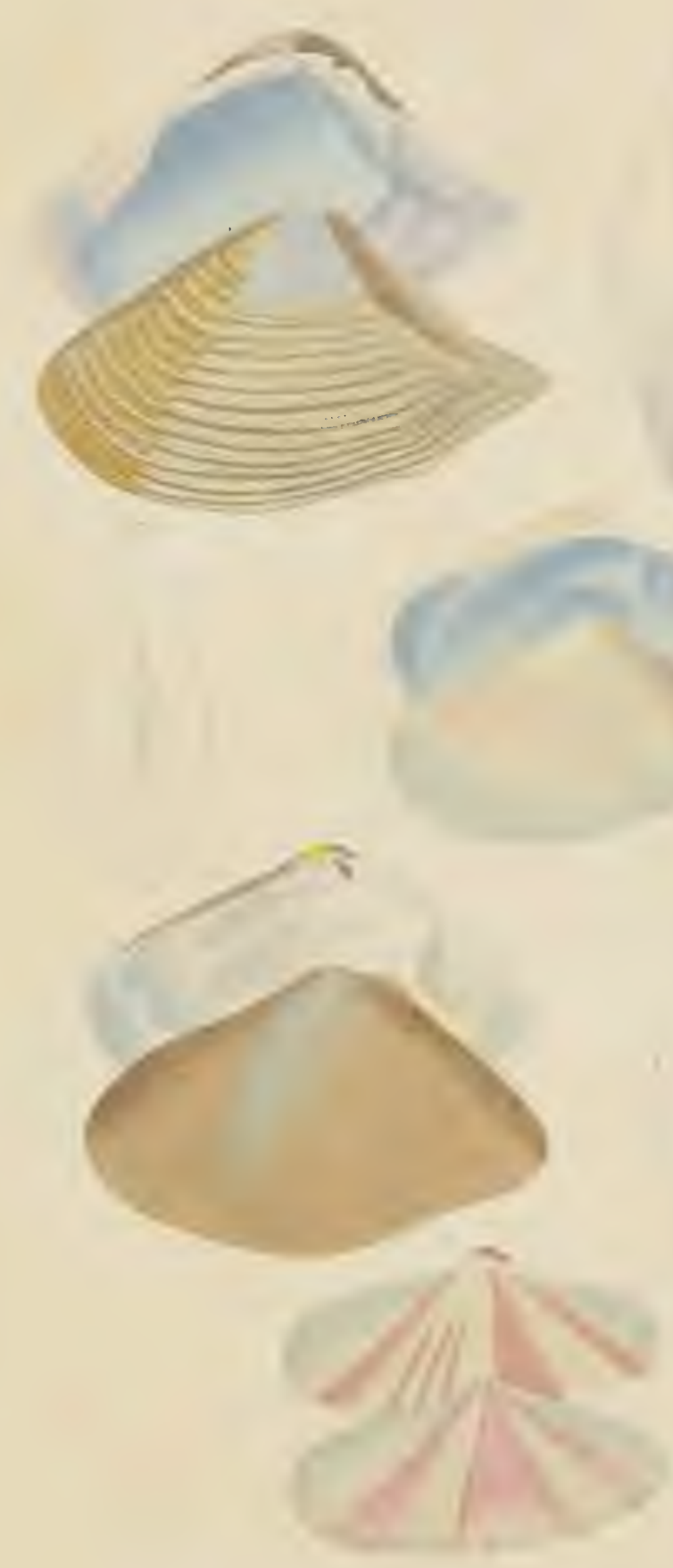

1. DONACl: bee de plute

2. D) I. IC des Canards.

S.C. Il'se du brésil

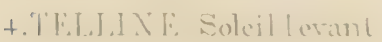




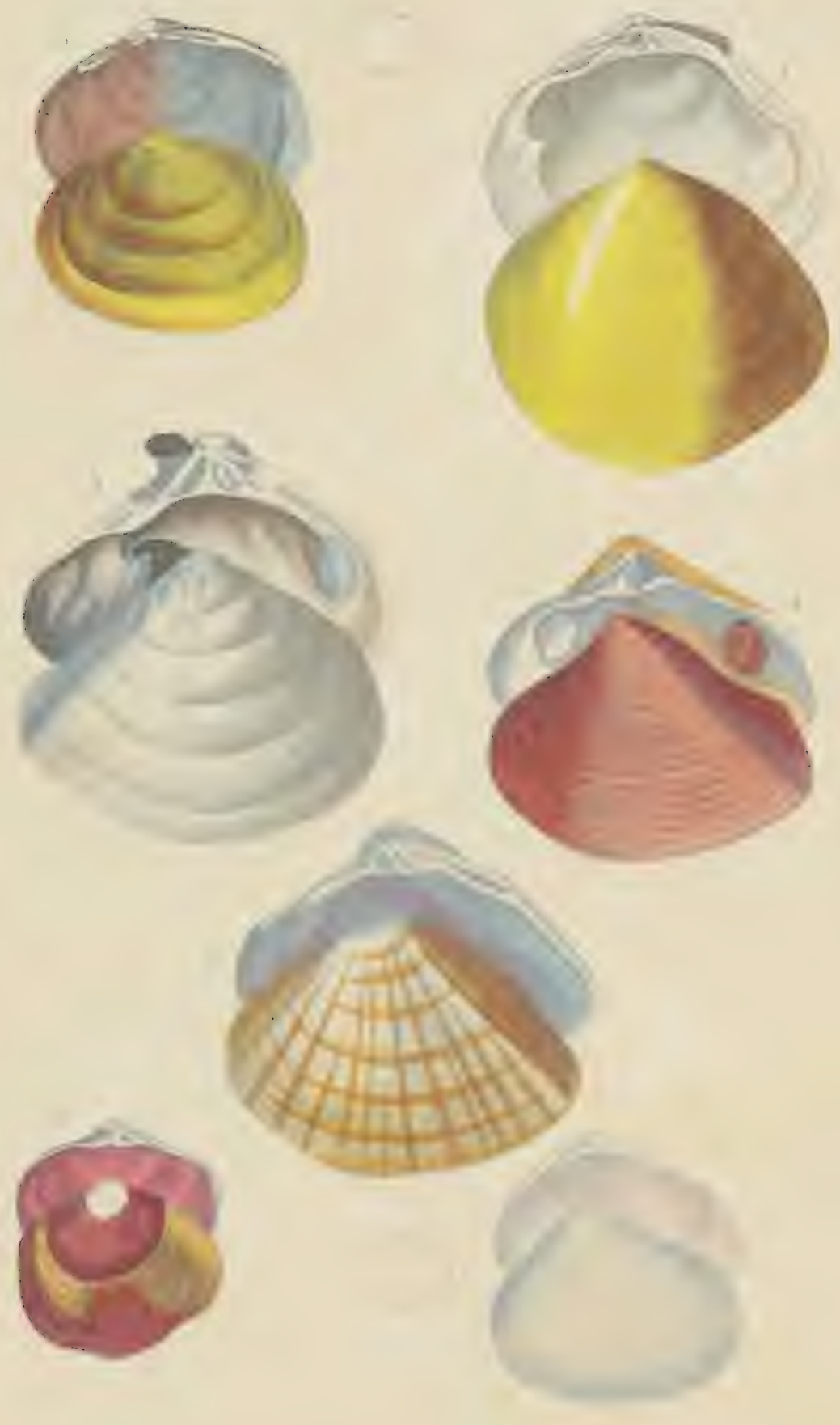

1. CYCLADI) comée. 2 CYCLADE de Cerlan.

3. GLITHÉL à ravons + CRLSSITELLE silloméc.

5. MACTRE lisor. 6 OXGULINE transterse.

7. ERYCISE cardioïde. 


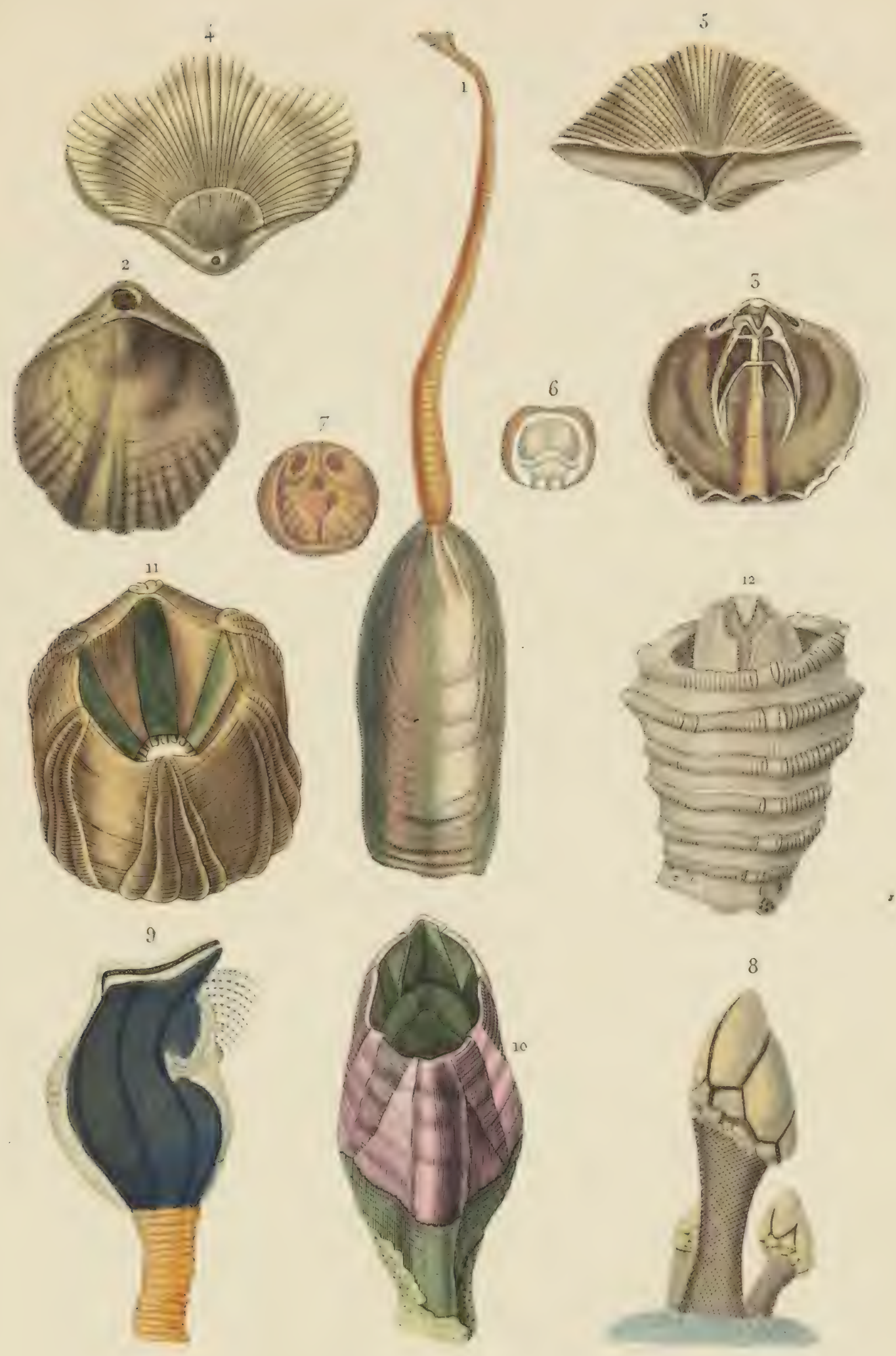

1. LINGUTE anatine.

7. CRANIE en masque.

2.5. TEREBRA'ULE dorsale. 8. POLLICIPEDF groupé

\begin{tabular}{l|l} 
4._ailée. & 9. CINERAS à bandes.
\end{tabular}

5:— canalifere. 10. BALANE tulipe

6. ORBICULE de Norwege. 11. CORONULE diadème.

12.TUBICINELLE des Baleines 


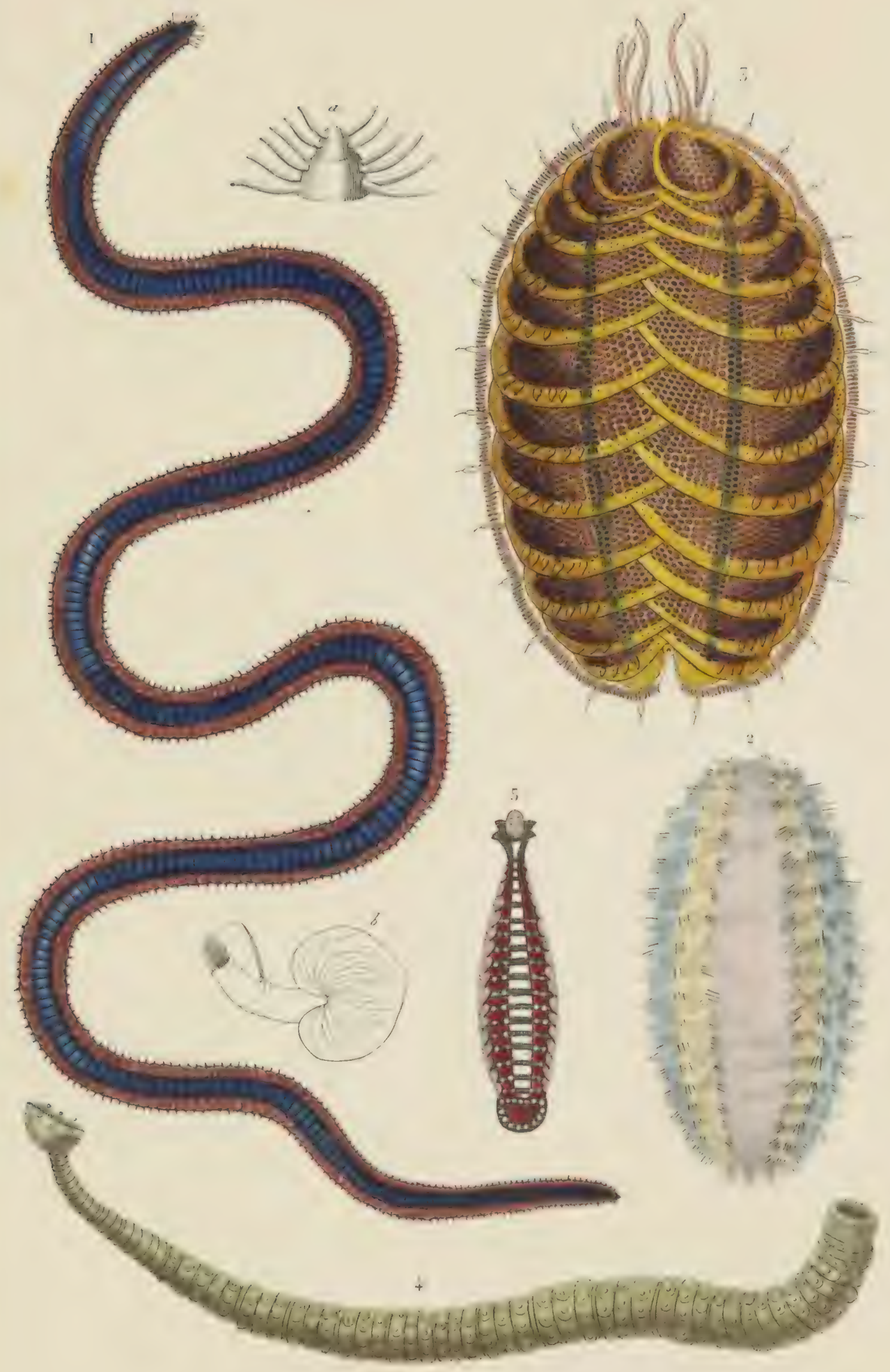

1.PHYLL OD OCE de paretto a b kete et pied gressis

2 APHRODITE hérissée

3.POTYNOE épineuse

4. PONTOBDEITLE épineuse.

5. CLEPSINE bioctitée.

Sunneres $S_{c}$ 


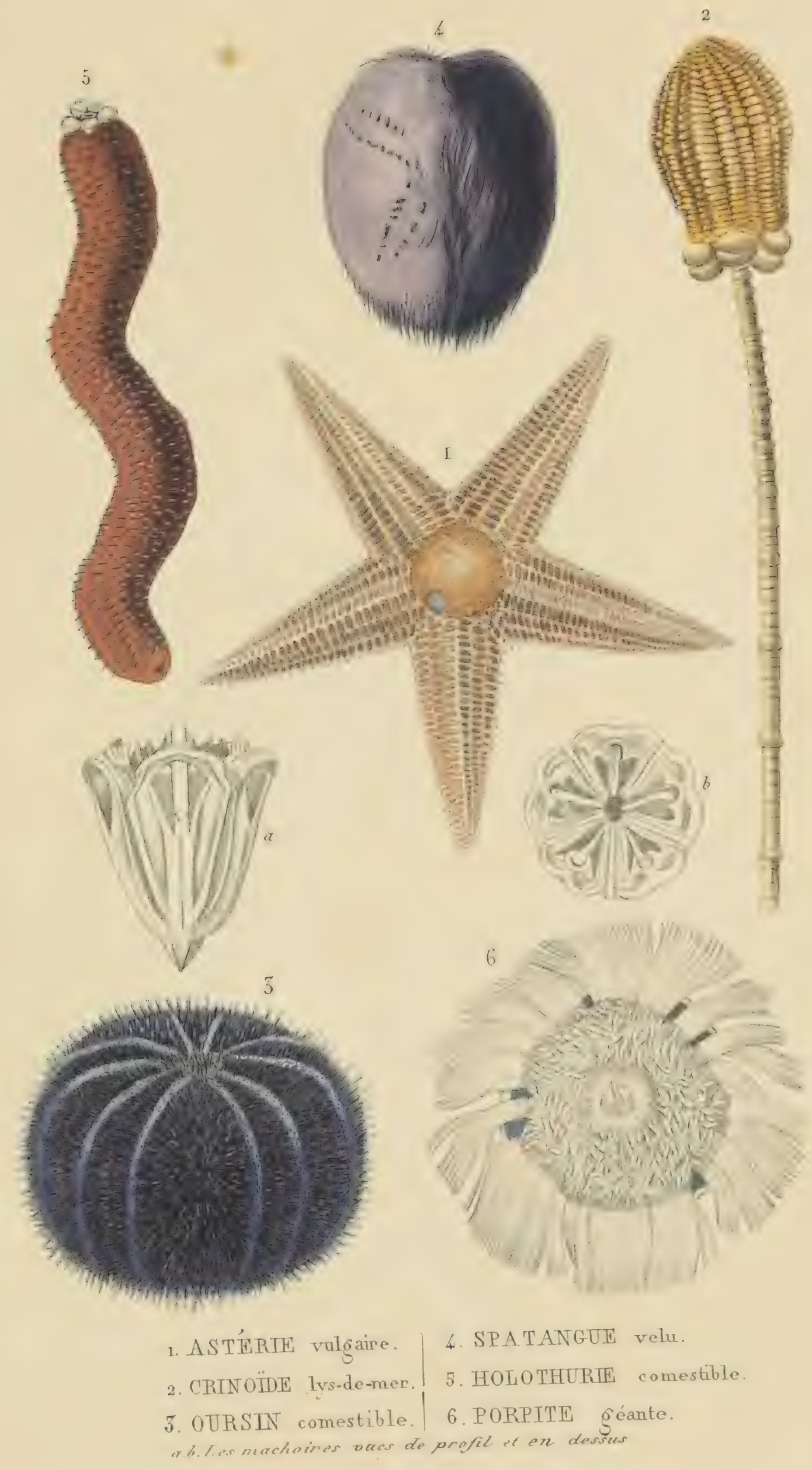


6
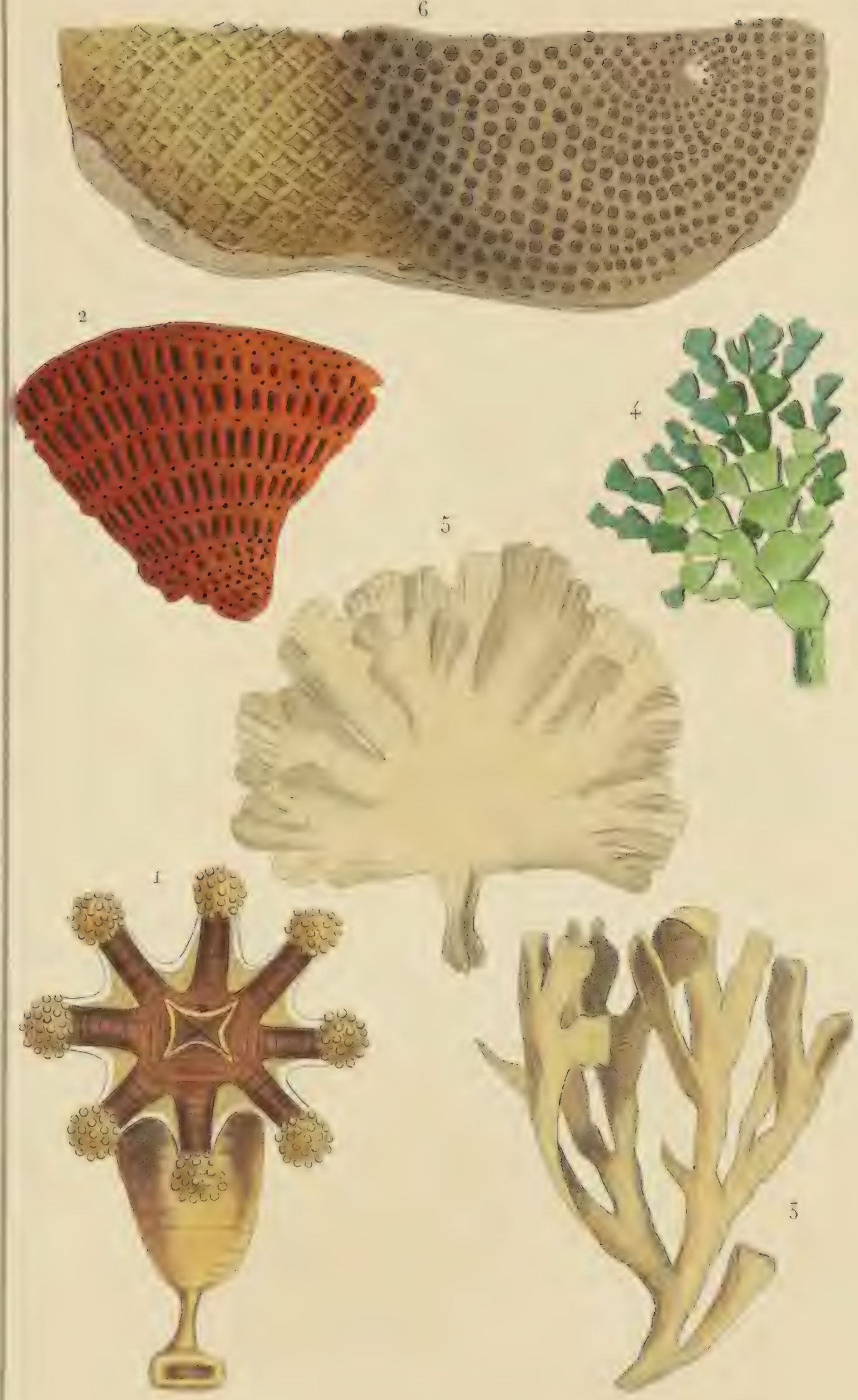

r. LUCERNATRE auricule. 4 4. FL ABELL AIRE raquette.

2. TUBIPORE pourpré. 5. UD OTÉE flabellée.

3. FIUSTRE foliacé. 6. RF́CFPTACULITE deneptune. 


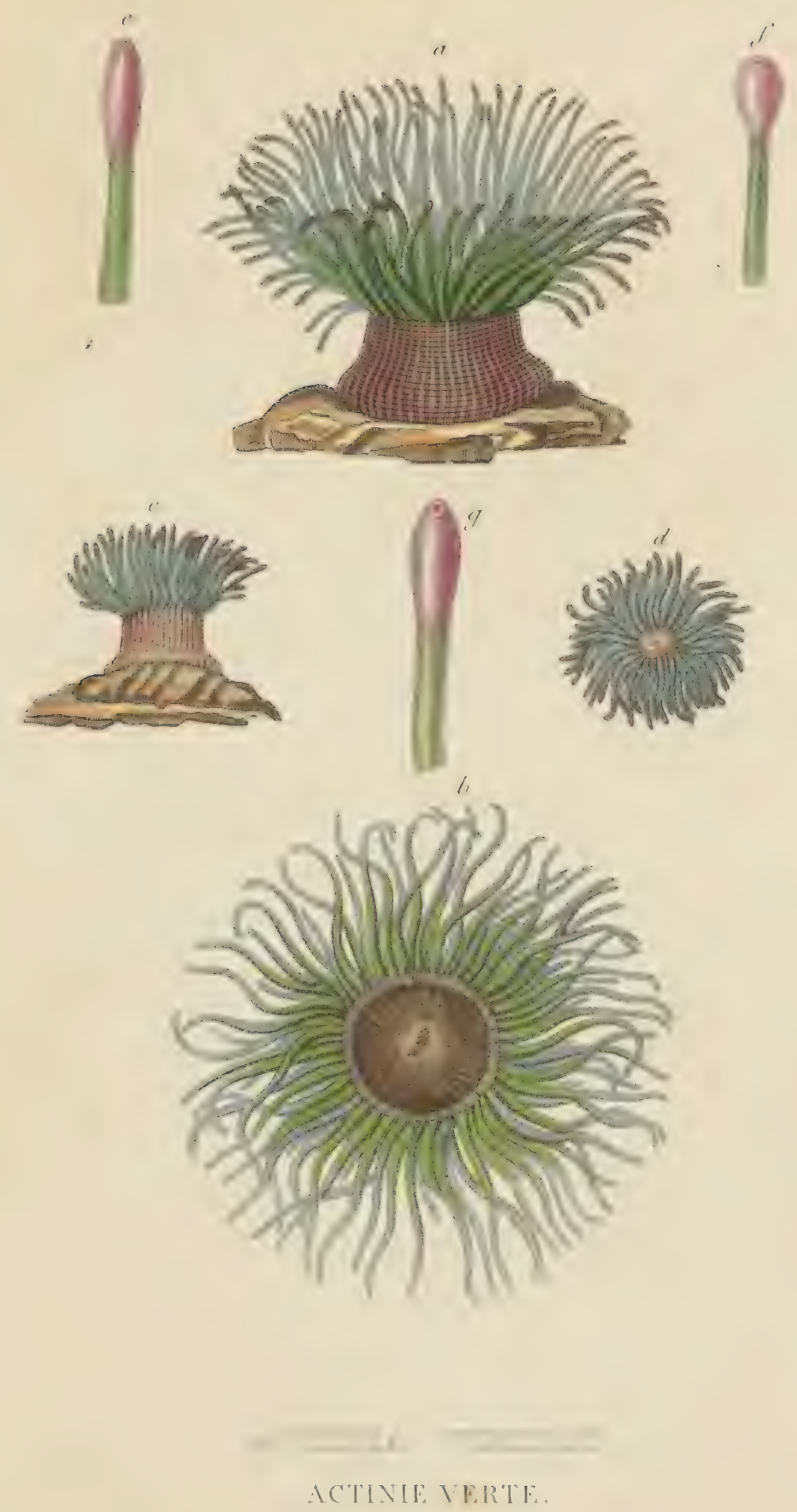




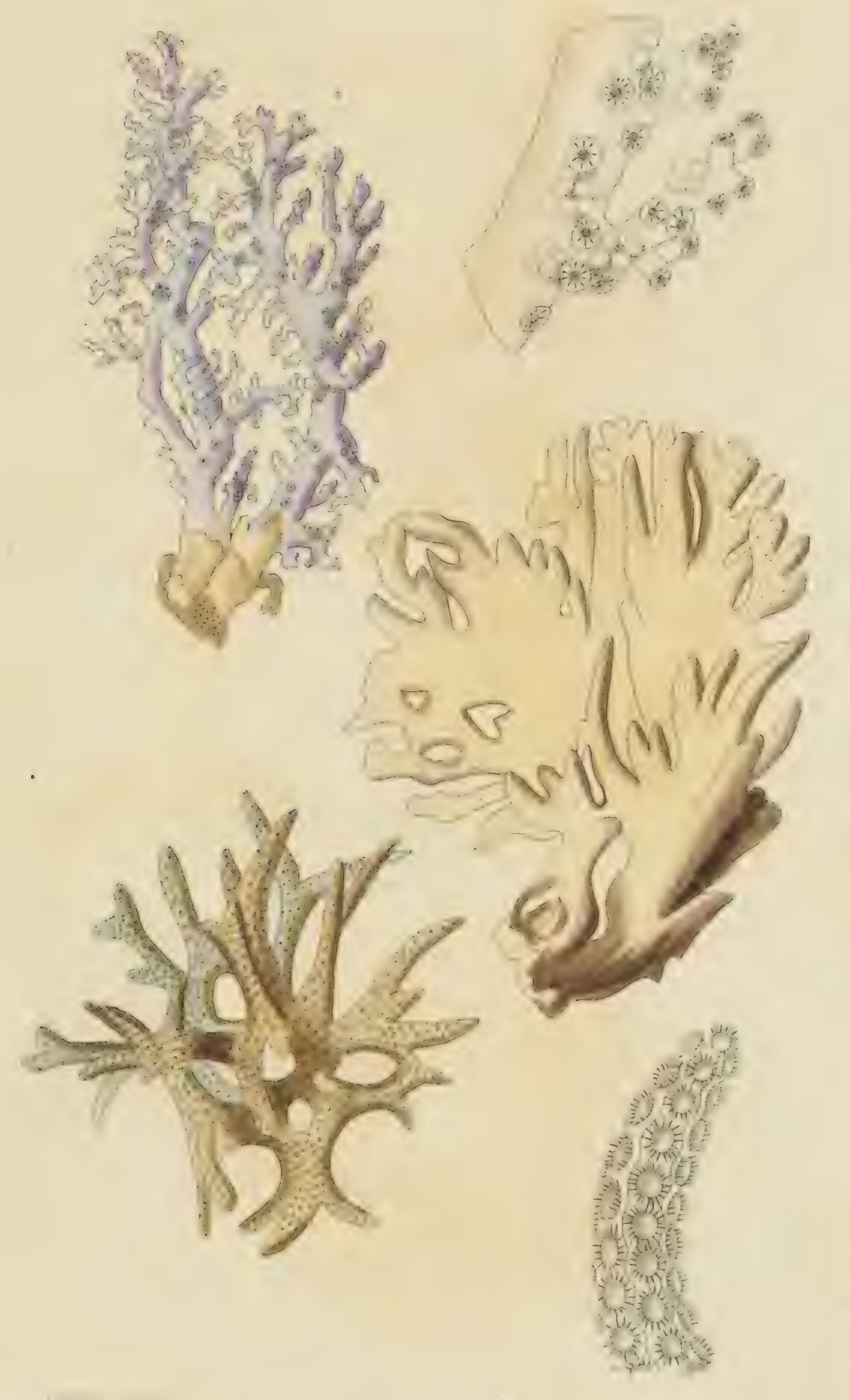

OCULTNF ROSE.

MITLEPORE CORNE DELAN .

SFRTATOPORE PIOUANT'. 


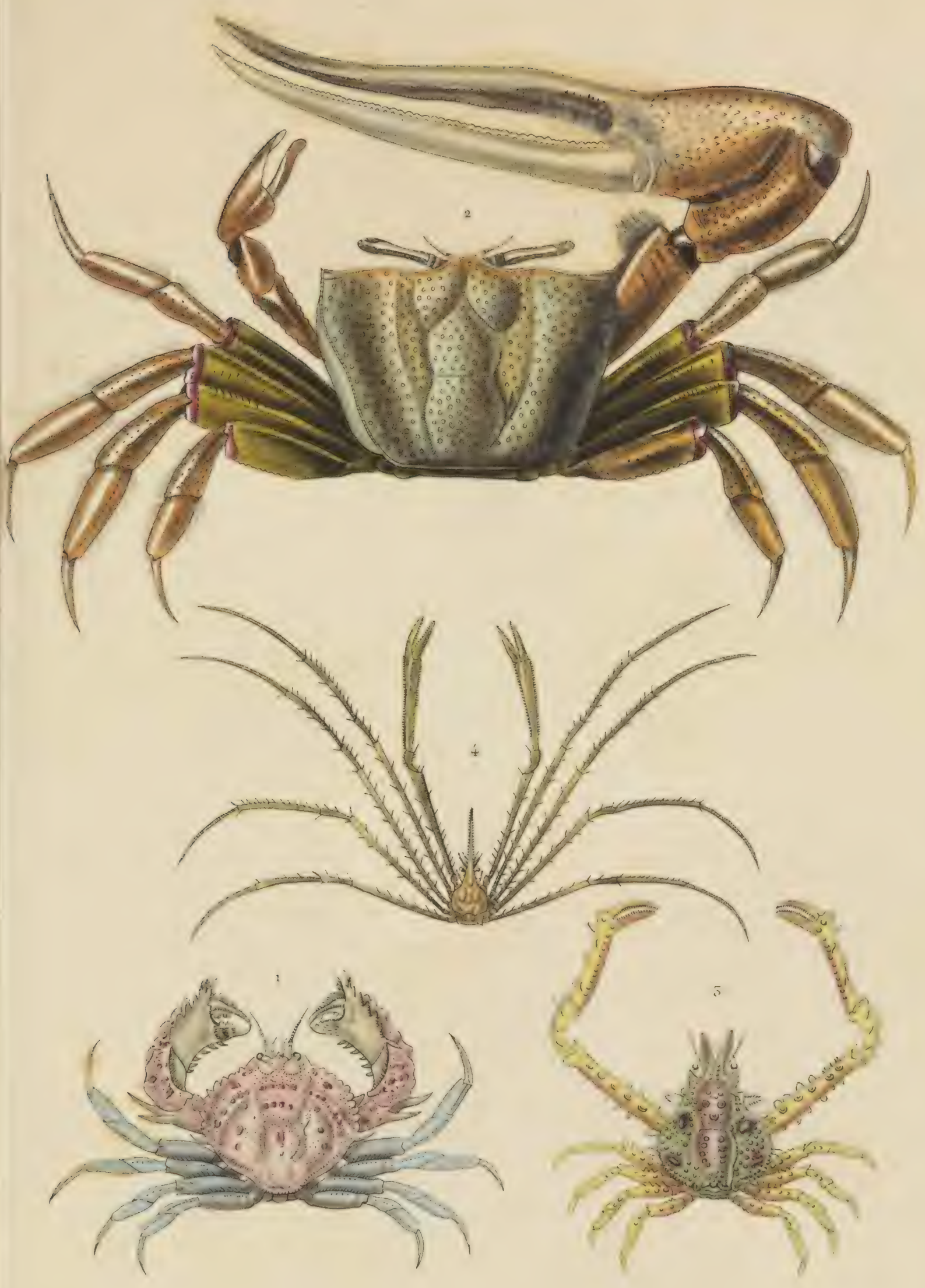

r MUTR STE MATNS-ENNCRETE Mursia cristatn

2 GELASIME DH TANGER Gelasimus tangeri

5 EURYNOME RUGUEUSE Eurrymome aspera.

千 STENORITYNQUE FAUCHEUR Stenorlignclus phalangium. 


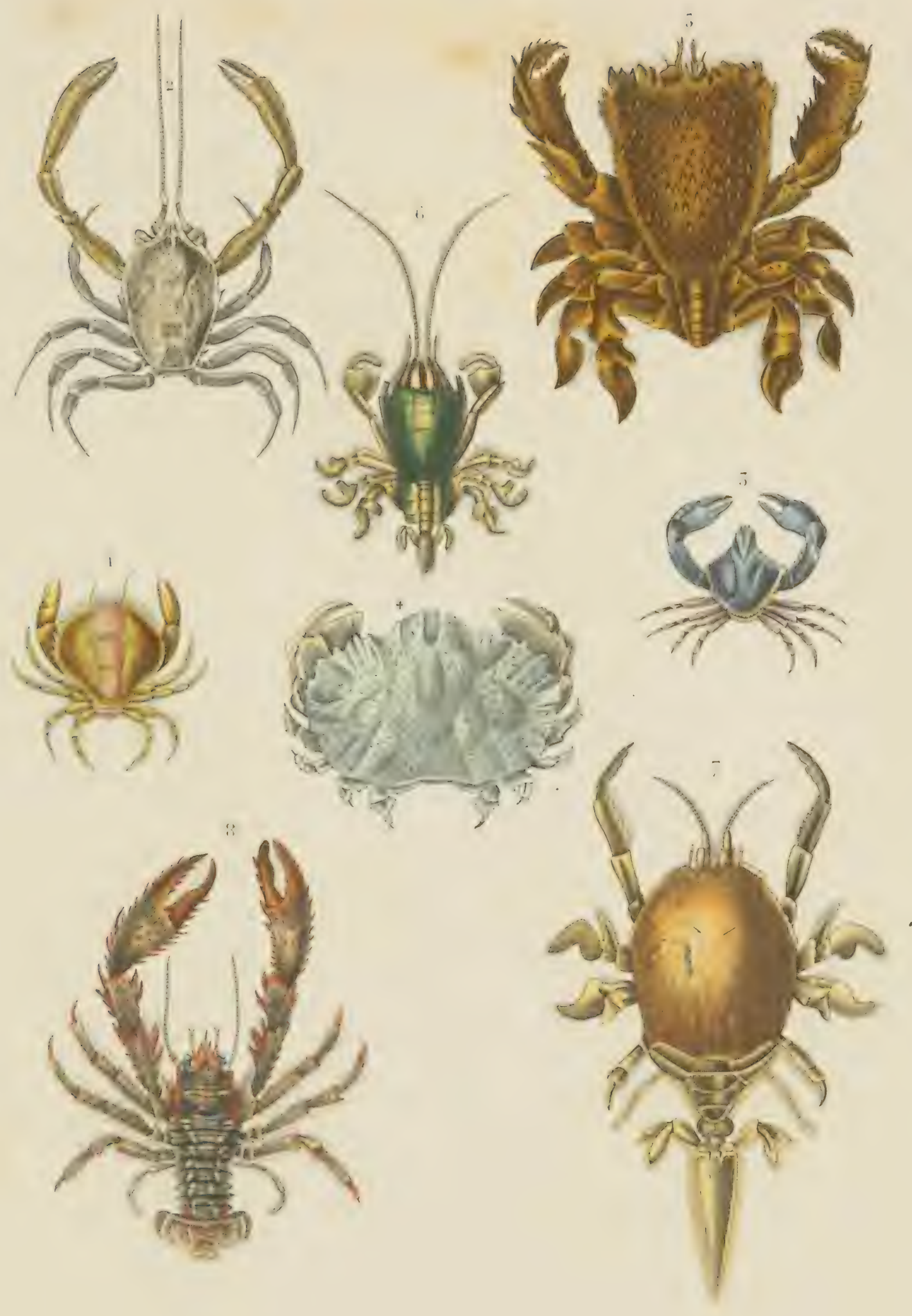

1.THTH luisante.

․ RANING dentée.

2. CORYSTE denté.

(i. HIPPE émérite.

3. T,EUCOSIF, craniolaire.

T.REMIPFiD tortue.

4.A'THRA rabotetx.

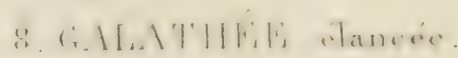




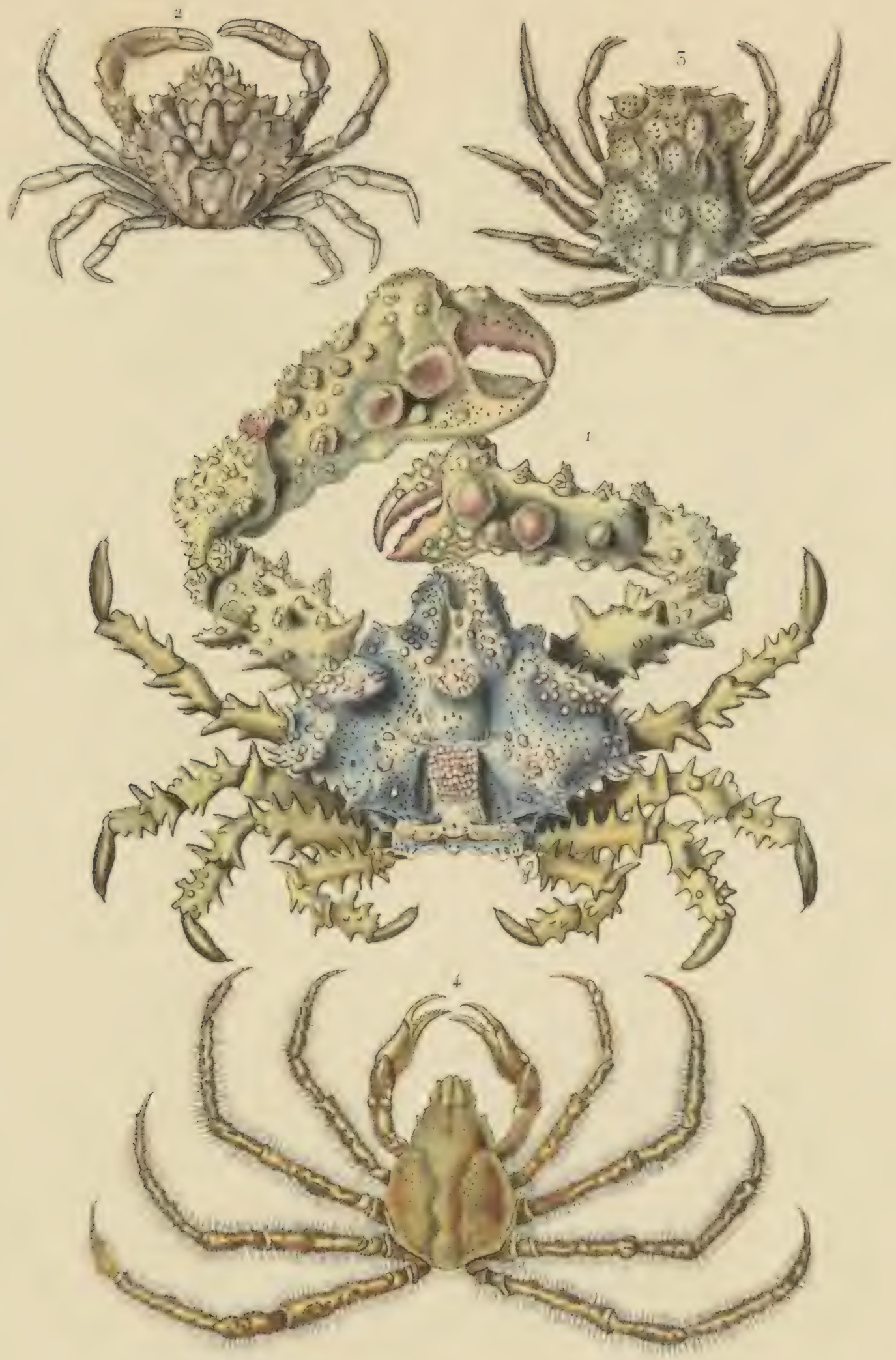

1. PARTHFXYPE ITORRTBTit. Parthenope horrida.

2. MITHRAX A BORTIS FiPINEUX. Mithrax spinicinetus.

7. MICTPPH PHYJTRE. Micippa phylira.

†. CAMPOSCIF. Cimposcia retuja. 

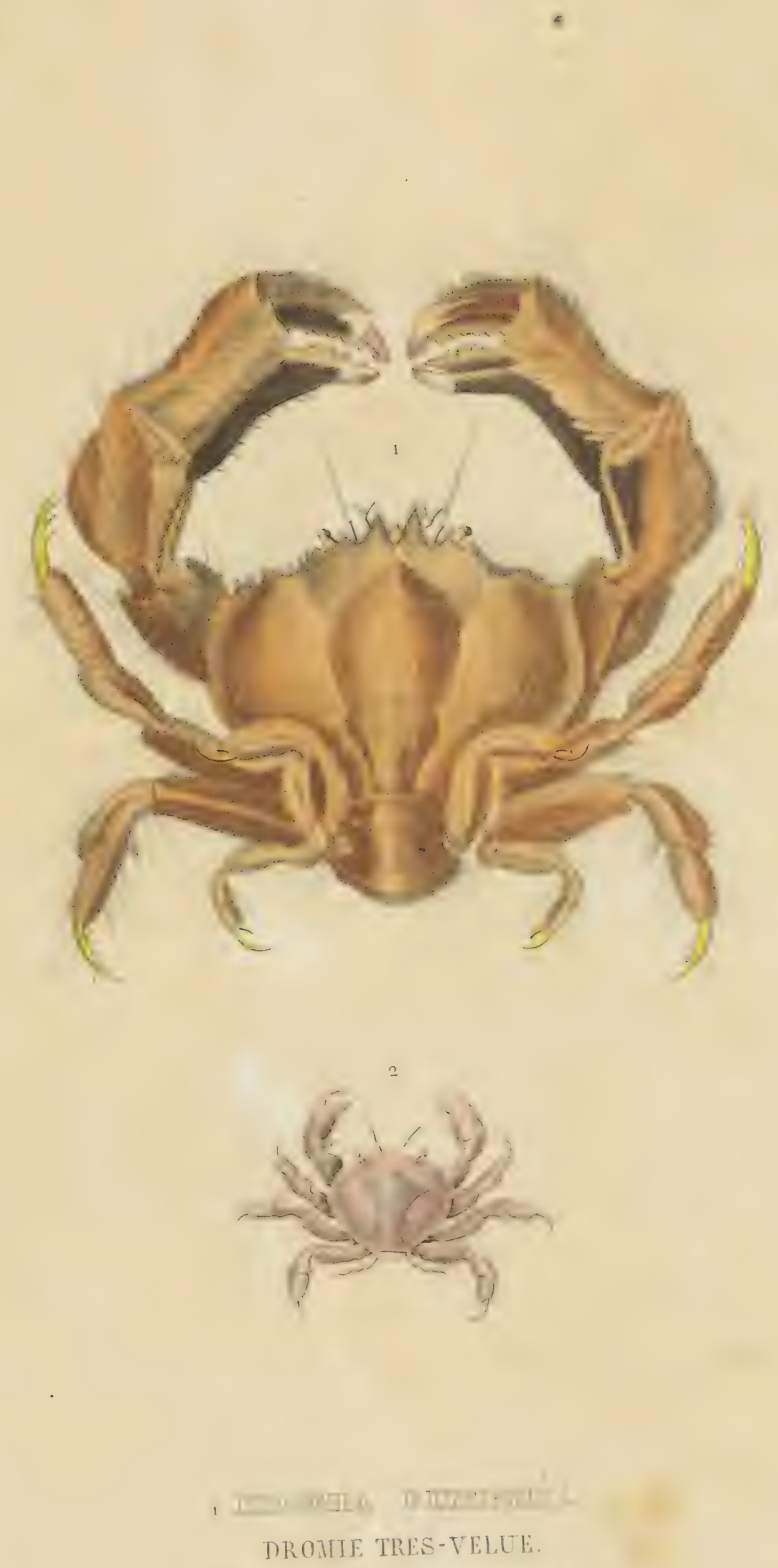

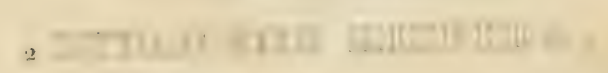

DTXANENE HISPIDE. 


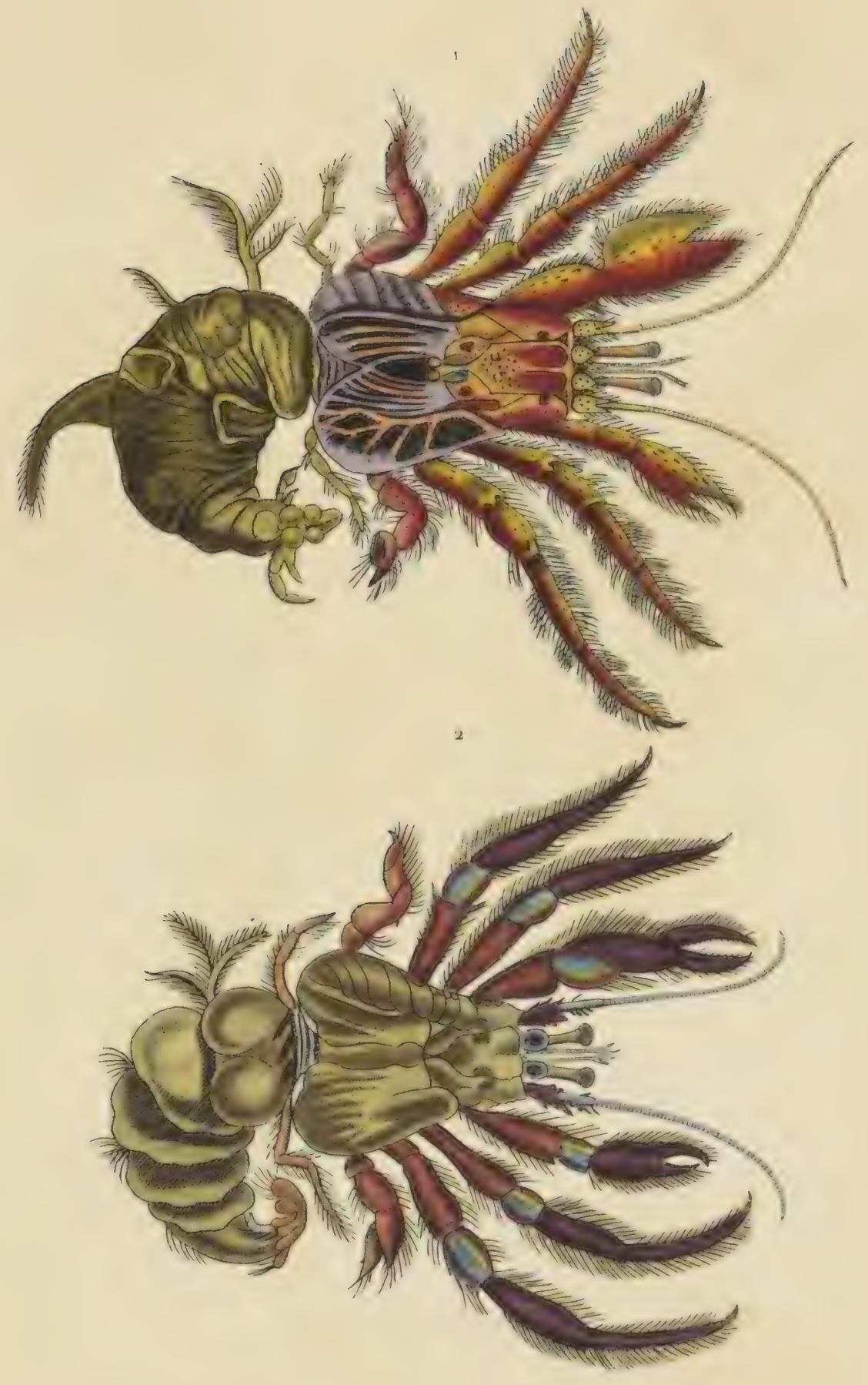

1. IPAGTIRUSS SANGTUINOILIEIVITUS"

PAGURE SANGUINOLENT.

2. IPAGUIRUSS GTITA.ITUS.

PAGURE MOUCHETÉ. 


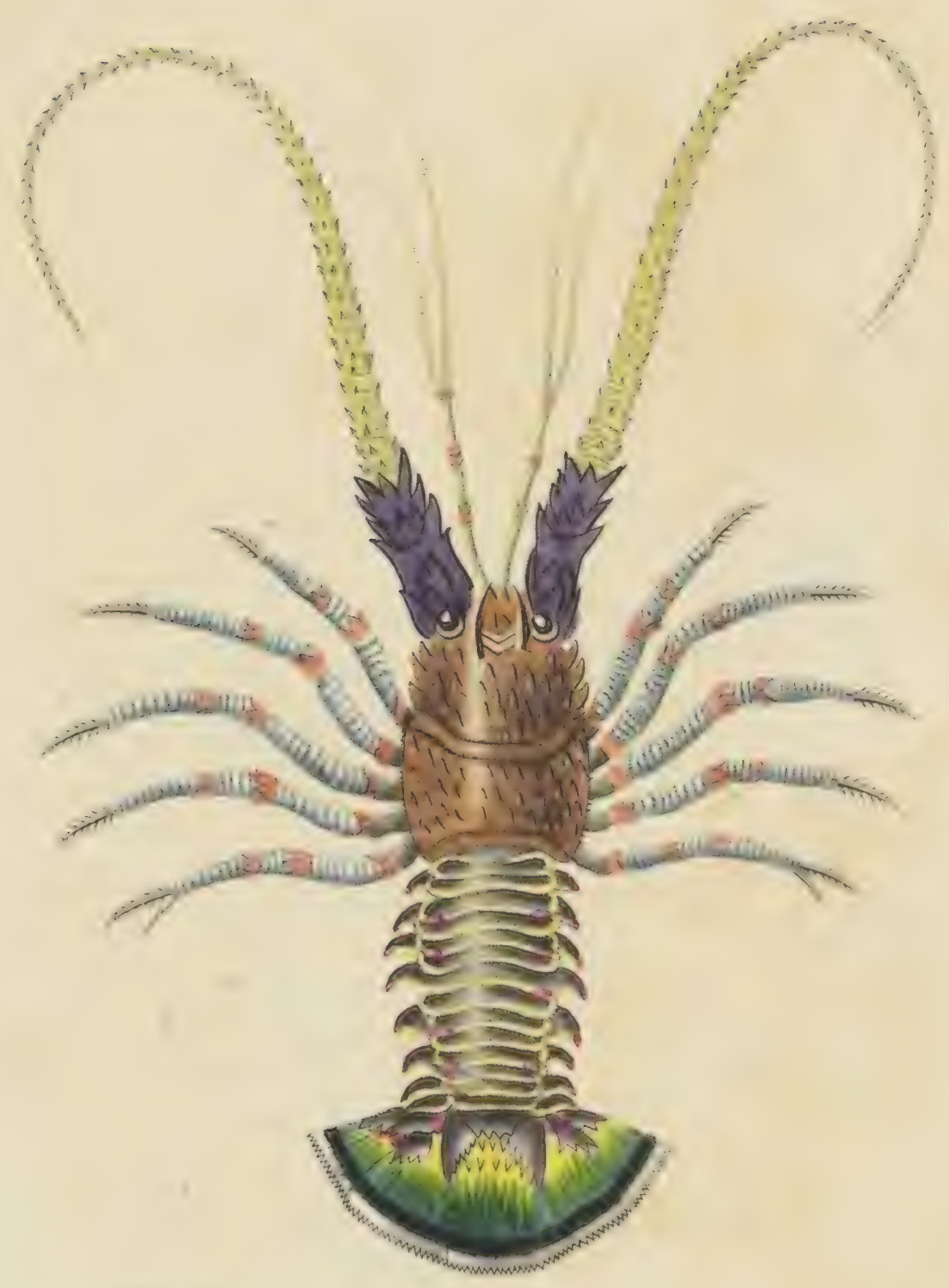

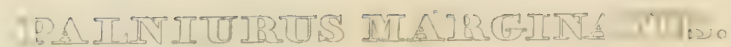

I.ANGOTISTW BORDTET 


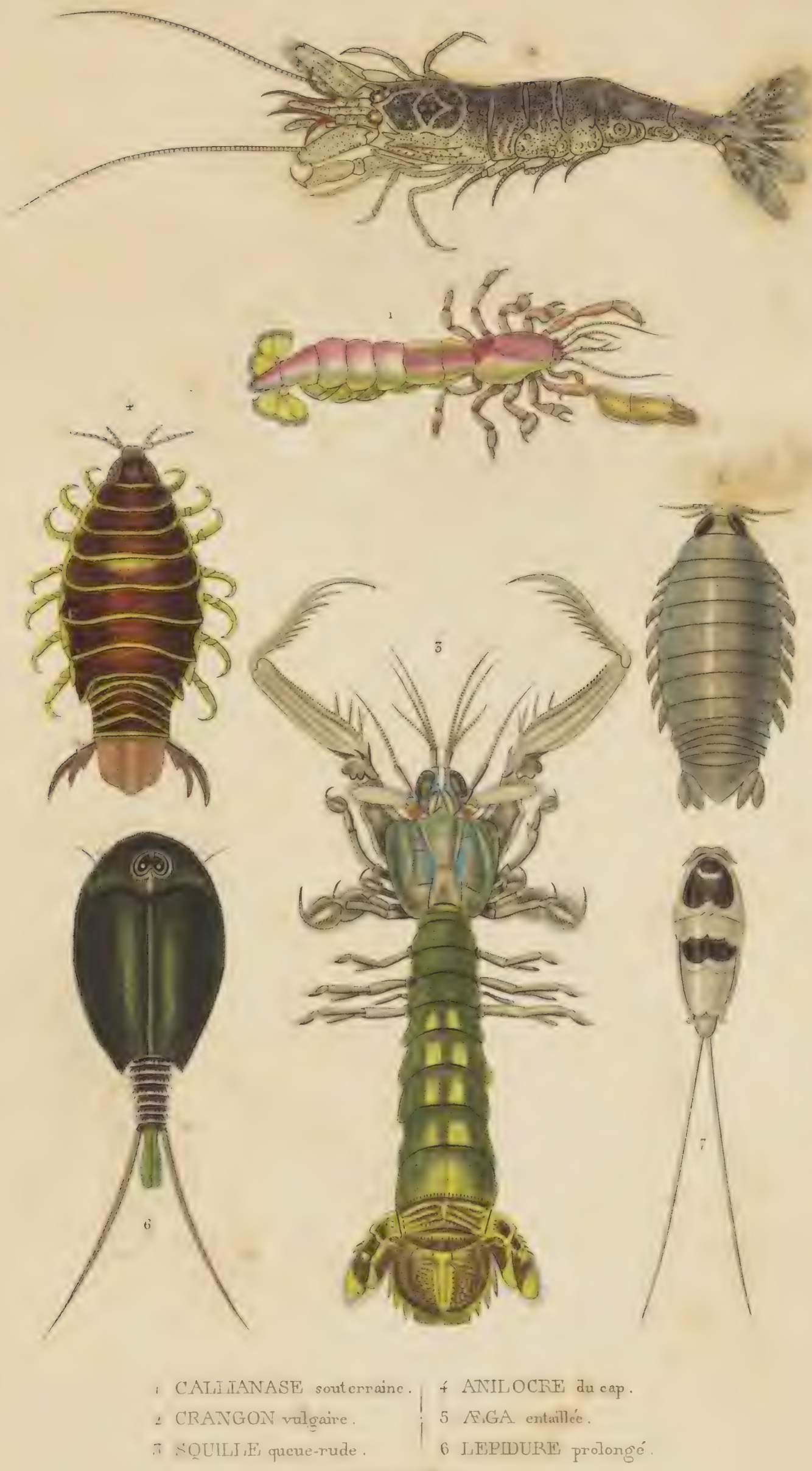

7 PANDARE hicolore 


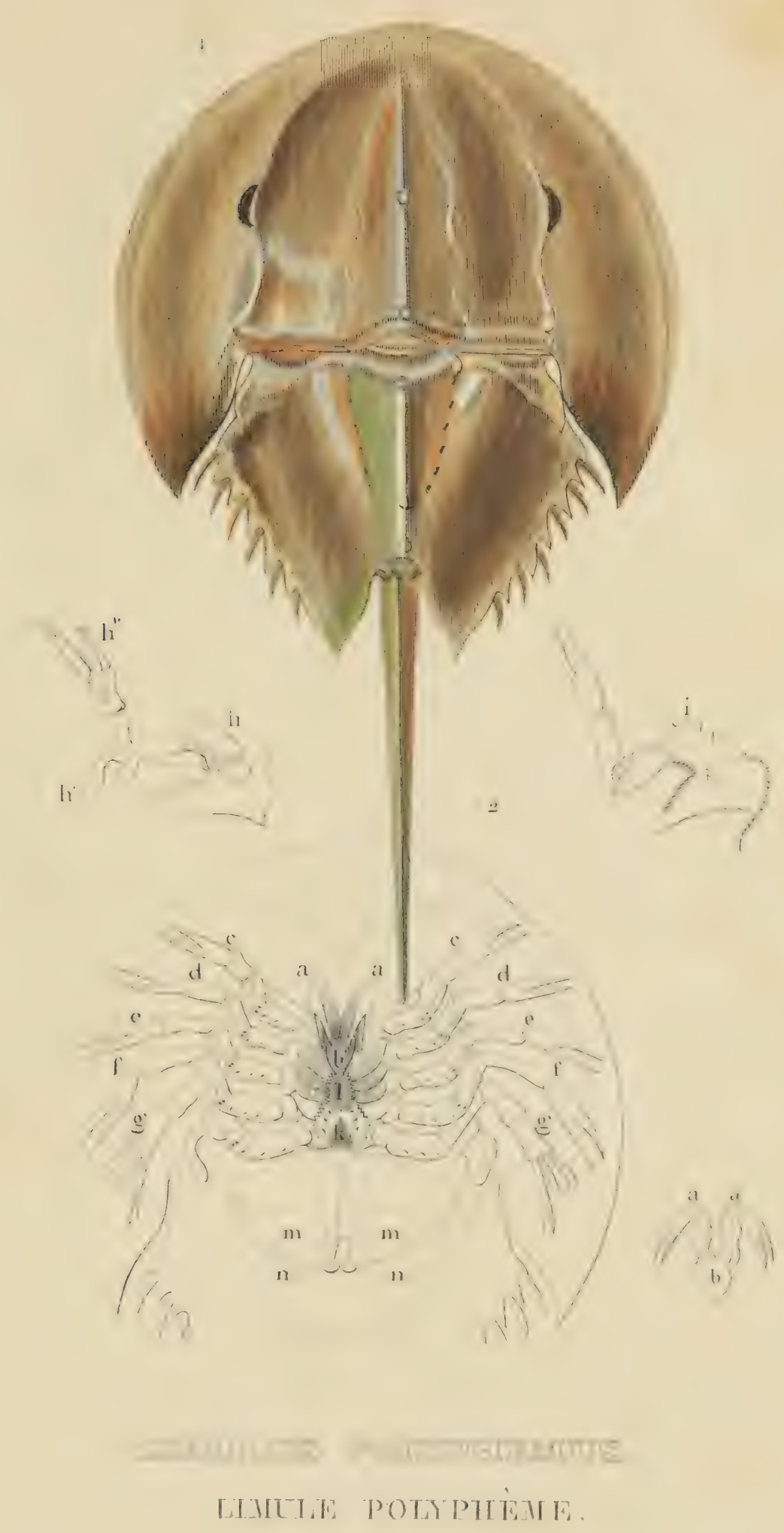




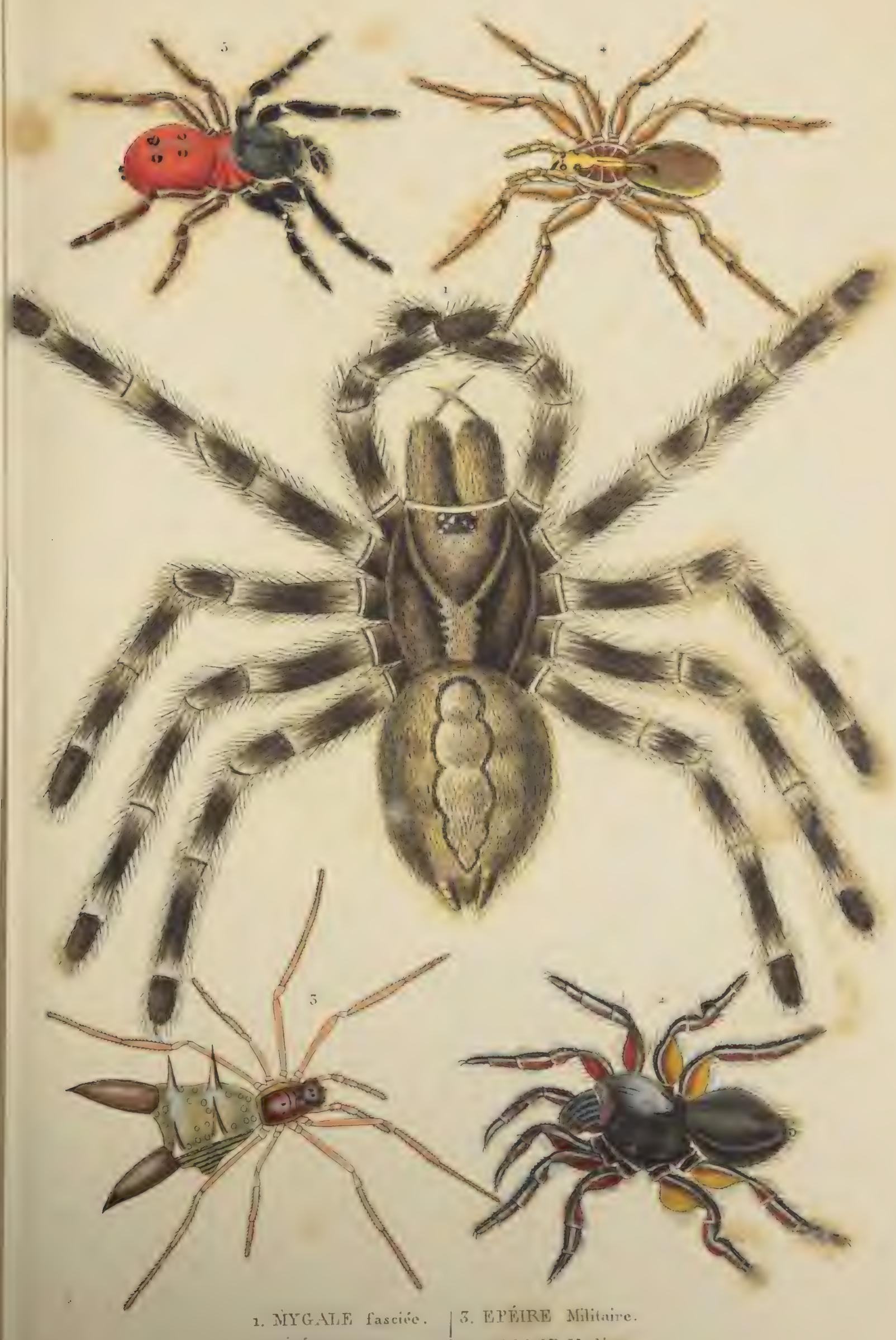

2. C'TENIZE ("hassiur. f. ATCOSE Xolline.

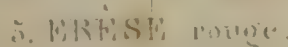




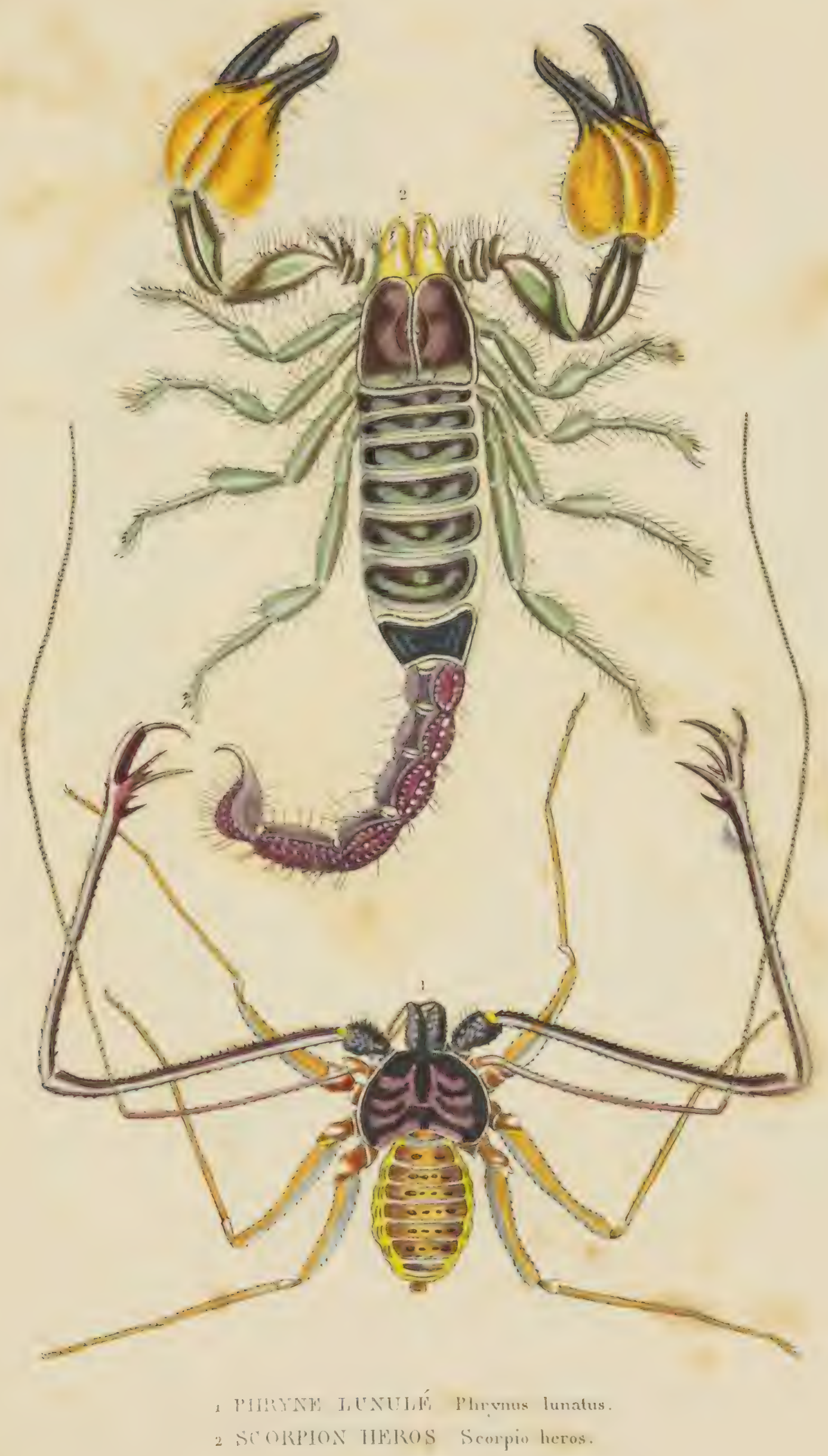




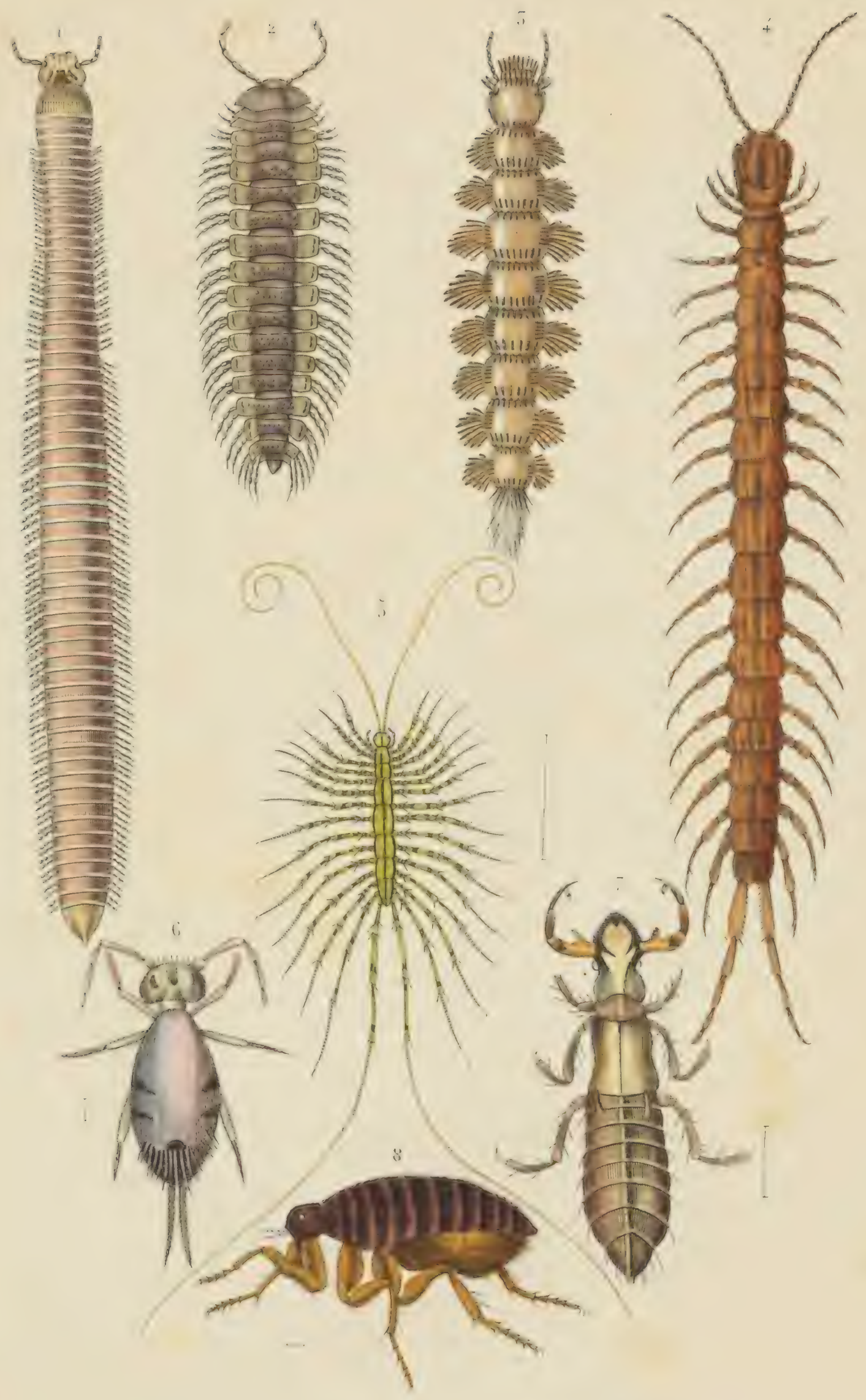

\footnotetext{
1. TUIE trés grand.

2. POIYDFinE granuleux.

3. POLLYXFNG lañure | FPHICOPTLRE de latbatros.

4. SCOAOPF.NDRli' mondante. 8. PUCF irritante
} 

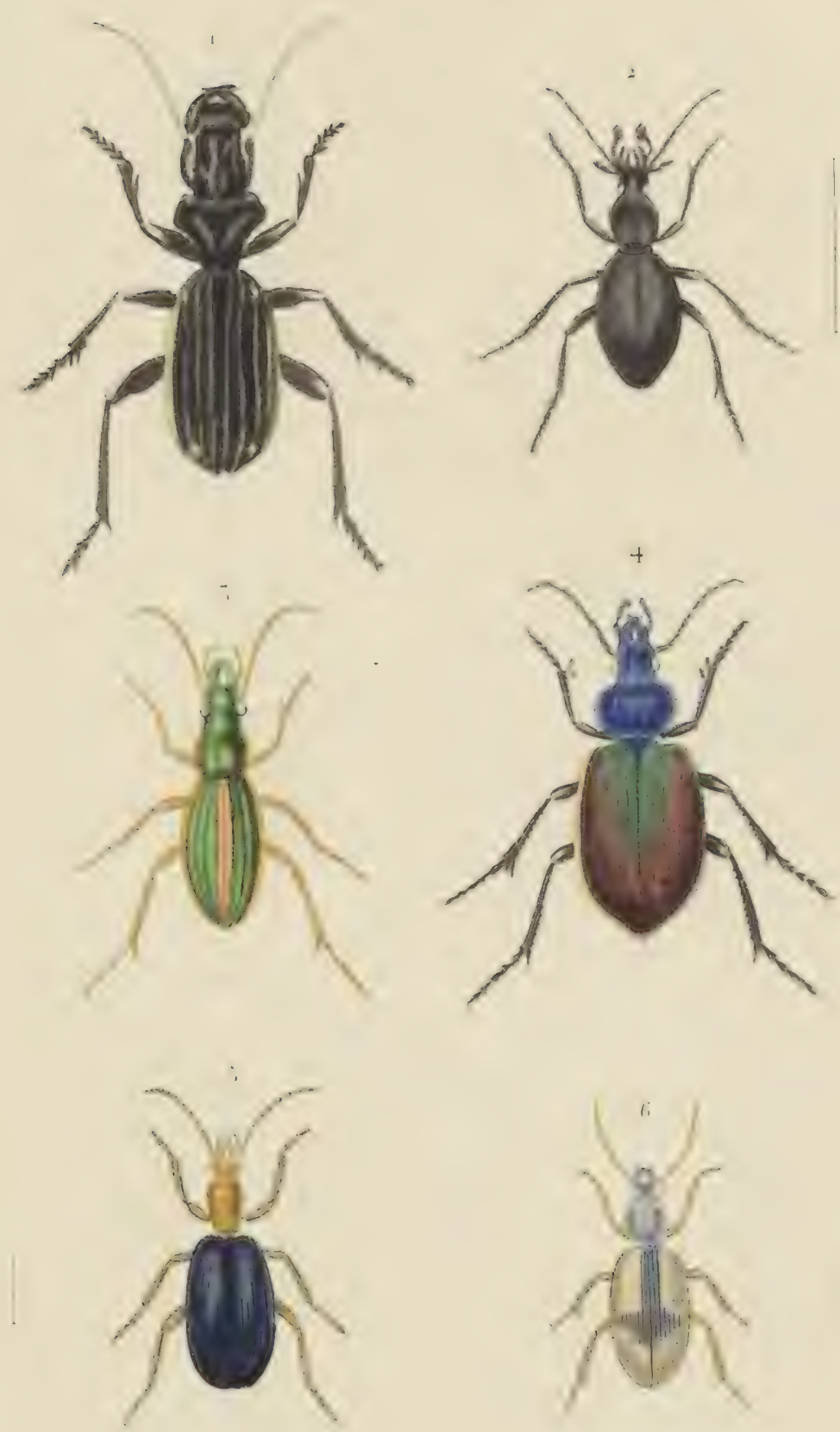

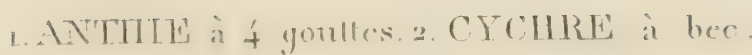

¿TACATHE doré. 4. CALOSOALE sycophate.

5TBRACHYNE pélade 6. BEMIBIDLON a quontles. 


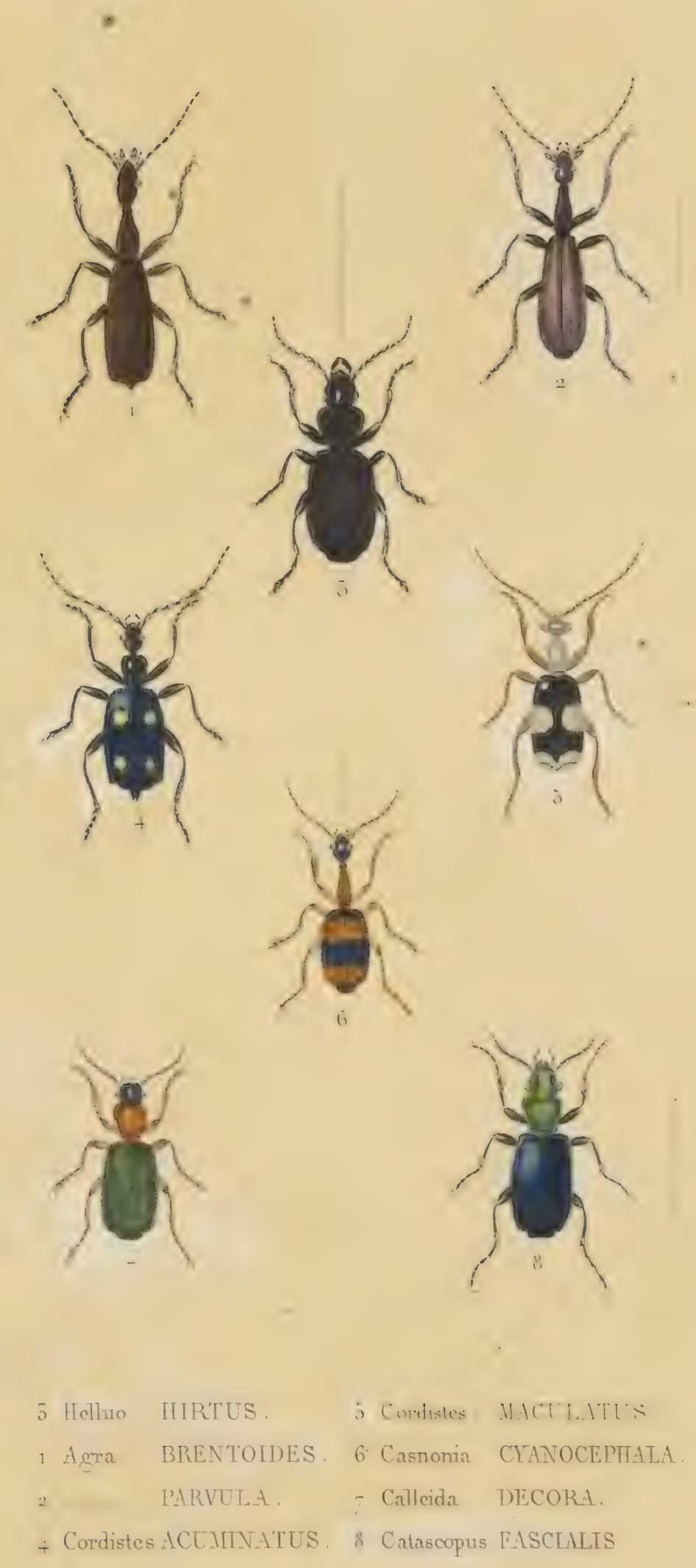




$$
N_{1}
$$



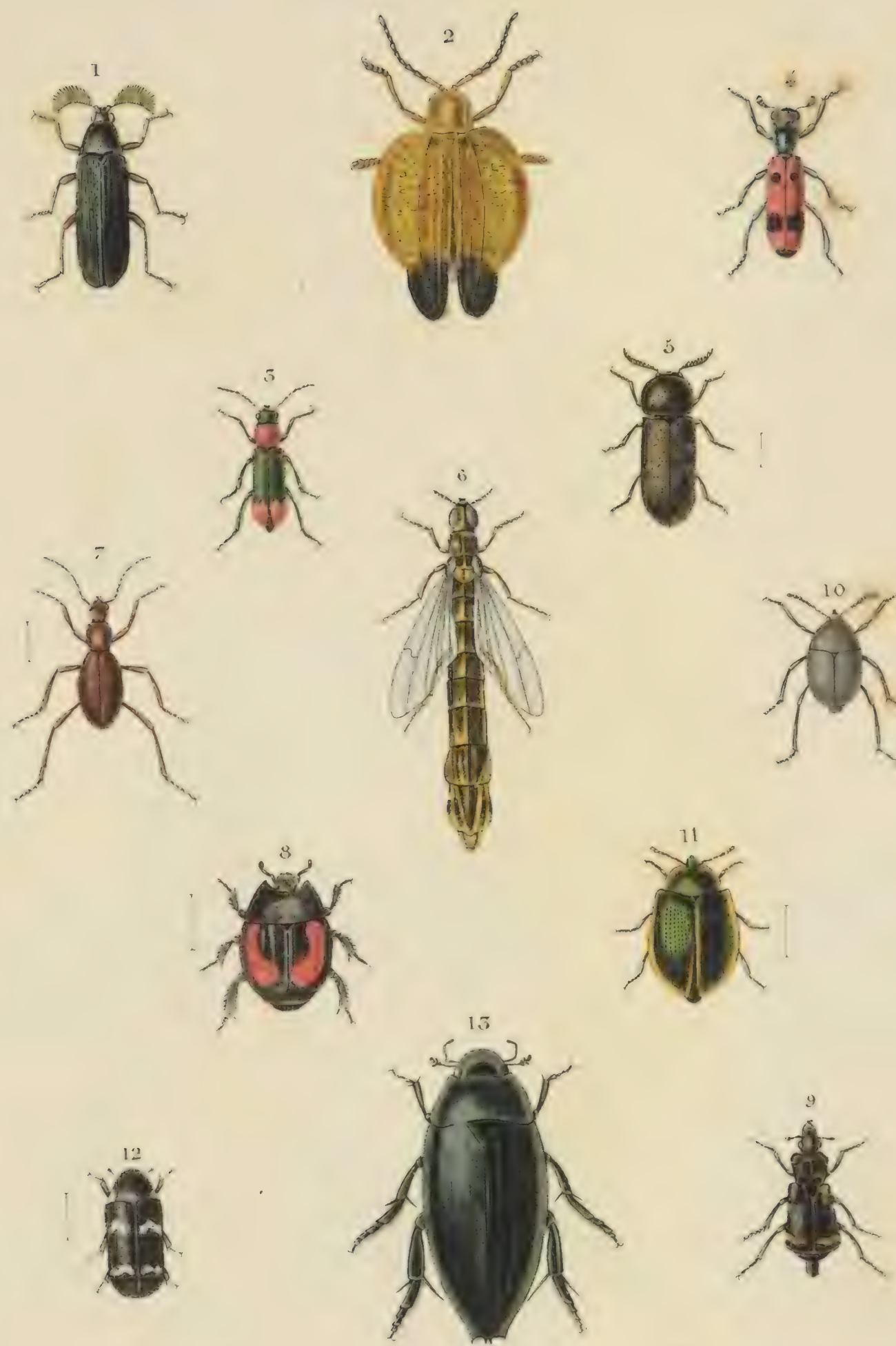

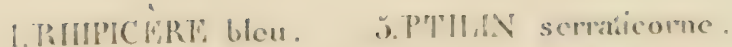

9. NF'CROPHORL marilime.

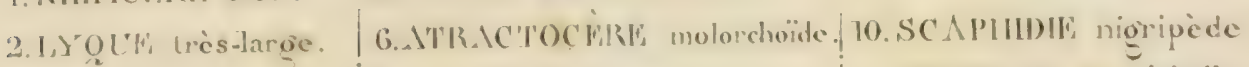

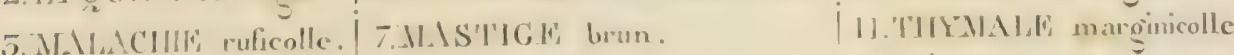

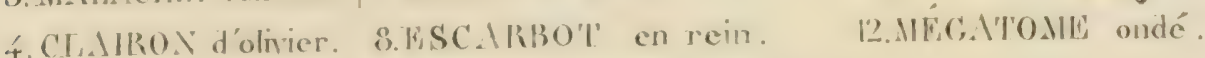
15. HYDROPIIIII, spinipenne. 


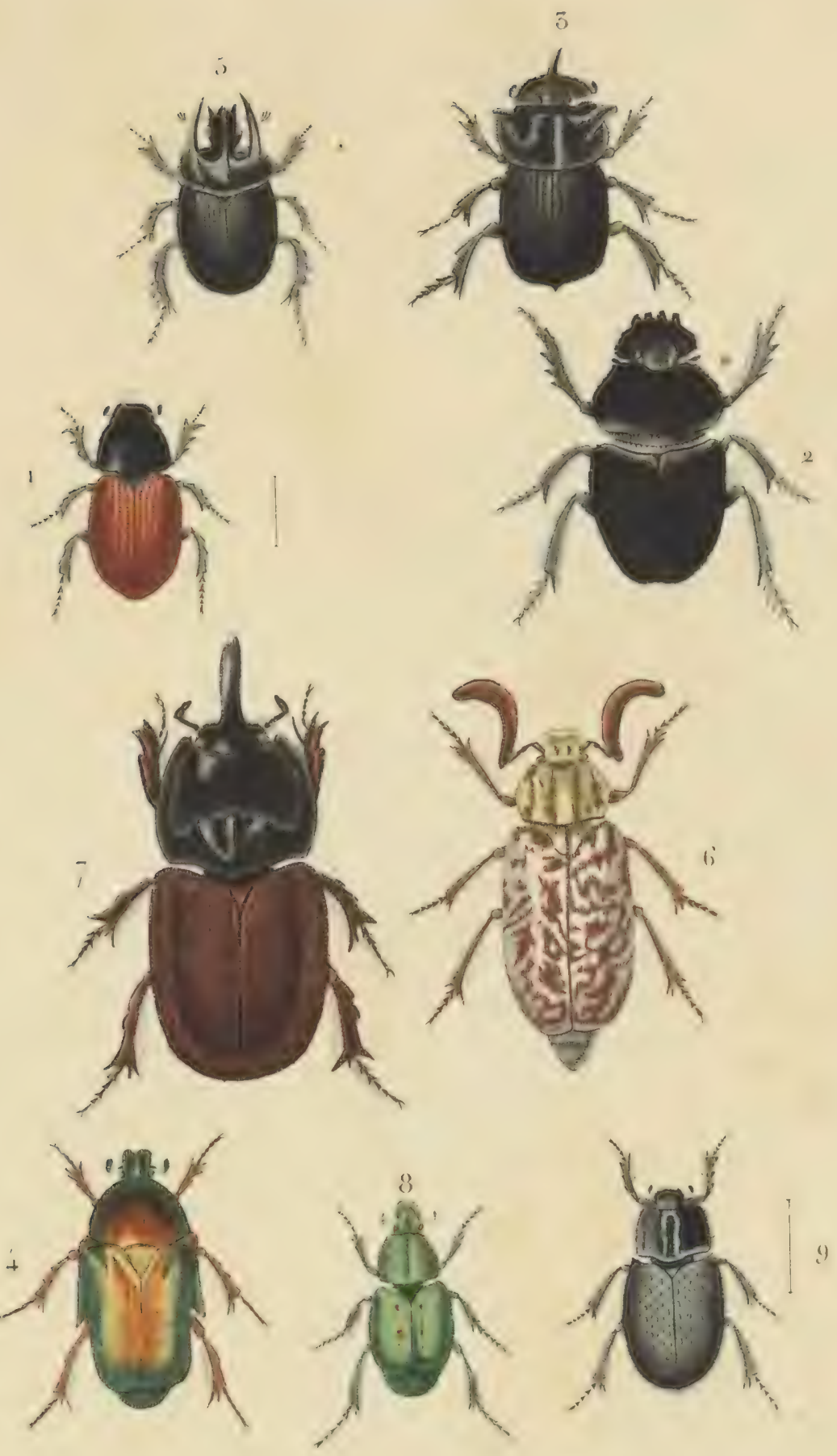

\footnotetext{
1. APHODIE simetaire 5. GEOTREP' phalangaste

2. ATELCHE sacre. 6. HANNETON toulon.

5. BOtSIFR lunaire

-. ORYCLES nasicone

4. CFTOINE métallique .. 'TRICHIE noụle'
} 


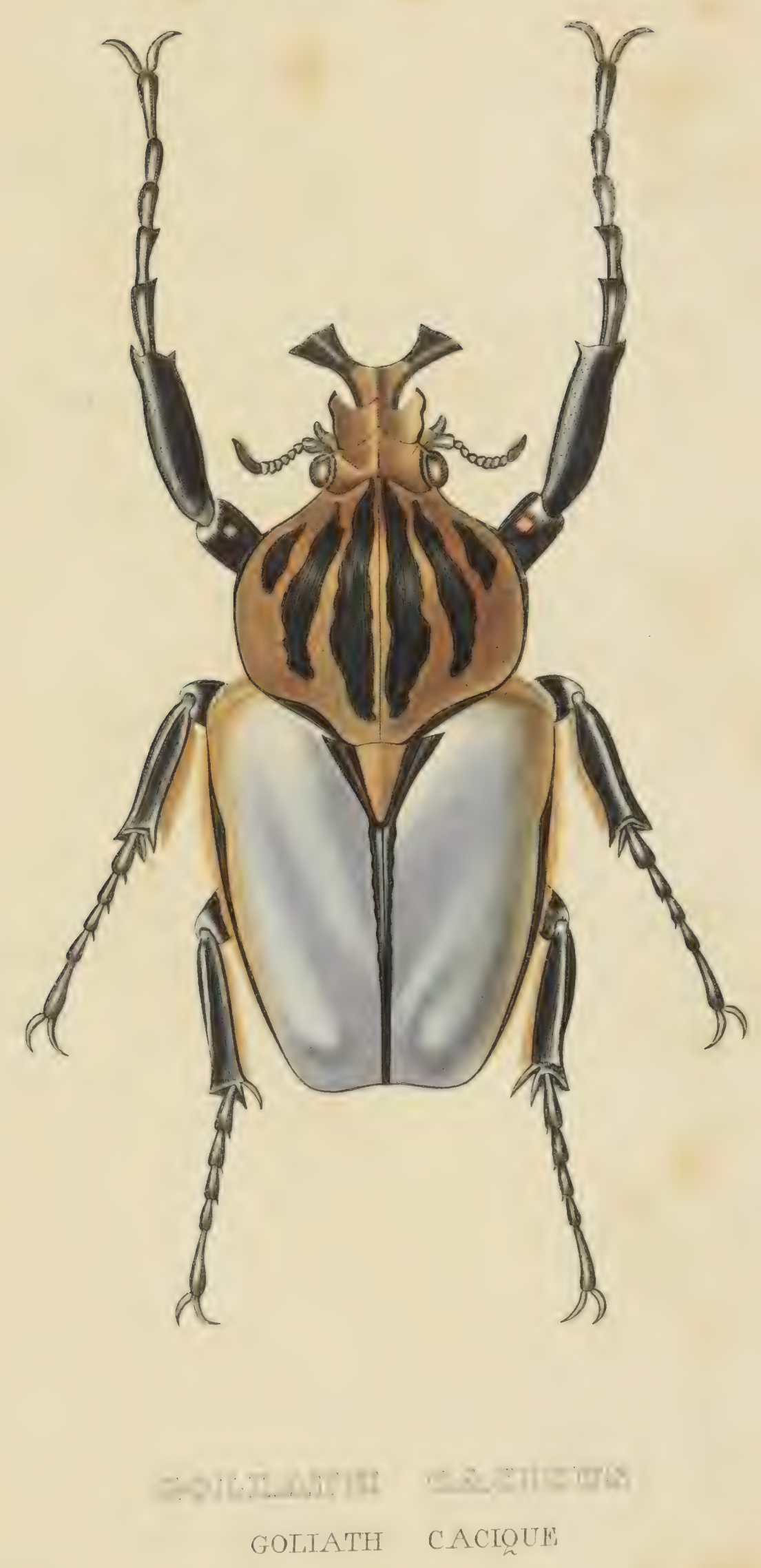




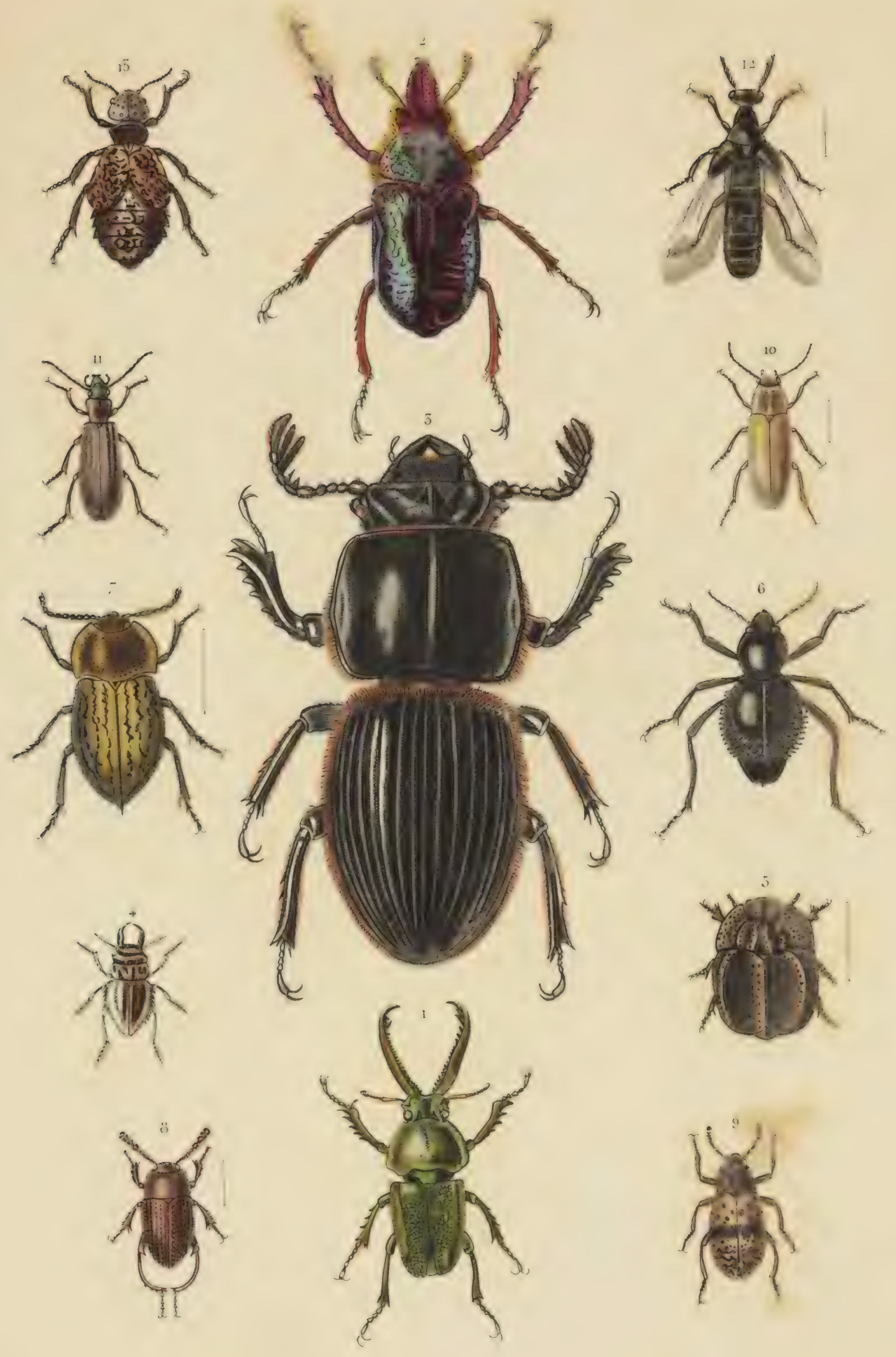

1. SPHÉNOGNATHE prionoide.

5. STEIRE à côtes. 9. SPHÉNISQUE peint.

2. PHOLIDOTE de humboldt. 6 MOLURE de pierret. 10. HALLOMFiNE humérale.

5. PASSALE de Gory.

7. OPATRE gris. 11. NY'IILE lisse.

4. CALOGNATHE de cherrolat. 8. LEIODE canclle. 12. MYYODITE américexin. 15. MÉLOÉ d'olivier. 

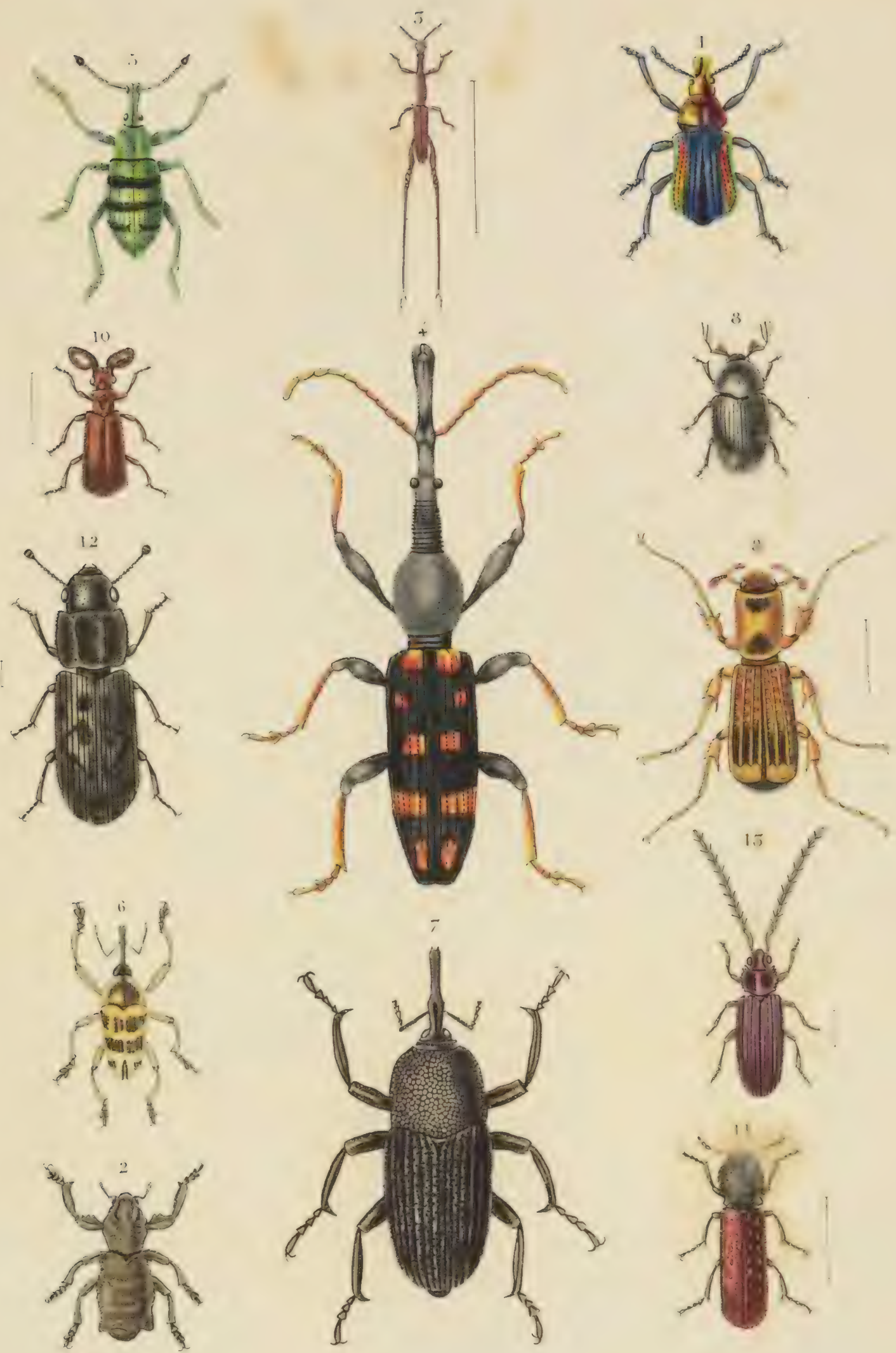

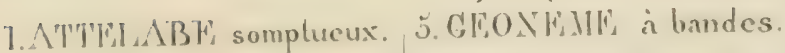

9. THSSEROCRRE remarquable. 2. ITFRPlis pipa.

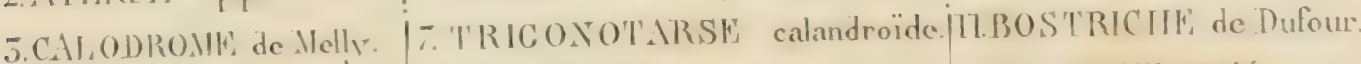

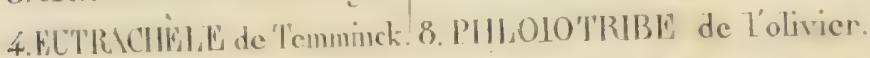
72.SYXeIIIT' ondé.

15. CUCLJE a paltes jaunes. 

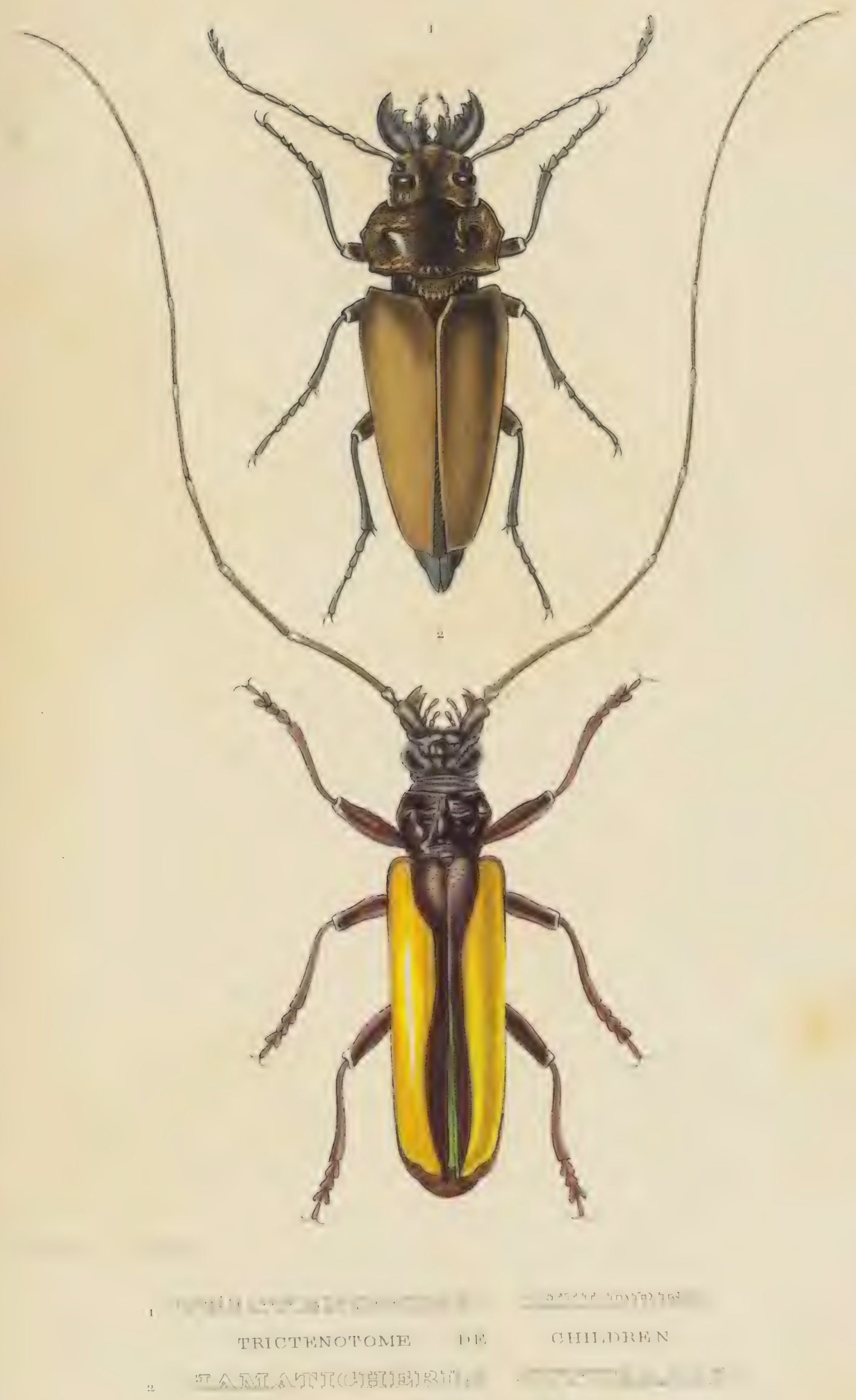

HAMA'ICHERE SITITRAL, 


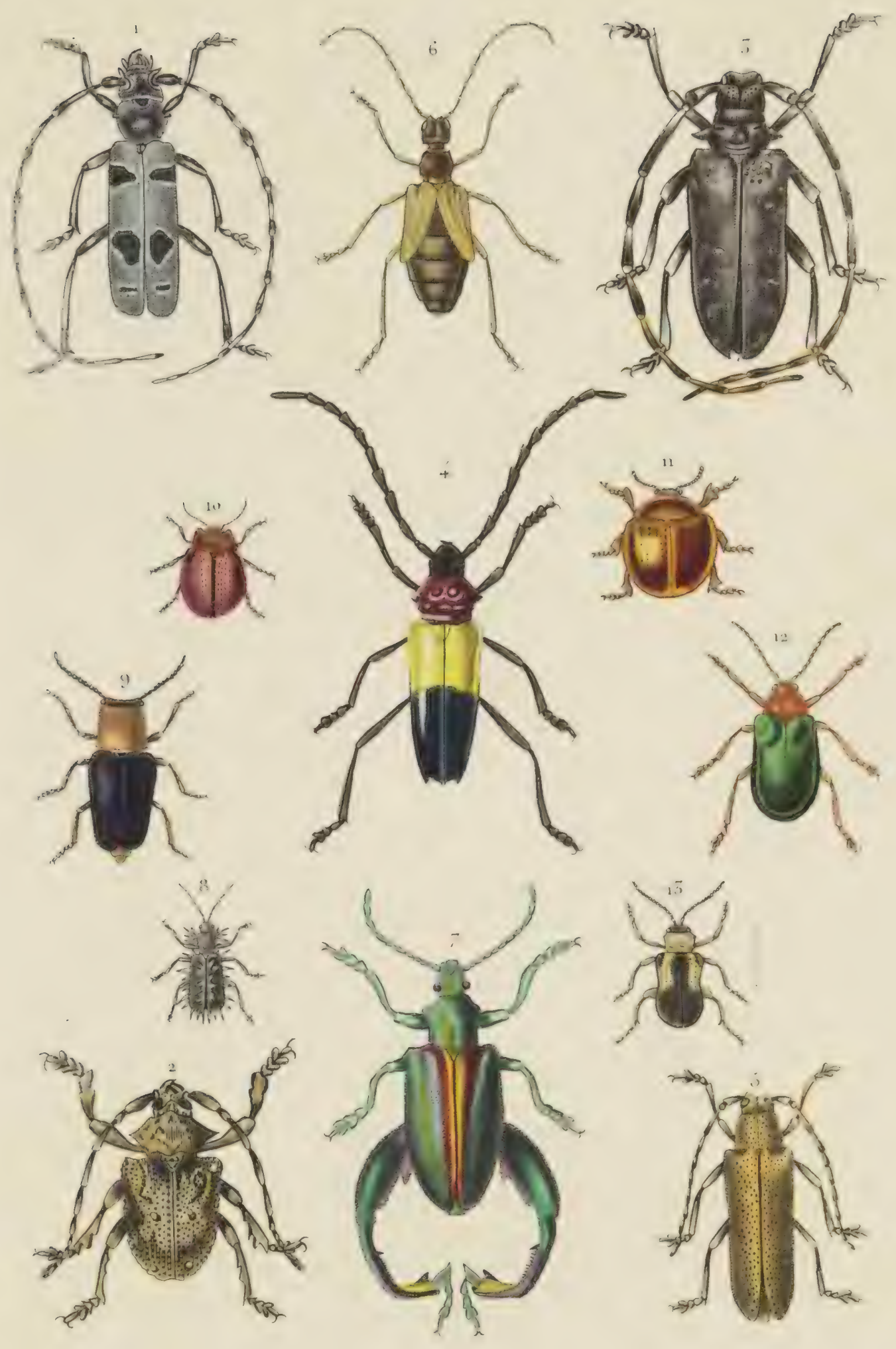

1. ROSALIE des alpes. 5.SAPLRDE carcharias. | 9.MAT ACOGASTRE de passerini 2.0NICHOCERE scorpion. 6.VESPERE gree. 10.CHRYSOAELE Tumerale

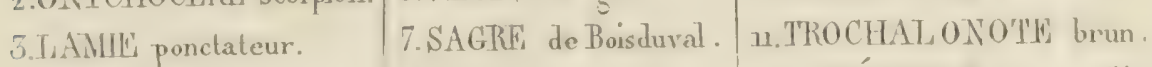
4.P.HCEDLE tricolore. 8.HTSPE defabricius. 12.GALÉRUOUE smaragdine 3.0CTOGONOTH, deBanon. 

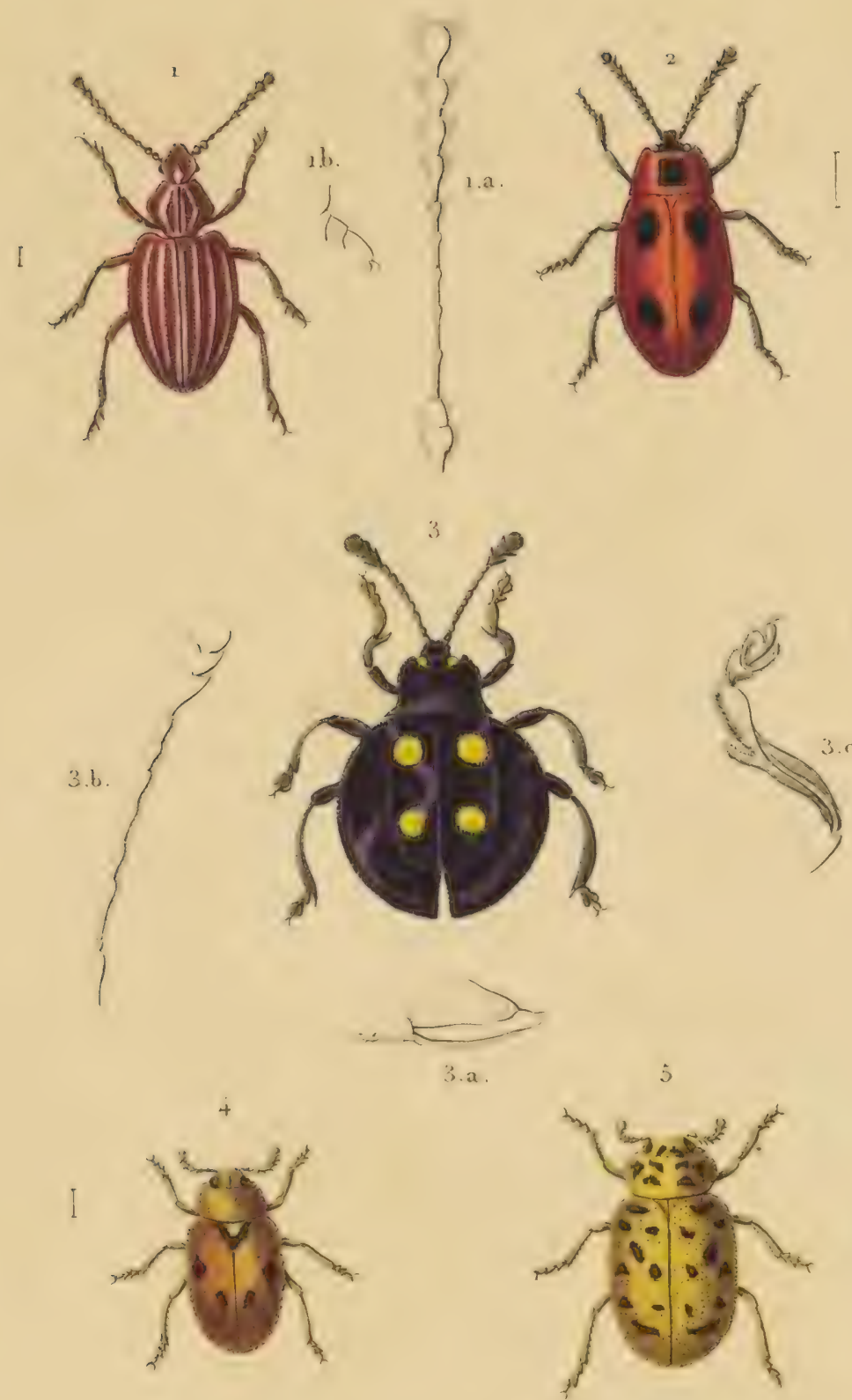
1.DASYCERE sillonó. - 2.ENDOMIQUE écarlate
3.EUMORPHE de Sumatra-4.SCYMNE à écusson
5. COCCLNELLE dix-neuf points. 



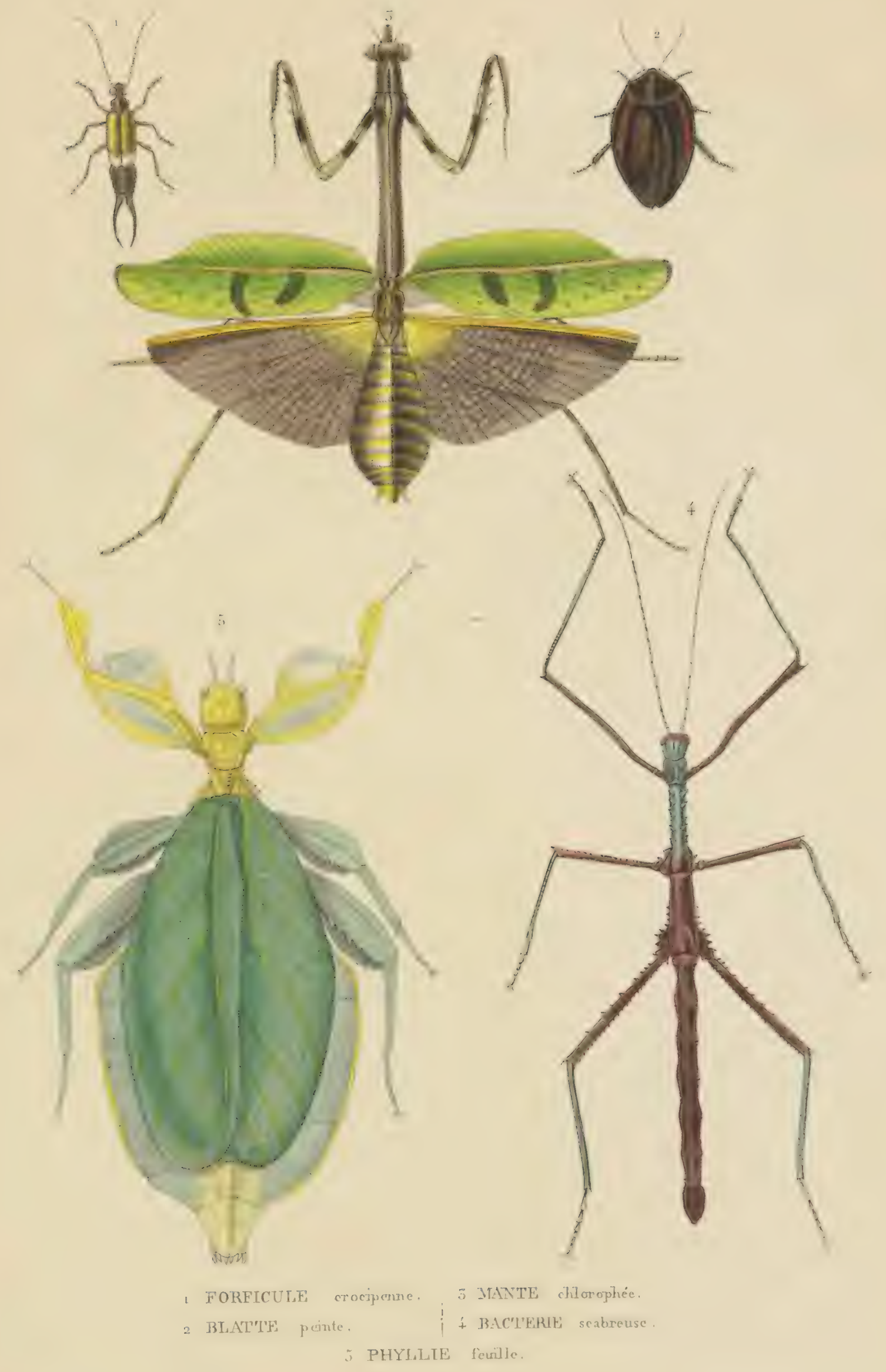



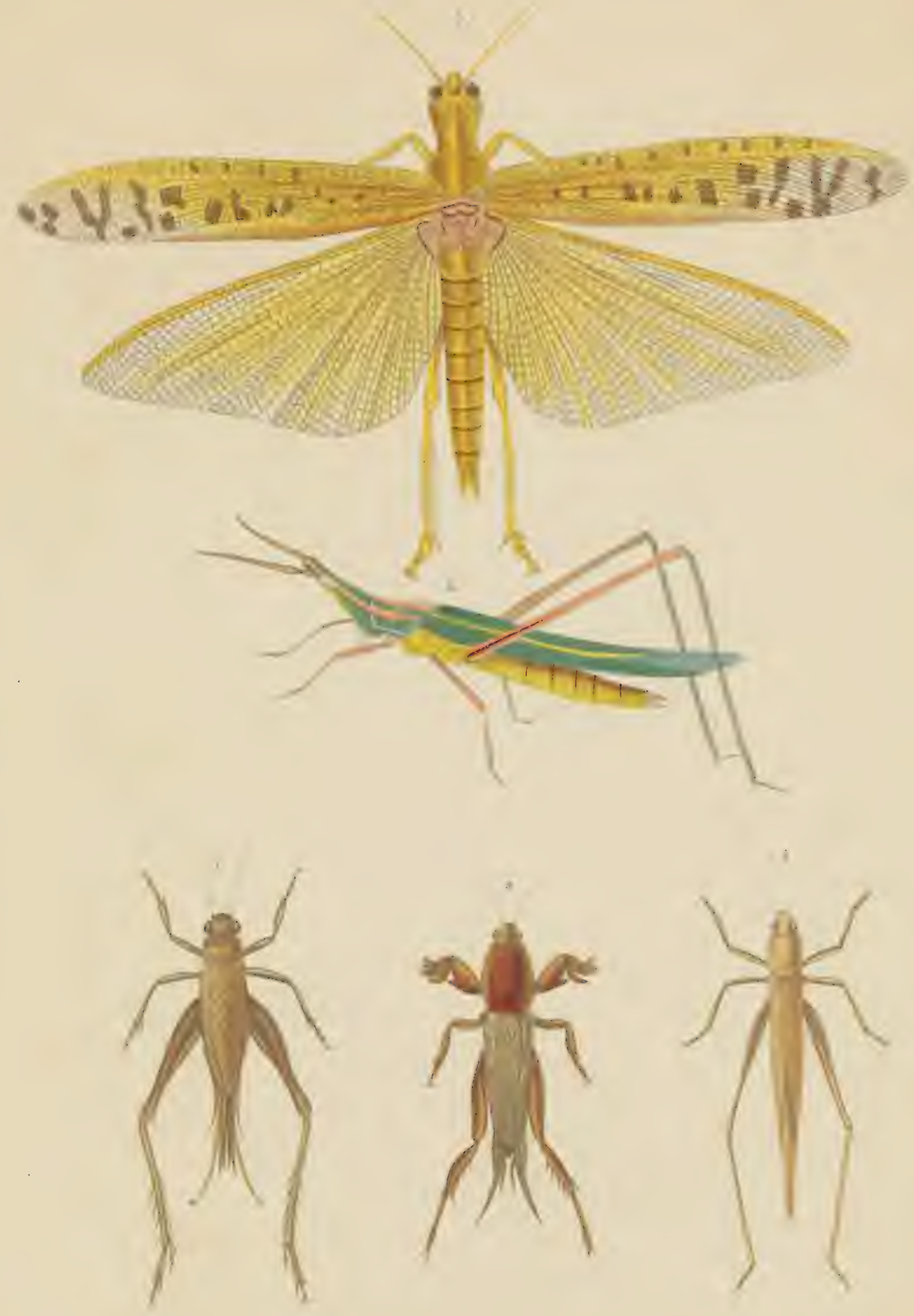

1 GRYLLON Serville

13 SAUTLRFLLi érothrosome

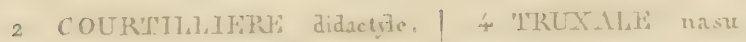

5 C'RLOLE'L' voracenr. 


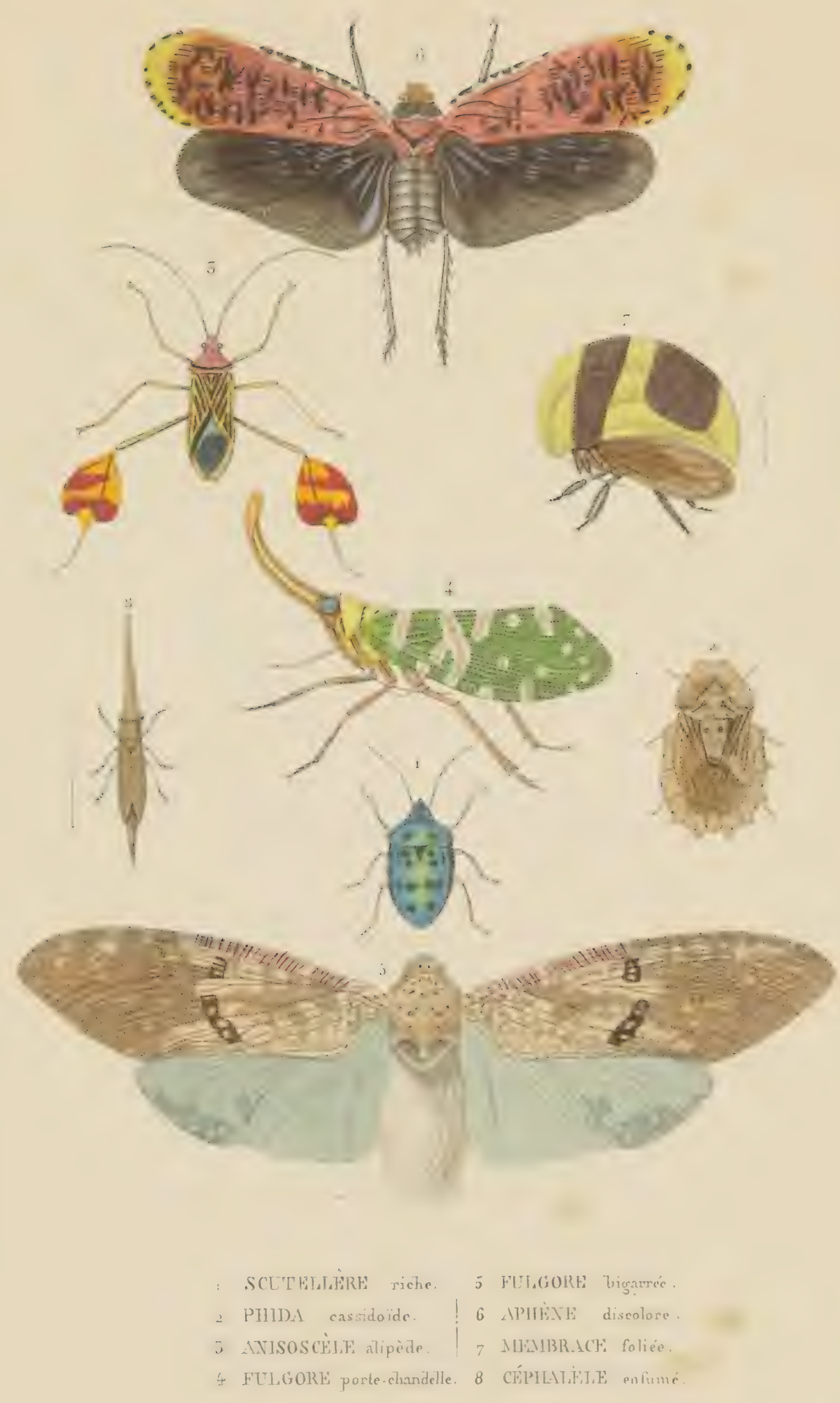




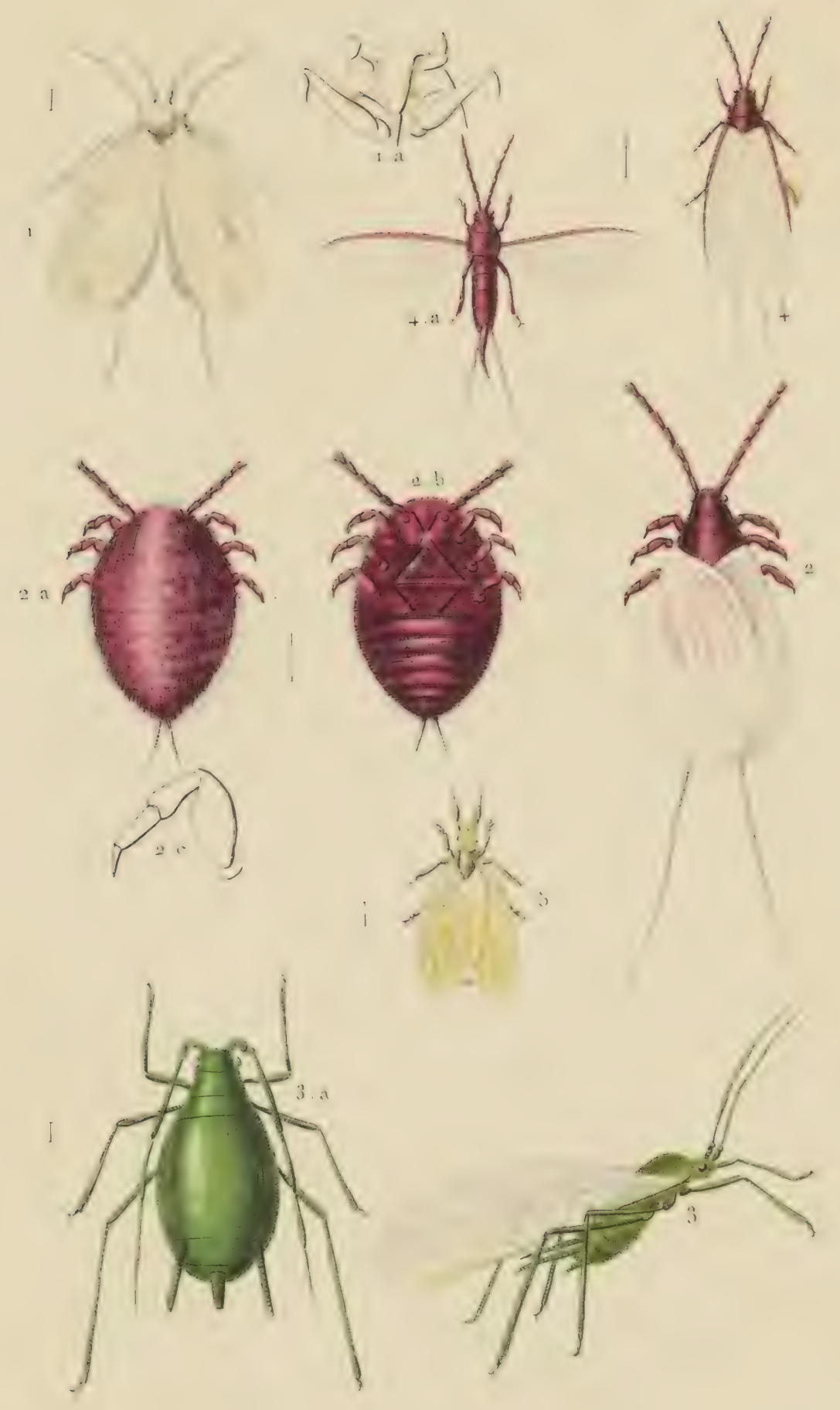

1. ALEYR ODE DE L'ECLATRE. 3.PUCERON DU ROSLER.

2. COCIFNIT, LE DU NOPAL. 4.KFRMES D U PECHER.

5.PSYLTE DE JONC. 


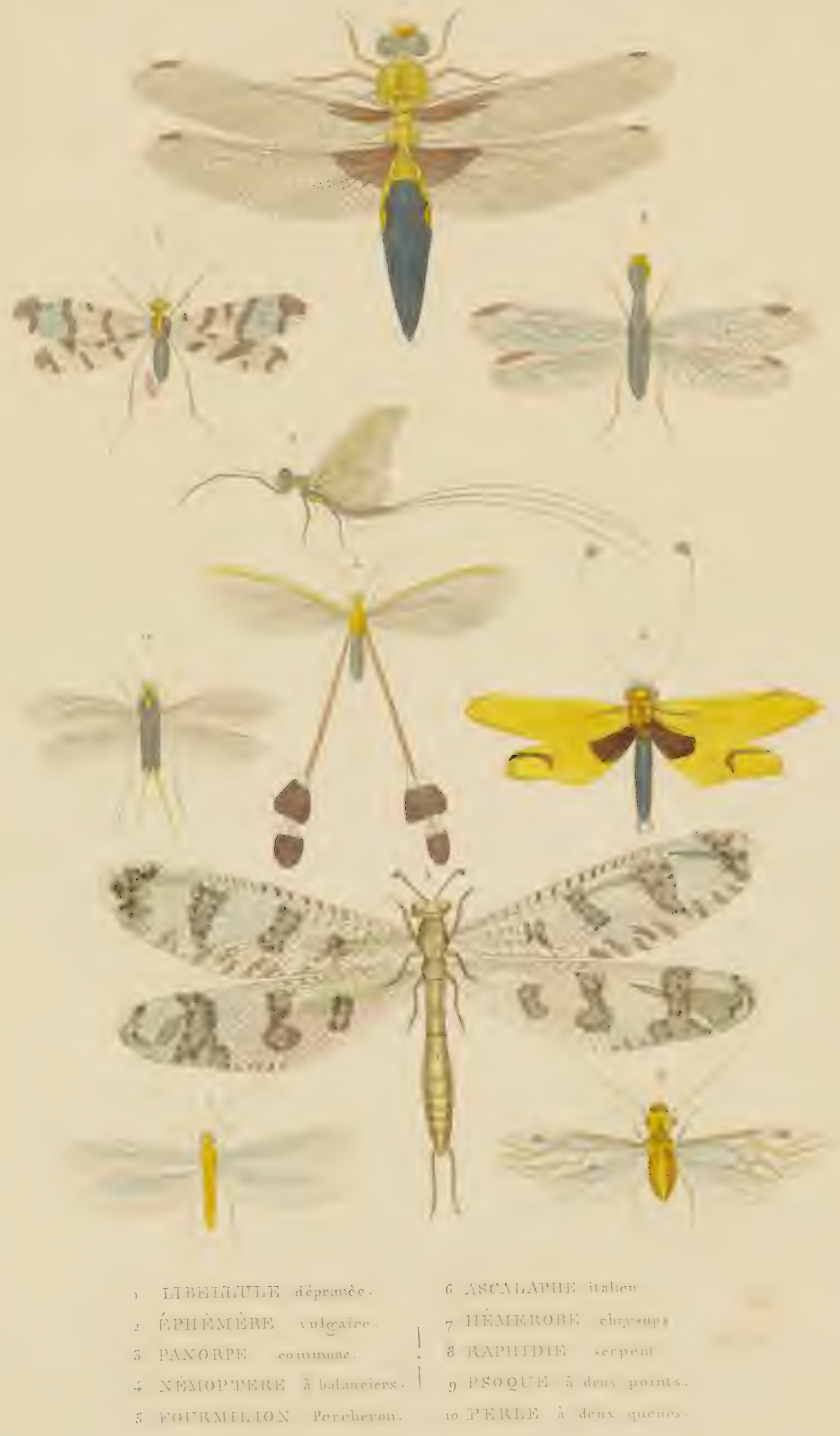



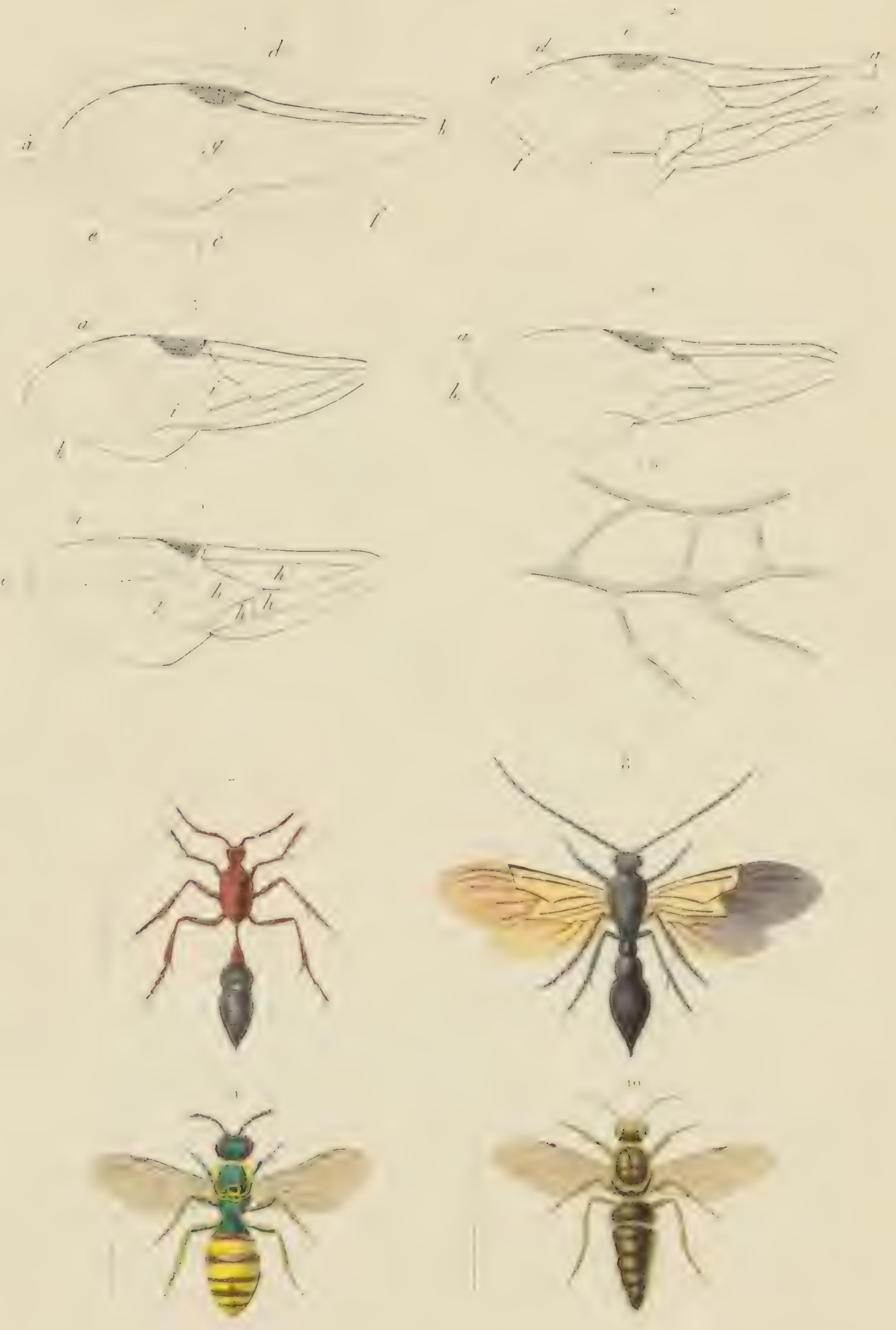

Fig. 1-6. AHLE D'HTMENOPTERBS.

- APTEROGTYE d'alivier... :. AP'T': uniculore

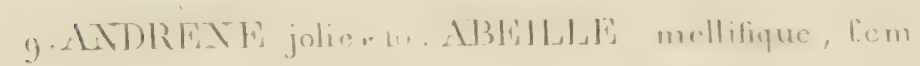




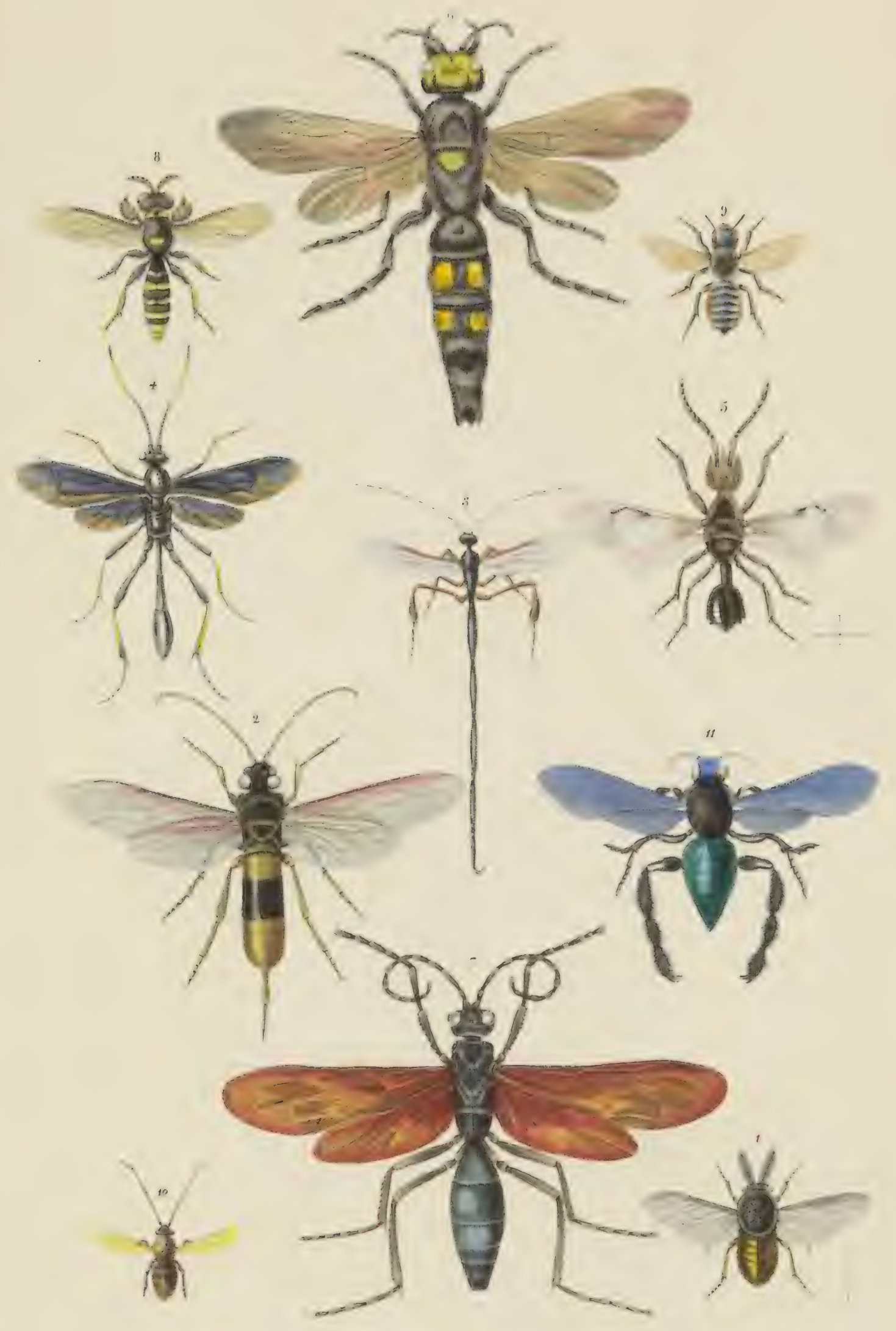
1 HYLO'TOME du rosier.
- SCOIJT des Jardins
" UR O CEHE g'éant.
- PEPSIDE margine
$\therefore$ PELACINE Duponehel.
8 CR\BRON à crible.
+ ospRYNCHO'Tle du cap
(MEGACHILE soyeux
$\therefore$ CéROCLPHAL cornigere.
10 MELISSODE de fonscolombe

"A CANTHOPE splendide. 

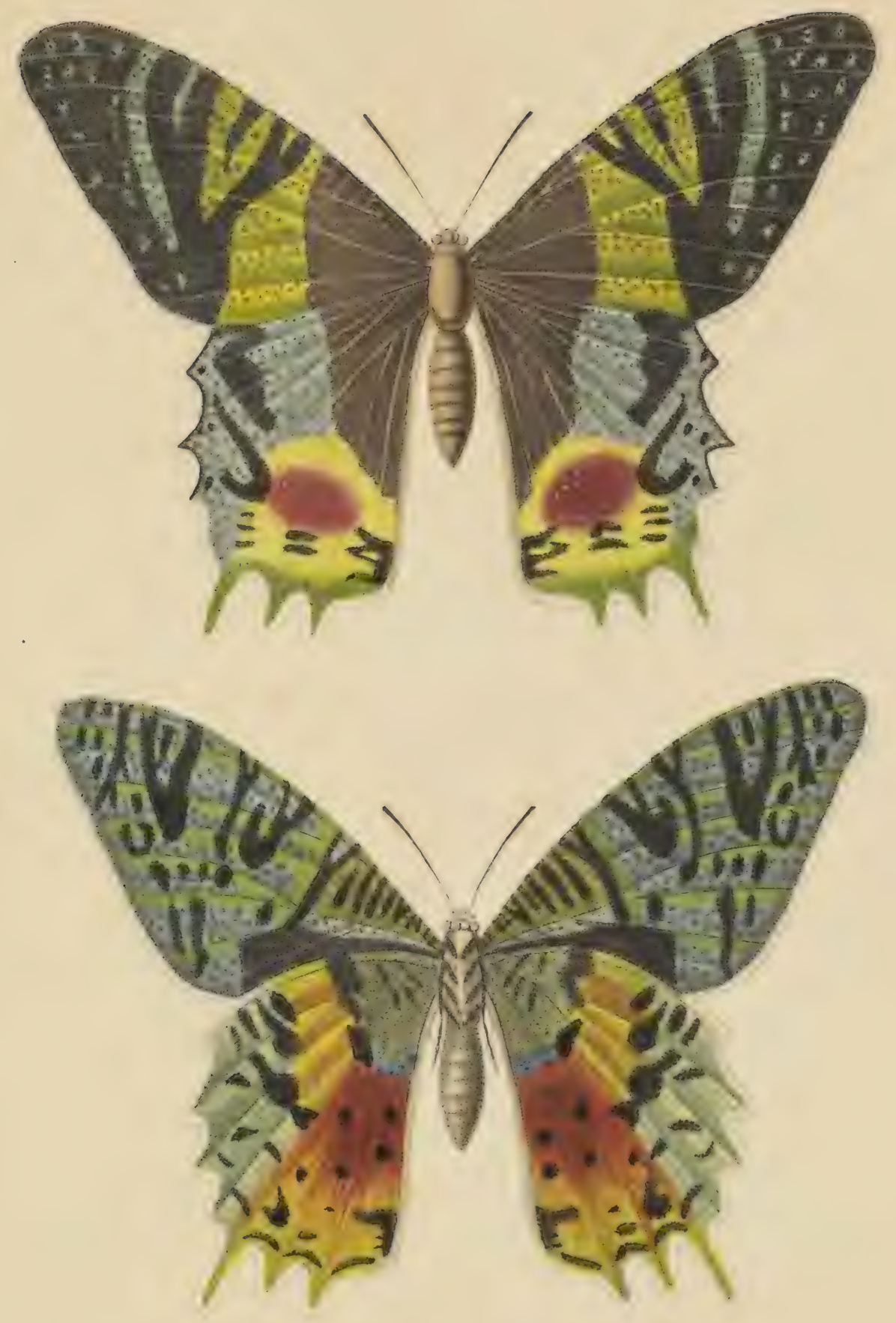

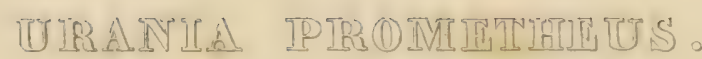

URANTE PROIETHEE. 

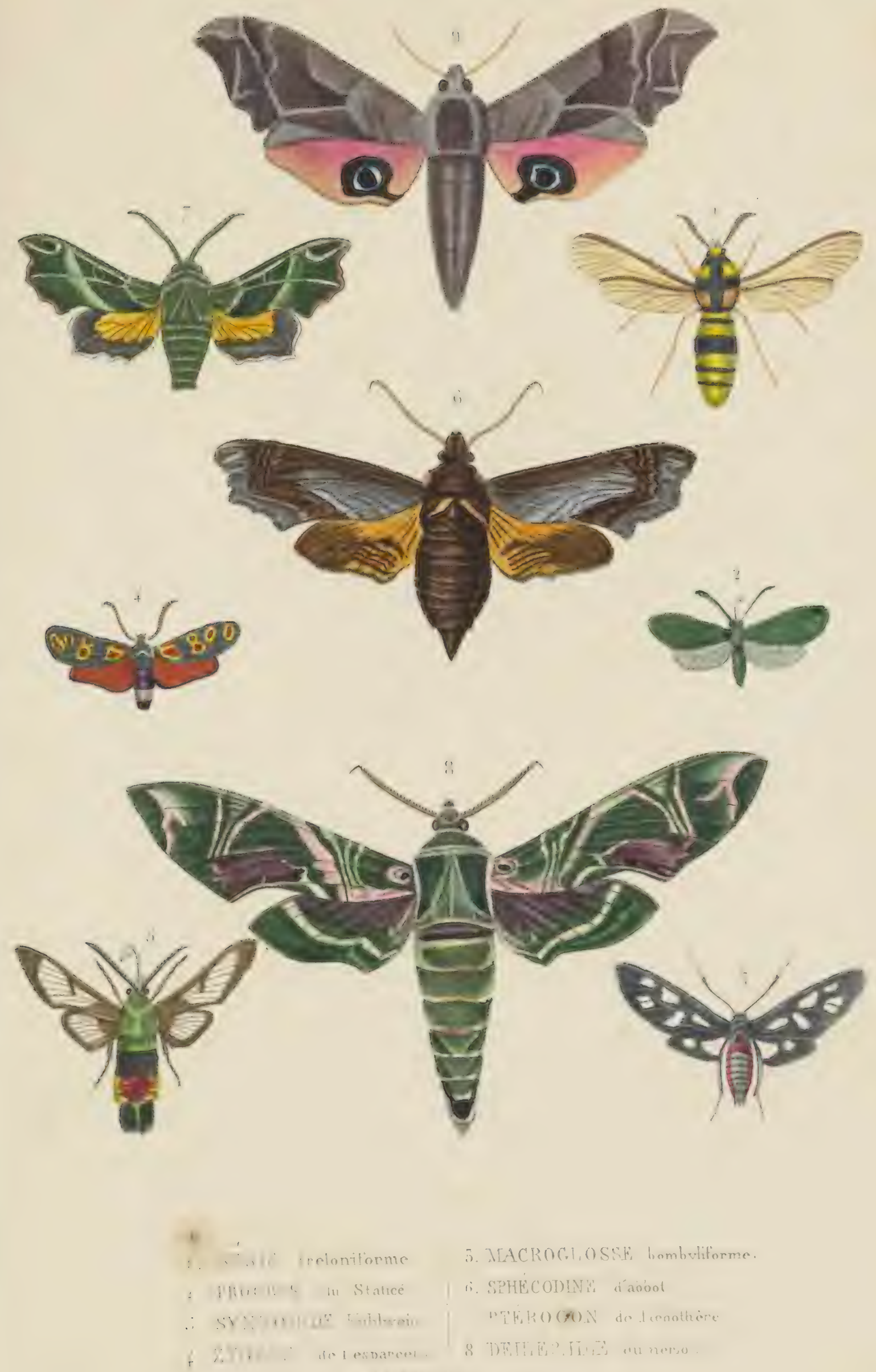

$40 \log 1$, oretent 

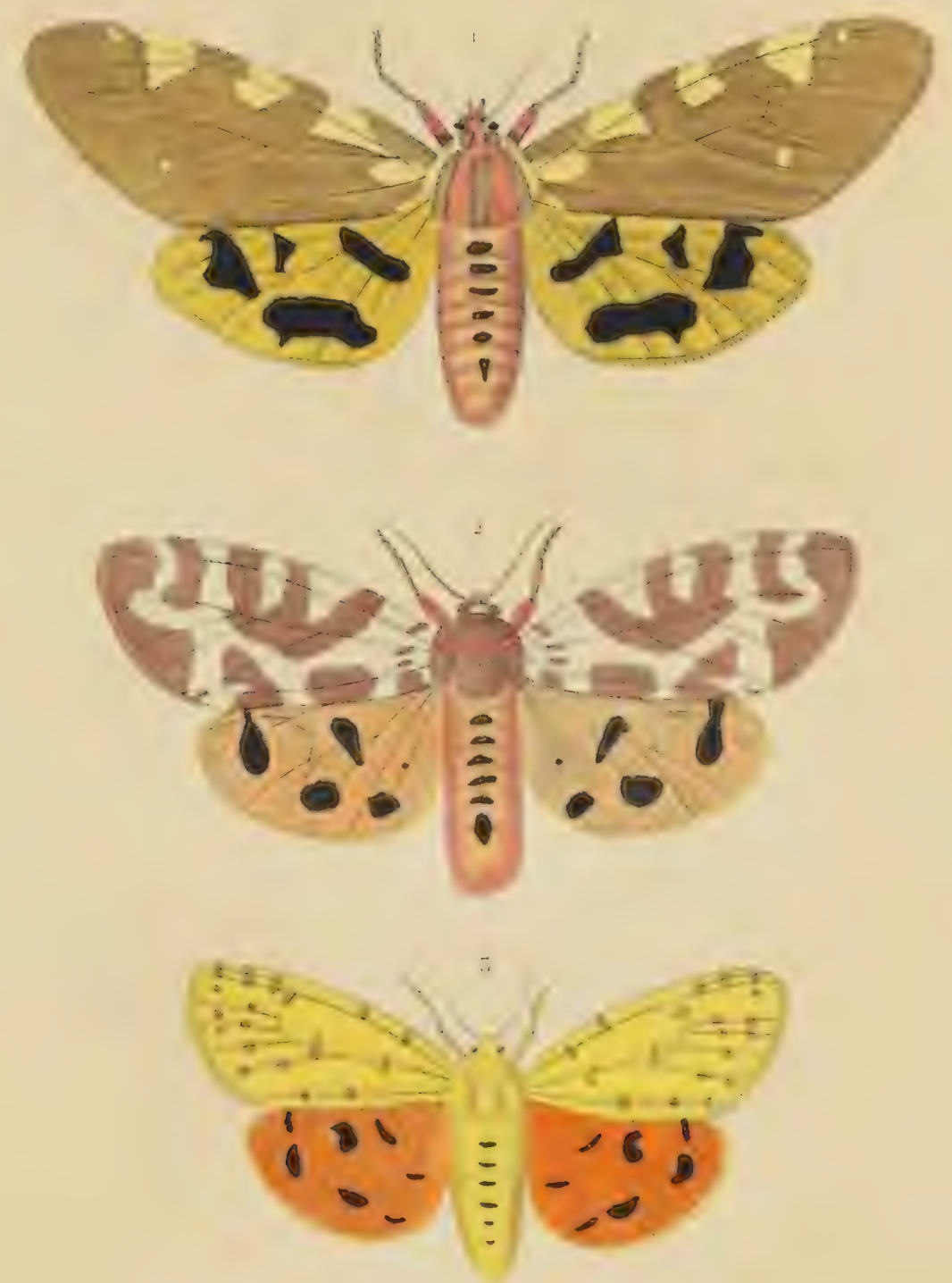
1. ARCTCJA matronula -BOMBICF matrome
2. $\triangle 0$ C Casa. - I . Bérisson
5. $\Delta$ o o purpurea. B moucheté . 


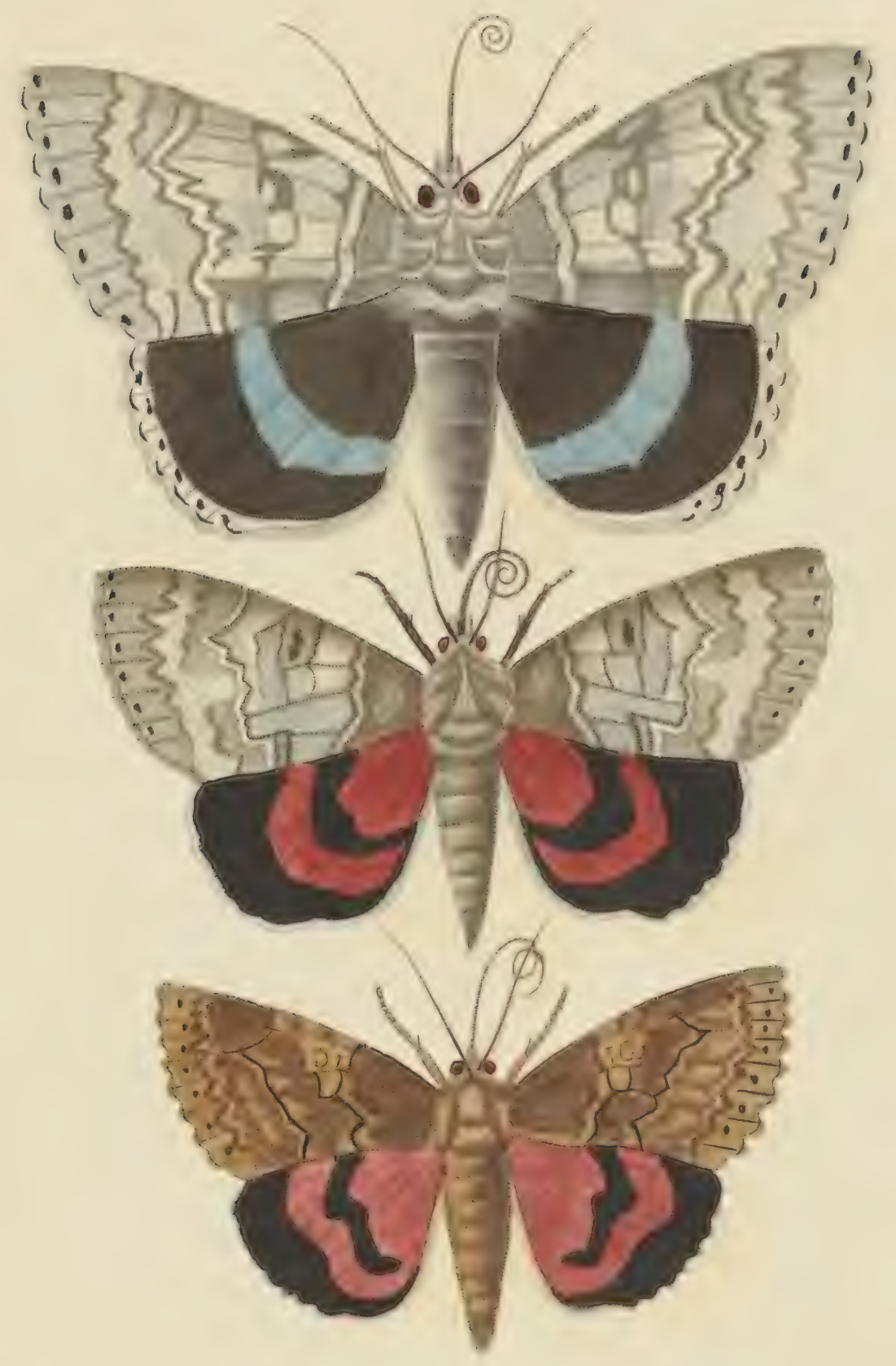

- NOCTETA IETRA.TITNIL O

NOCTUELEA DI FREN

2.NOCIEUA STP (1) NNSA -

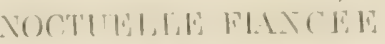

3NOCGIOTA NVUTPILA。

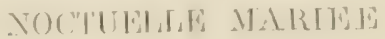




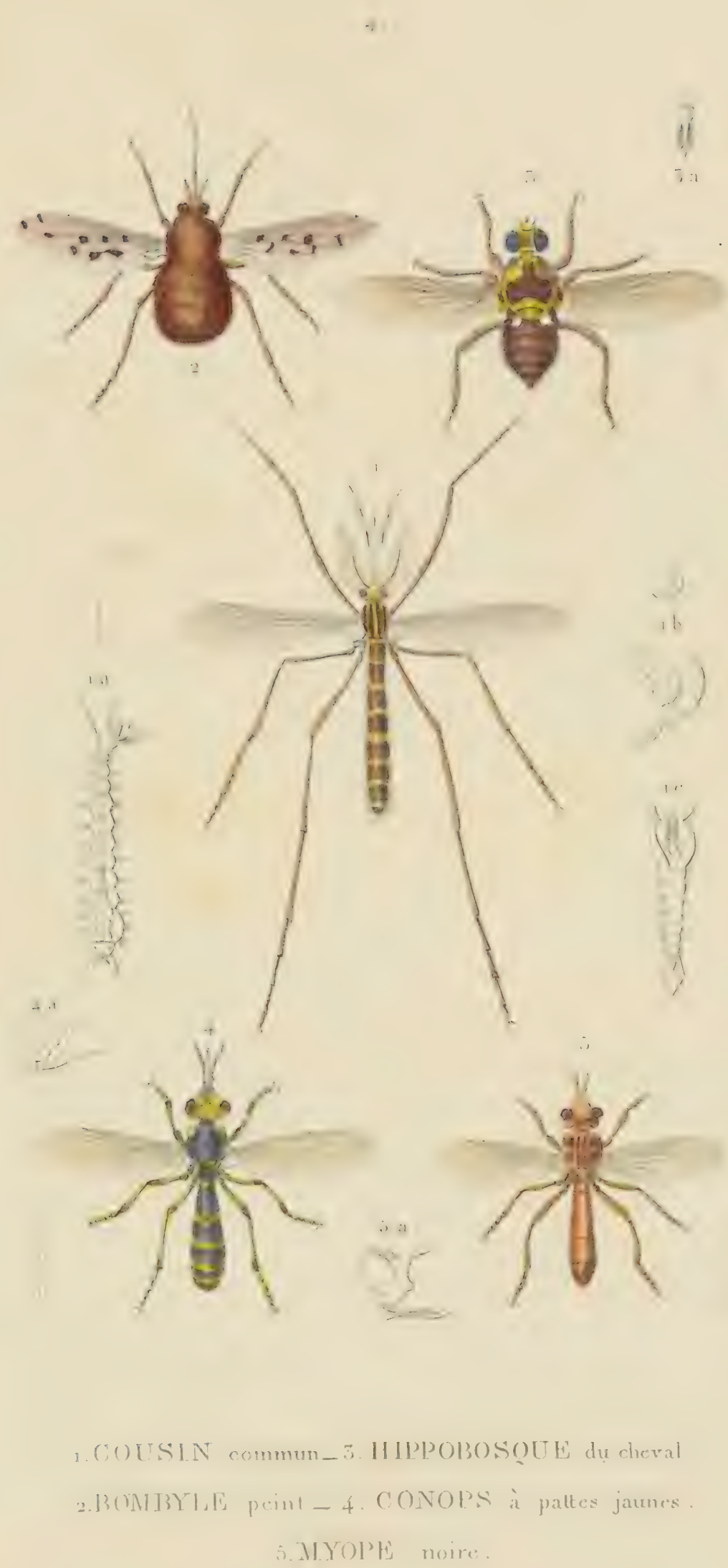




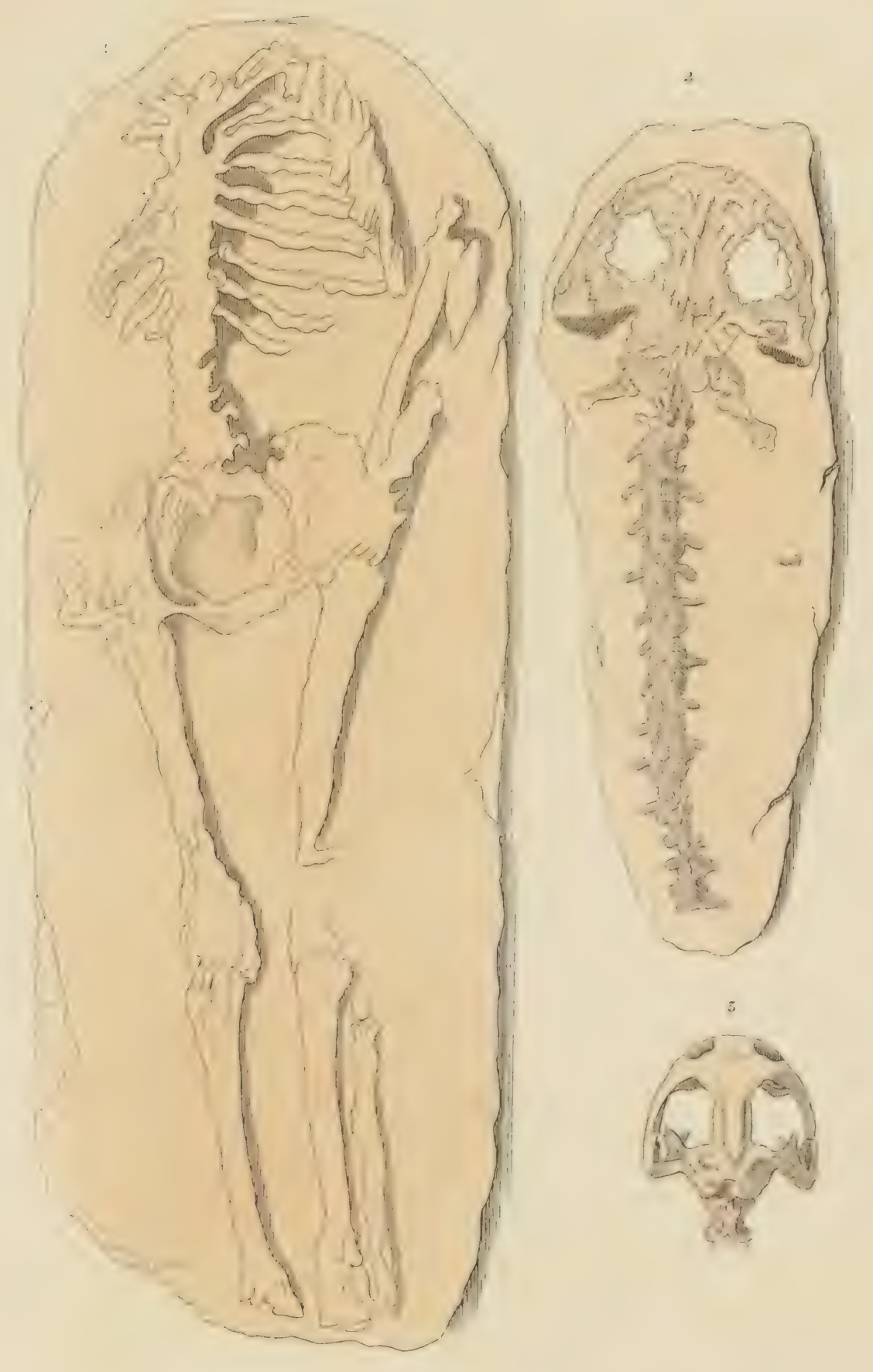

1. ANTIBIROIPOILITIE

2. IPIROIOIEIE。

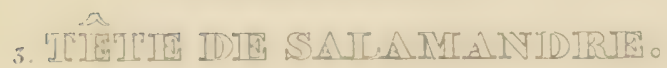




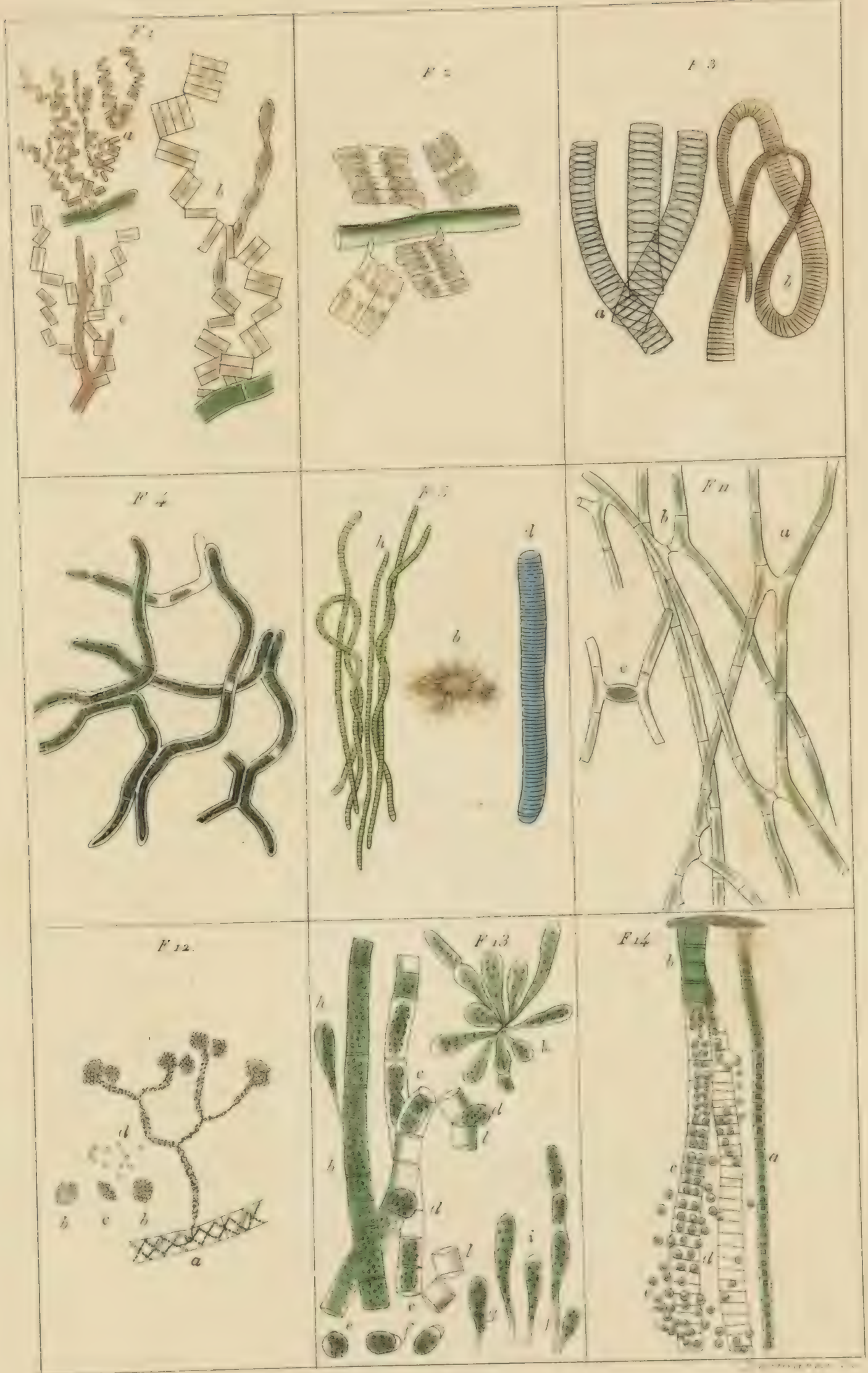

A. 1. . 


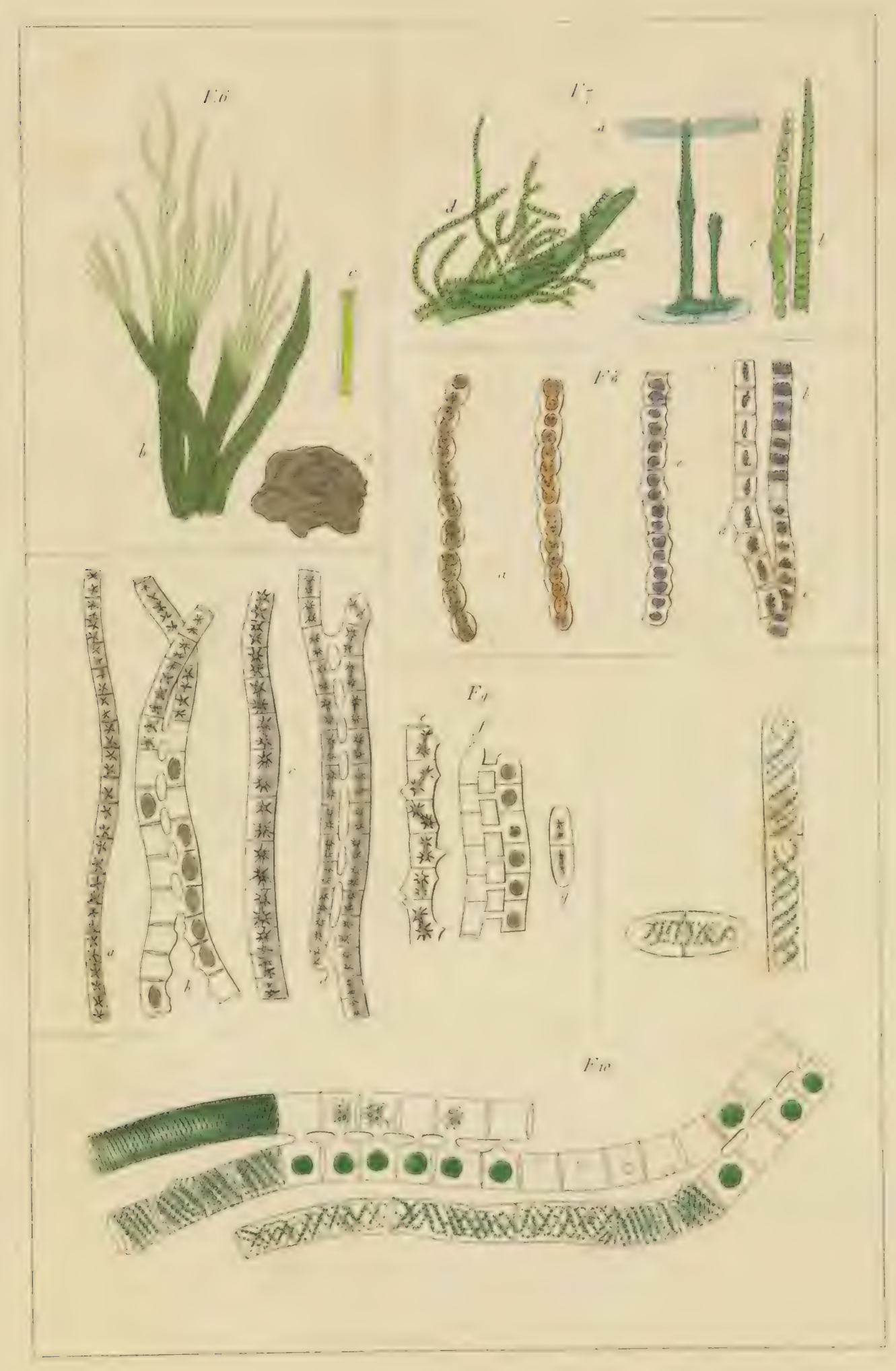



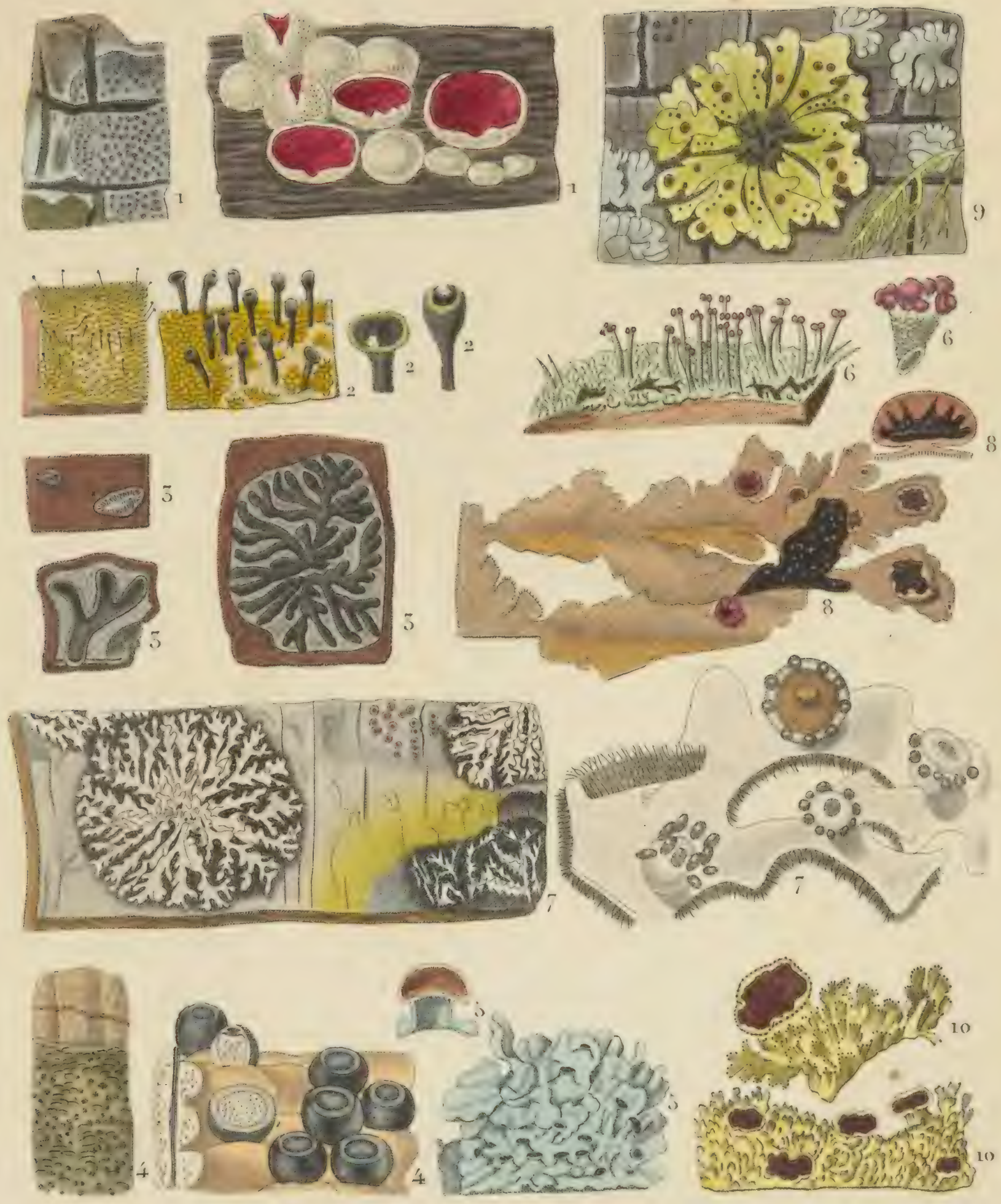

\section{I.ICHE. N S .}

1. GASSICOURTIE Ecarlate.

2. CALYCIER Chrysocéphale

5. SARCOGRAPHE Des quinquimas

4. VERRUCAliE 1)e gaudichaud.

$\therefore$ COLLFIIA Aruté.
6. SCYPHOT'HORE Didyme .

־ PARMELIE Couromać.

8. PIRC'TOCARLON Je delise.

9. SOLORINE Des quinguinas .

ı. CETRAIRE Du gुeniévrier. 

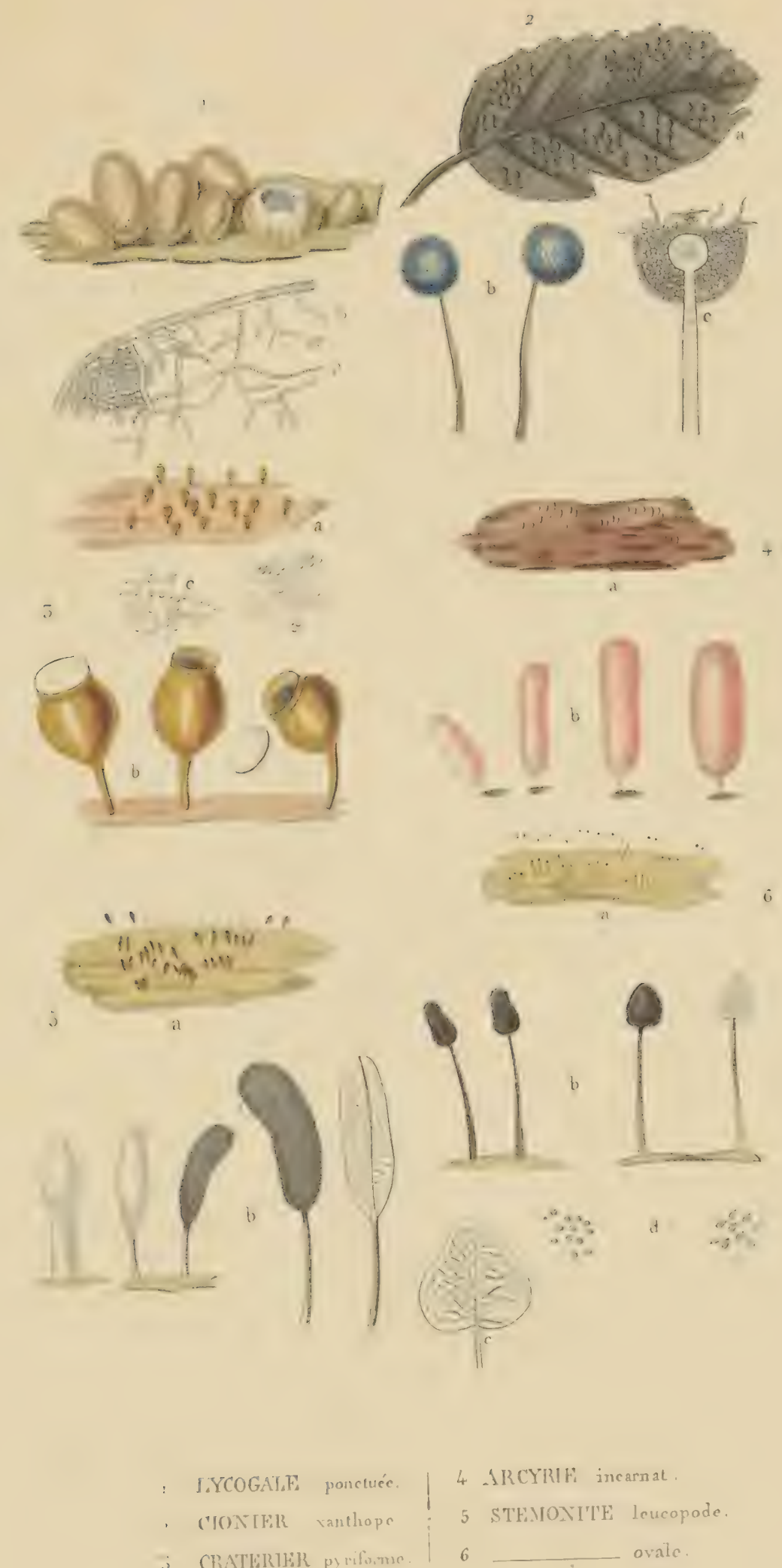

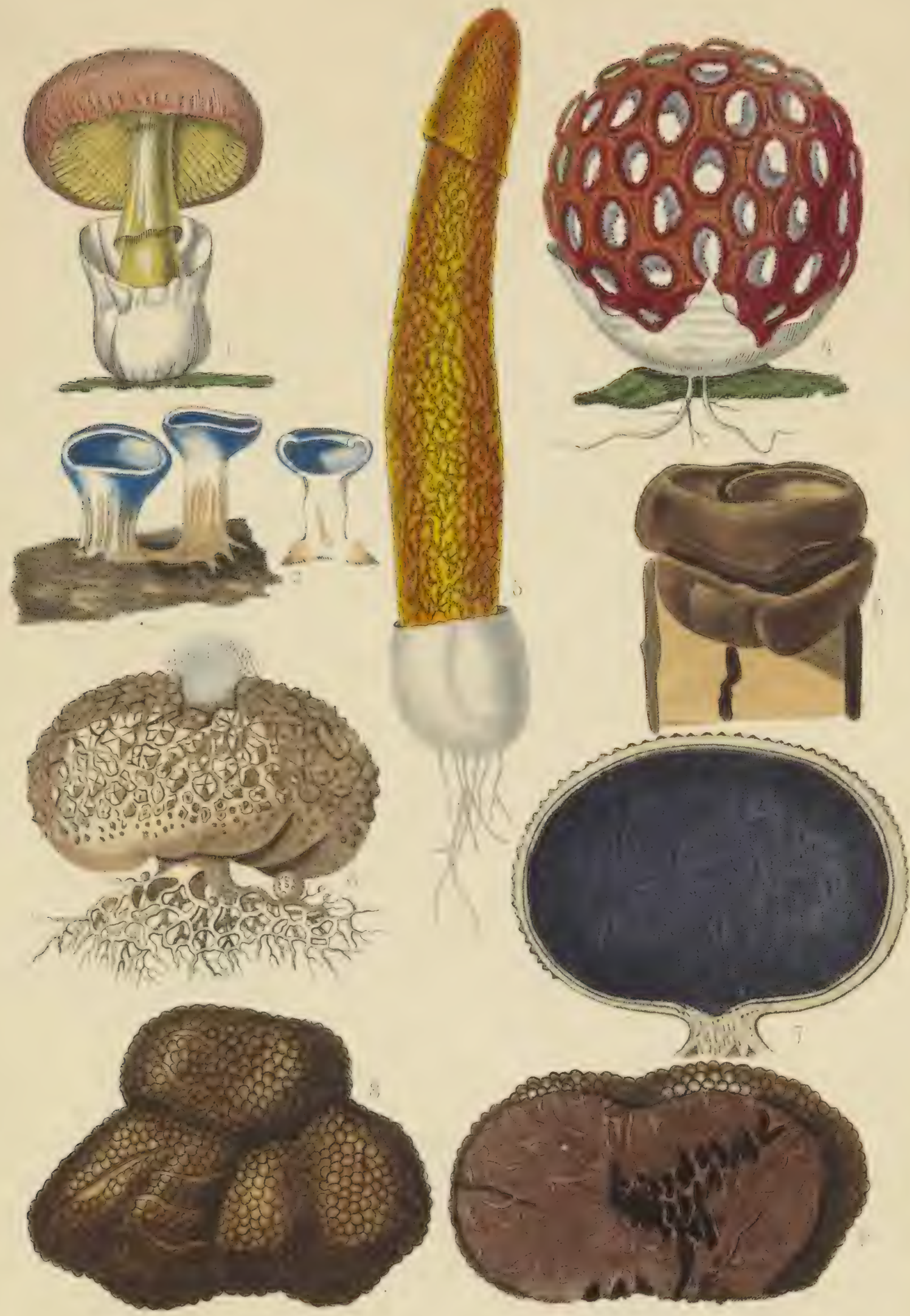

ICARTC, Oronce

2. PEZIZE Bleu

- 'PREMEILE Oreille de Judas

4. CLATHRE Fri.

PHALLUS Oranóc

6. LYCOPLRDON Cisel

IYCOPERDON Gionantesque coupe. 8.9. TRUFIE 


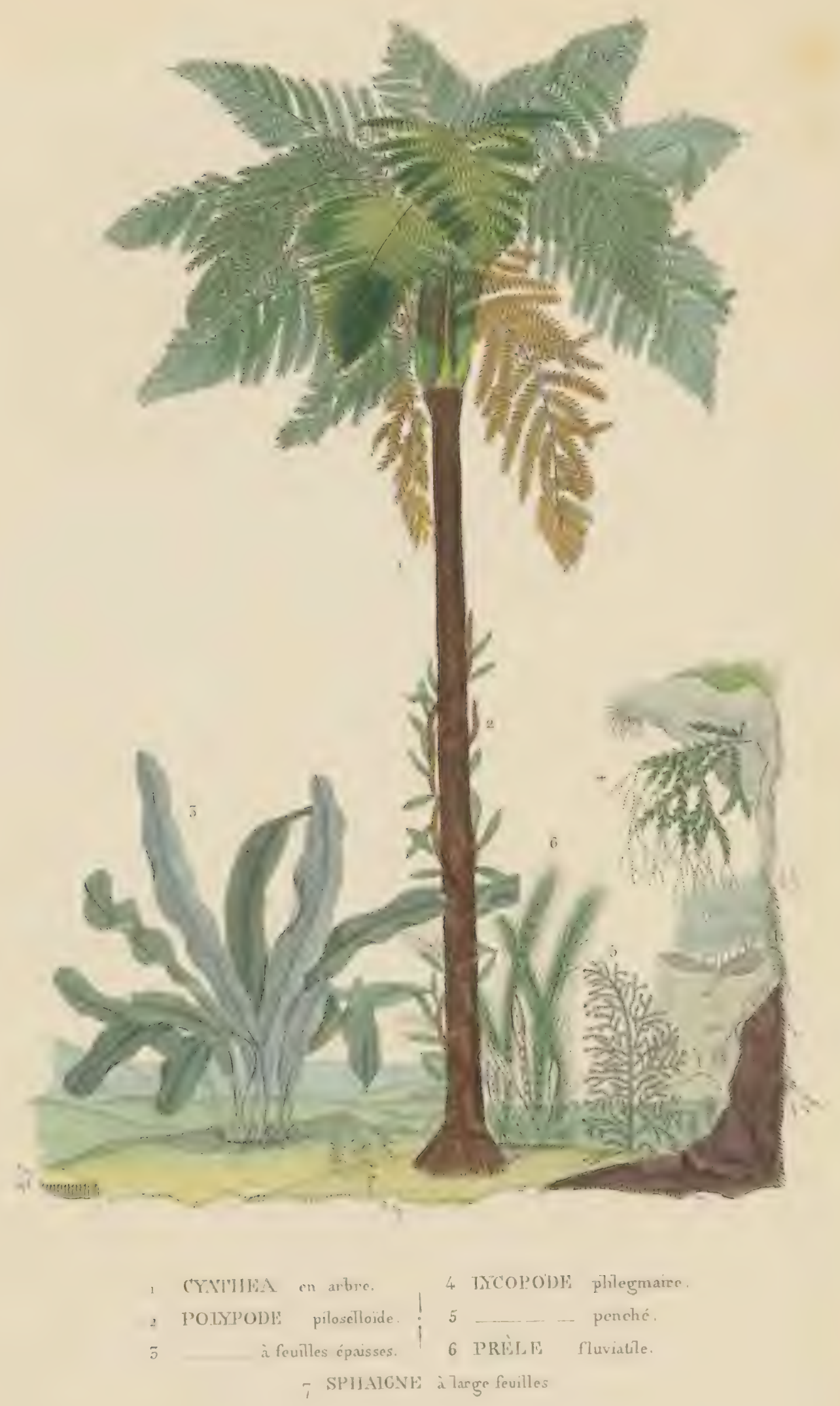




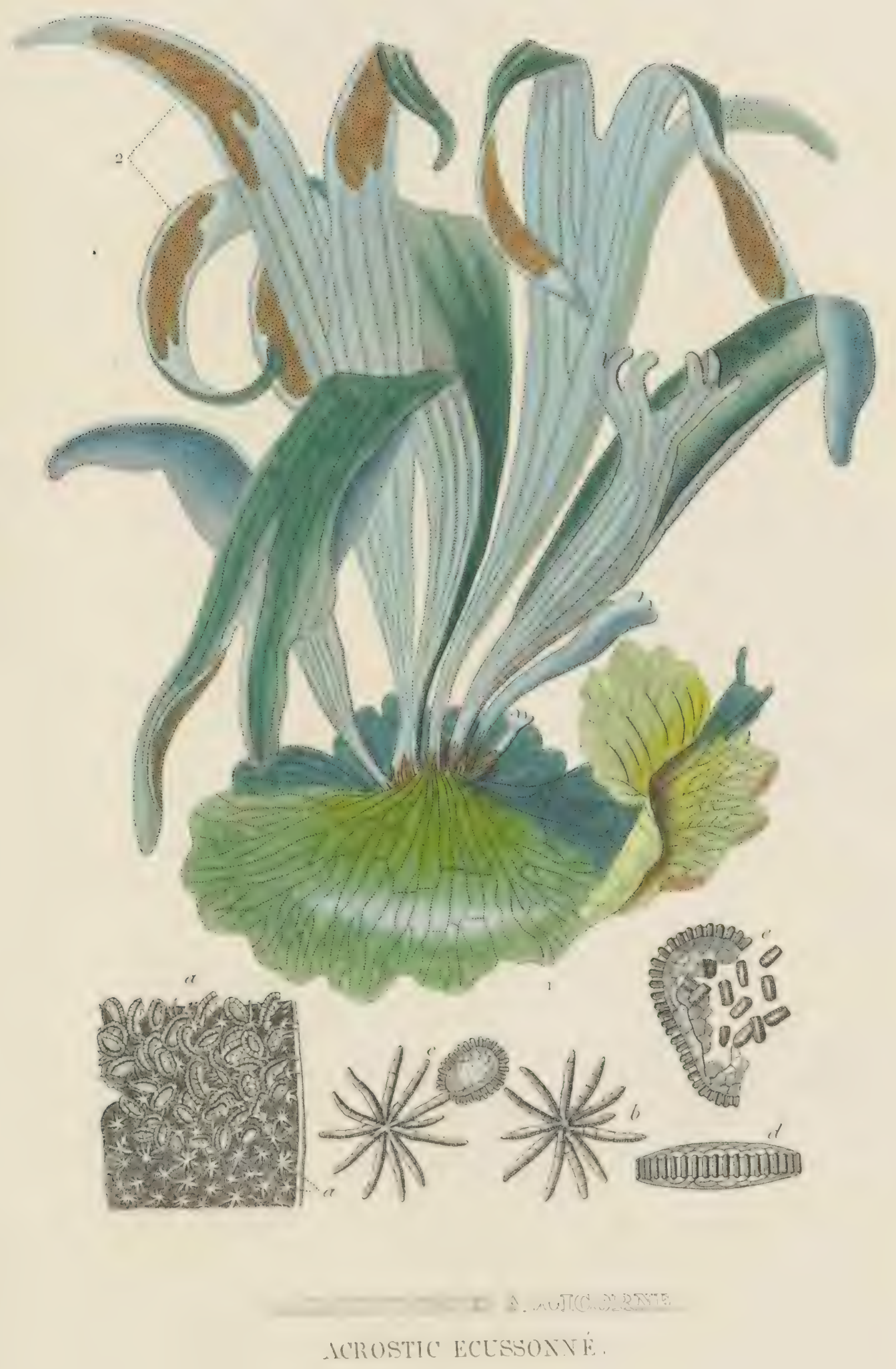




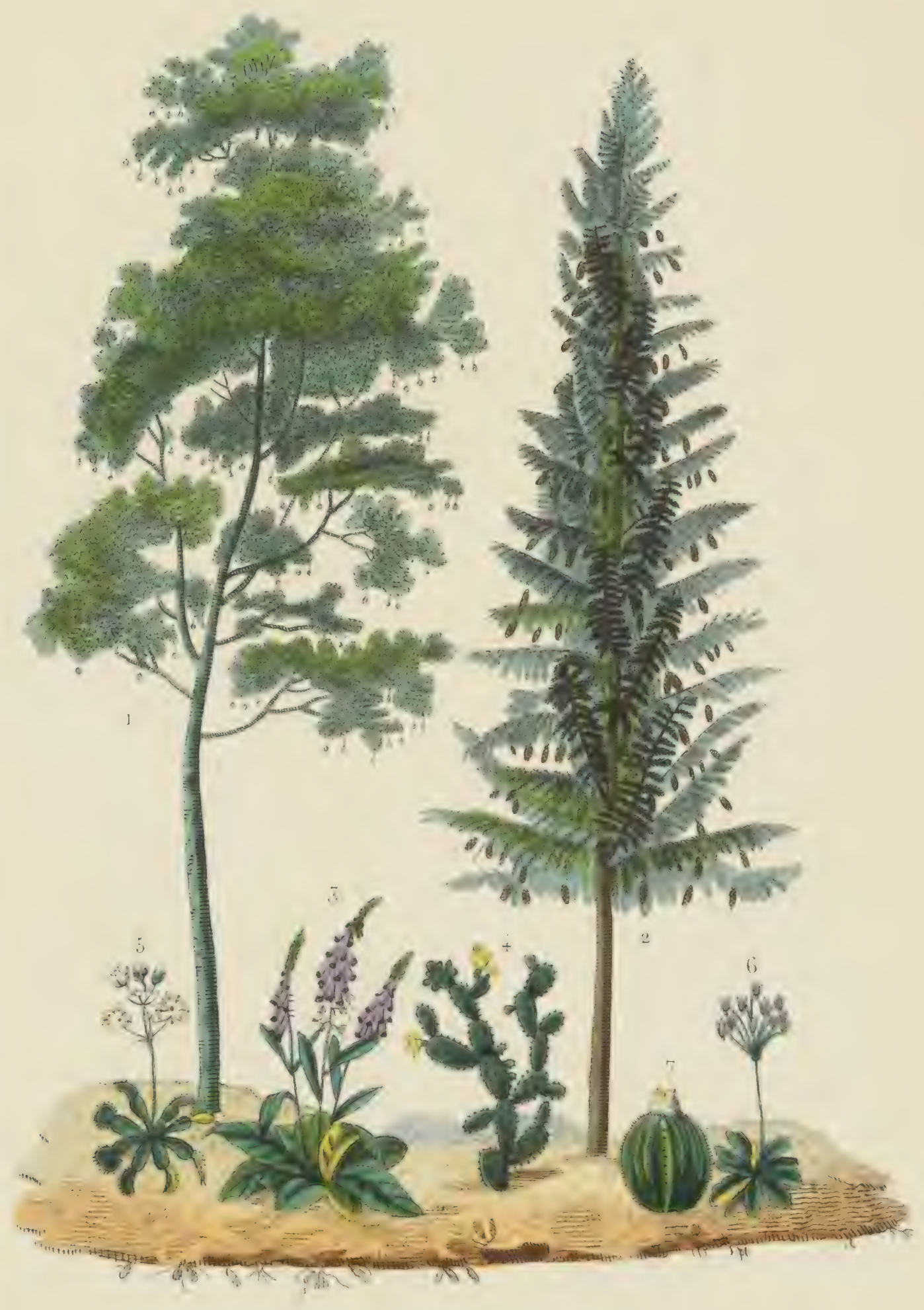

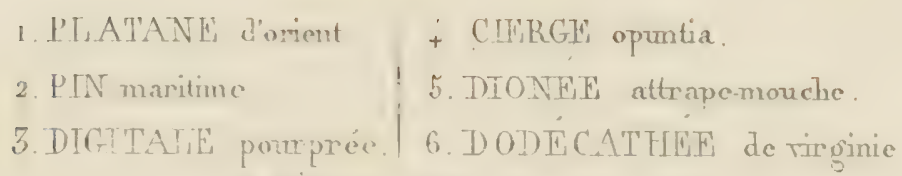

MVLCCACTE déprimé 


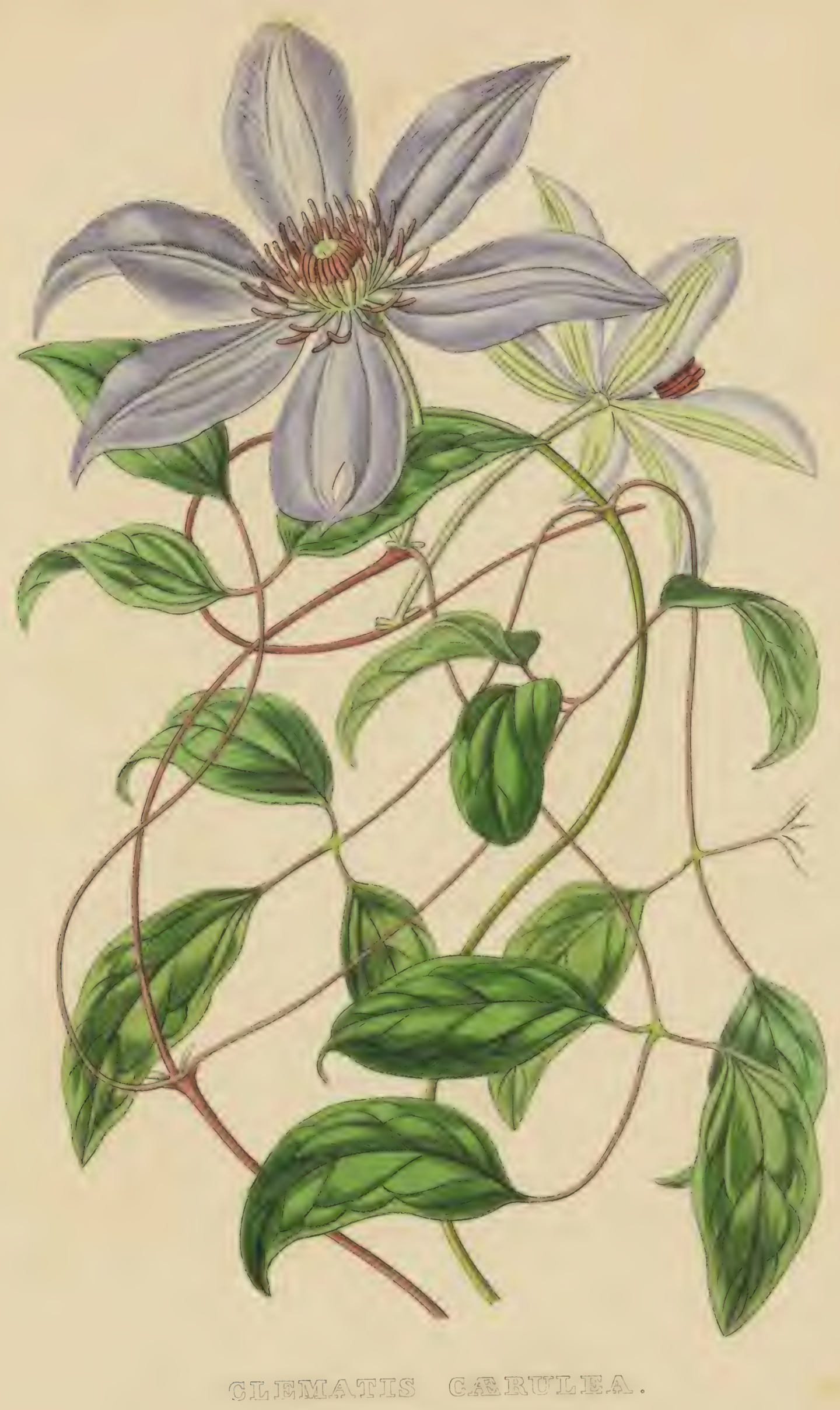

CJFimATITE VIOLFTTF.

Desinares se 


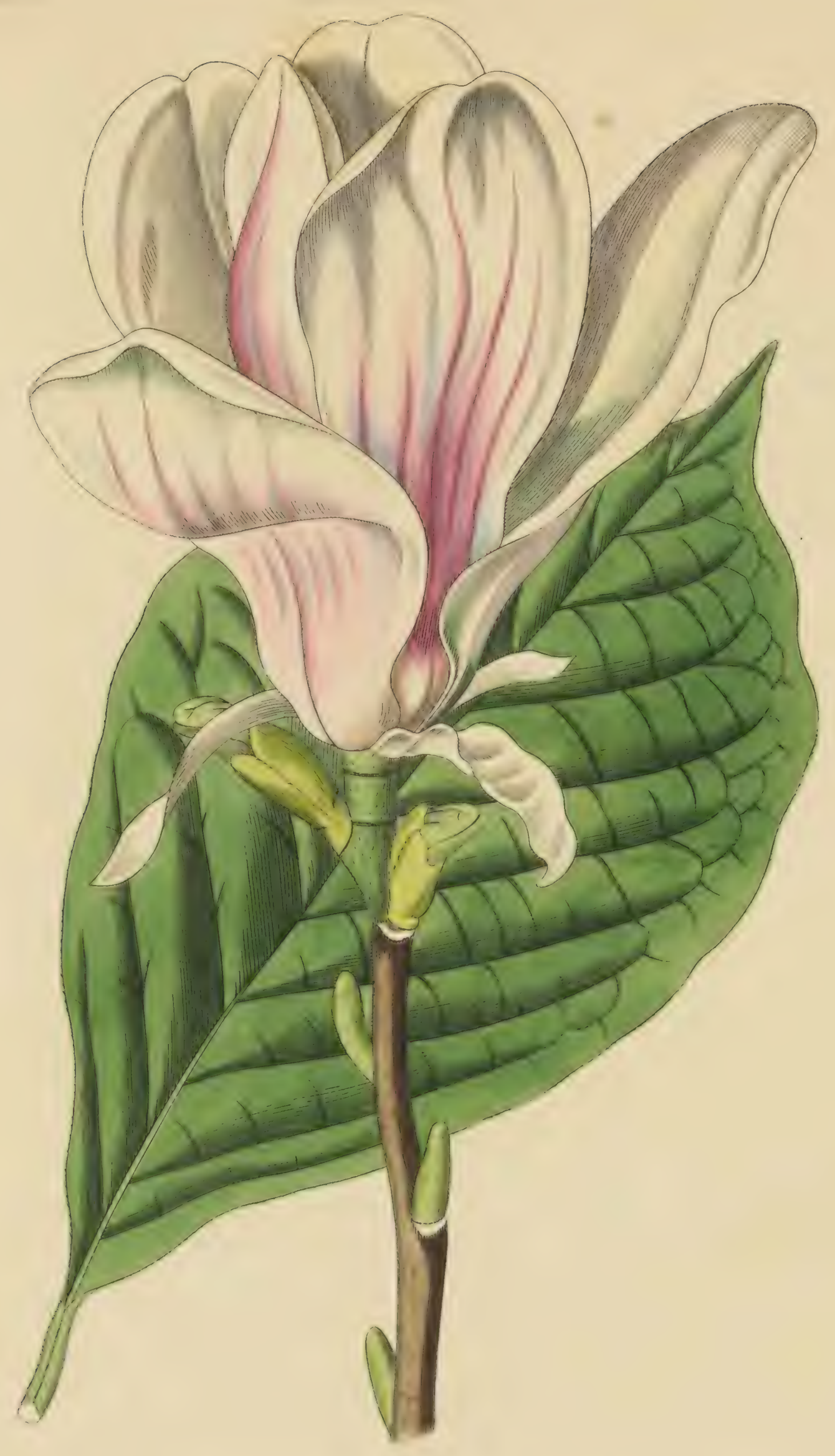

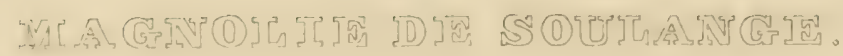

MAGNOIIA SOULANGIANA. 


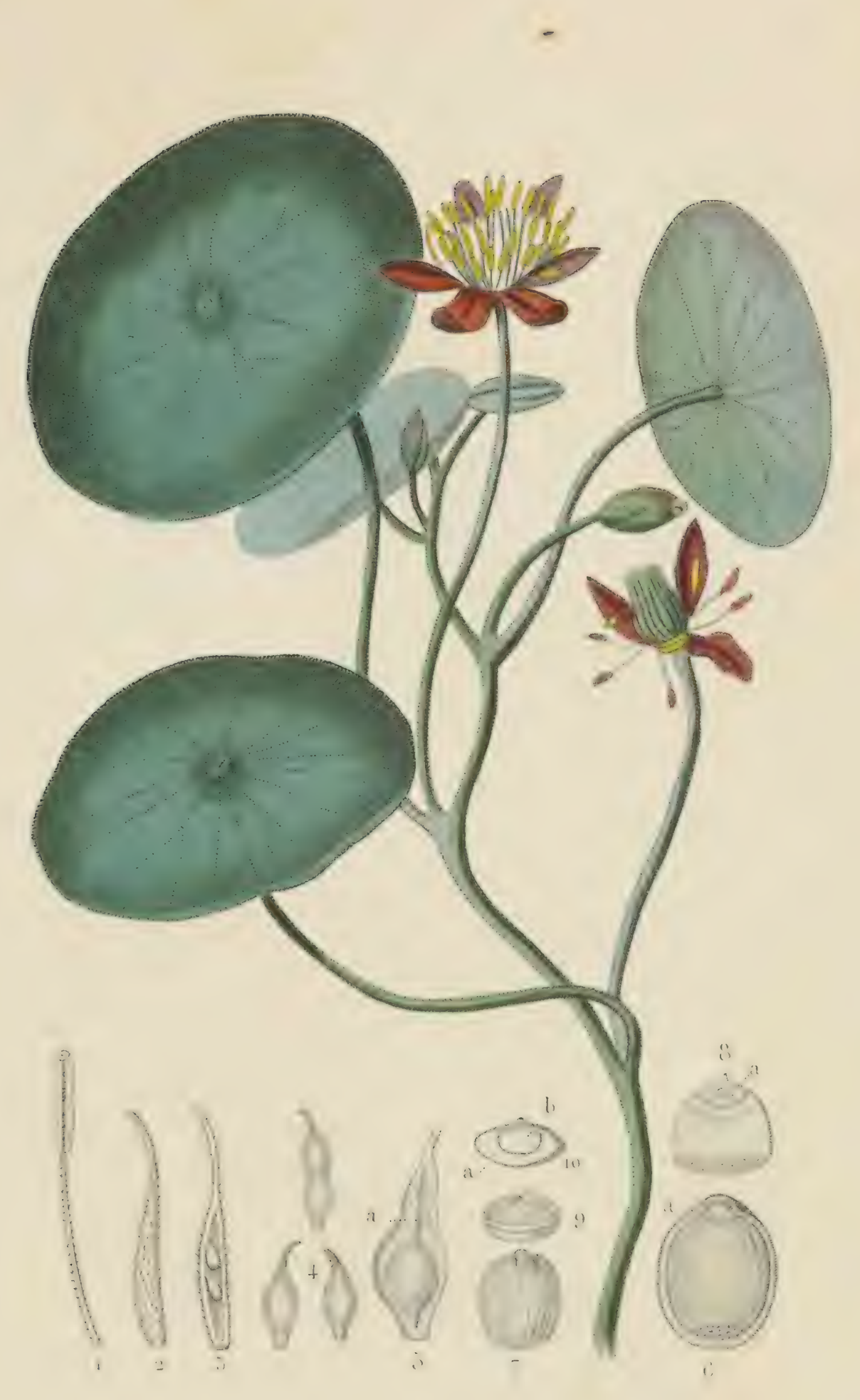

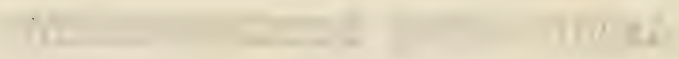

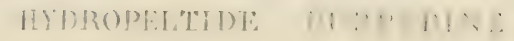




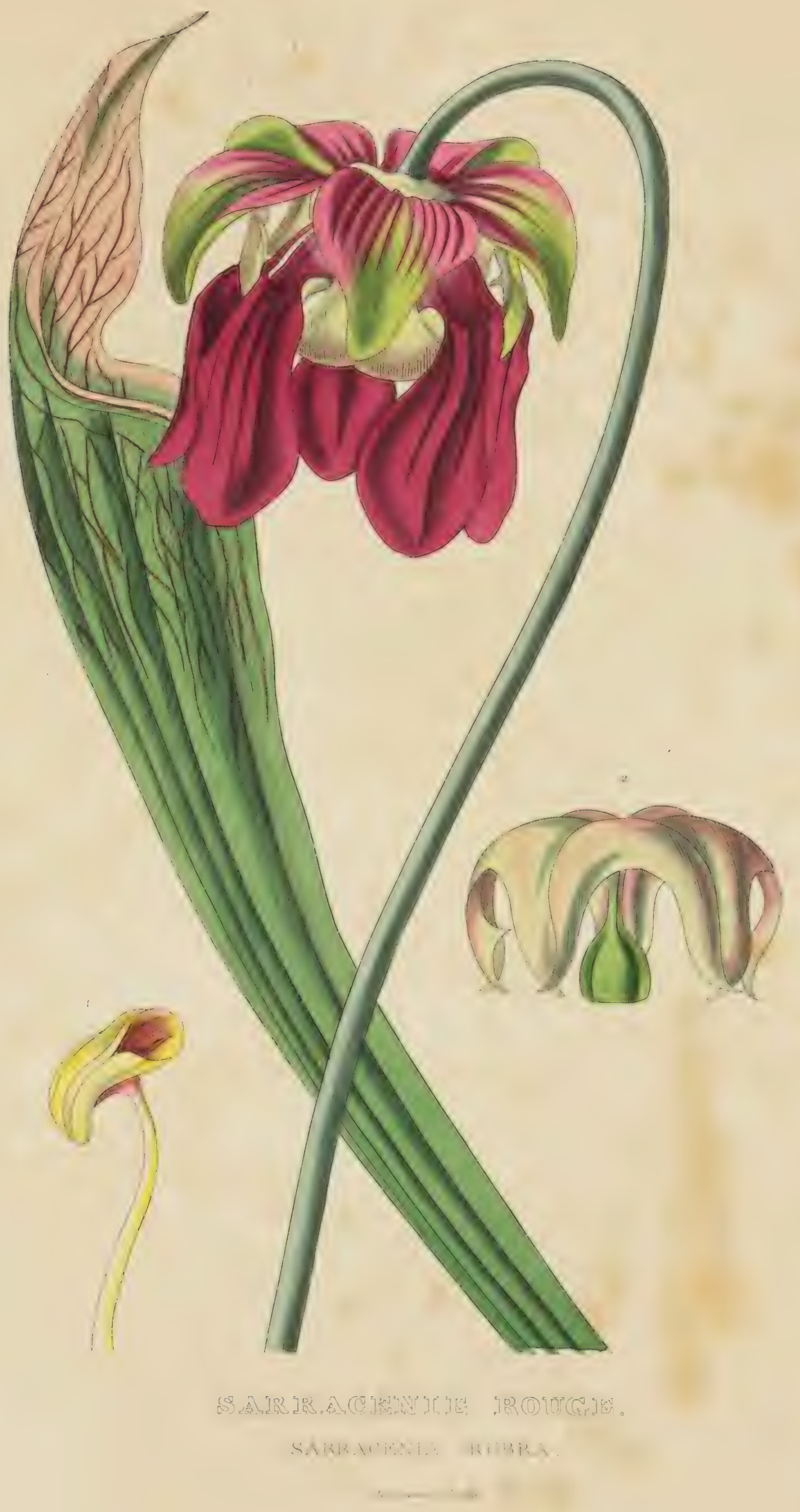




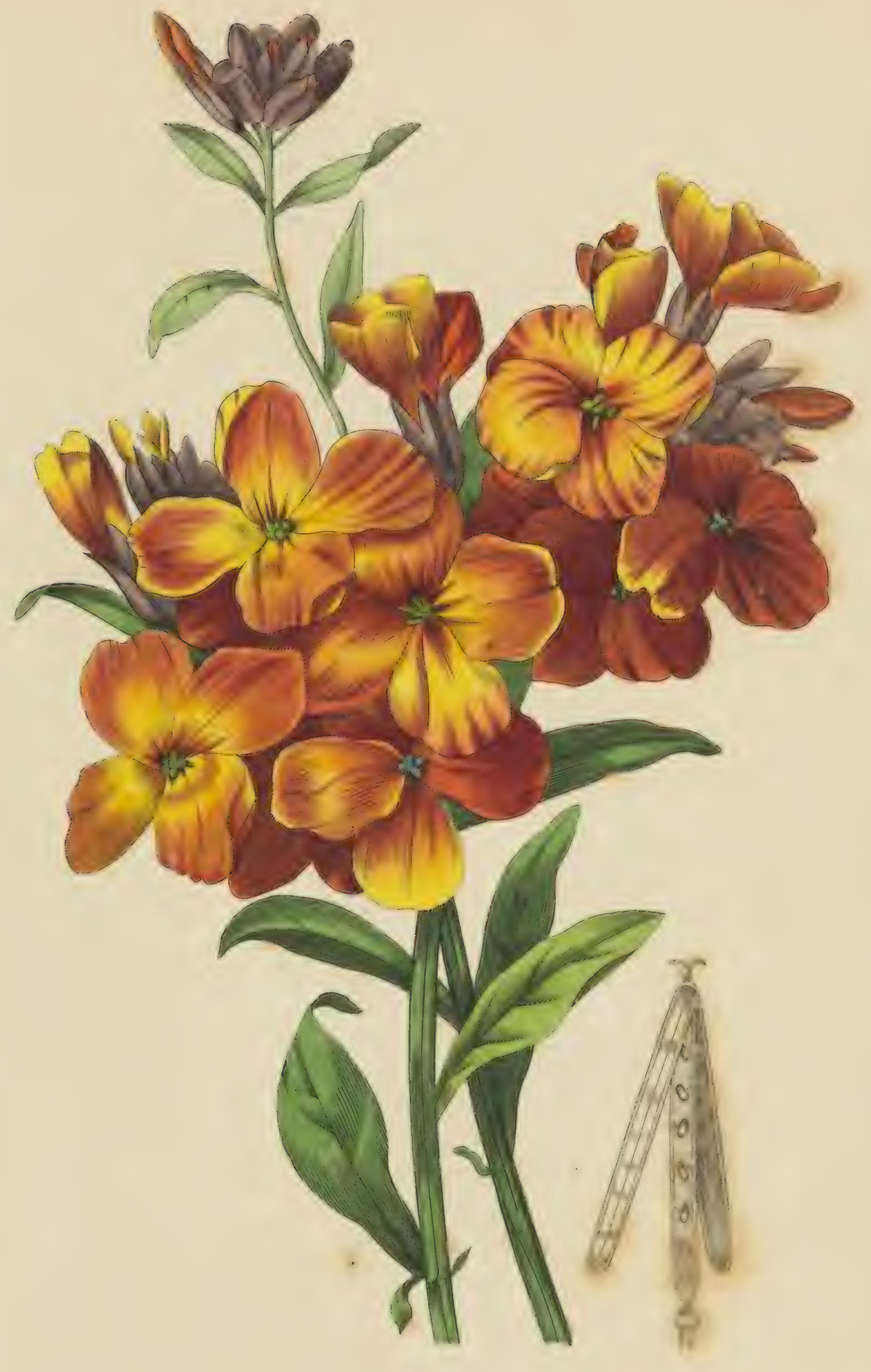

प्w4

GTROFTIFE VOHIER. 


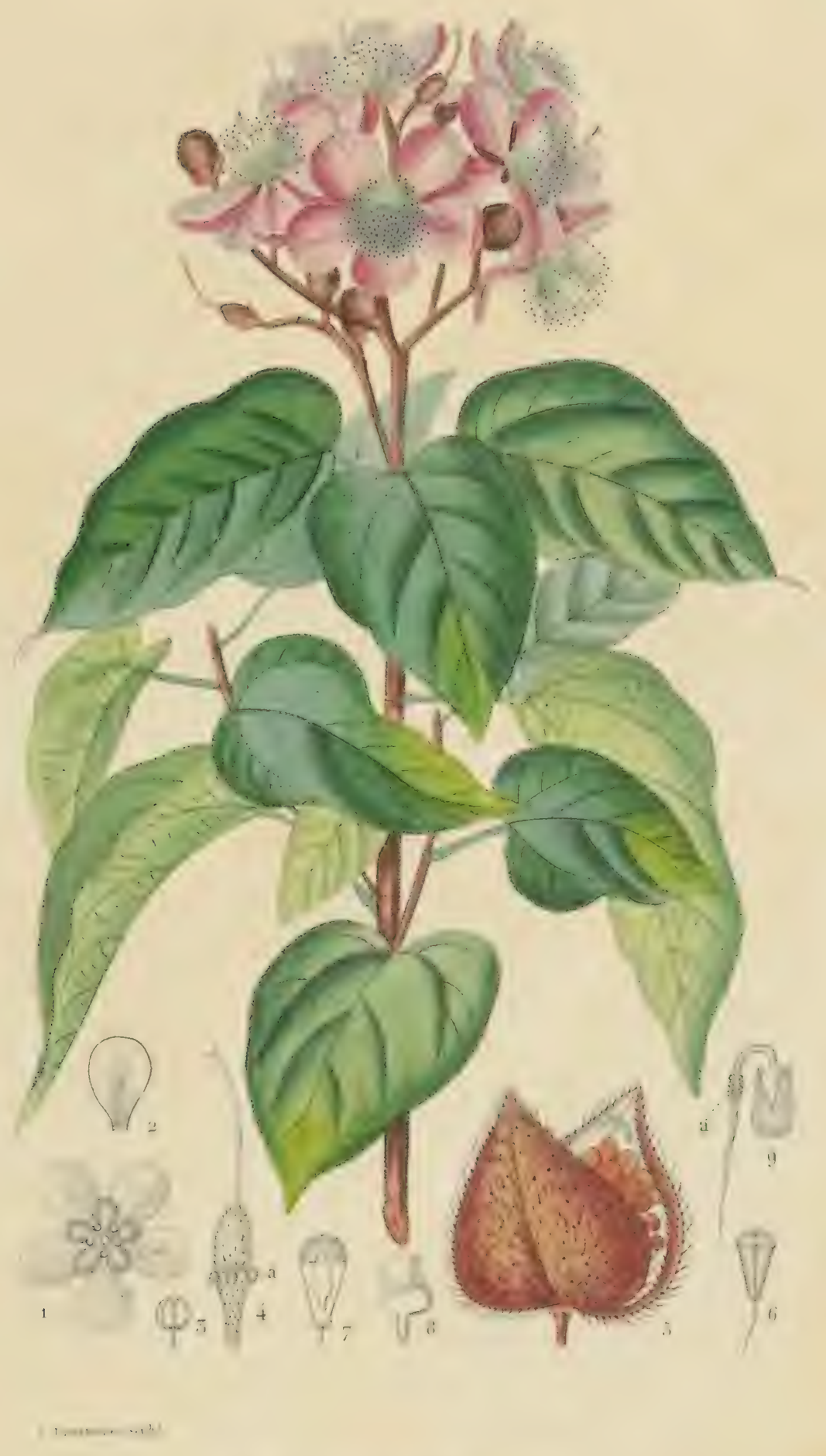

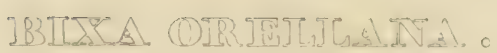

ROCOUIER CUITITE 


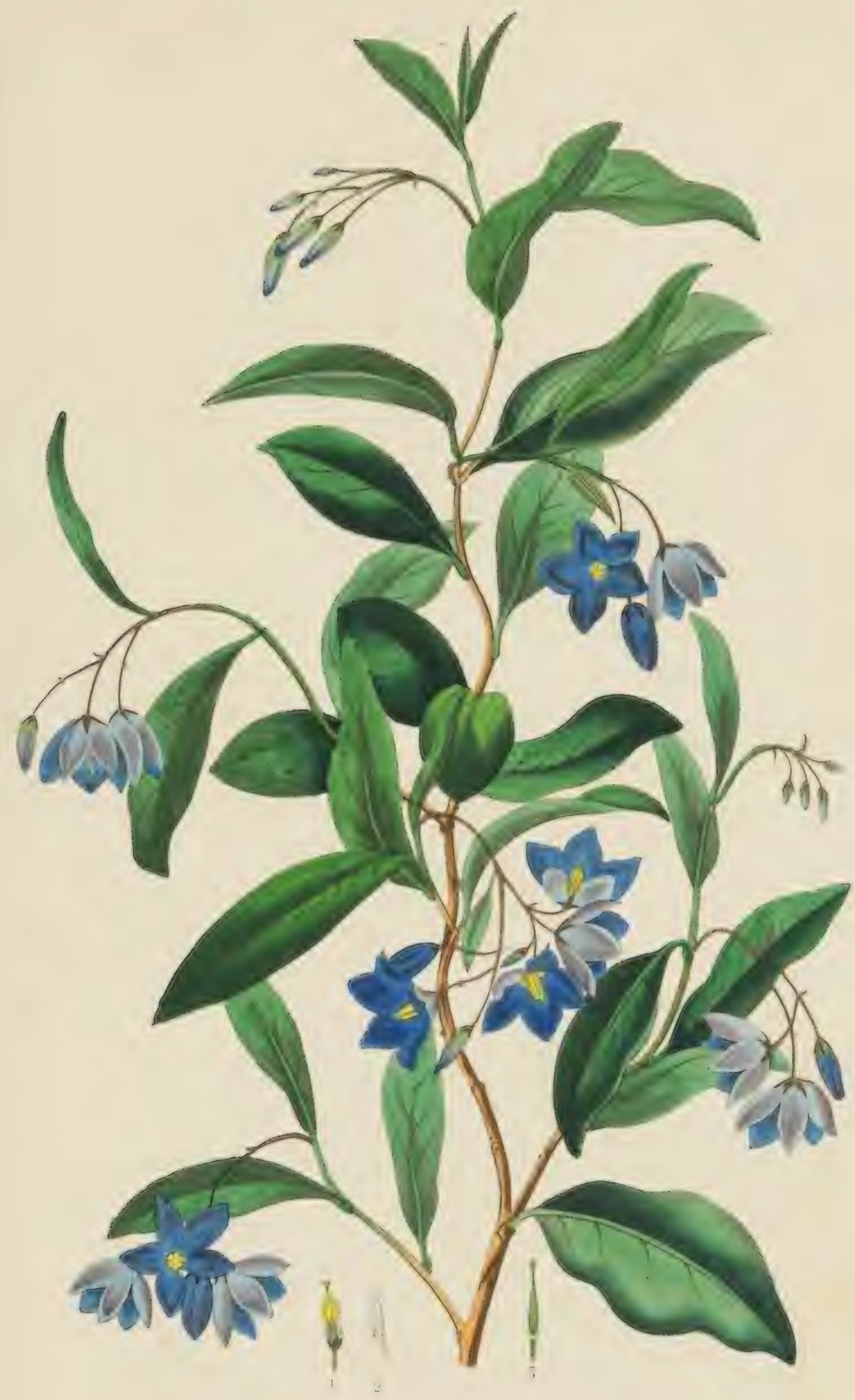

SOTTYE IETEROPIYLTE 


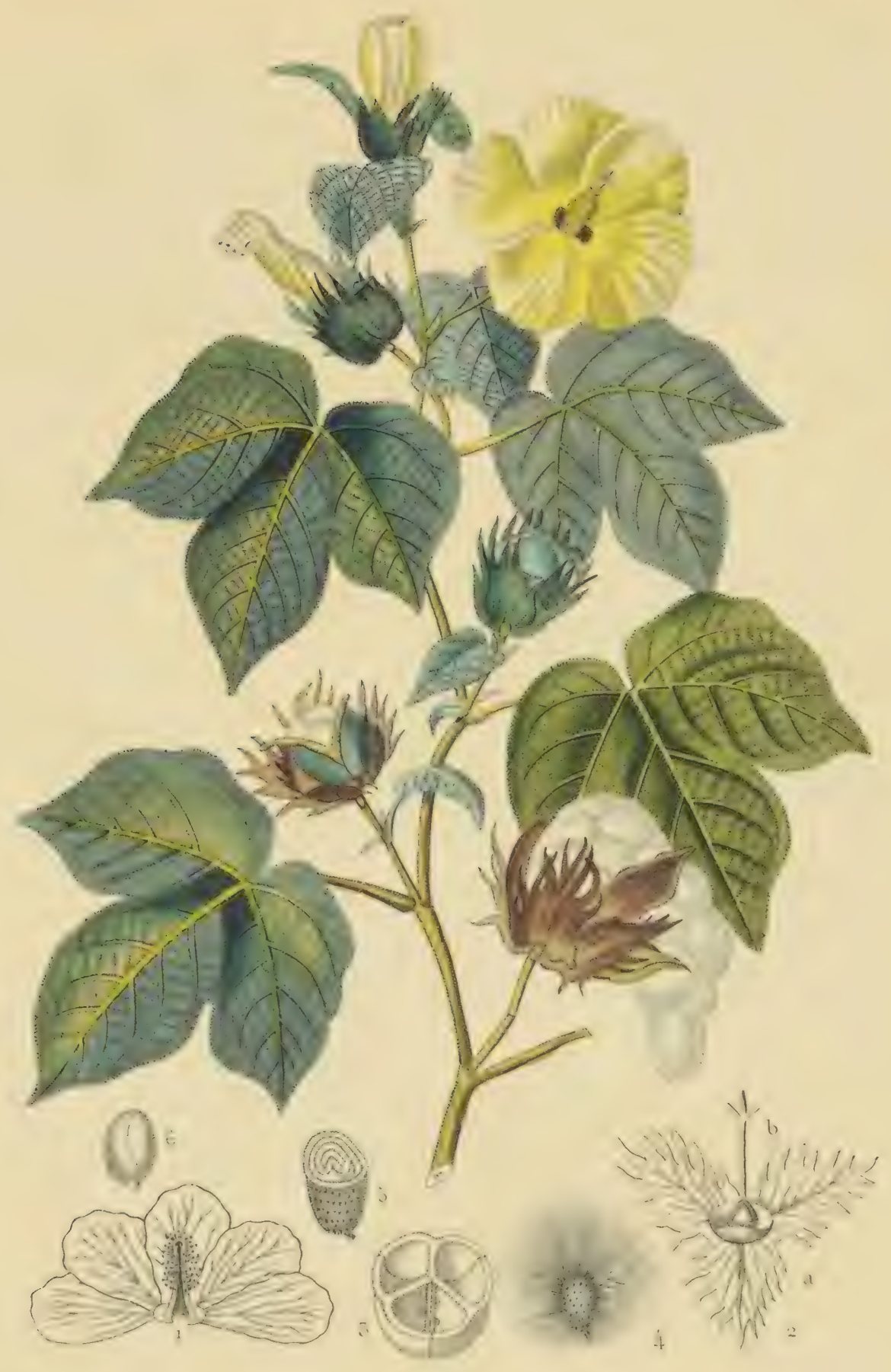

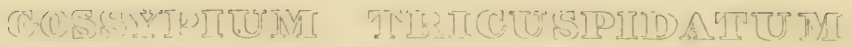
COTONNIER A TKOIS POTNTES 


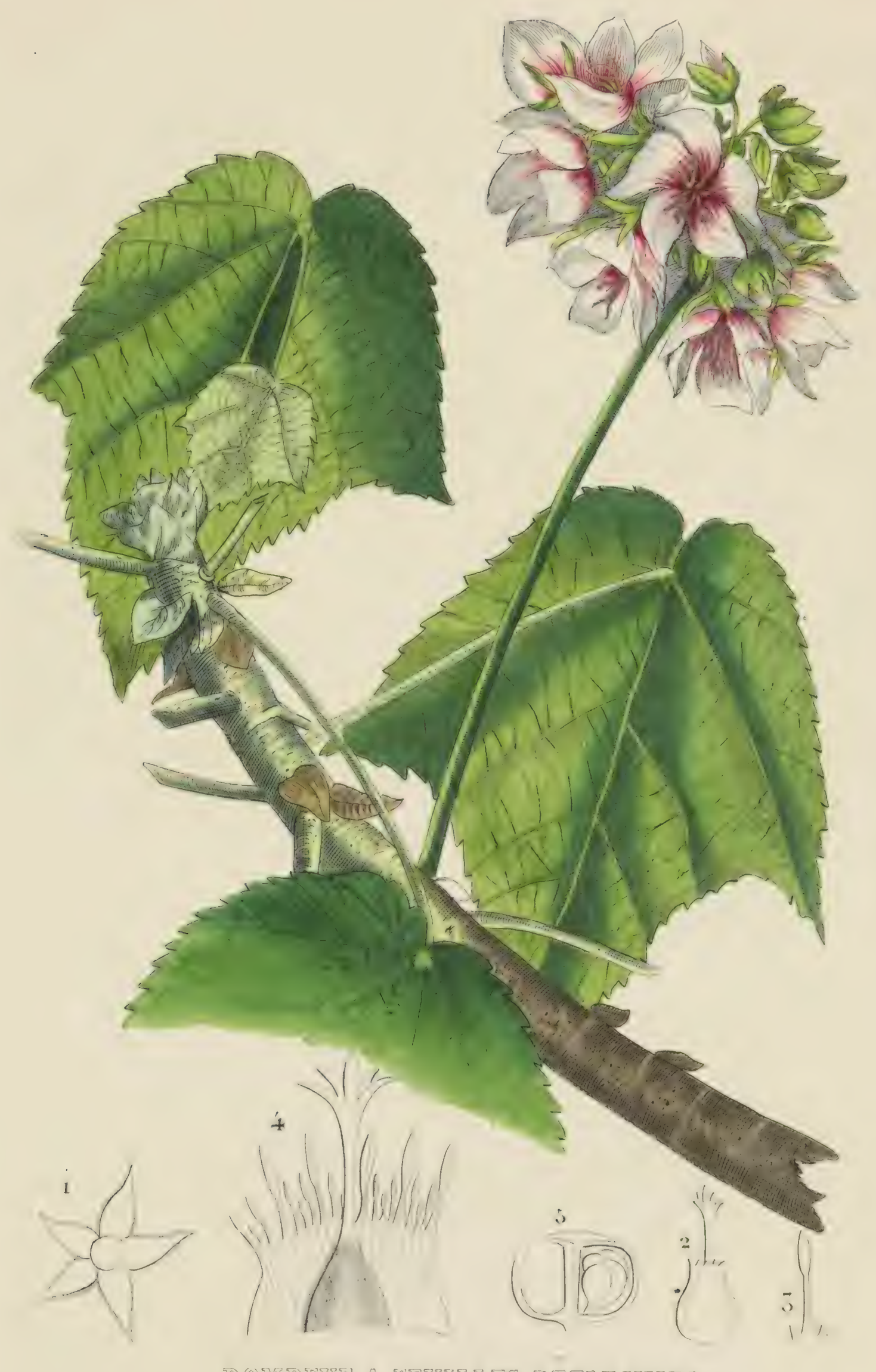

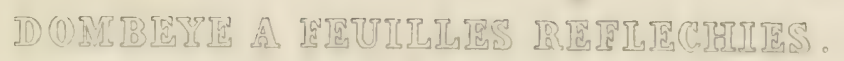

D) OMBETA REFLEXA .

1. C'alice.2.Pistil. 3.Etamine 4. Pistil et étamines grossis. 5.0vaire. 


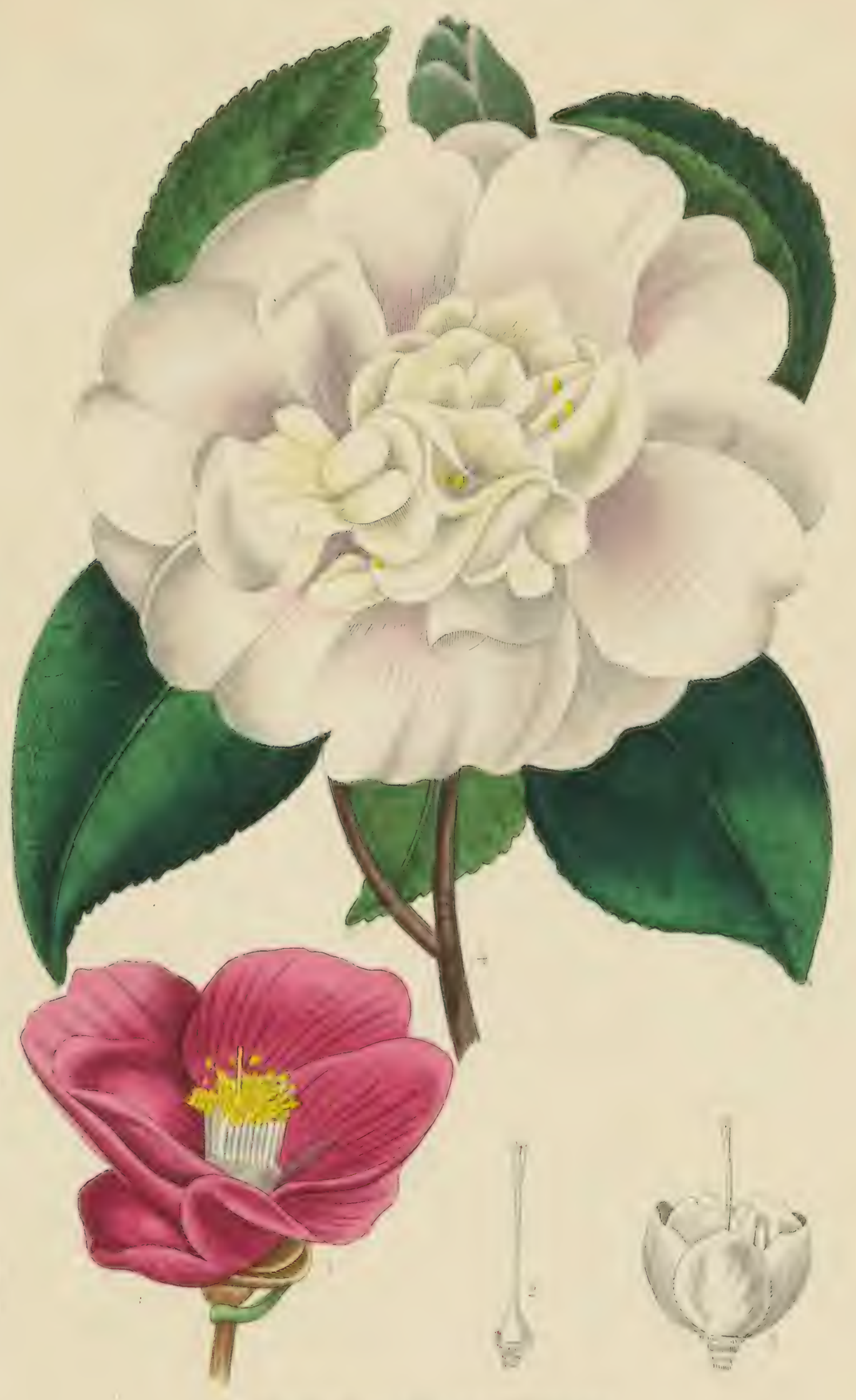

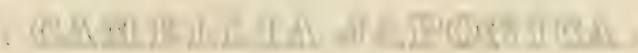

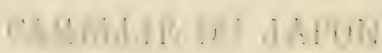

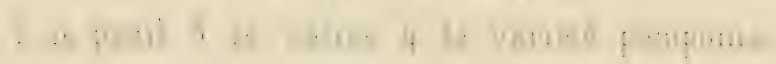




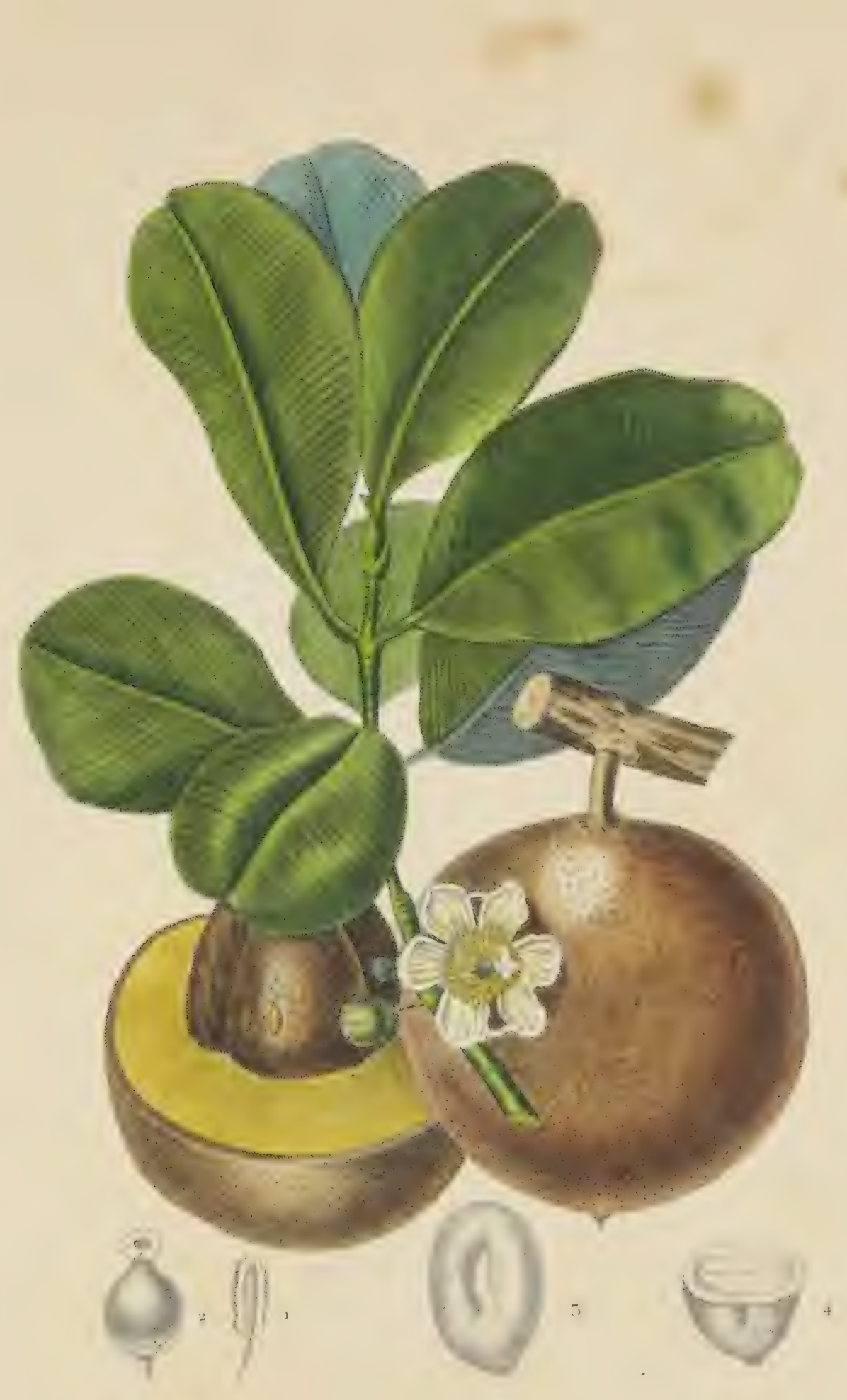

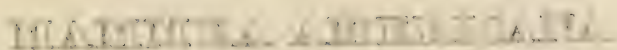

MA.MEI D'AMÉRIQUE. 


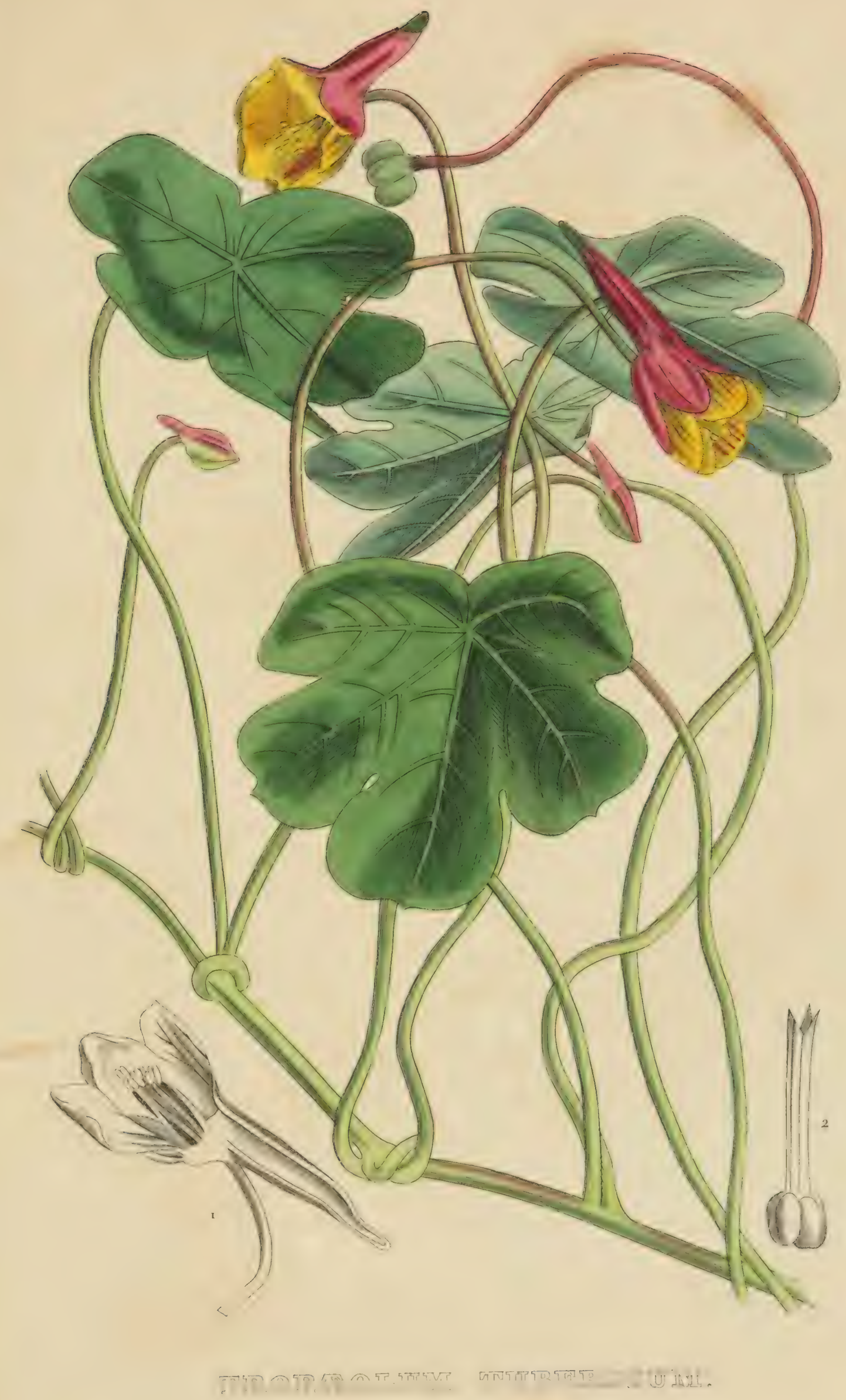

CAPUCTNE TUBFEREUSE 


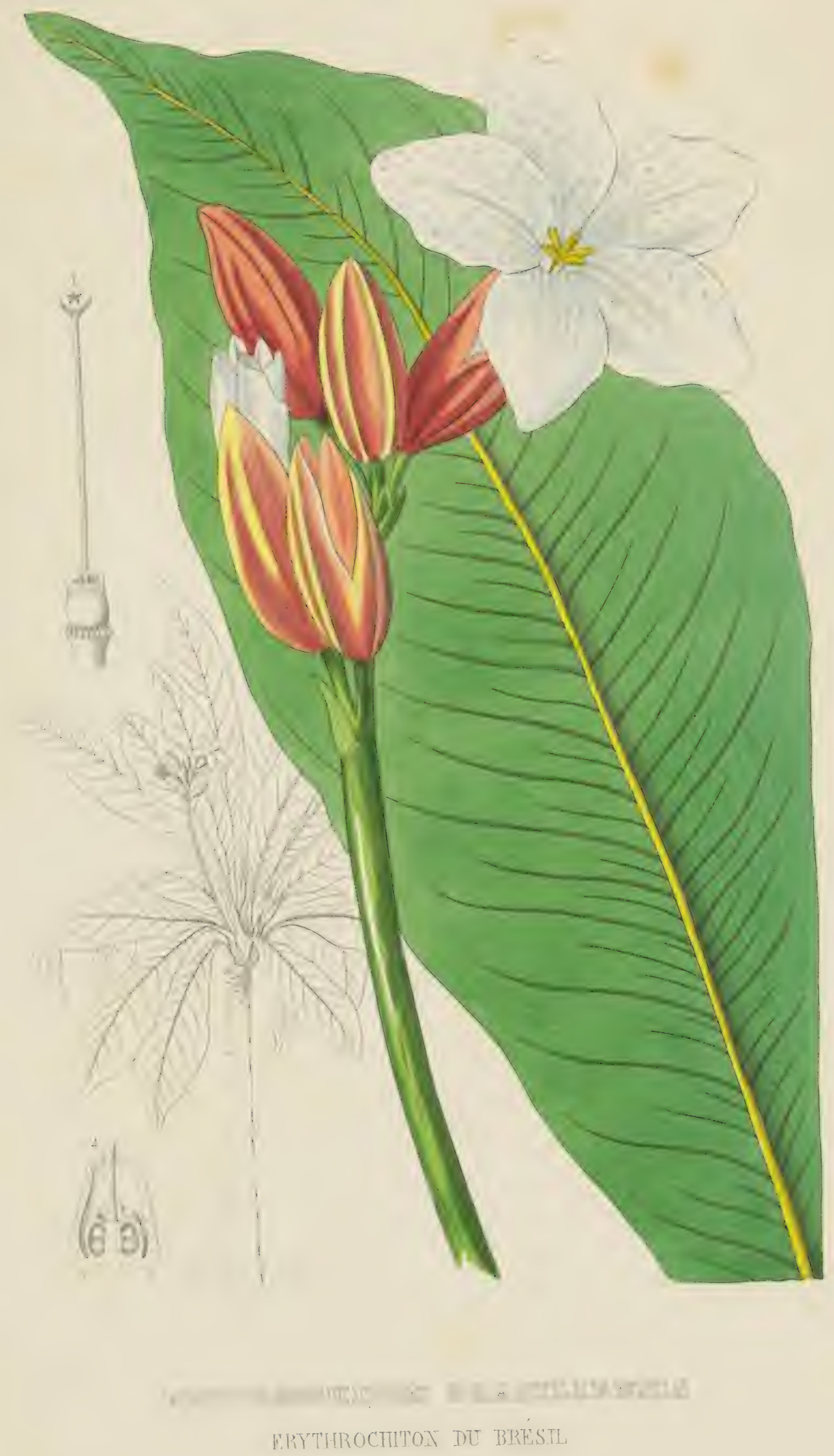




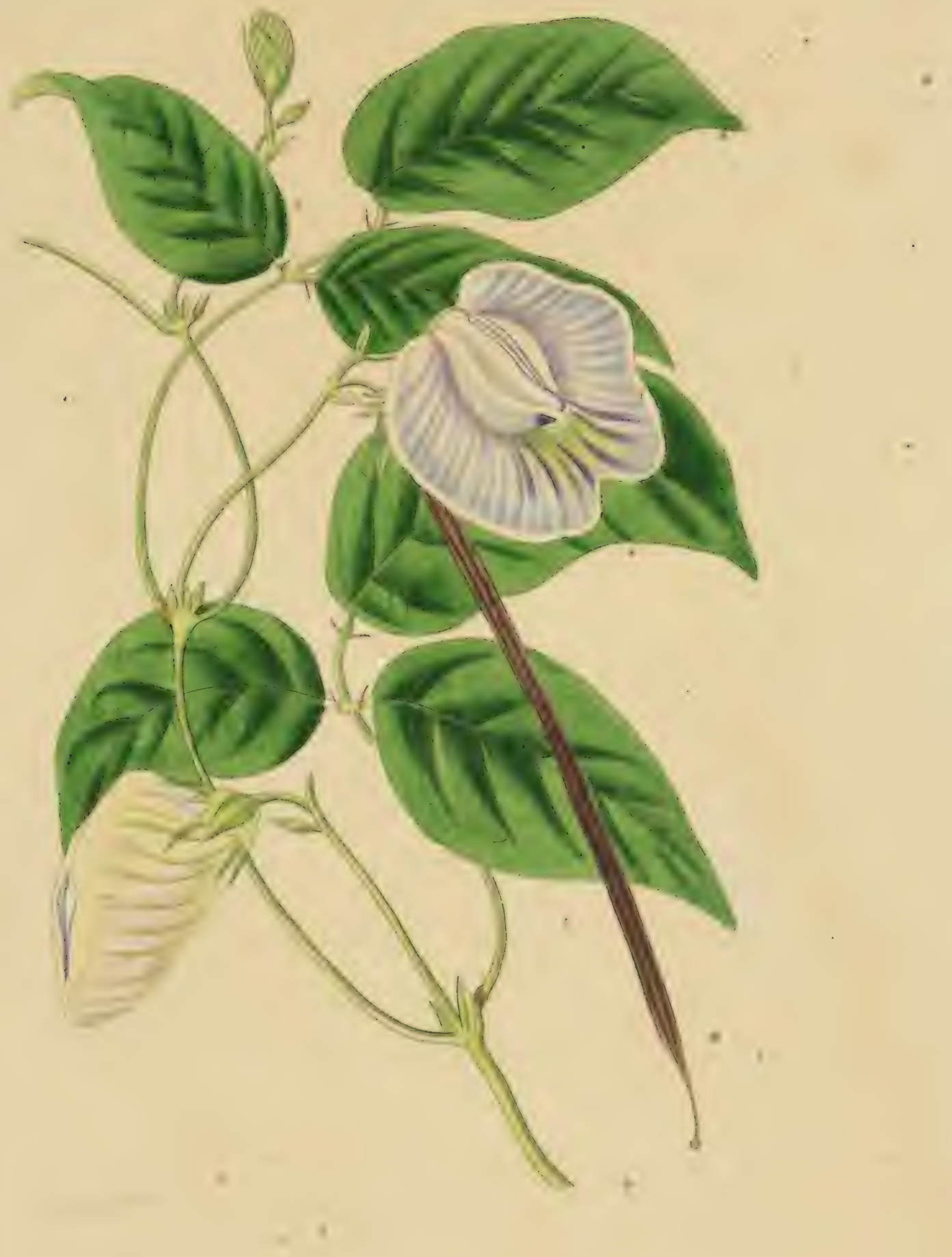

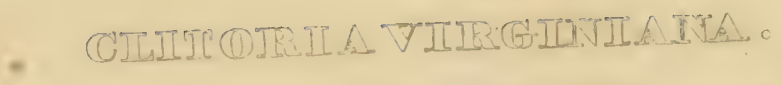
CLTIORE DE VIRGINIE. - 


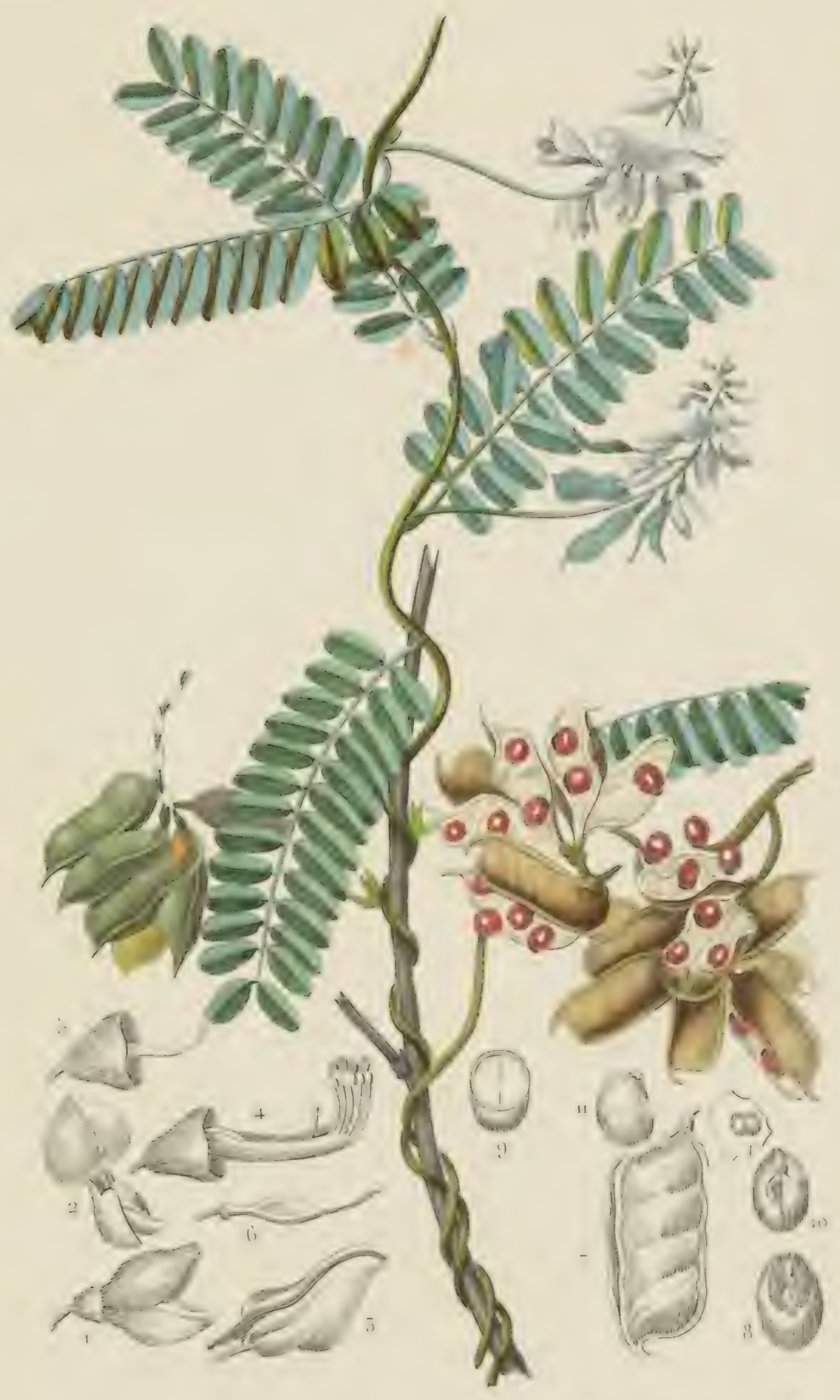

T.TANE:A REQHISSE 

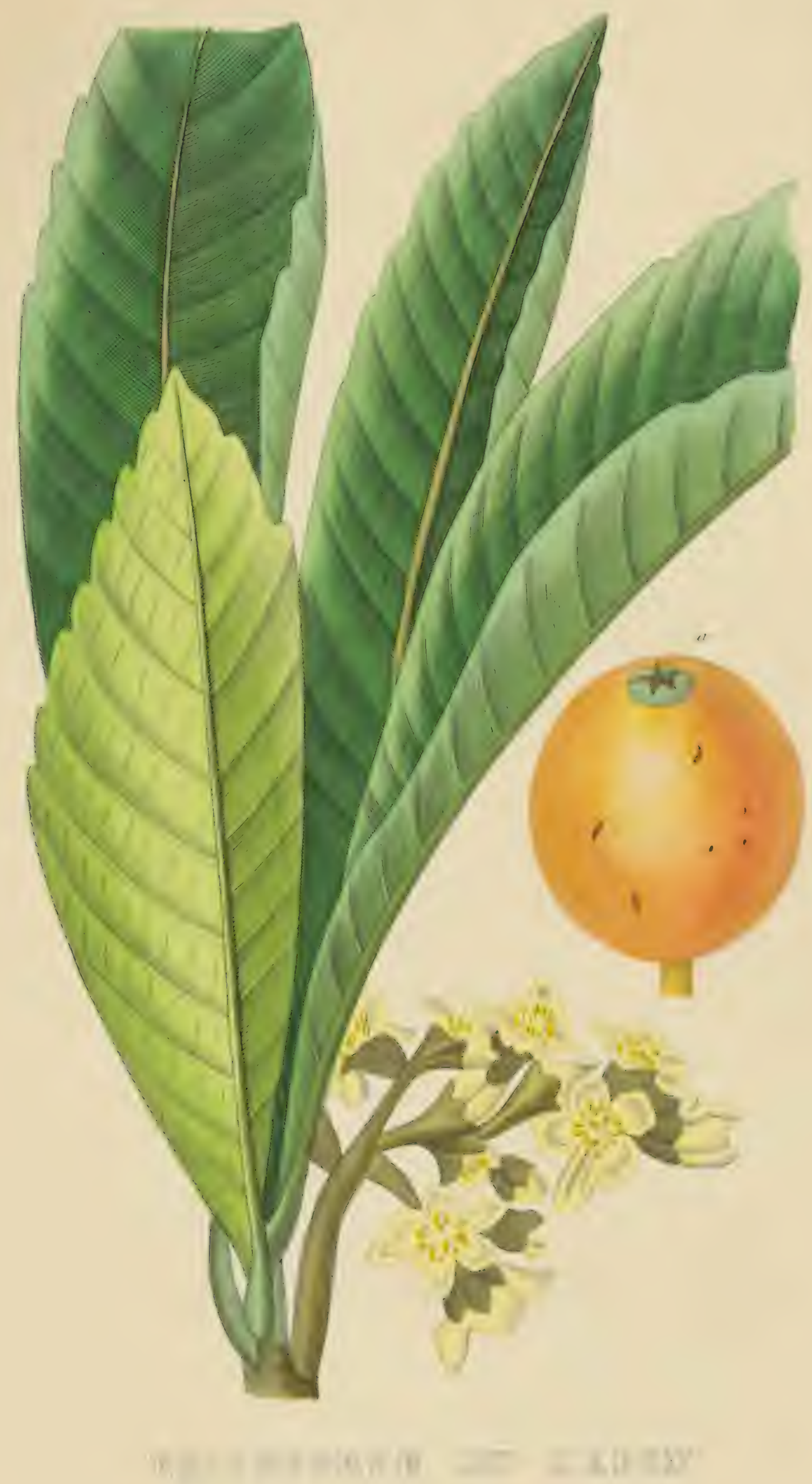

ERTOBOTRYA JAPUI: 


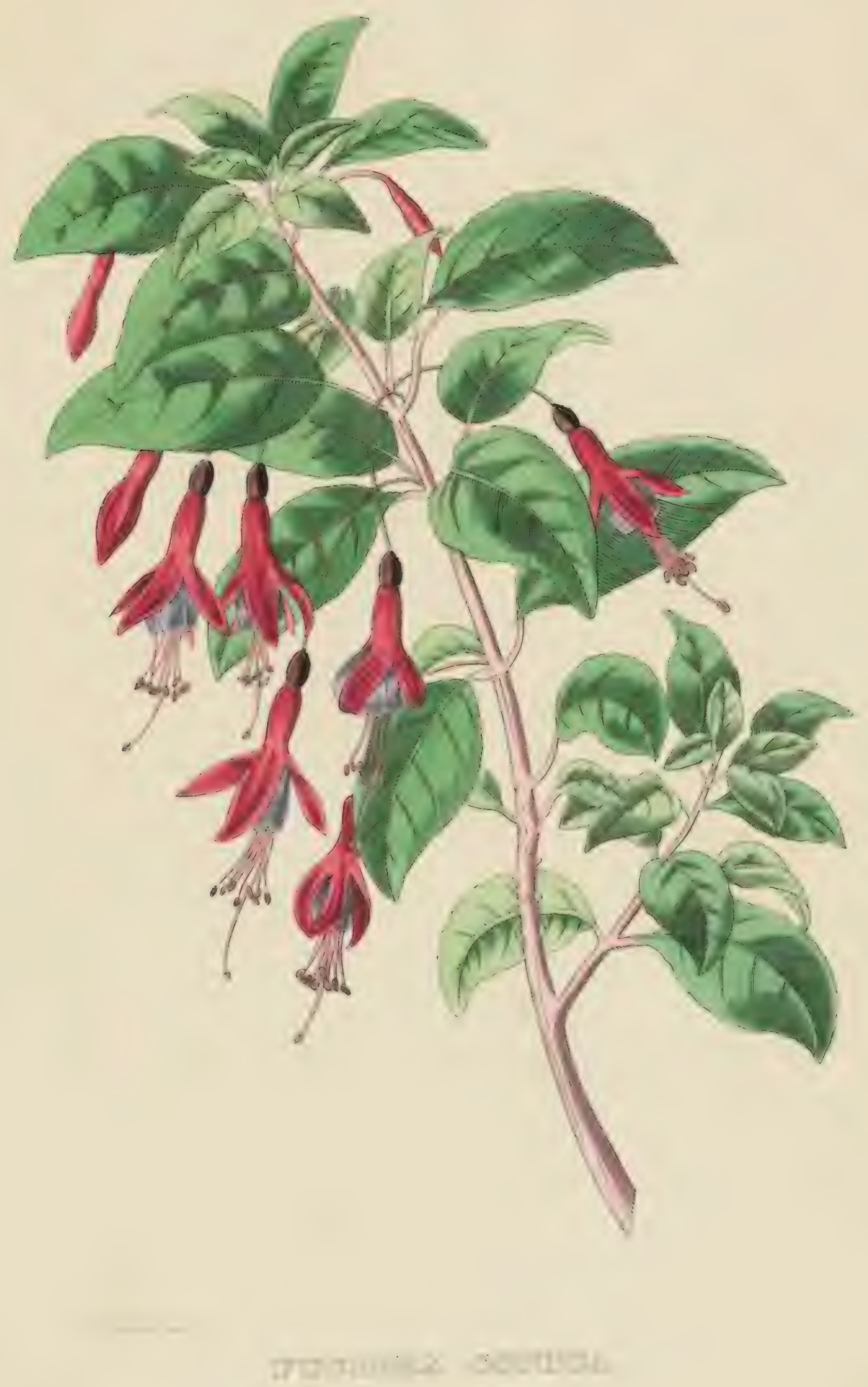

FUCHSHE CONTOUK . 


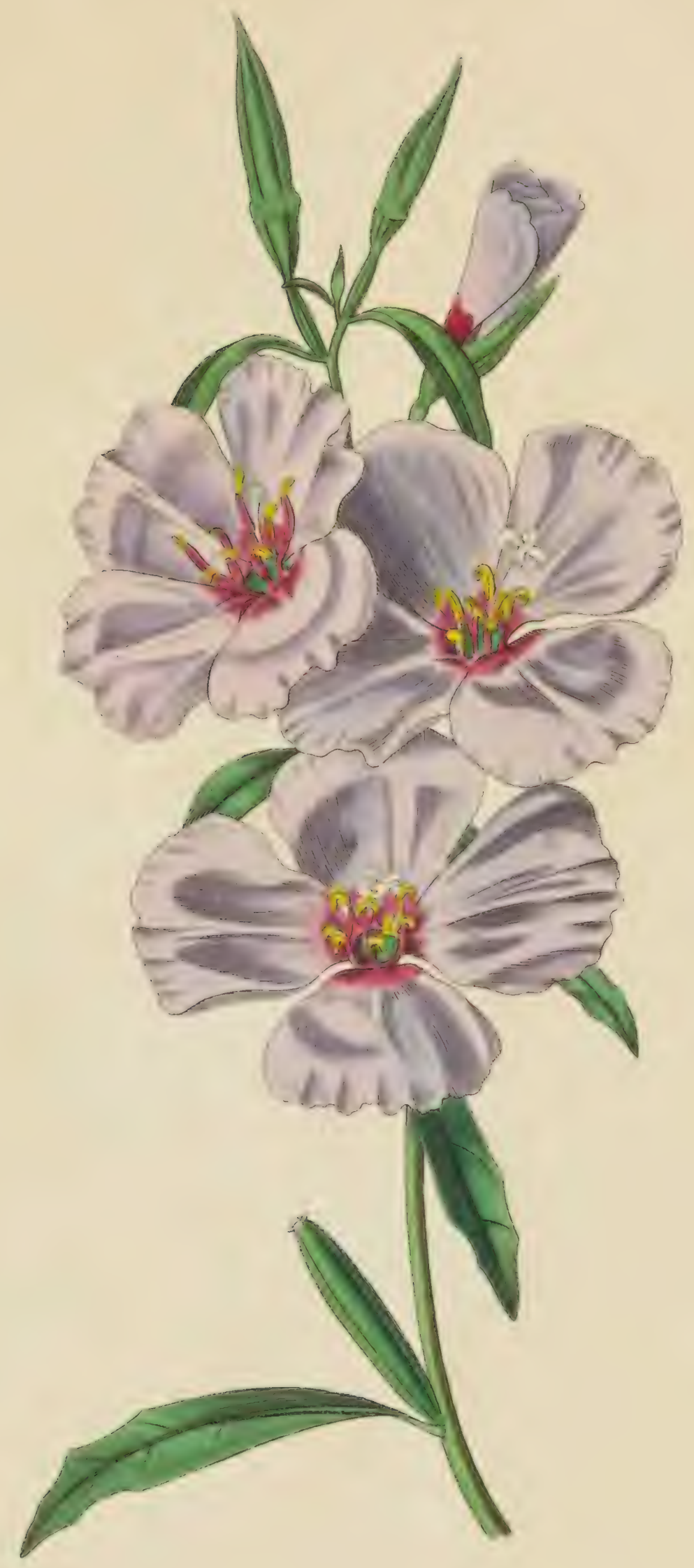

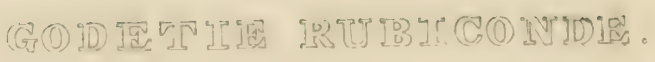

G () HIUA RUBICUNDA. 


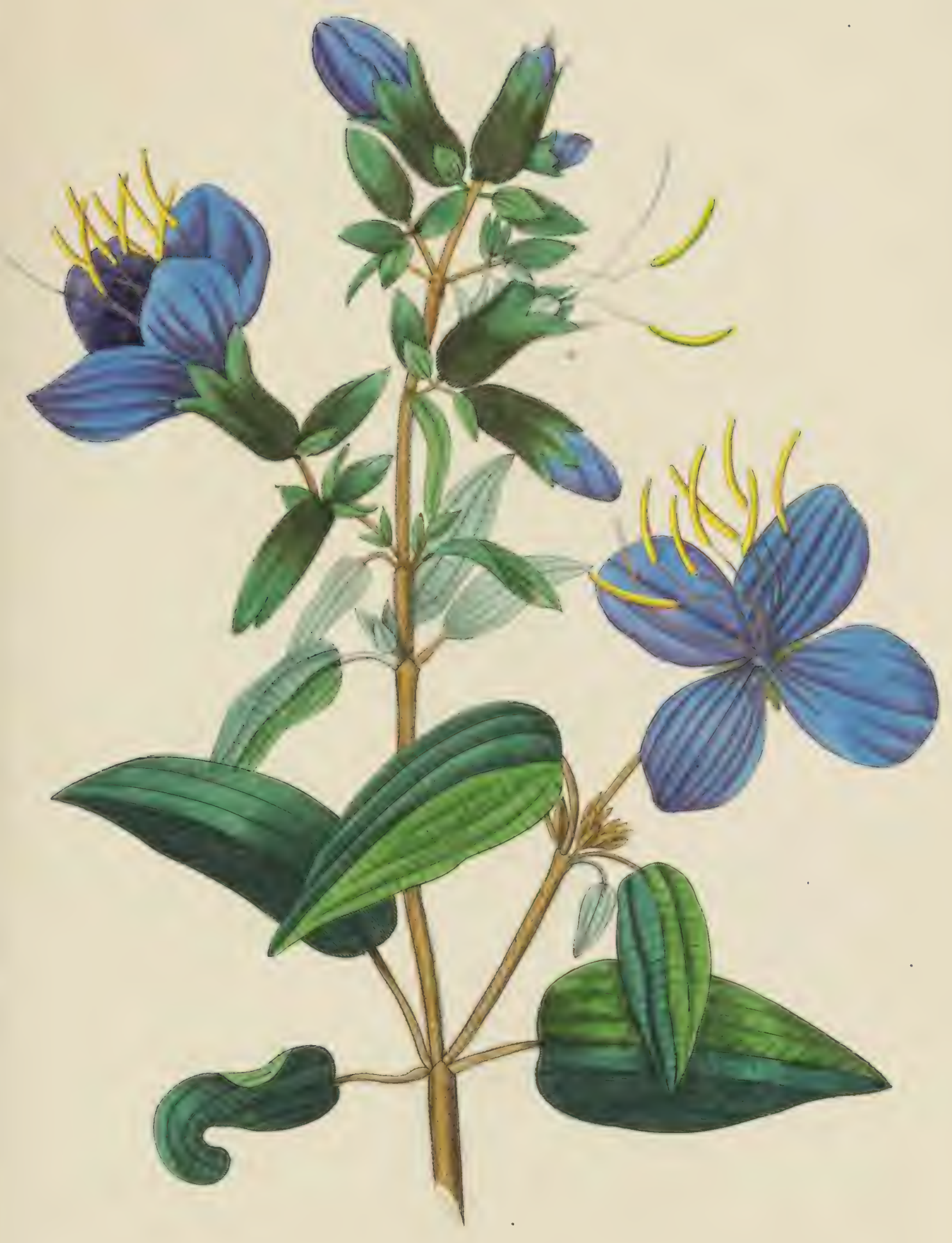

\section{TI}

RHEXIA PRTNCEPS . 


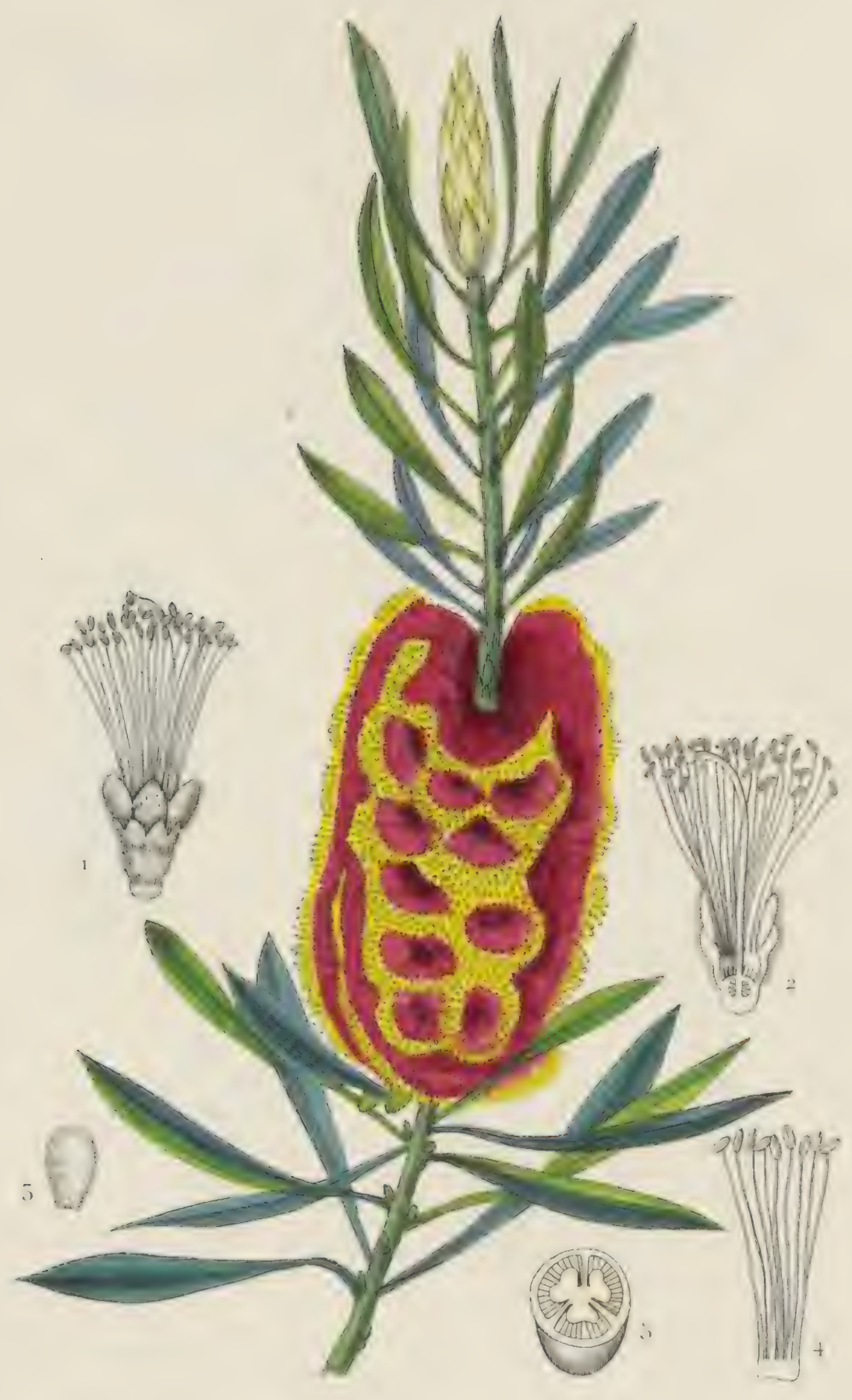

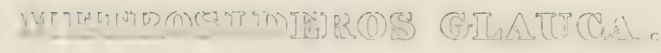

MF́TROSIDEROS GTALOUE. 


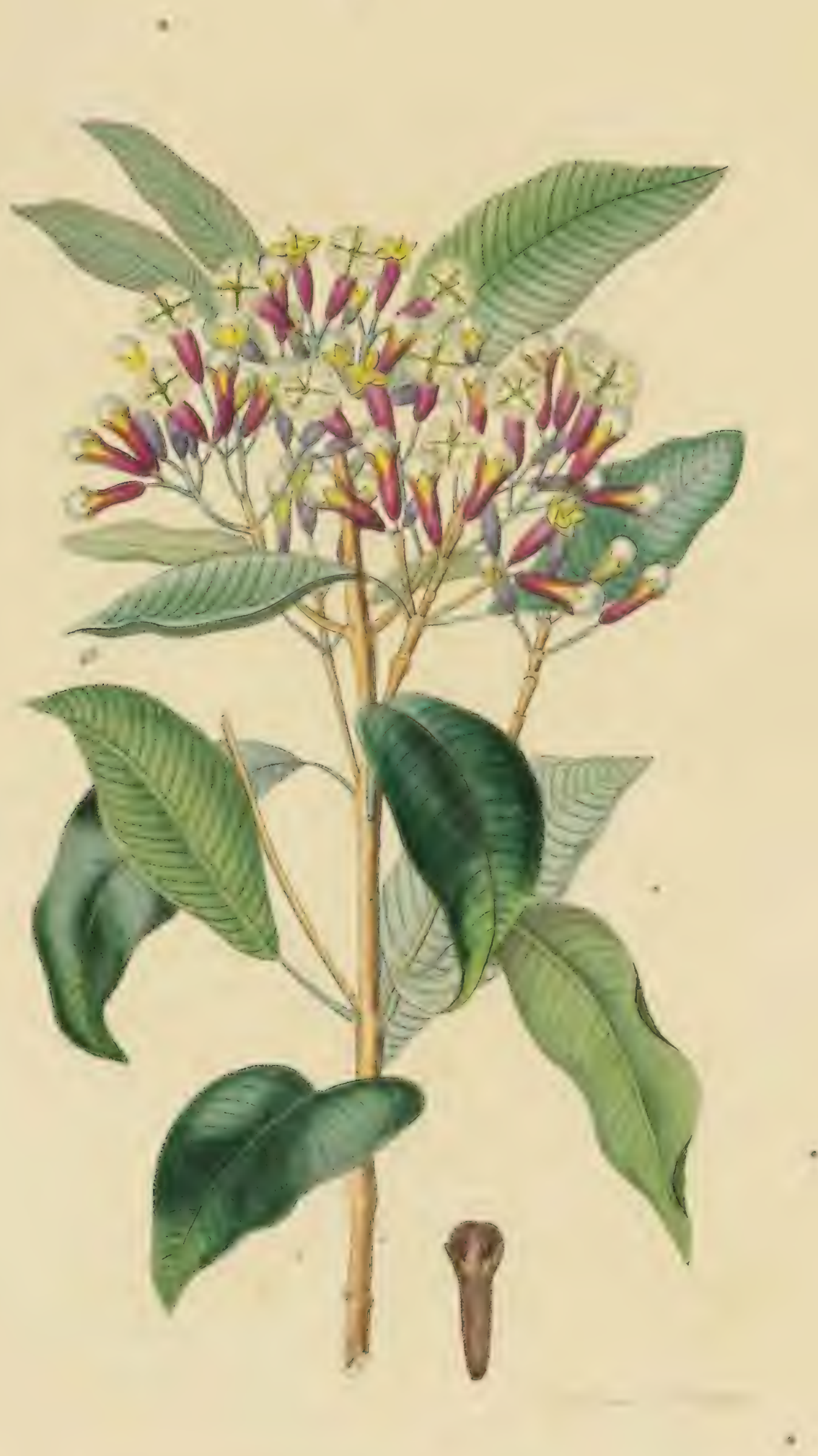

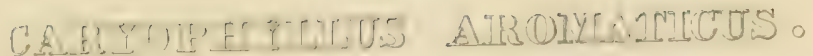

GIR(OFIALR AROMATTOUE. 

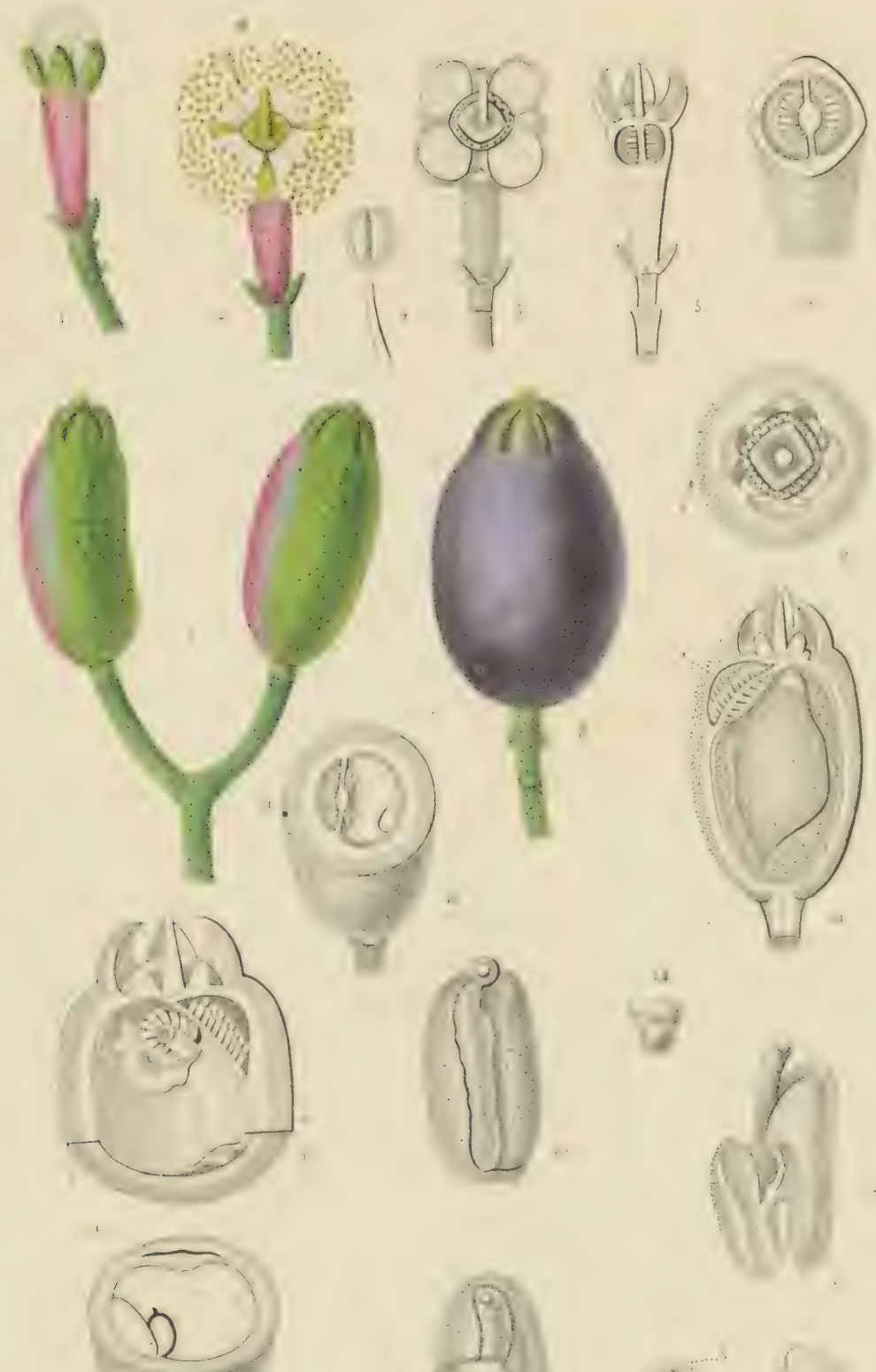

$\exists$
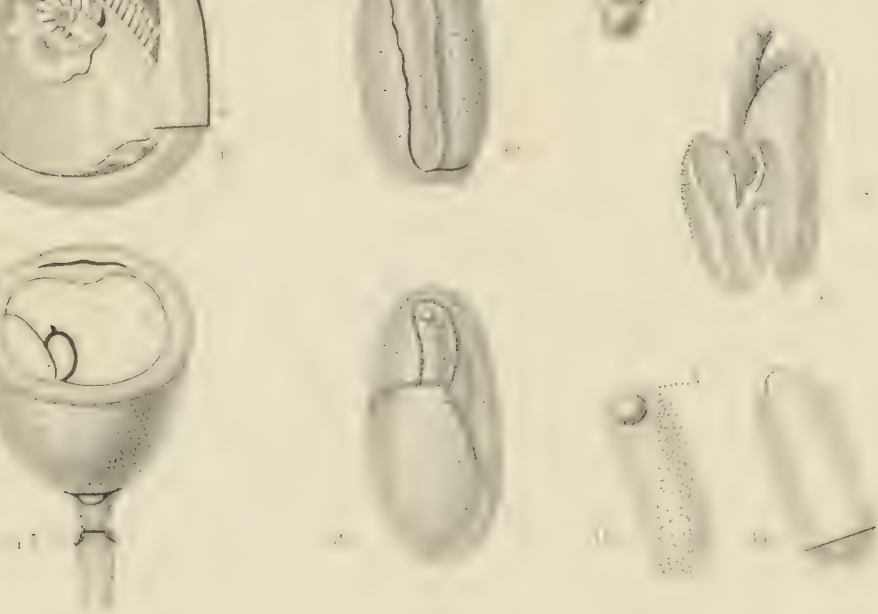

-. e de la fleur , du frus

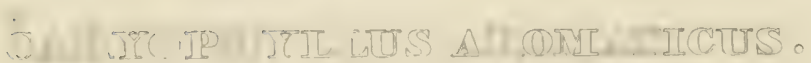




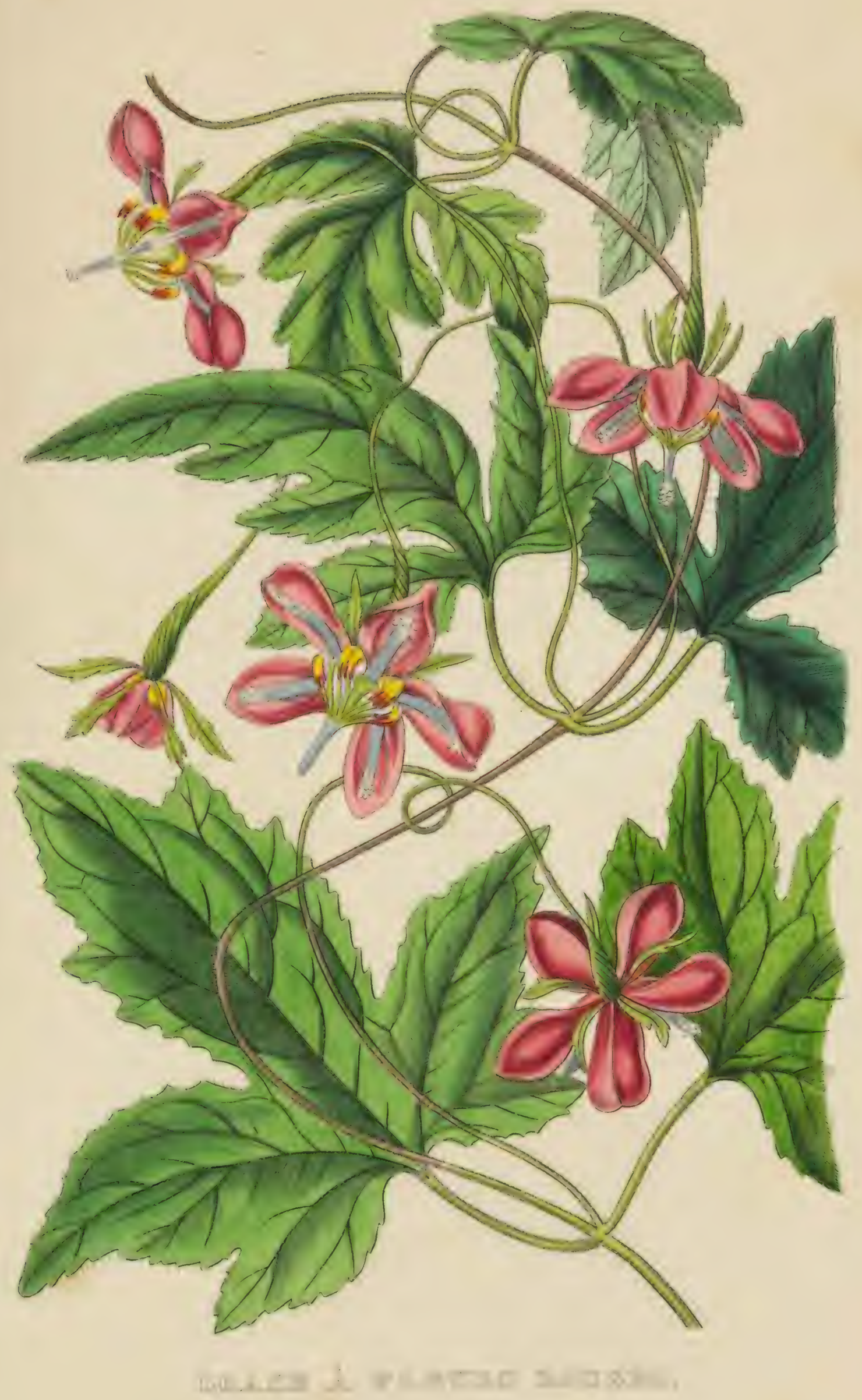

IOOASA 1.THEITE. 


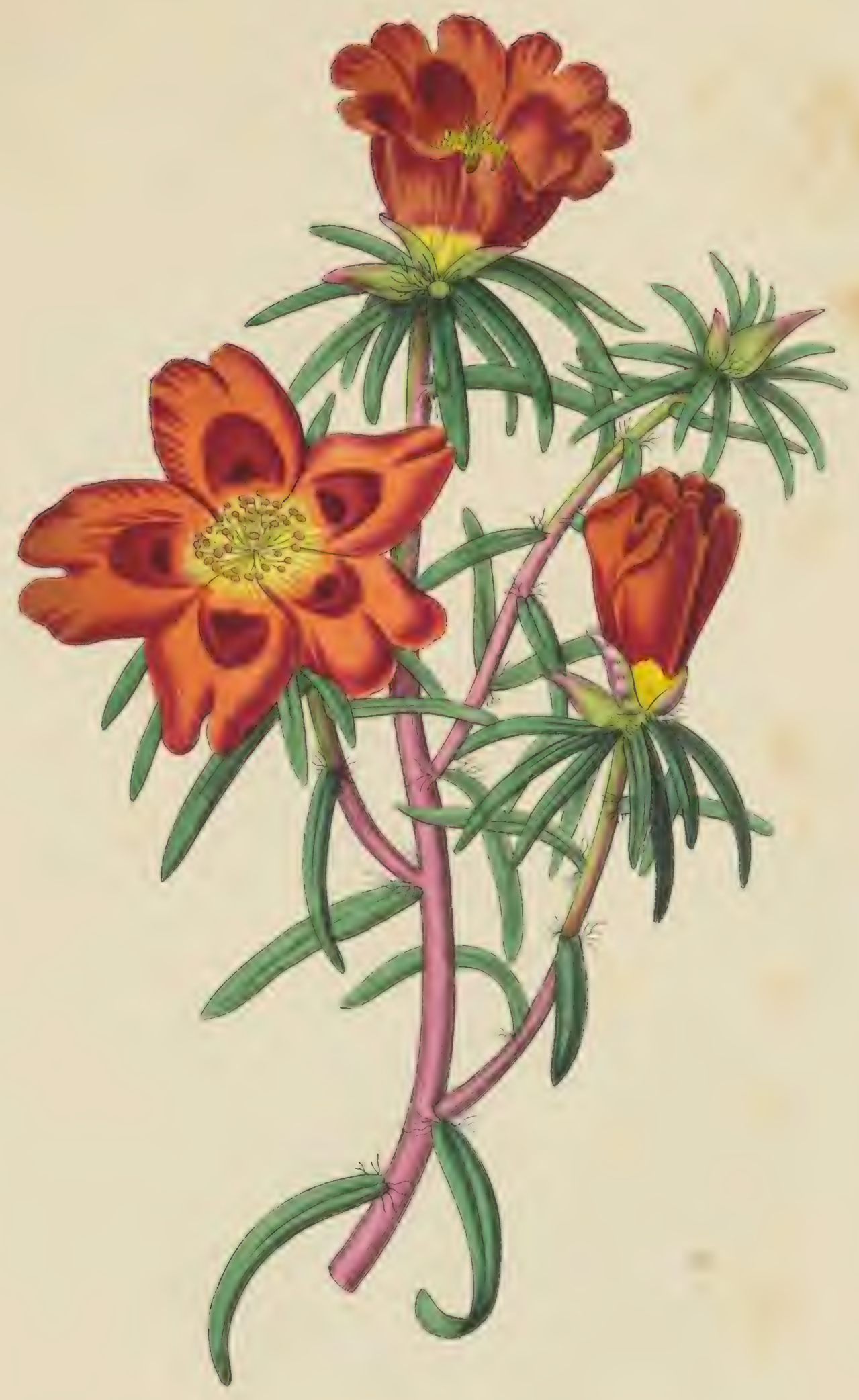

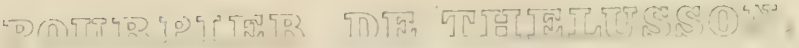

PORTUTAACA THELUSSONII. 


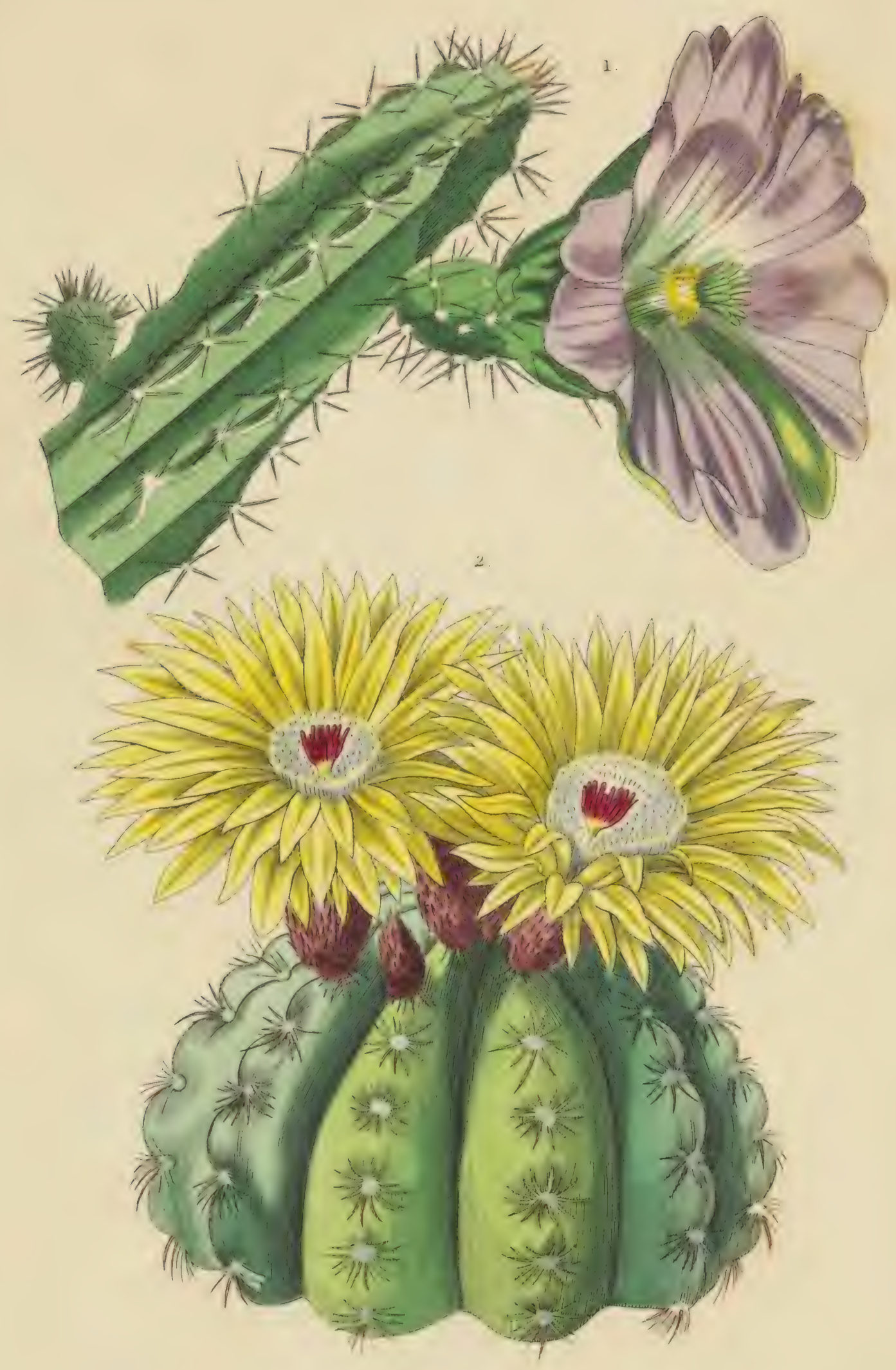

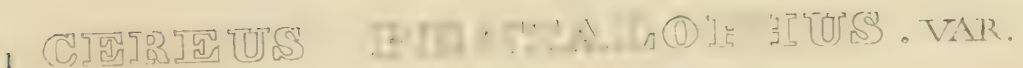

CIFIREE A CLNO ATIES.

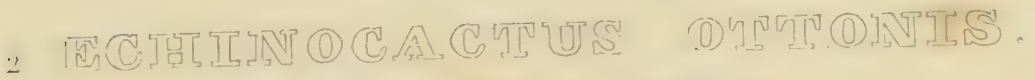

HCHINOCAC'TE D'OTTON. 


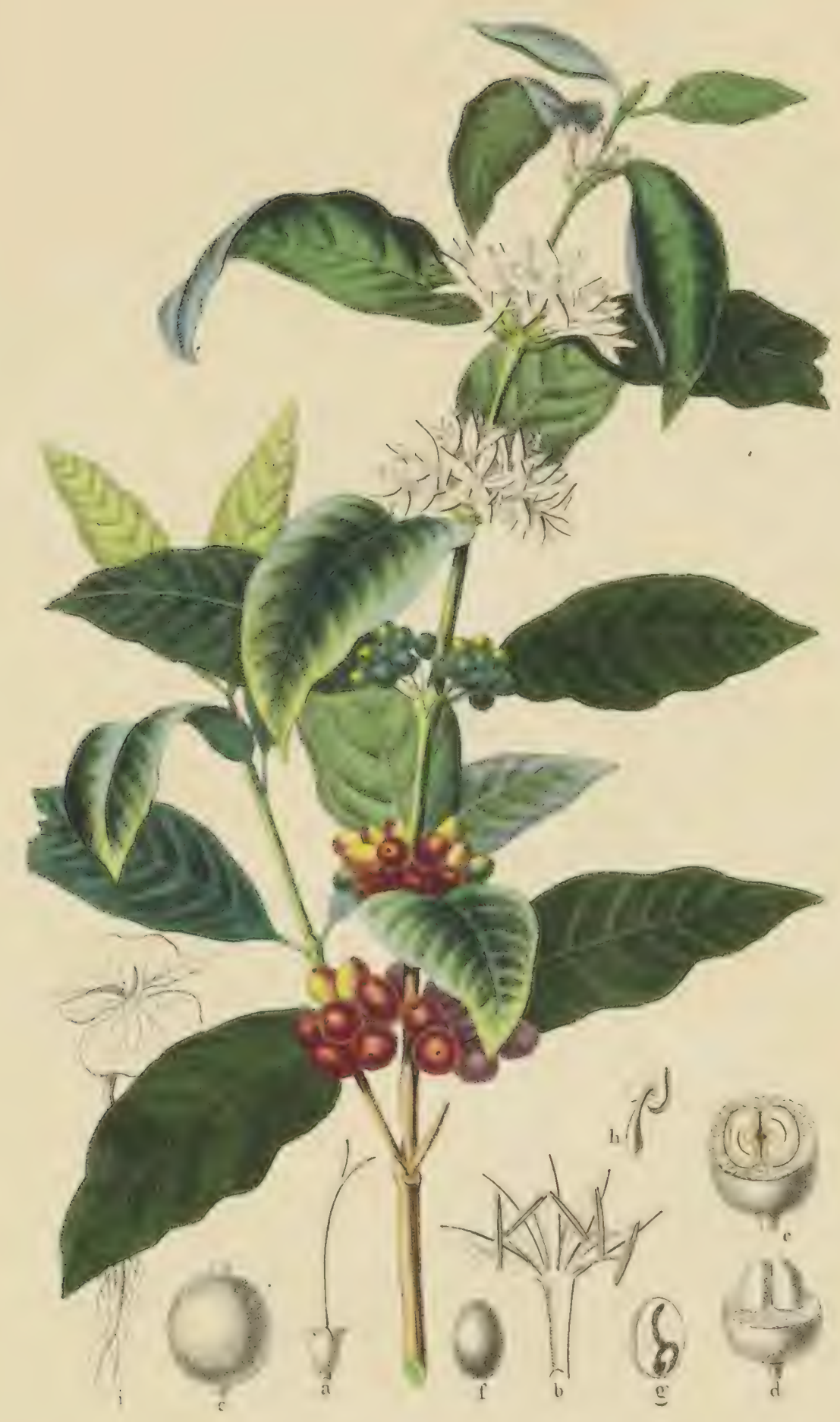

T(D)IETTEA AIRATBICA.

CAFE' D'ARABIE. 


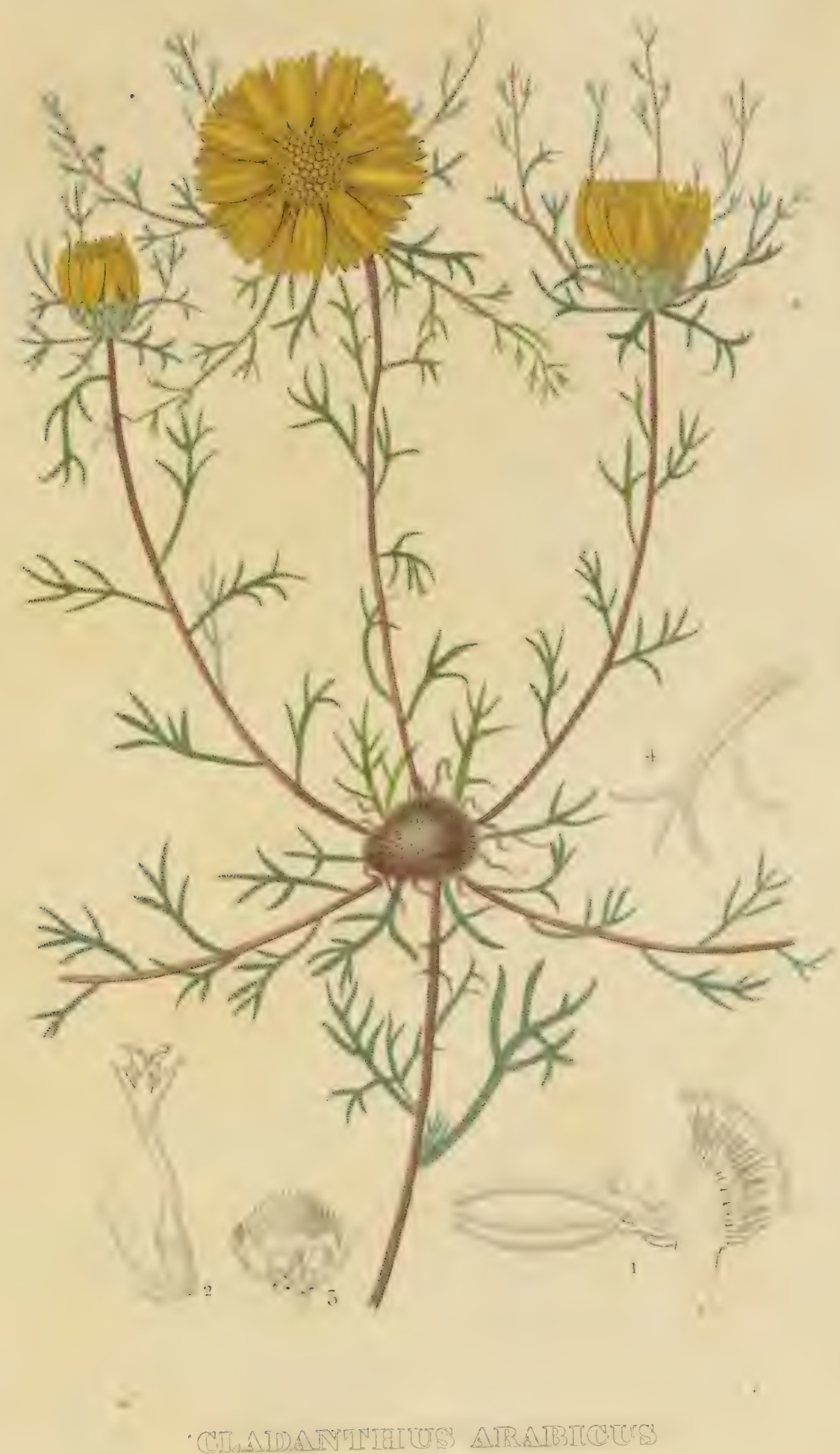

CI.ADANTHE: D'ARABLE. 


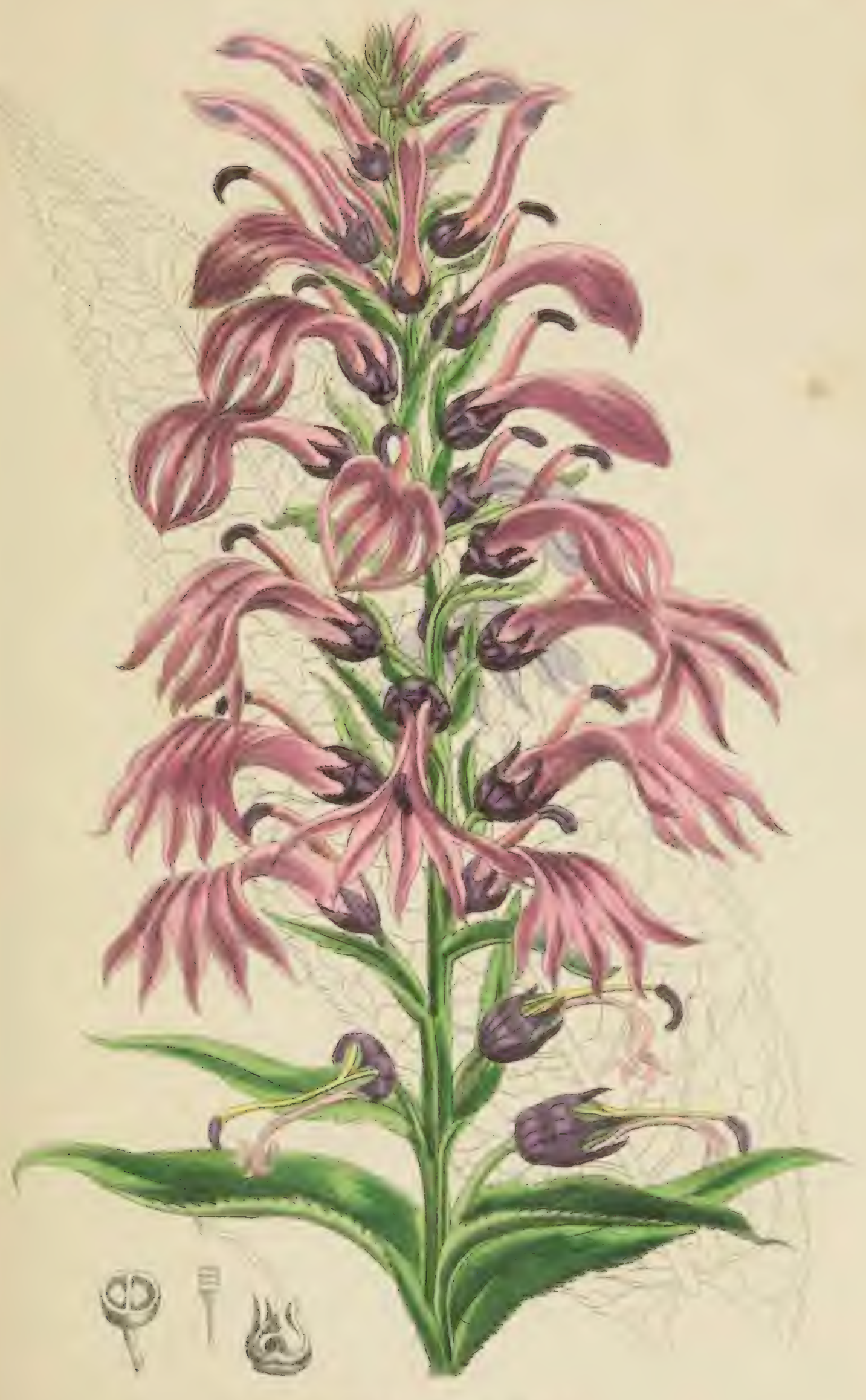

TUIPA BUANDA

TUPA A FLEURS ROSES. 


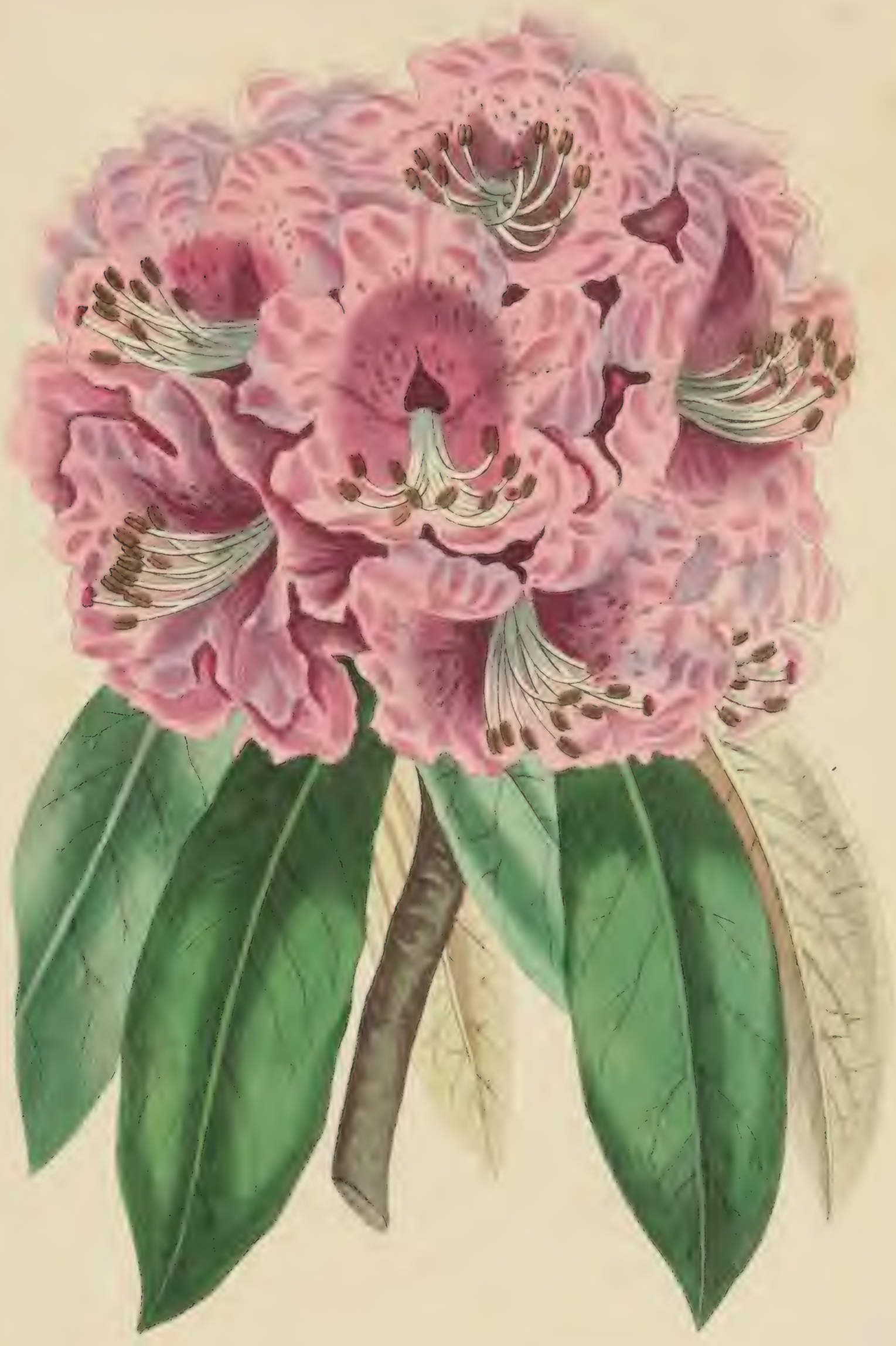

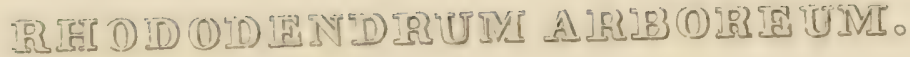

IROSAGF TIN ARIBRE. 


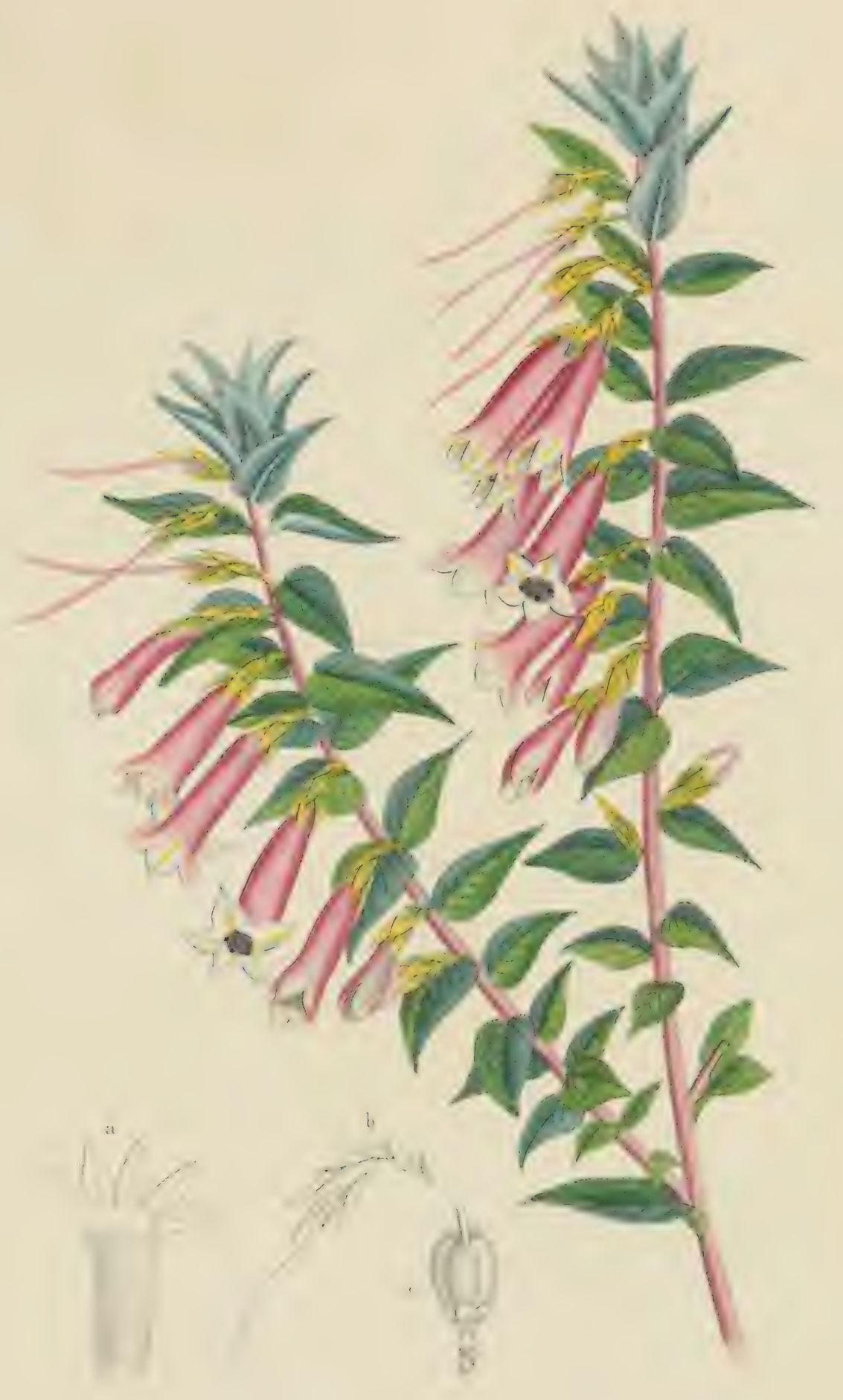

JEIPNCISISS GIBLANIDIE-IEILOJBLA

FPACRIS A GRANDES-TLEURS 


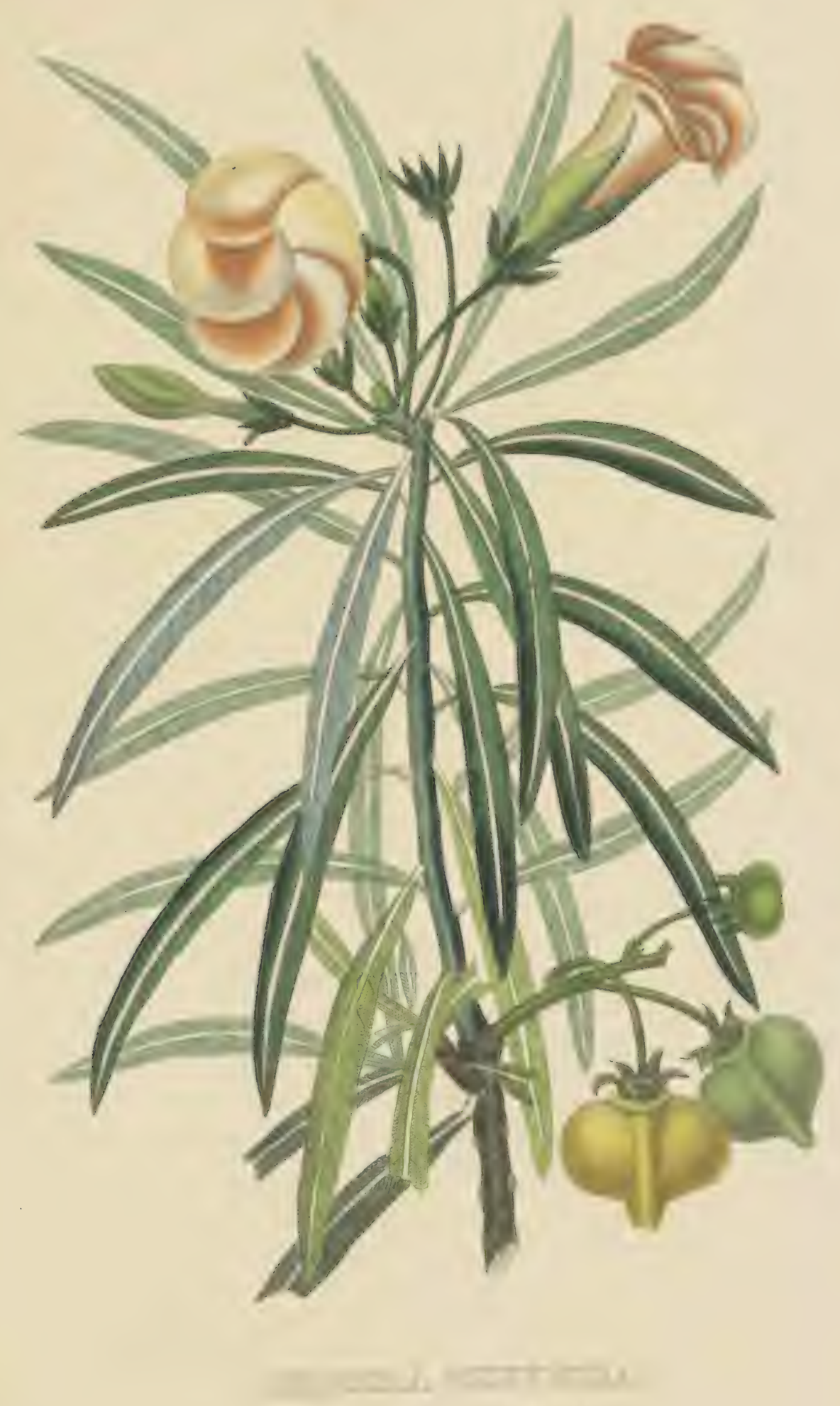

ATOUAT DHS ANTIIBS 


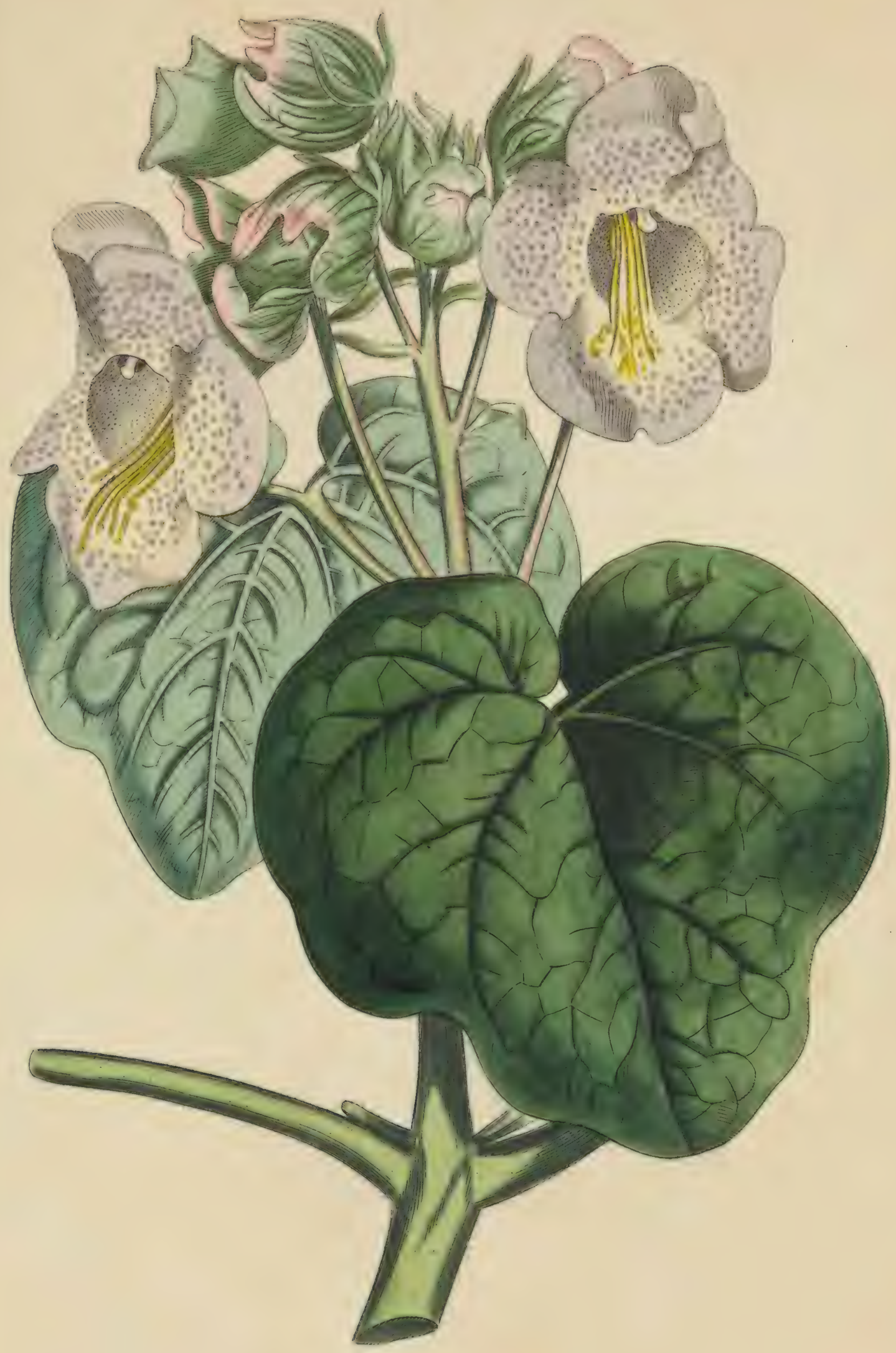

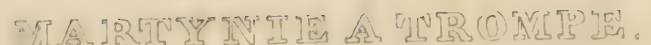

MARTYNIA PROBOSCTDEA 


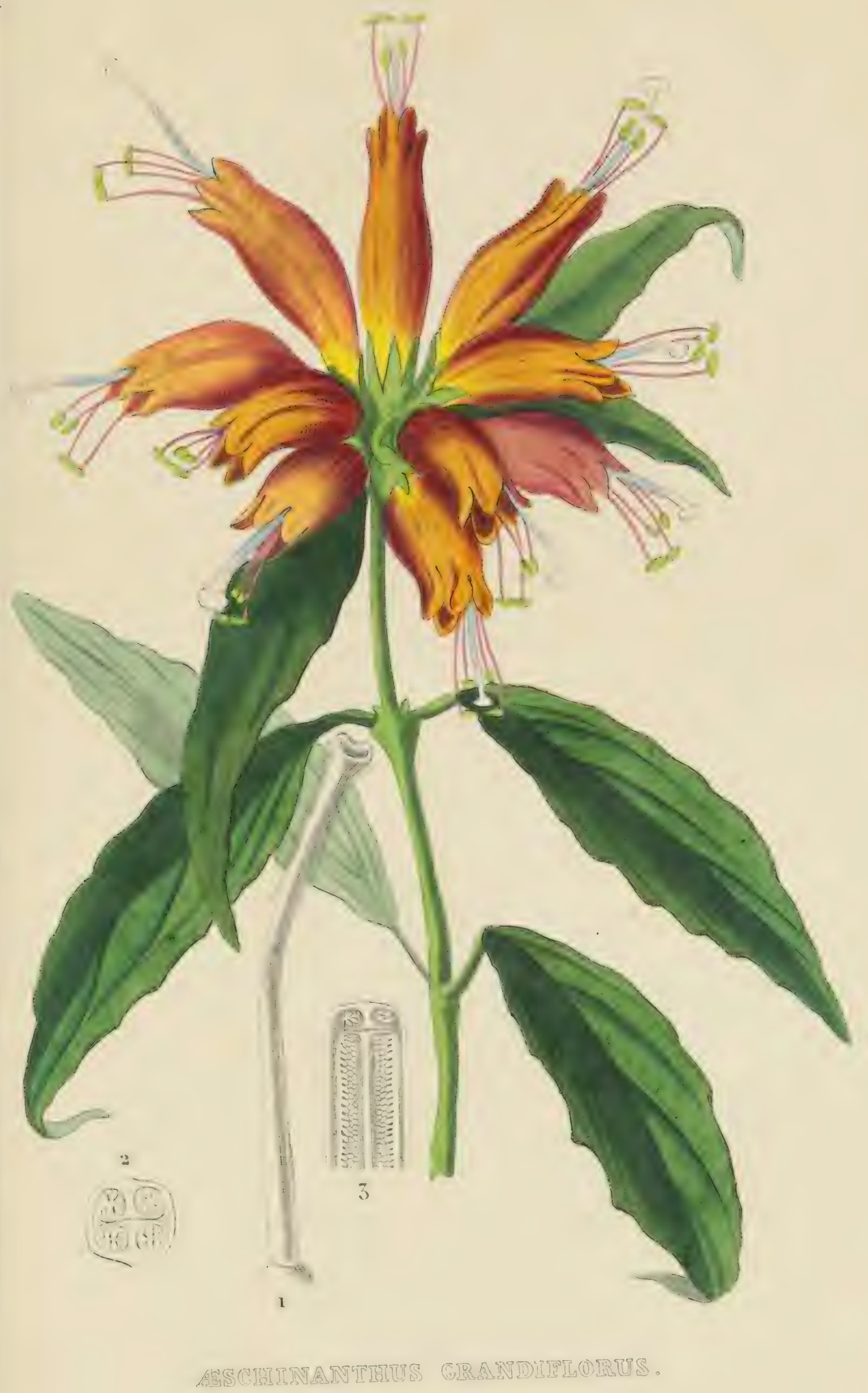

RSCHINANTHE A GRANDES ELEURS

1. L'ovaire 2. Le même coupé transversalement. 3. Le mème coupé longitudinalement. 


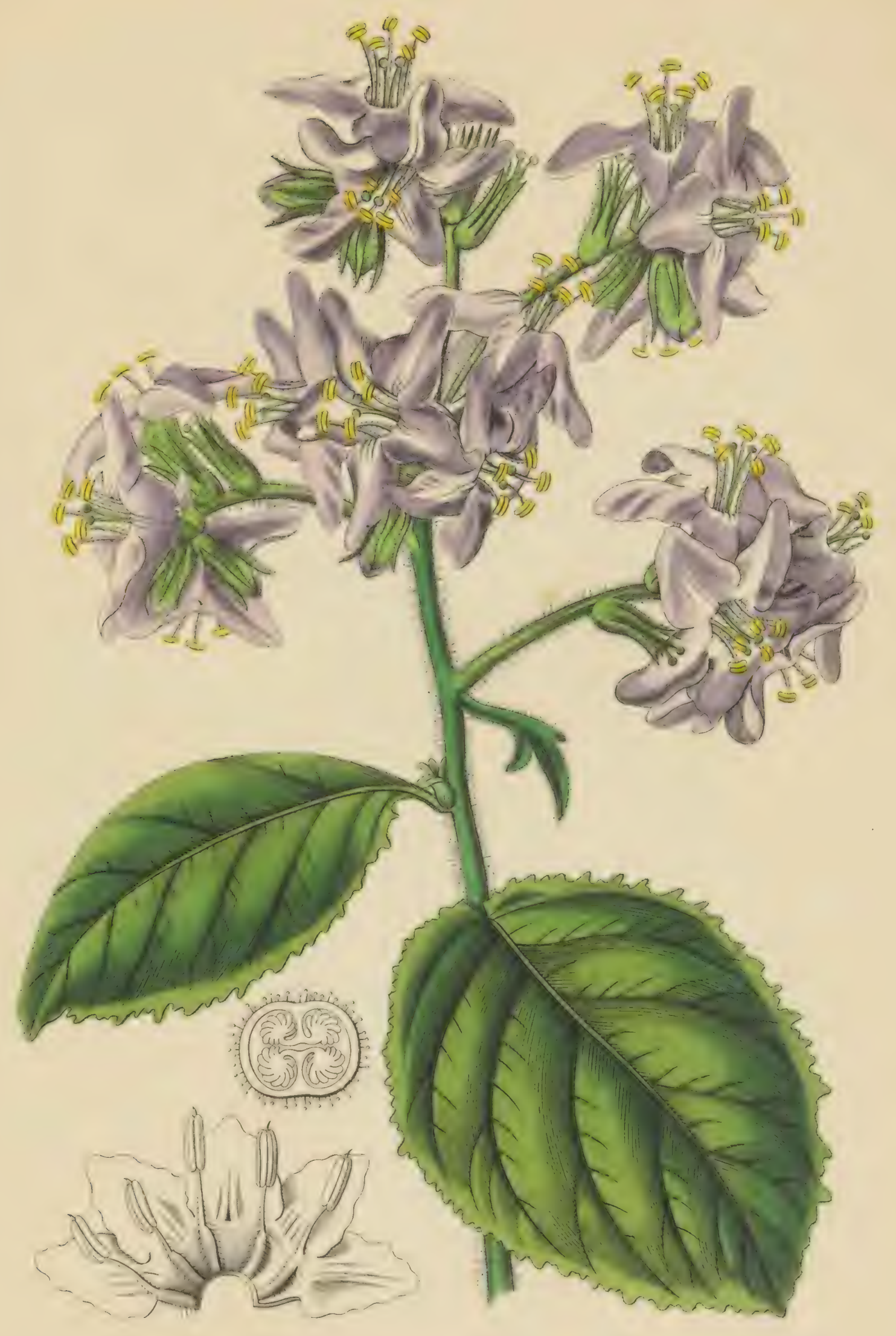

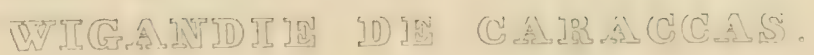

IVIGANDIA CARACCASANA 


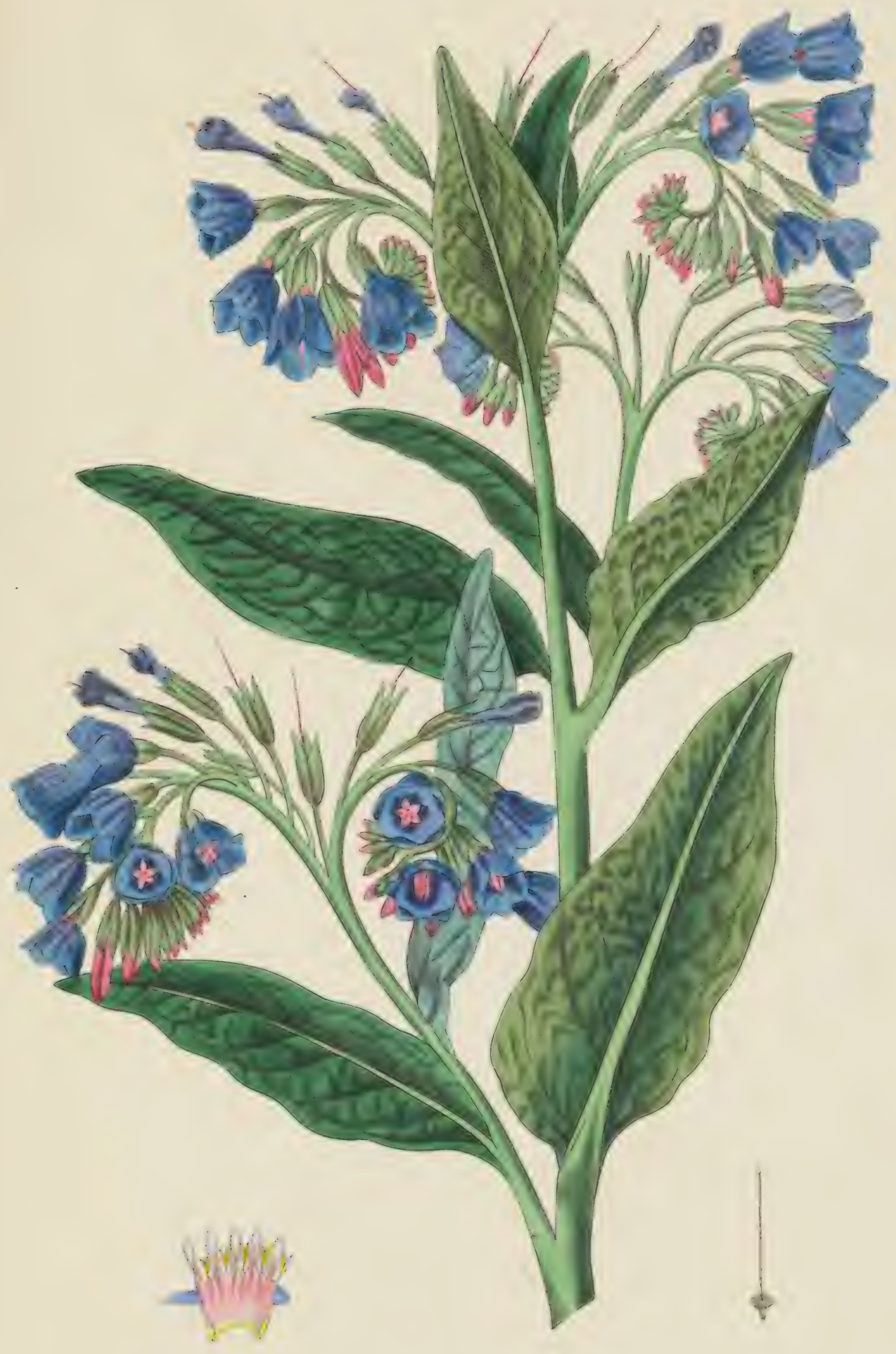

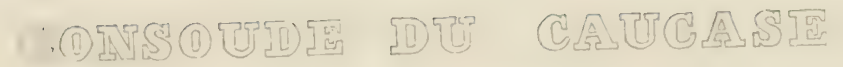

SYMPHYTUM CAUCASICUM 


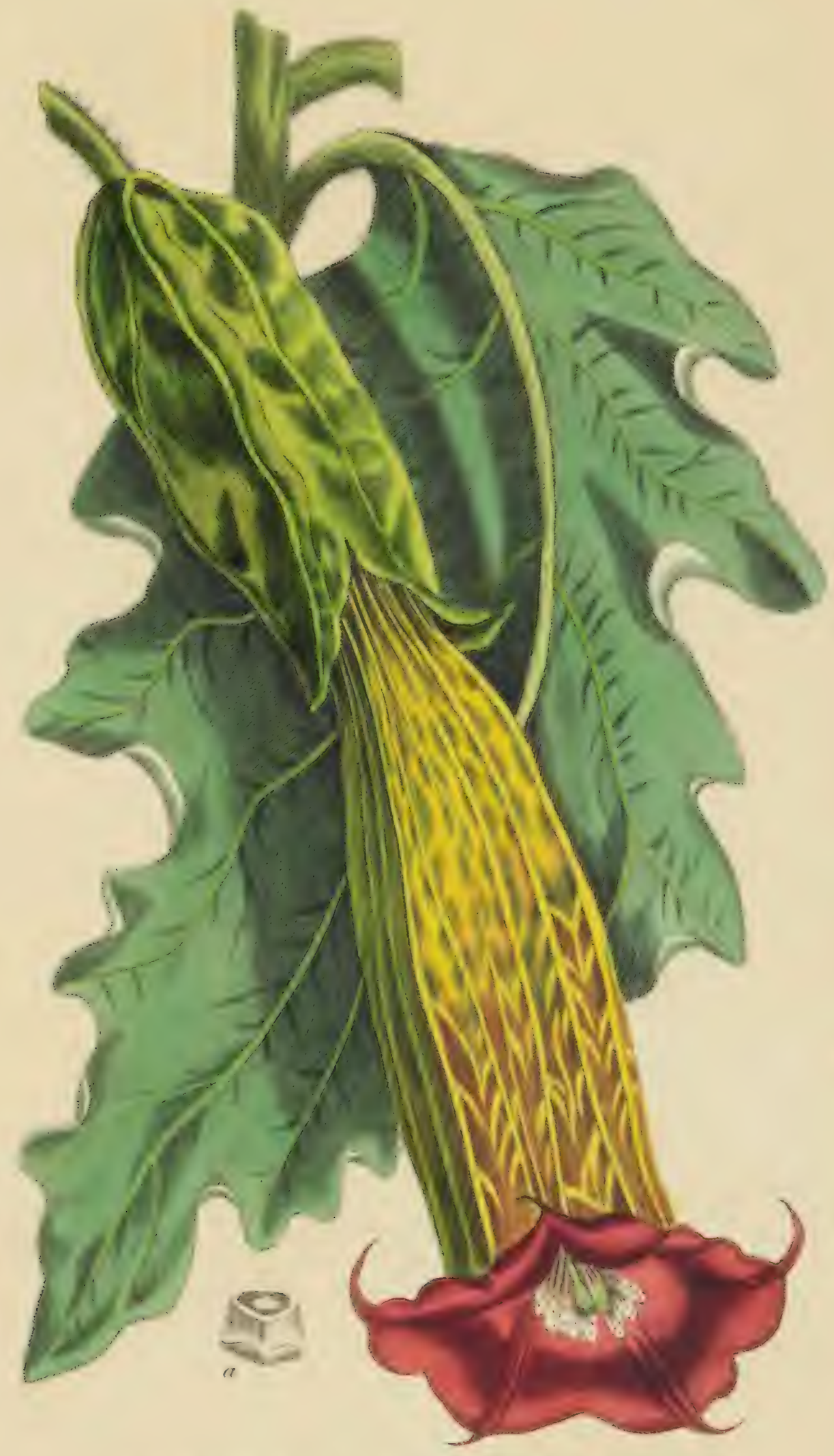

TBIRTY (GMA ANSIA BICOUOIR.

BRUGMANSIT A DEUX COULEURS. 


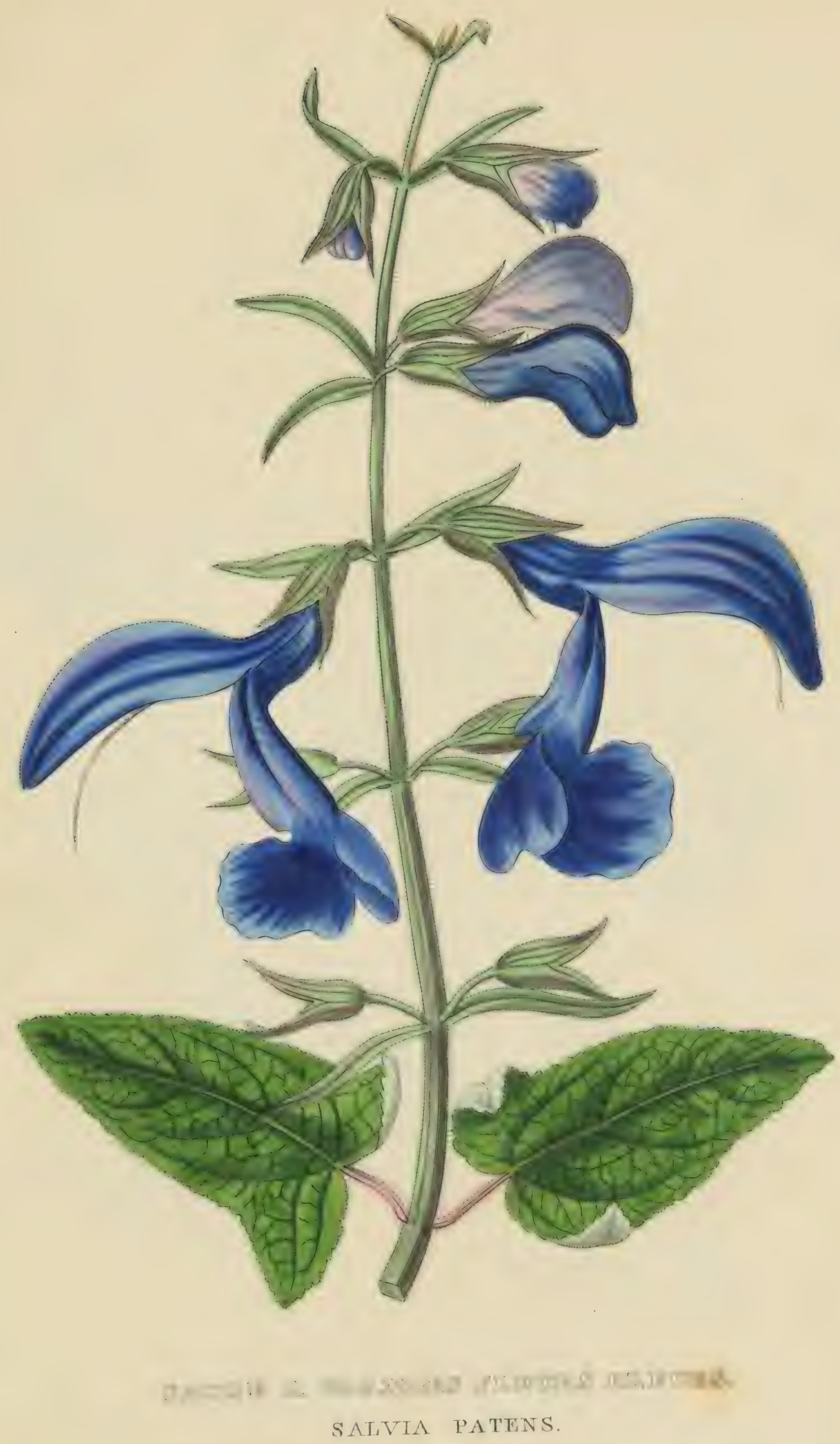




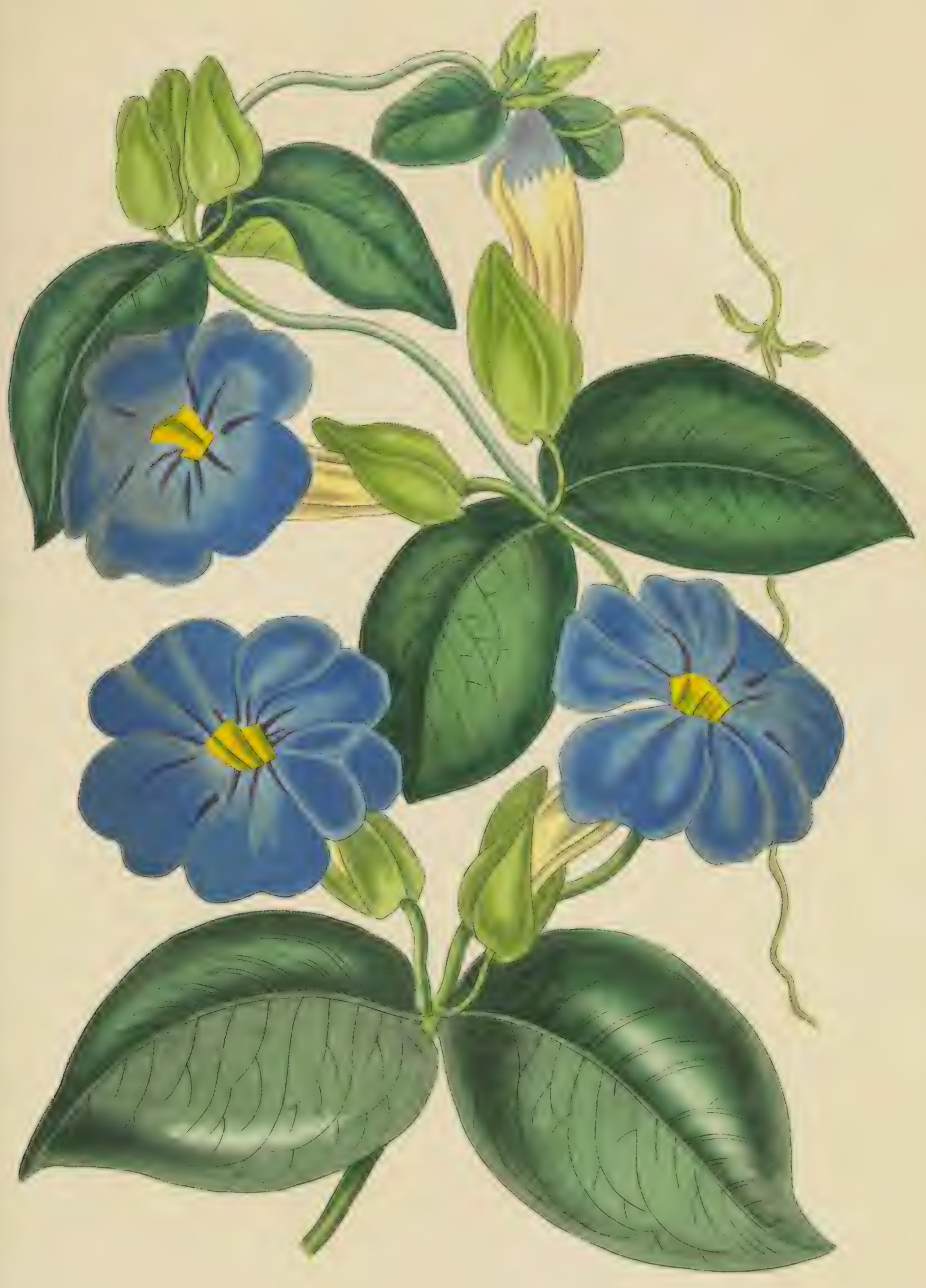

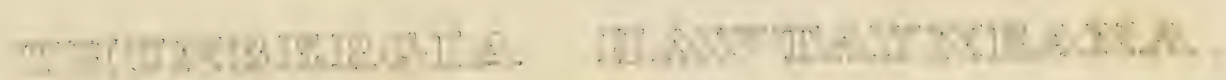
THUNBERGIE DE HAWTAYNE. 

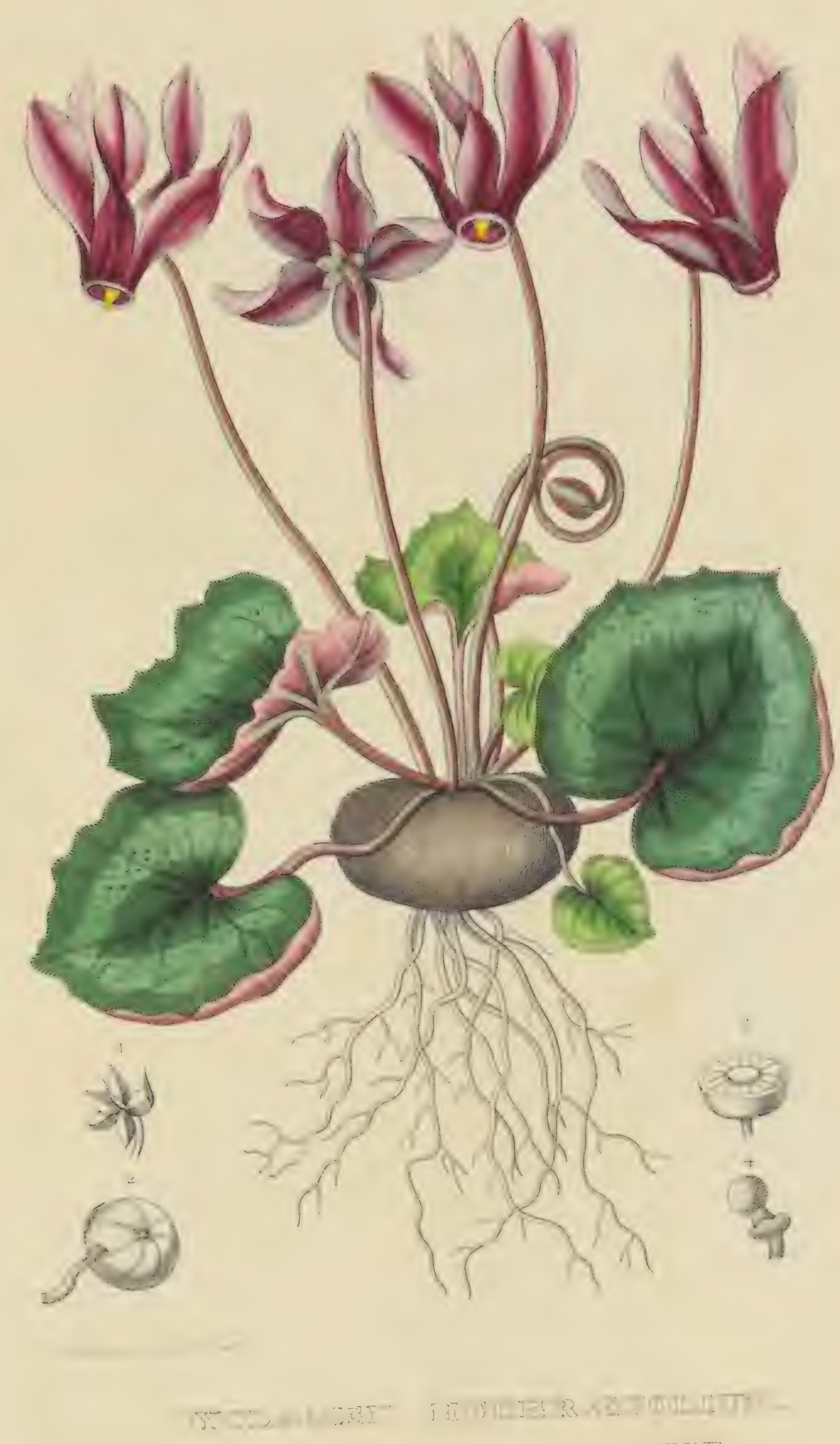

CYCIAANE A FEUILIES DTI IIERRF 

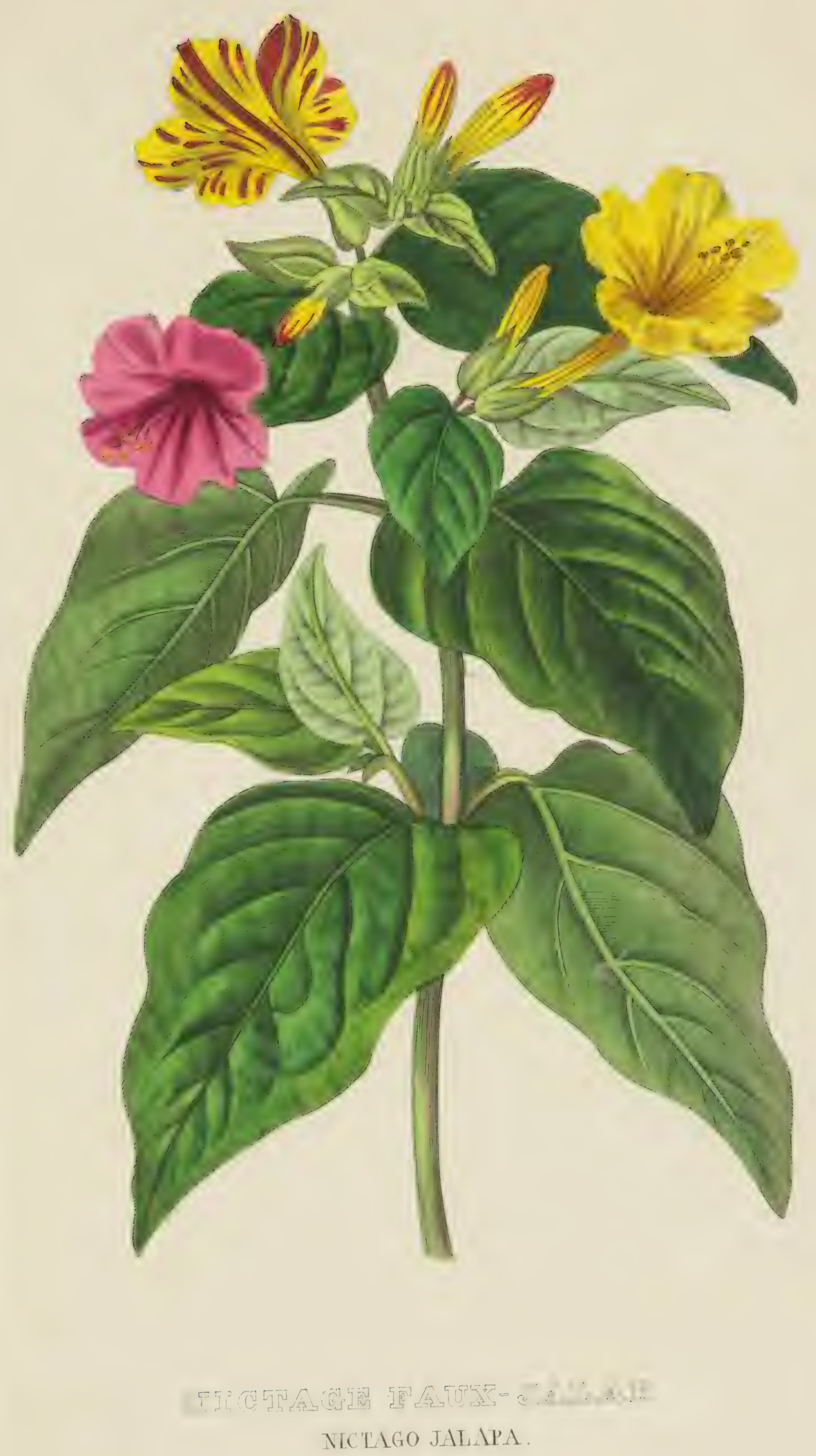

NICLAGO JALAPA. 


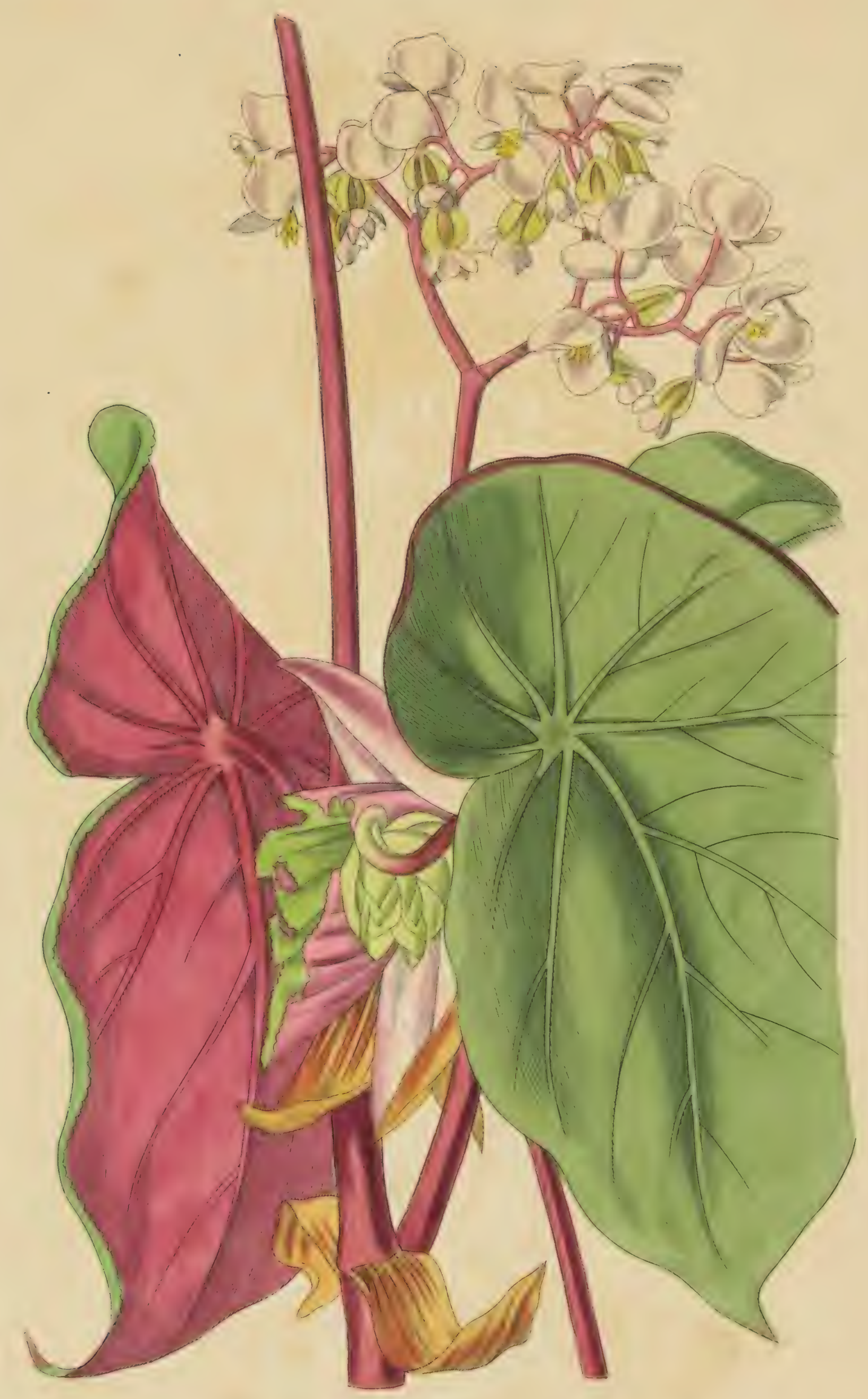

Mrot

BBEGONIA SANGUTNEA. 


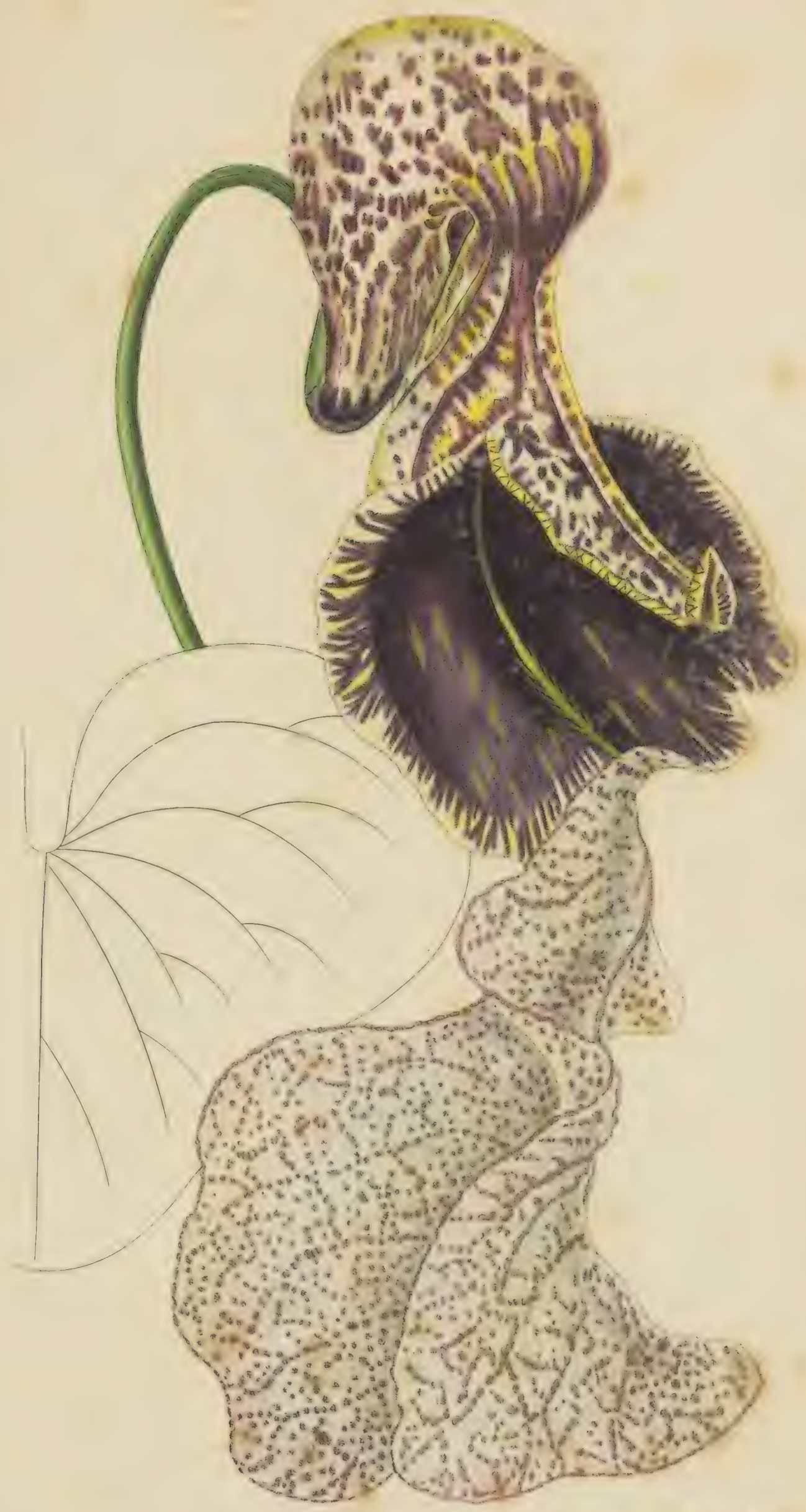

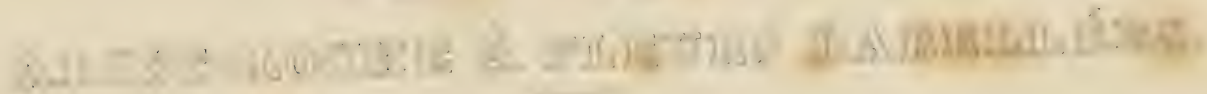




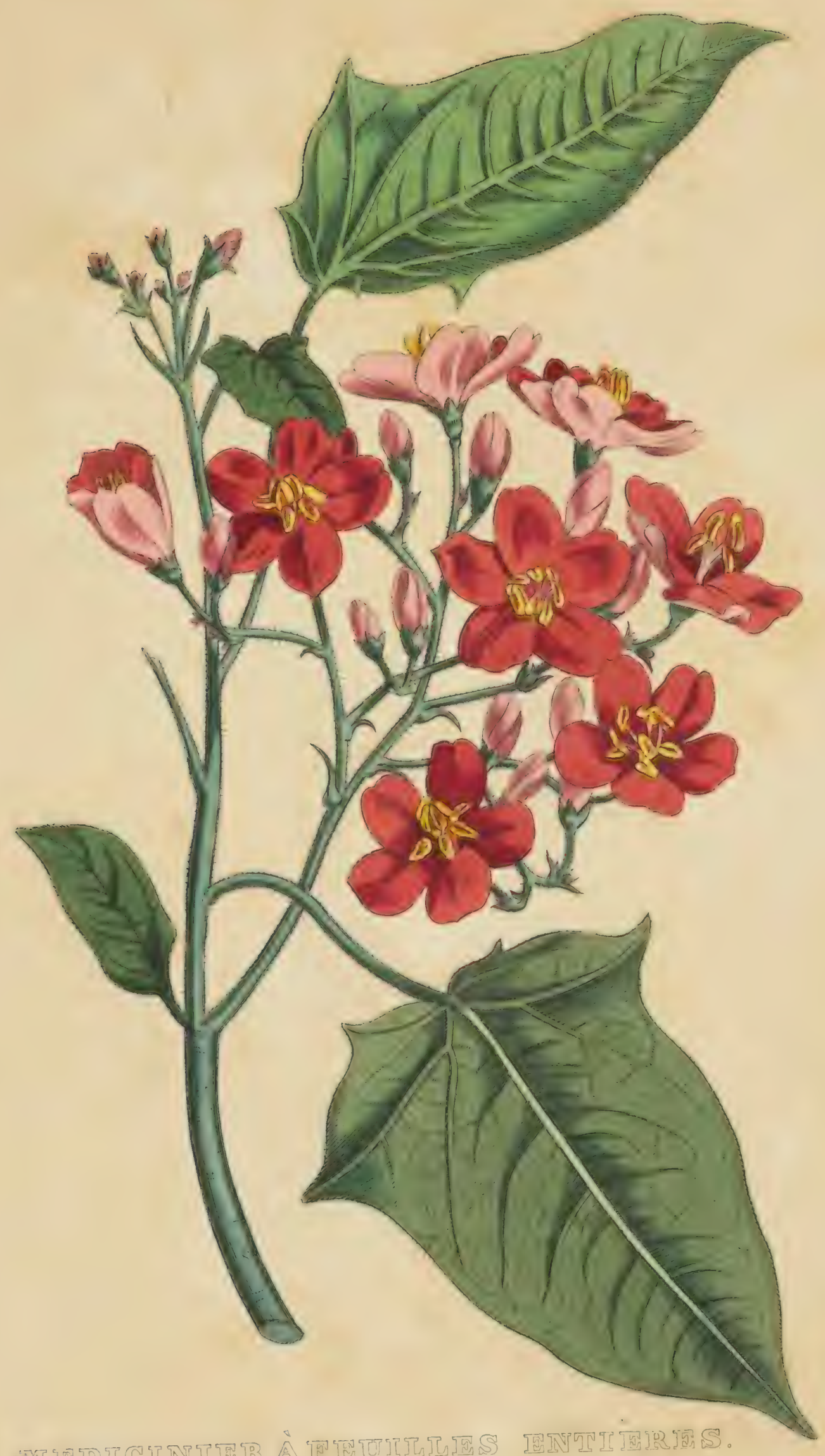

JATROPHA INTHGERRIMA 


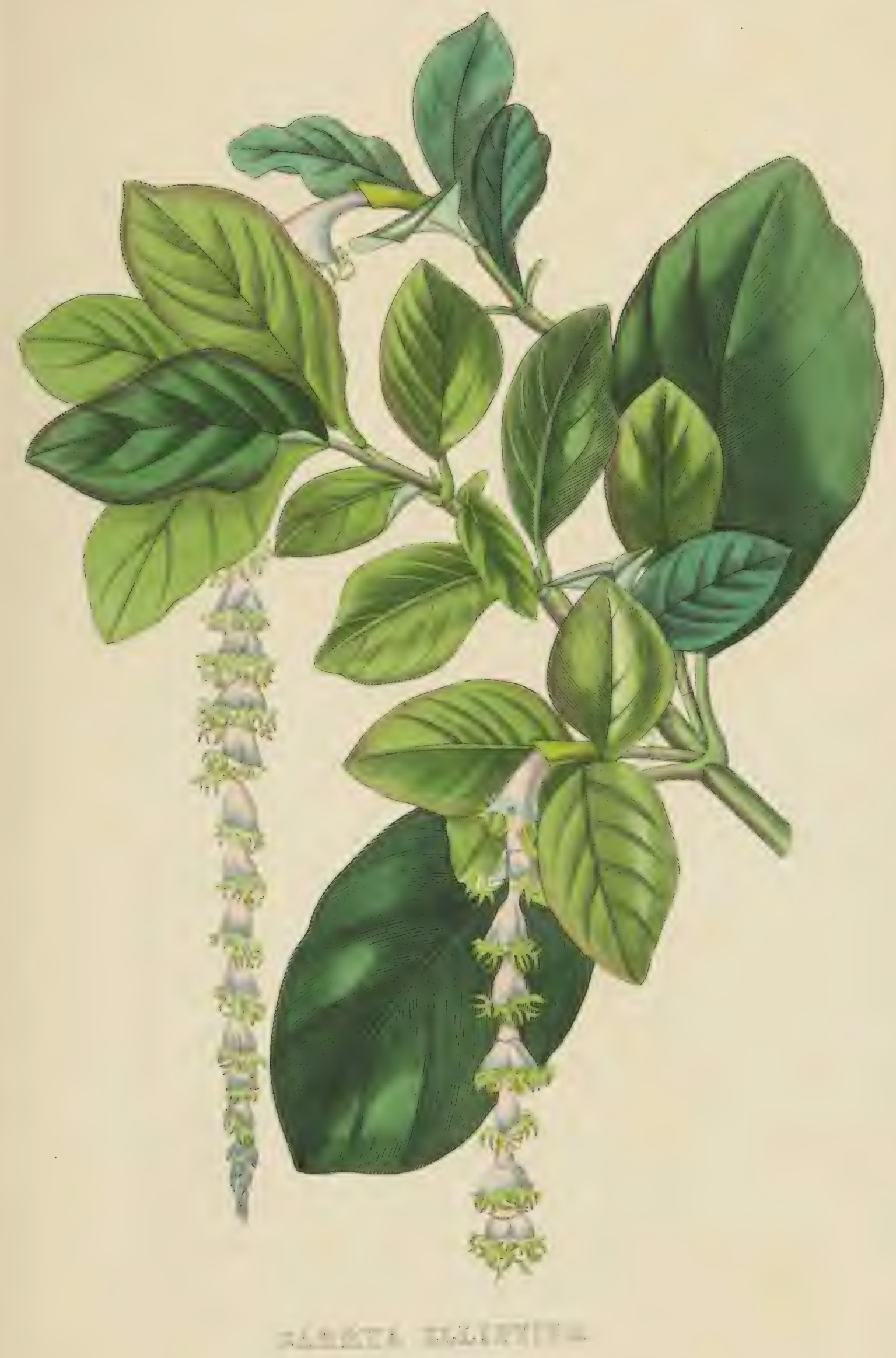

GARRYE A FEUILLES ELLIPTIQUES . 


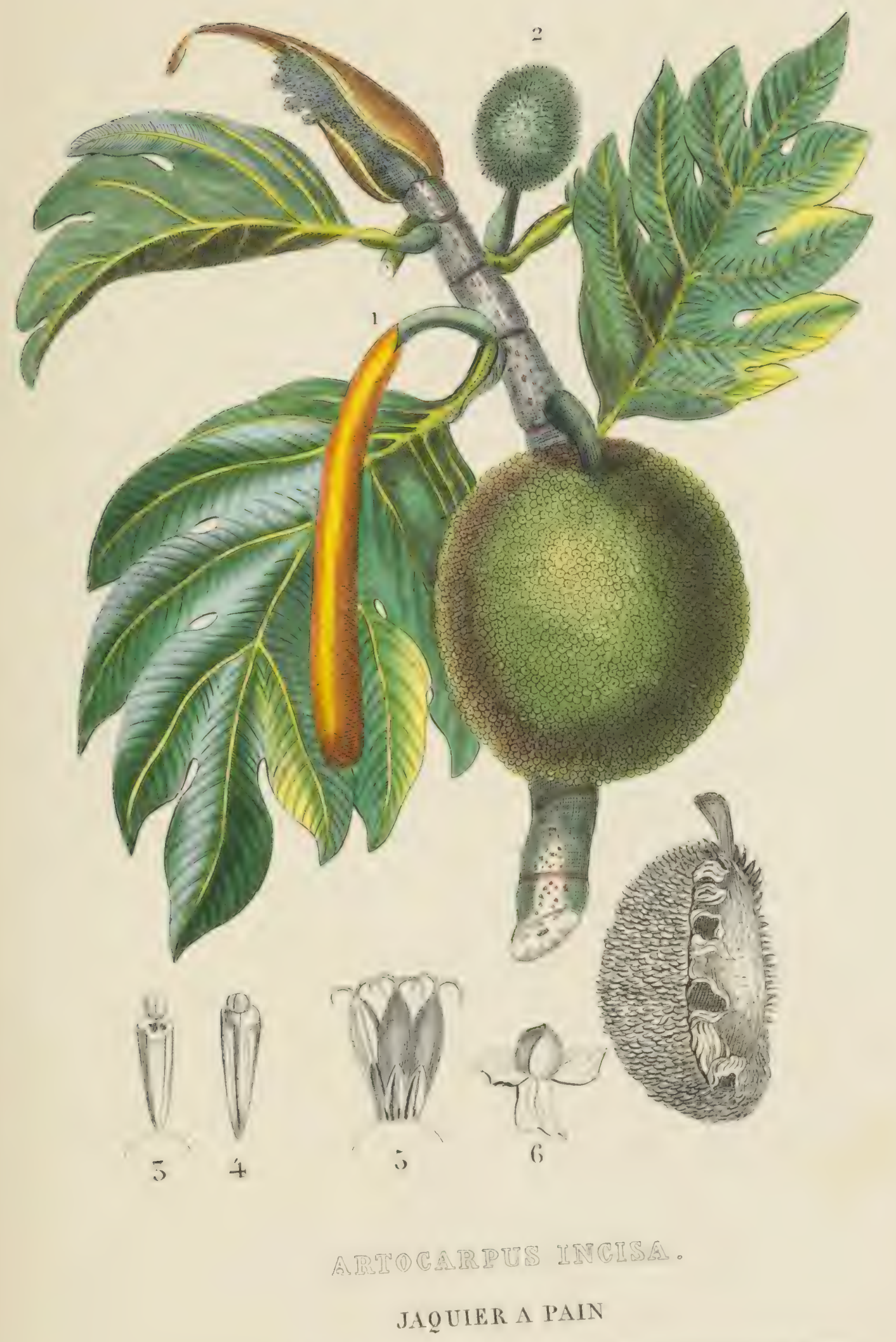

Chaton mâte . 2.id femelle 3 et 4 . Fleurs mâles. 5. Fleurs femelles . 6. Graine. 


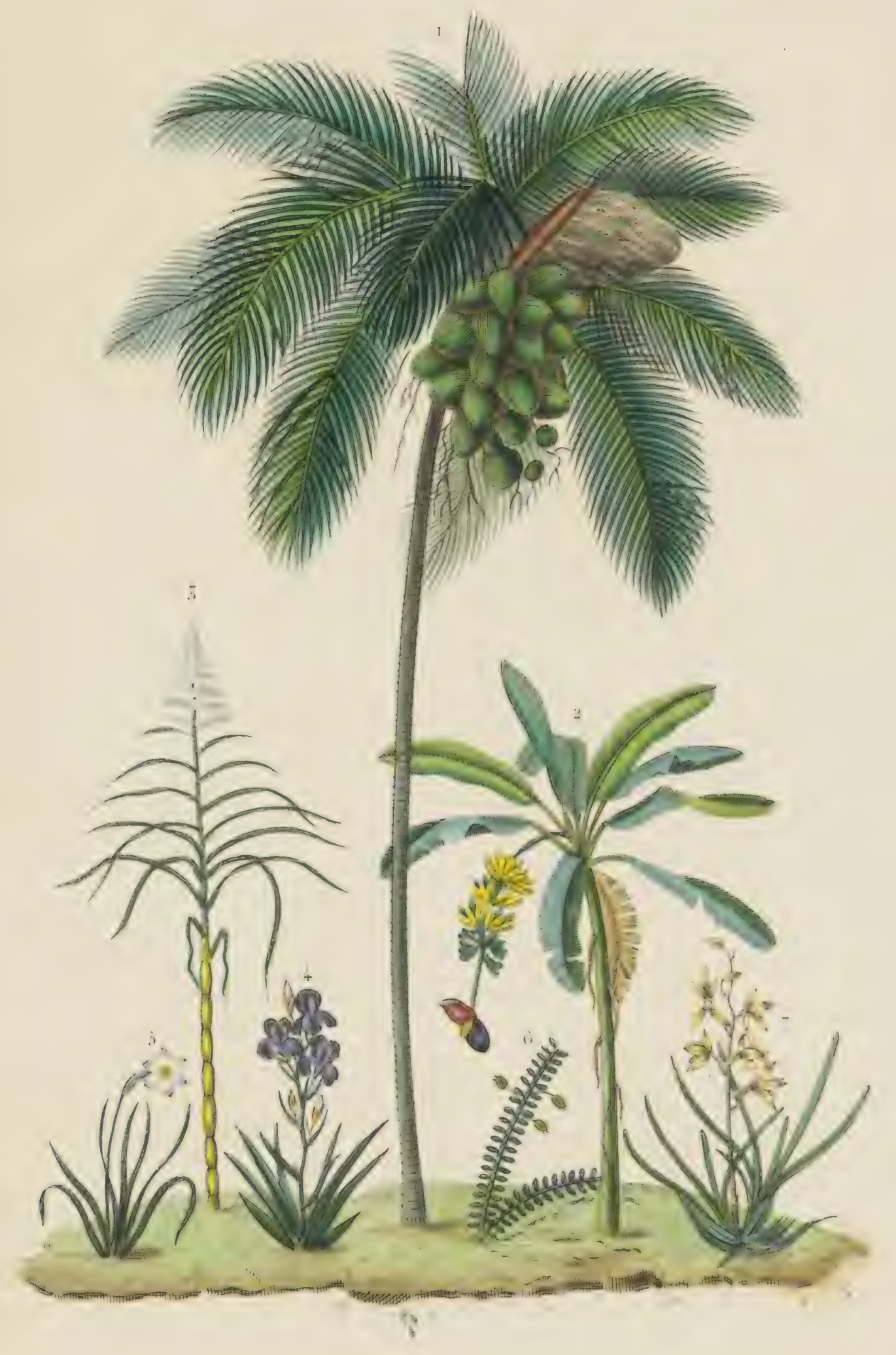

\footnotetext{
1. COCO nucifera.

2. MUSA paradisiaca.

+. IRIS èrmanica

- SACCHARUM officinarum. 6. CYIIBUIUIII cchinocarpon.

7. FiPII)FNDRUM sinense.
} 


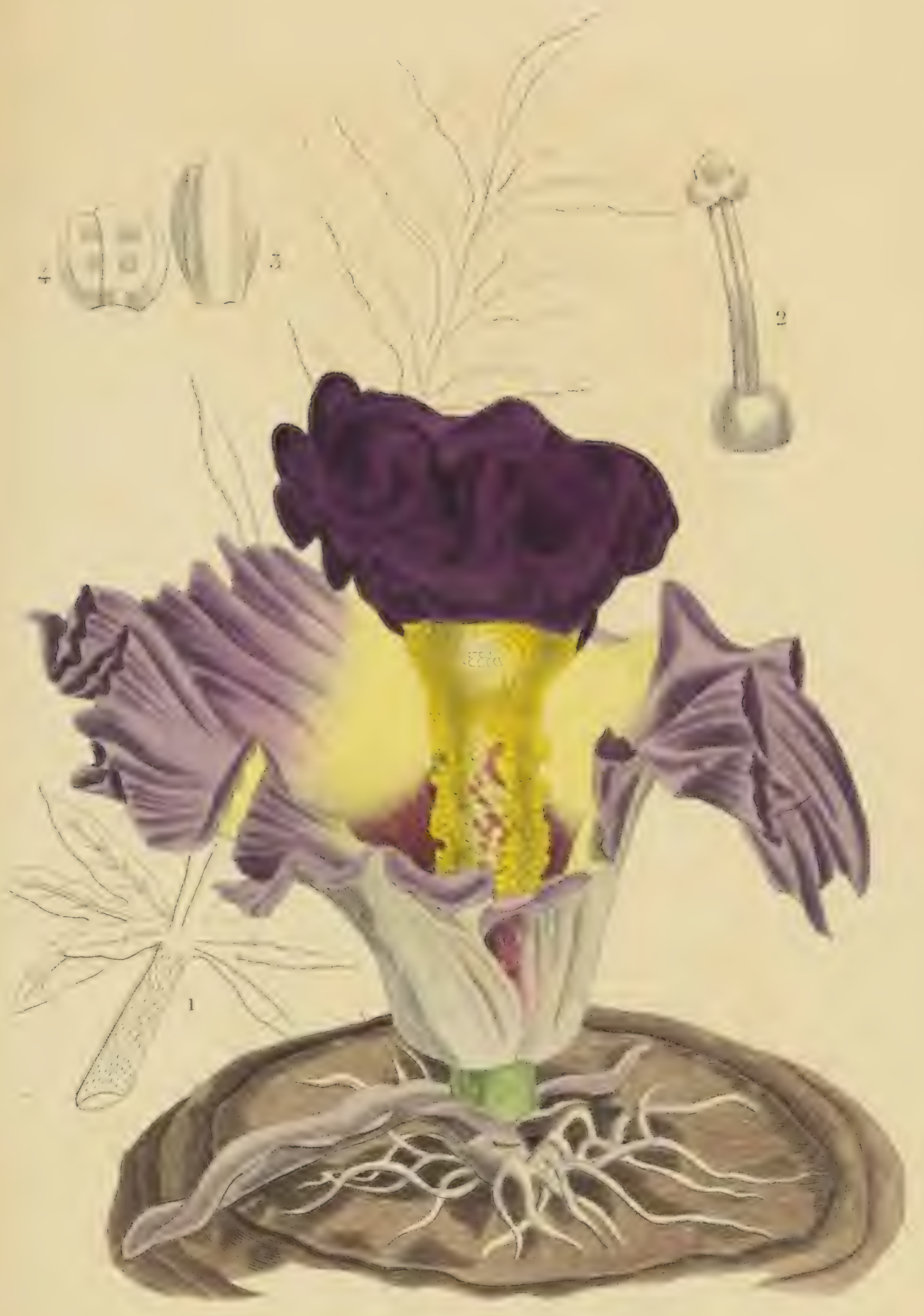

AITUM CGAIPANUIATUMI

GOUNT CAMPANULE. 


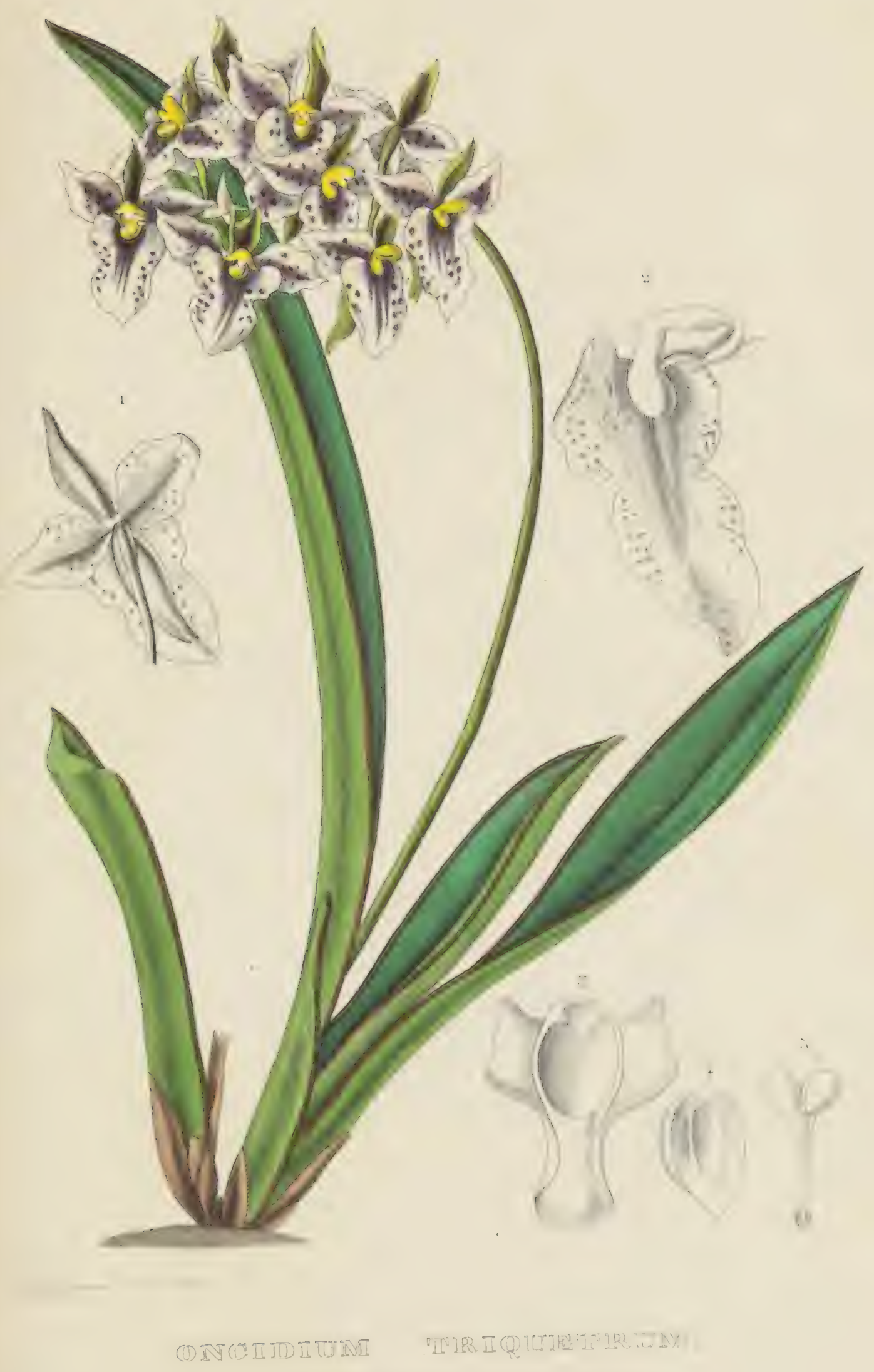

ONCIDTE A FETUILLES TRIANGULAIRHAS. 


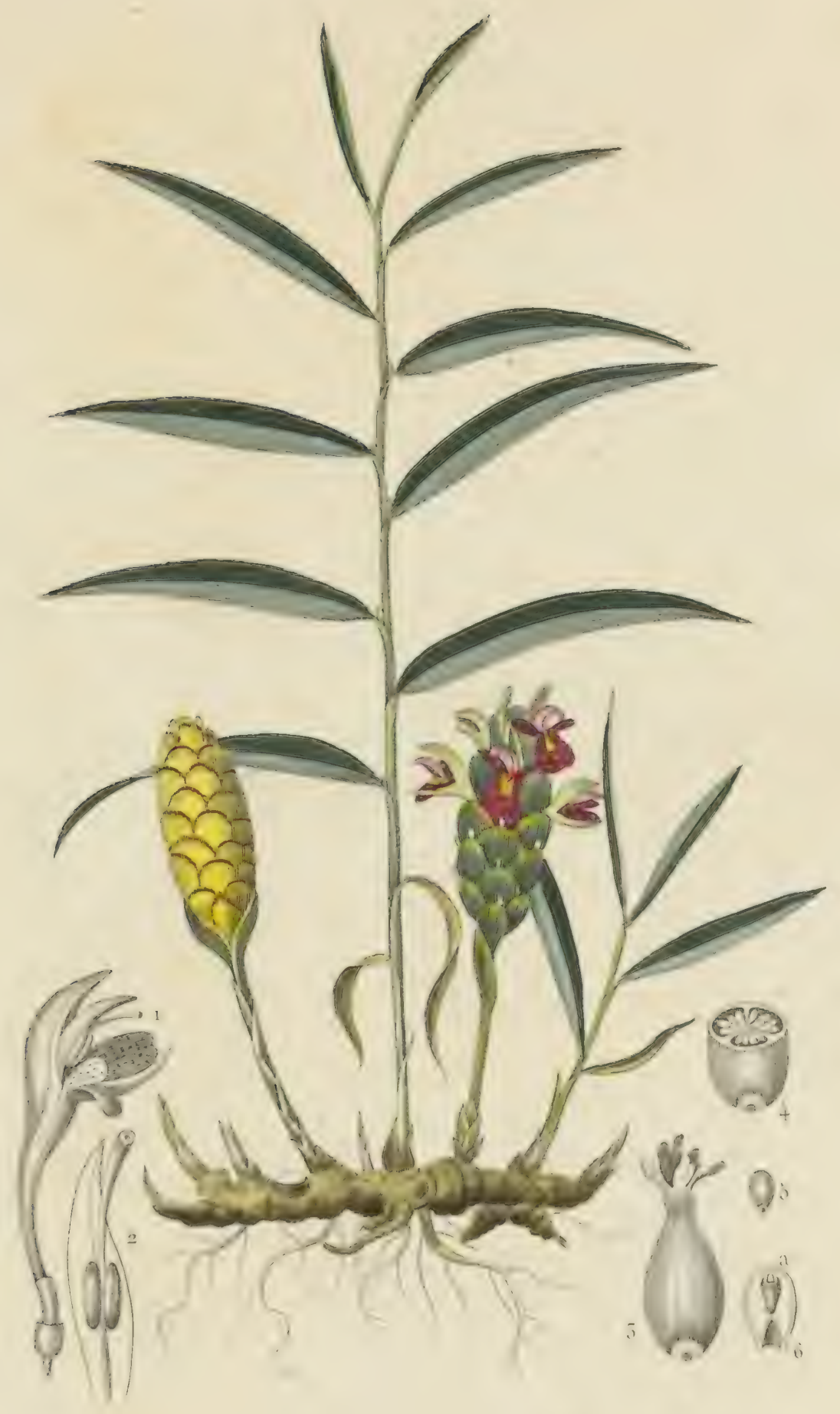

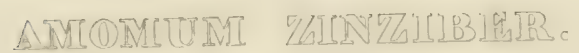

AMLNE GINGEMBRE. 


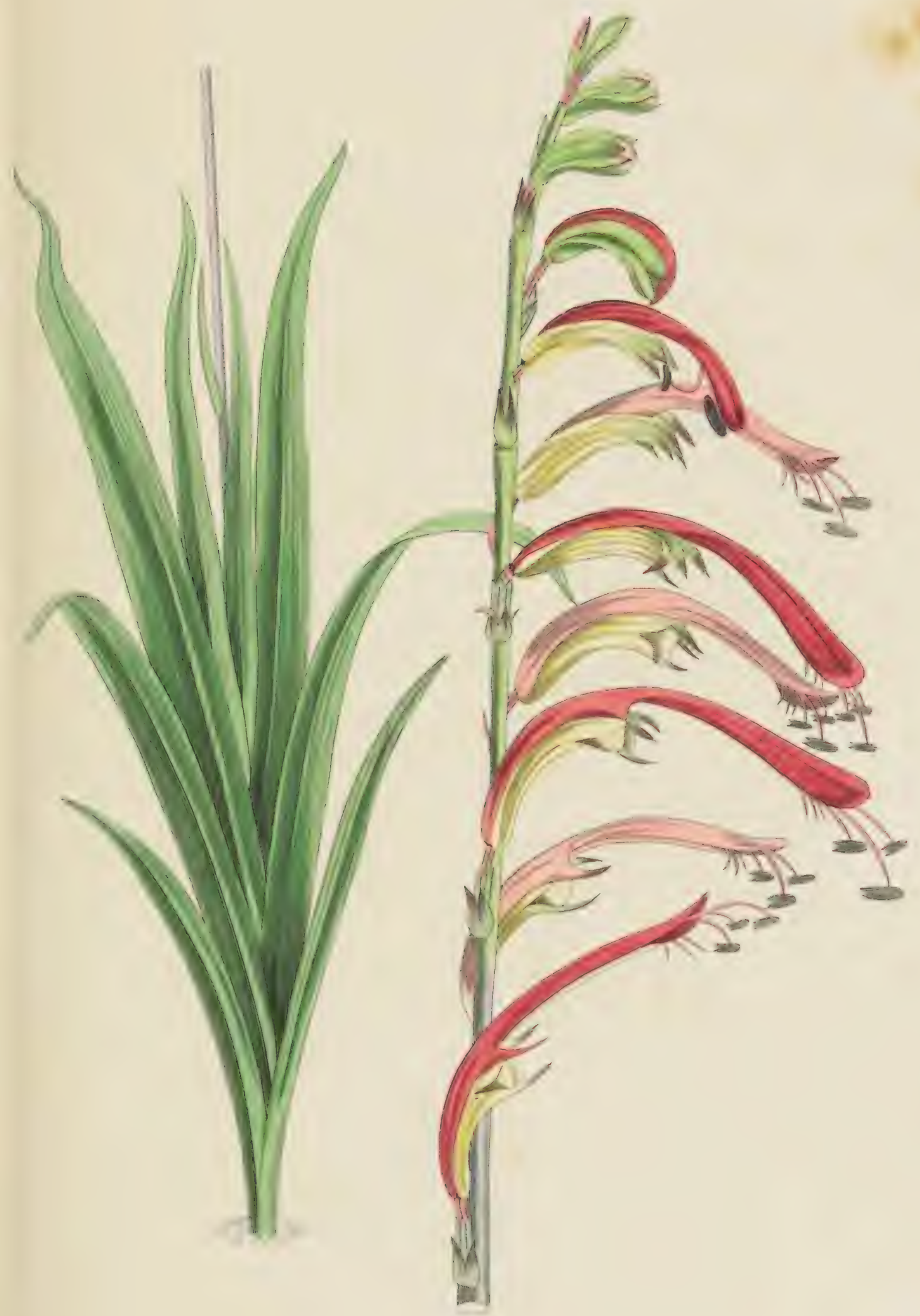

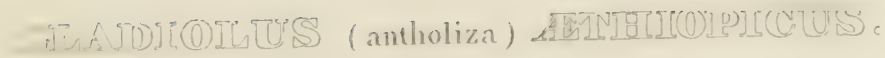

G]AYIUL T'FTHIOPIE 


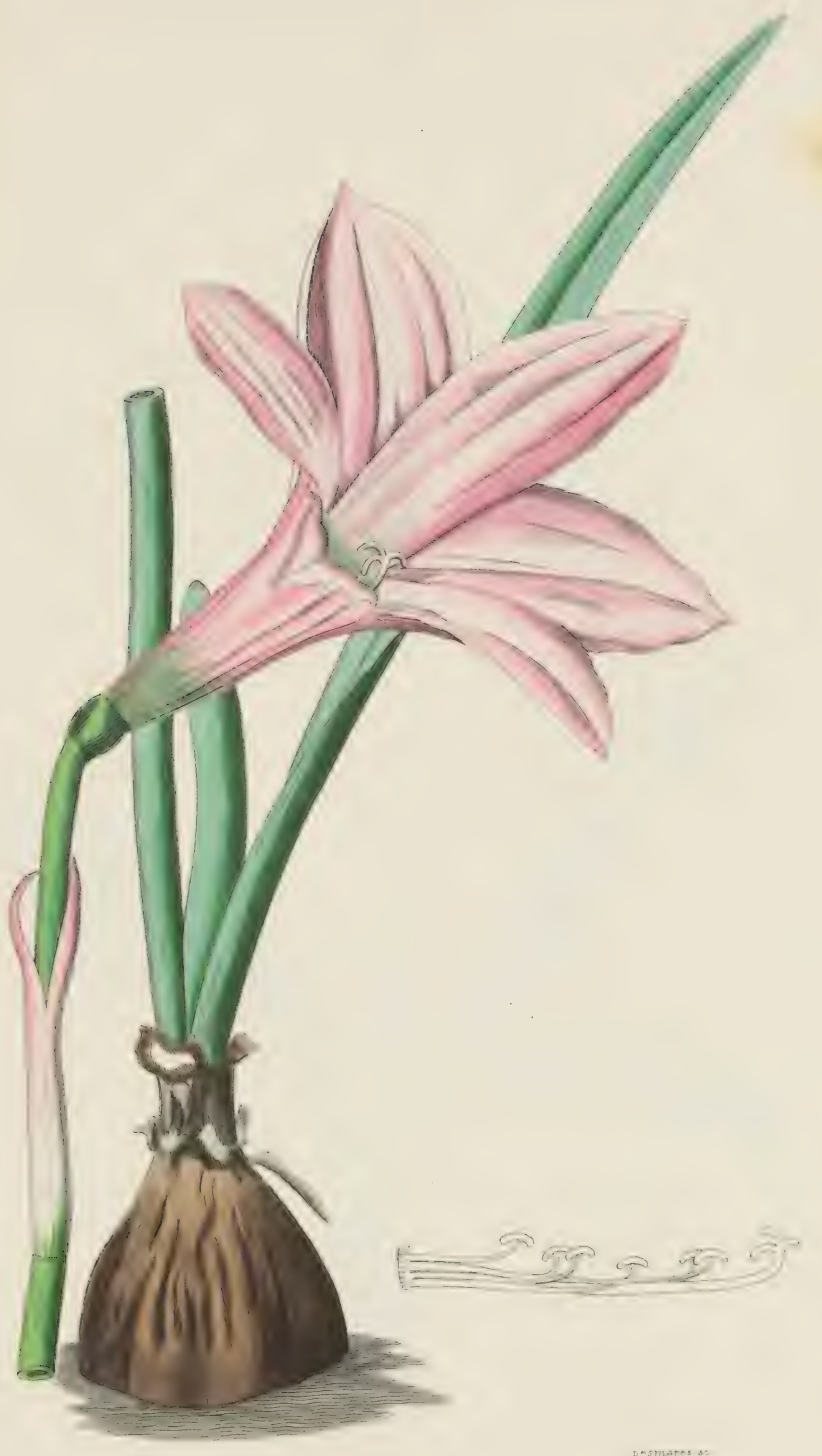

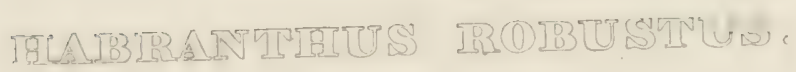
mABRANTHE ROBUSTE . 


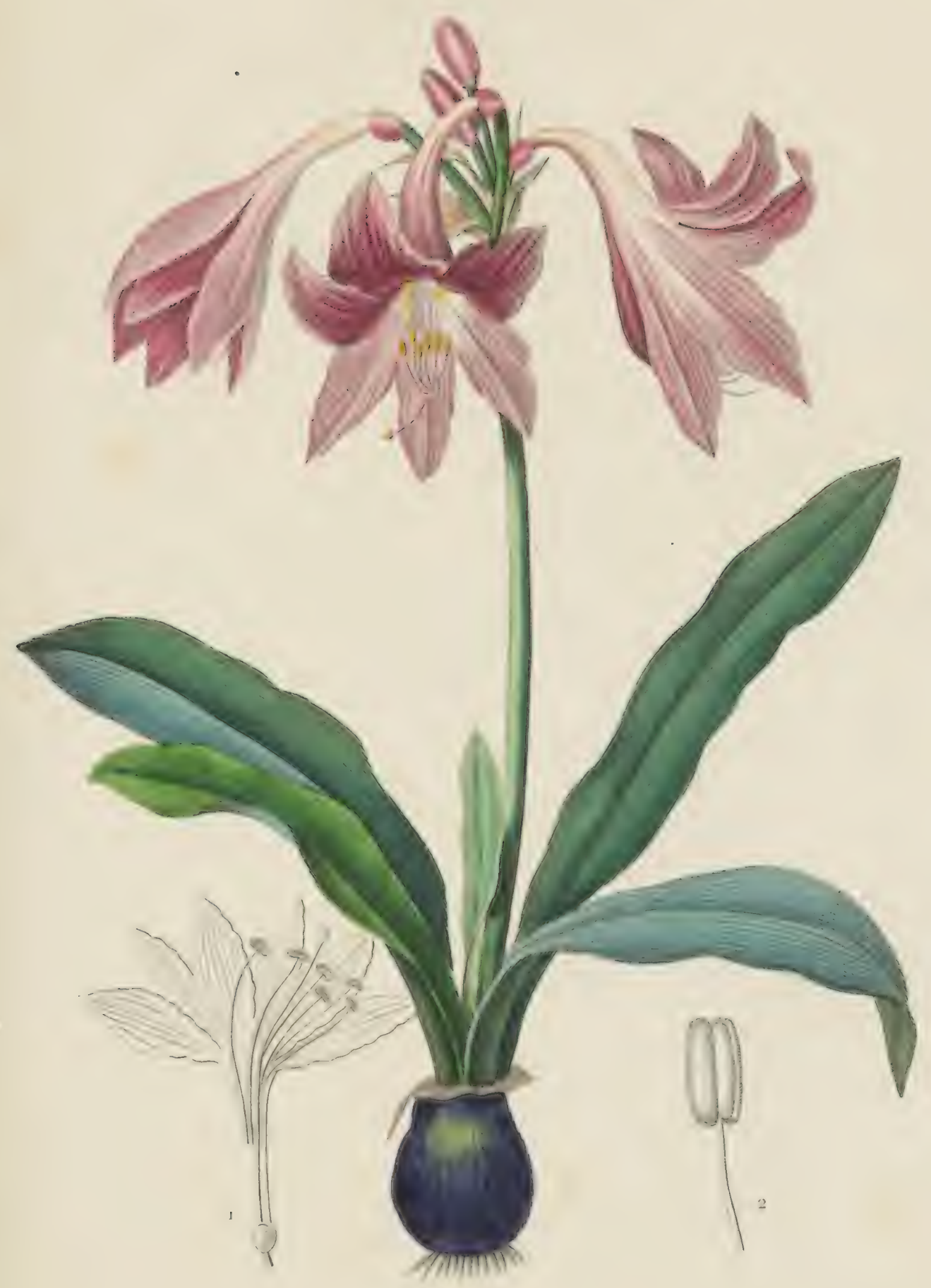

ANTRITILS RFTICULEE. 


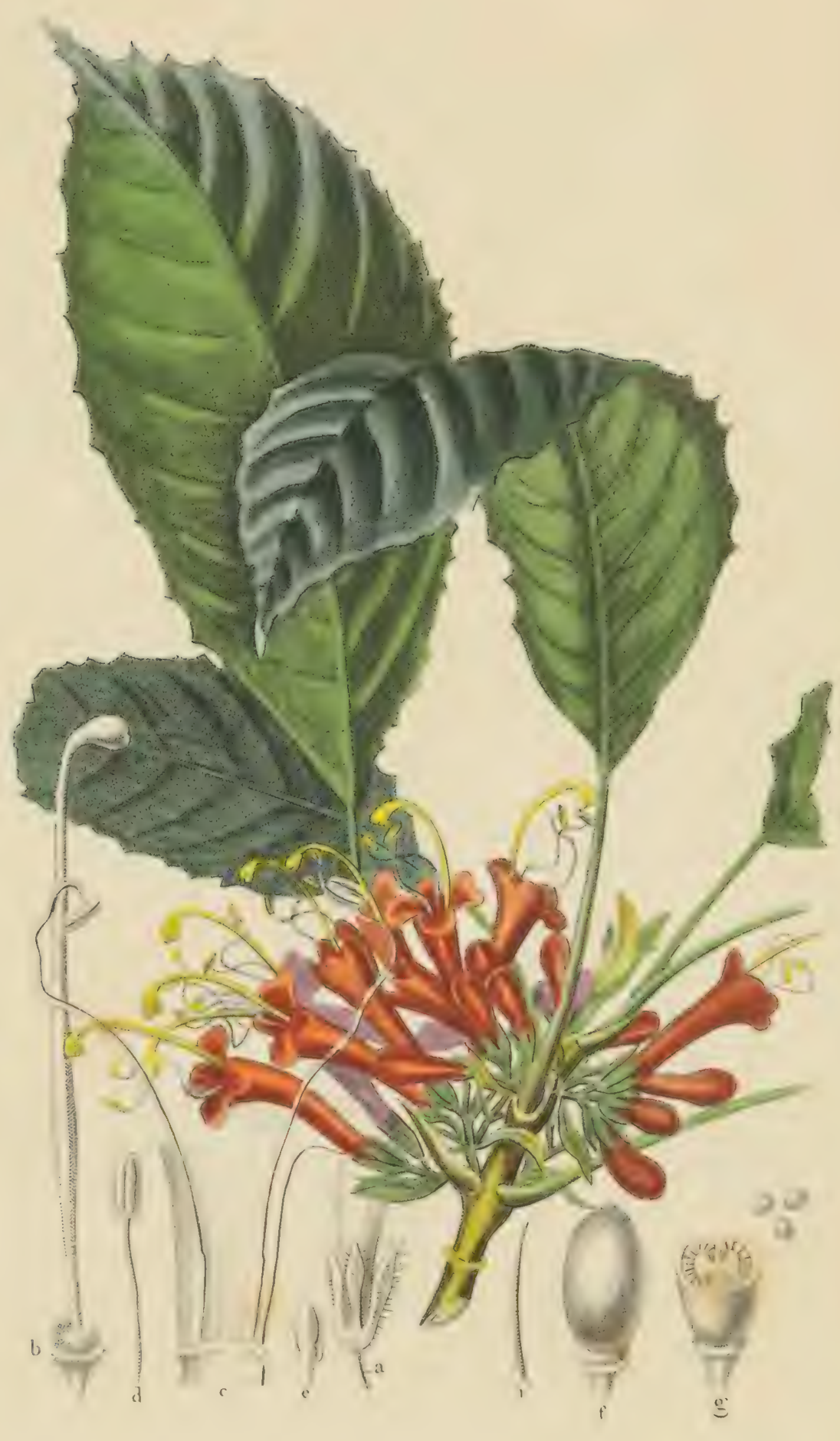

JURTTANJDIRA WTAMTITNTEA.

CYRTANDRE A LONGUES FITATINFS 


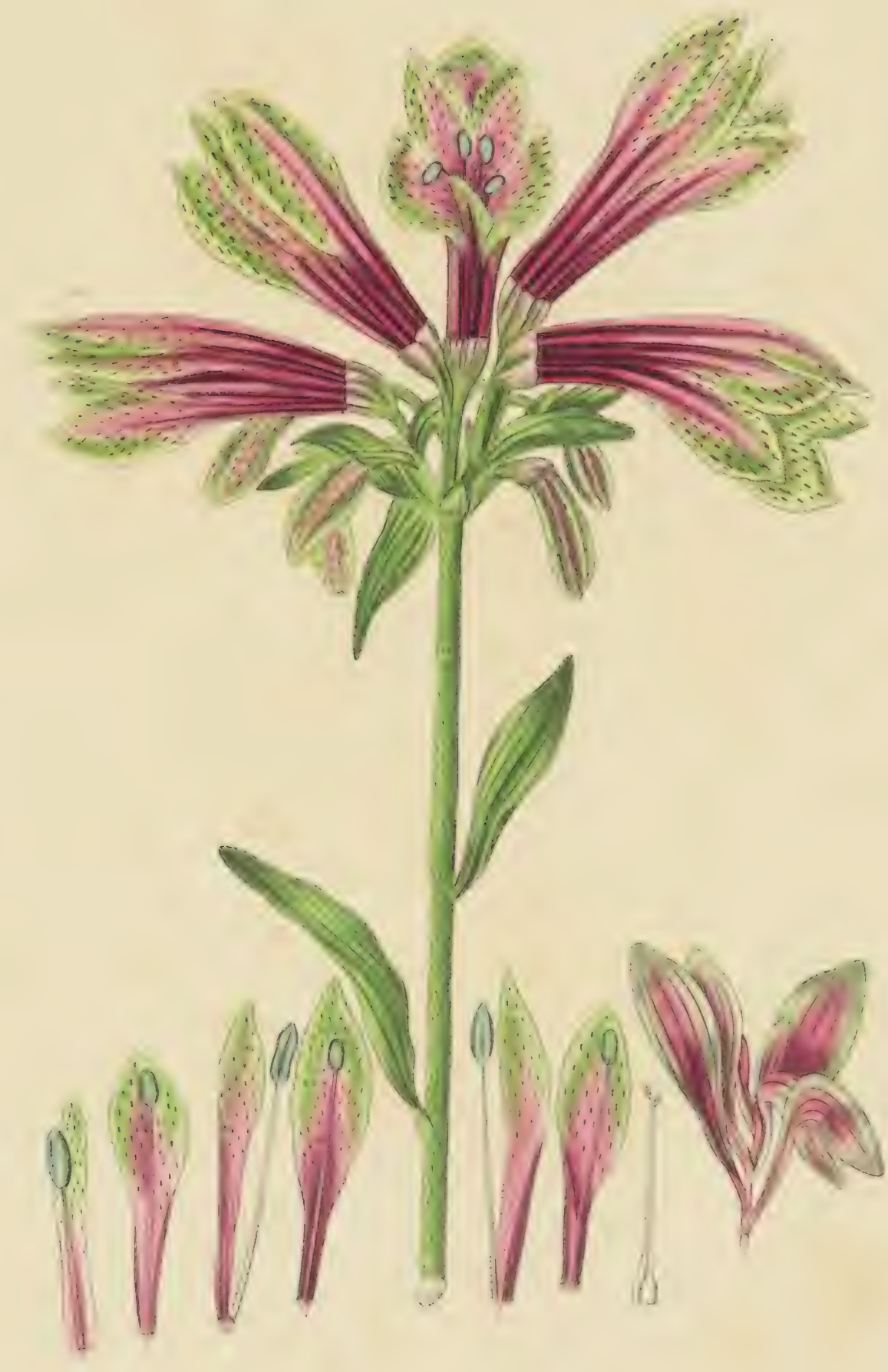

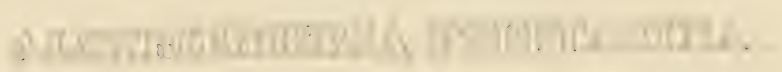

METROEMERIF PERROOUET 


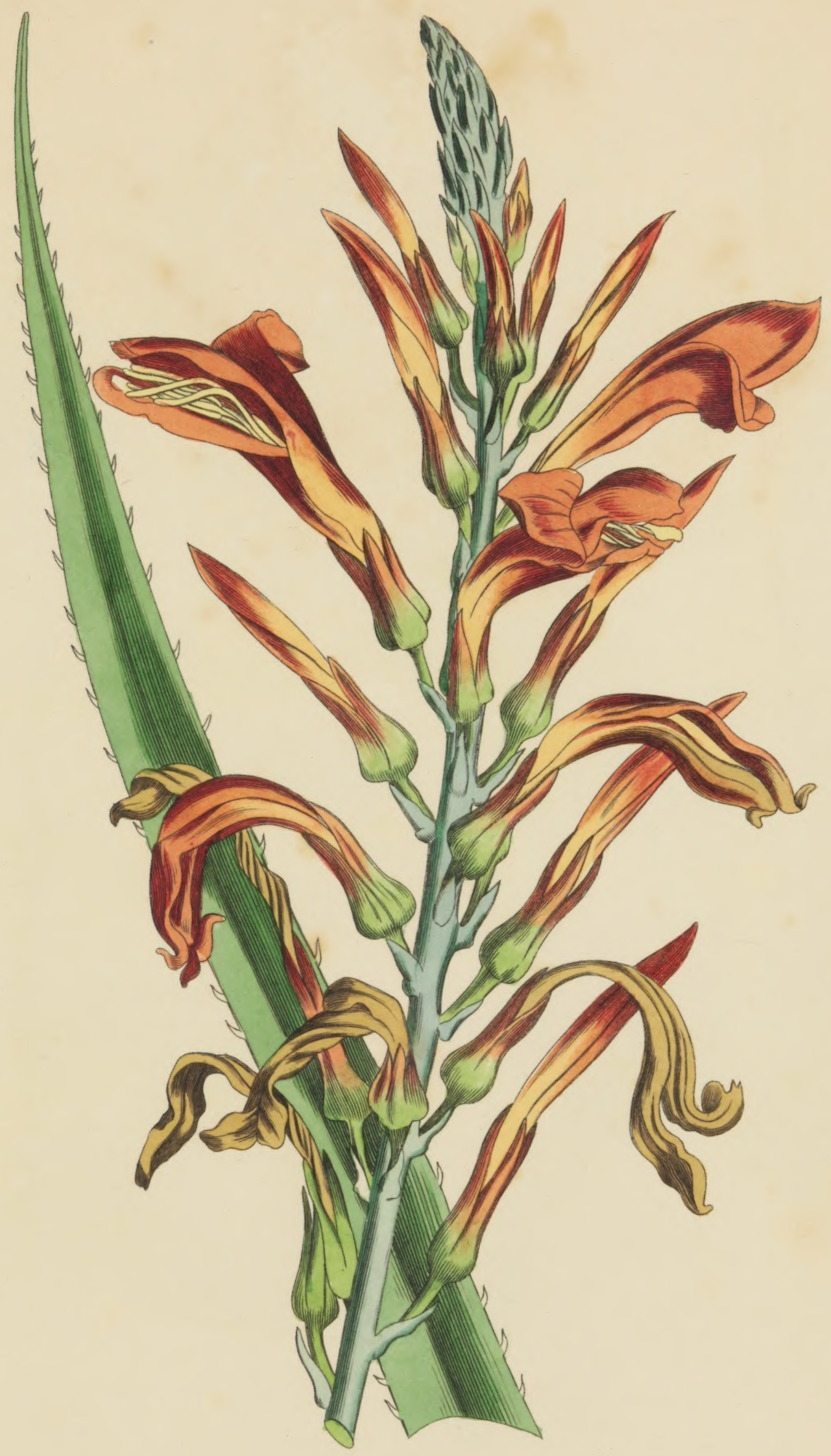

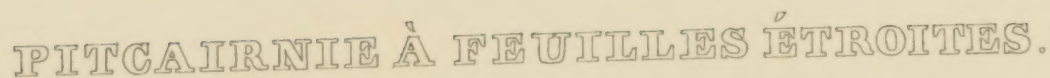

PITCAIRNIA ANGUSTIFOLIA. 


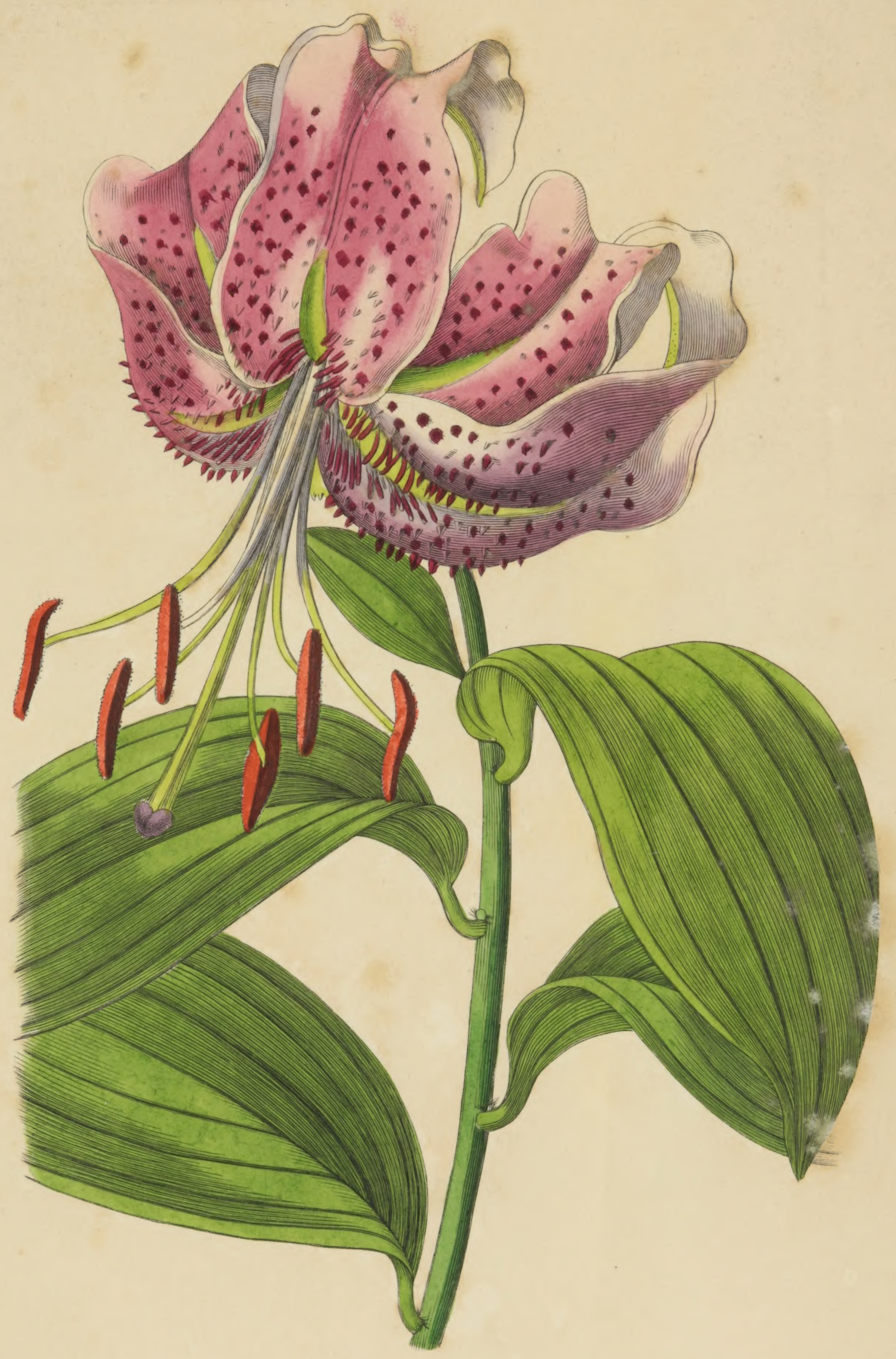

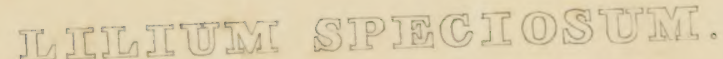

LIS ËLIGANT. 


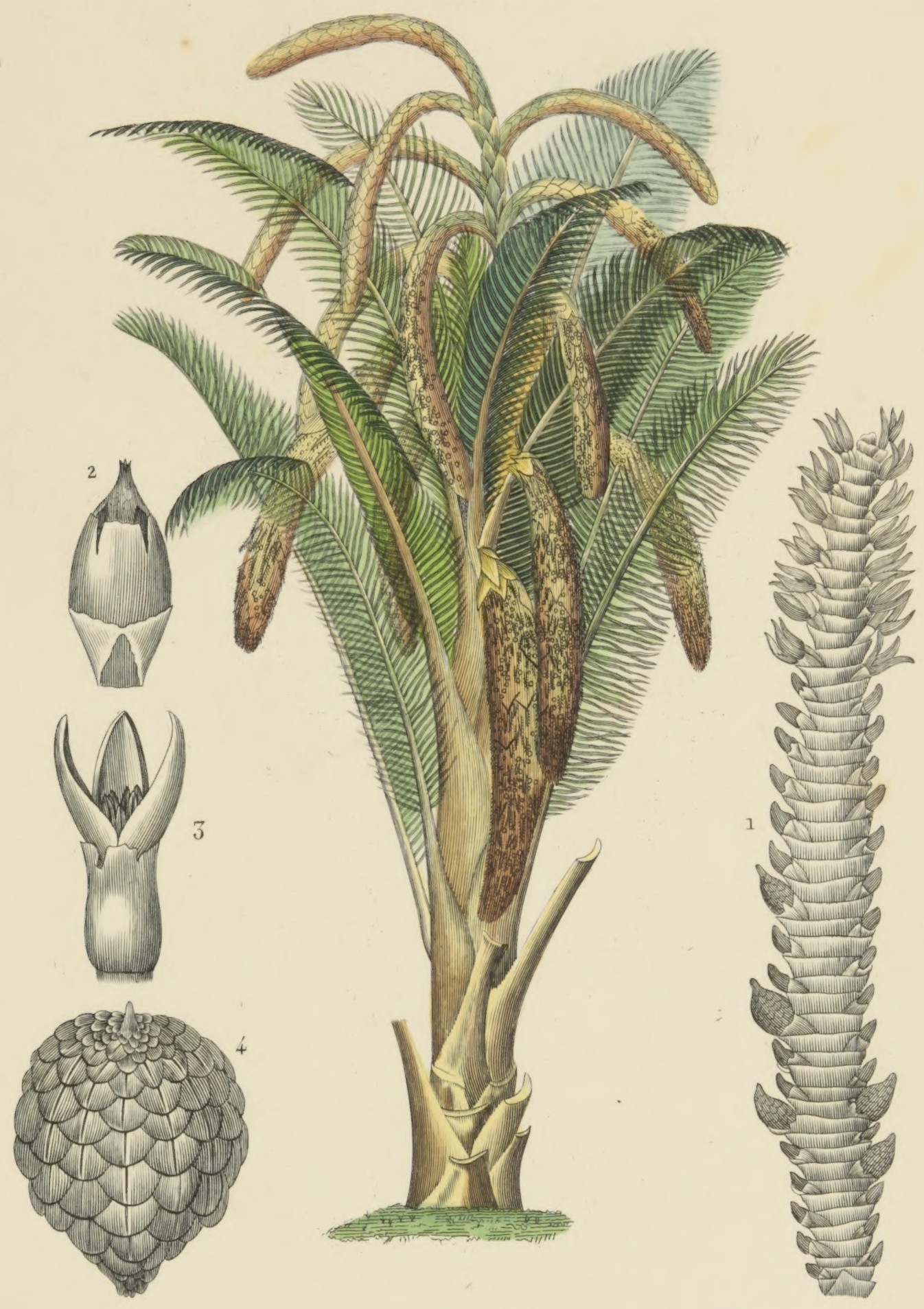

SA A

SAGUS FARTNIFERA.

1.Portion d'un régime deFleurs.grnat. 2. Fleur fertile. 3.fleur stérile.4.Fruit. 


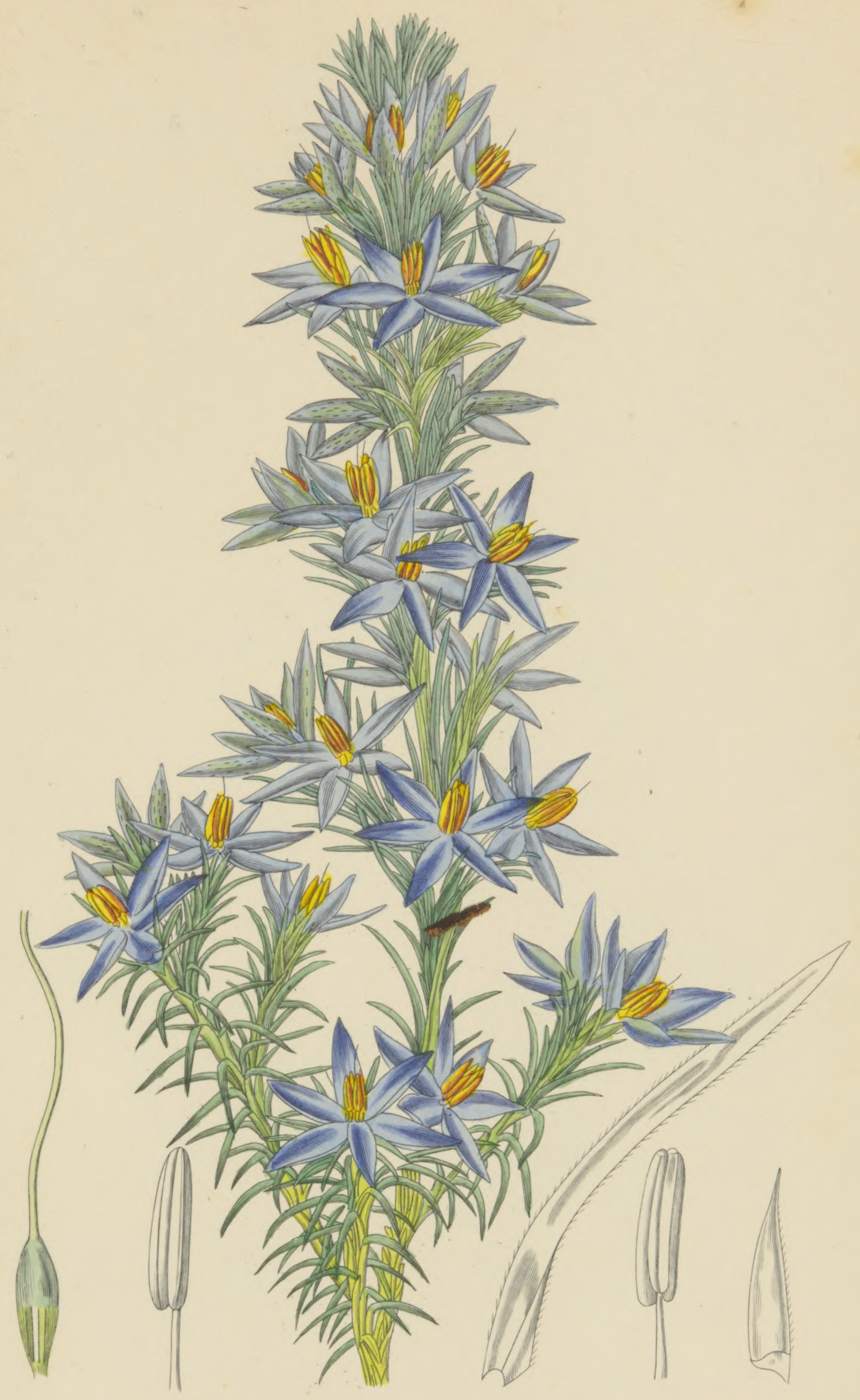

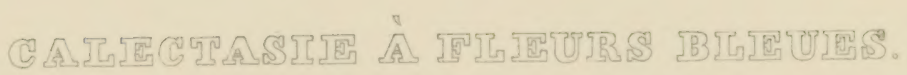

CALECTASTA CYANFA, 